University of Louisville

ThinkIR: The University of Louisville's Institutional Repository

Electronic Theses and Dissertations

$8-2010$

\title{
Nyctalopin is required for localization of the TRPM1 channel to the retinal depolarizing bipolar cell dendrites.
}

Jillian Nydam Pearring

University of Louisville

Follow this and additional works at: https://ir.library.louisville.edu/etd

\section{Recommended Citation}

Pearring, Jillian Nydam, "Nyctalopin is required for localization of the TRPM1 channel to the retinal depolarizing bipolar cell dendrites." (2010). Electronic Theses and Dissertations. Paper 1107. https://doi.org/10.18297/etd/1107

This Doctoral Dissertation is brought to you for free and open access by ThinkIR: The University of Louisville's Institutional Repository. It has been accepted for inclusion in Electronic Theses and Dissertations by an authorized administrator of ThinkIR: The University of Louisville's Institutional Repository. This title appears here courtesy of the author, who has retained all other copyrights. For more information, please contact thinkir@louisville.edu. 
NYCTALOPIN IS REQUIRED FOR LOCALIZATION OF THE TRPM1 CHANNEL TO THE RETINAL DEPOLARIZING BIPOLAR CELL DENDRITES

By

Jillian Nydam Pearring

B.A. Boston University, 2004

M.S. University of Louisville, 2007

\author{
A Dissertation \\ Submitted to the Faculty of the \\ Graduate School of the University of Louisville \\ in Partial Fulfillment of the Requirements \\ for the Degree of
}

Doctor of Philosophy

Department of Biochemistry and Molecular Biology

University of Louisville

Louisville, KY

August 2010 
COPYRIGHT 2010 by Jillian Nydam Pearring

All rights reserved 



\title{
NYCTALOPIN IS REQUIRED FOR LOCALIZATION OF THE TRPM1 CHANNEL TO THE RETINAL
} DEPOLARIZING BIPOLAR CELL DENDRITES

\author{
By \\ Jillian Nydam Pearring \\ B.A. Boston University, 2004 \\ M.S. University of Louisville, 2007
}

A Dissertation Approved on

AUGUST 9, 2010

By the-following Dissertation Committee:

Ronald G Gregg, PhD - Chair

William L Dean, PhD

Richard C Feldhoff, PhD

Maureen A McCall, PhD

Brian Wat PhD 


\section{DEDICATION}

To my beloved husband Jason Reed Willer 


\section{ACKNOWLEDGEMENTS}

I would like to thank my professor, Dr. Ronald Gregg, for all of his patience and mentorship. He helped me to critically think through experimental problems, confidently defend my research, develop my ability to write a scientifically and was always there for questions and guidance. I would also like to thank my committee members, Dr. Maureen McCall, Dr Brian Wattenberg, Dr. Richard Feldhoff and Dr. William Dean, for their support and suggestions. They helped to guide my progress and provided input on experiments and data analysis. In addition, their labs were always open to borrow reagents and ask for technical support; especially the Wattenberg lab that taught me how to culture cells and provided me space in their hood for two years. I would also like to thank the members of the McCall lab who joined our lab for weekly journal clubs. These meetings developed my ability to critically analyze scientific papers and understand electrophysiological techniques and analysis. I would also like to thank my collaborators, Dr. Nawy and Dr. Peachey, for their mentorship and interest in my success. They provided positive criticism with an outside perspective that helped me acquire a broad scientific outlook. I am also blessed with a loving family and friends who supported me throughout this process. 


\section{ABSTRACT \\ NYCTALOPIN IS REQUIRED FOR LOCALIZATION OF THE TRPM1 CHANNEL TO THE RETINAL DEPOLARIZING BIPOLAR CELL DENDRITES}

\section{Jillian N Pearring}

August 2, 2010

Mutations in the NYX gene, encoding the novel protein nyctalopin, cause human congenital stationary night blindness type 1 (CSNB1). CSNB1 is an X-linked recessive condition and is identified by a loss of the electroretinogram (ERG) b-wave while the a-wave remains intact (Miyake et al., 1986). The b-wave in normal patients is generated by the depolarization of one class of bipolar cells, which receive signals from the photoreceptors. This indicates that CSNB1 is a loss of signaling from the photoreceptors to the depolarizing bipolar cells. Normal stimulation of photoreceptors by light causes the photoreceptors to hyperpolarize and reduce the amount of neurotransmitter, glutamate, released into the synaptic cleft. The decrease in glutamate causes Grm6, a metabotropic glutamate receptor, at the postsynaptic depolarizing bipolar cell dendrites to reduce its activity. This signal is relayed by a second messenger system to result in the opening of a non-selective cation channel, which depolarizes one class of bipolar cells. The signaling to the non-selective cation channel in depolarizing bipolar cells is disrupted when nyctalopin is absent. Recent data shows that the non-selective cation channel is the transient receptor potential subfamily M member 1 (TRPM1) channel (Shen et al., 2009; Morgans et al., 2009; Koike et al., 2009). The focus of my studies has been to understand the function of nyctalopin in the depolarizing bipolar cells. I characterized TRPM1 expression in the retina, investigated the interdependence between nyctalopin, Grm6 and TRPM1 and determined the interactions between nyctalopin and Grm6 or TRPM1. There are five retinal splice variants of TRPM1. Variants containing all six transmembrane domains have a developmental expression profile similar to bipolar cells. Immunohistochemical analysis shows TRPM1 is localized to BC somas and dendritic tips where it co-localizes with YFP-nyctalopin. Nyctalopin interacts with the TRPM1 channel is required for proper localization of the TRPM1 channel to the bipolar cell dendritic tips. 
TABLE OF CONTENTS

PAGE

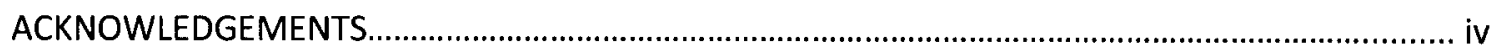

ABSTRACT

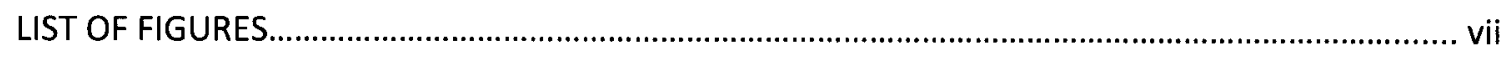

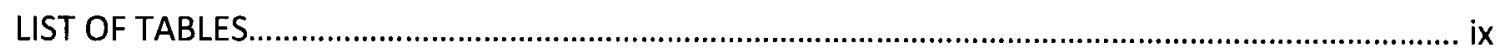

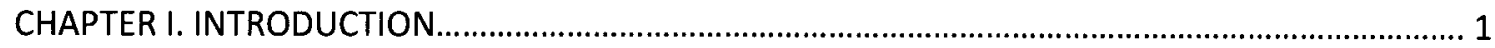

CHAPTER II. DETERMINE THE EXPRESSION PATTERN OF TRPM1 IN THE RETINA.......................... 35

CHAPTER III. DETERMINE THE INTERDEPENDENCE OF NYCTALOPIN, TRPM1 AND GRM6

EXPRESSION IN DEPOLARIZING BIPOLAR CELLS IN THE MURINE RETINA .......................61

CHAPTER IV. DETERMINE INTERACTIONS BETWEEN NYCTALOPIN, GRM6 OR TRPM1................. 82

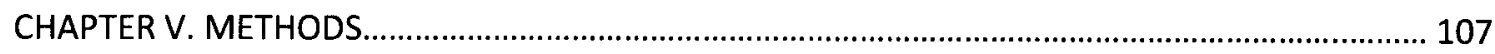

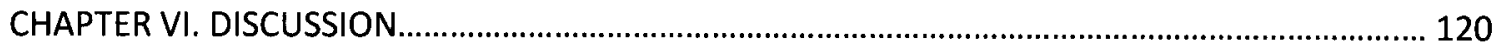

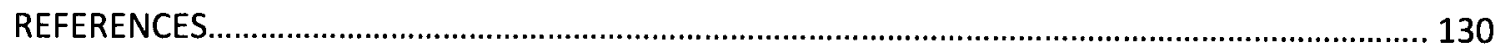

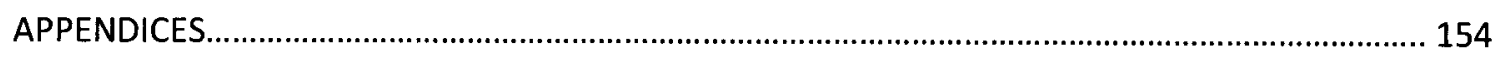

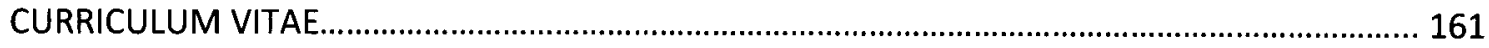




\section{LIST OF FIGURES}

\section{FIGURES}

PAGE

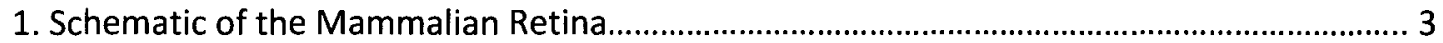

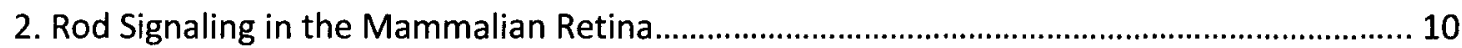

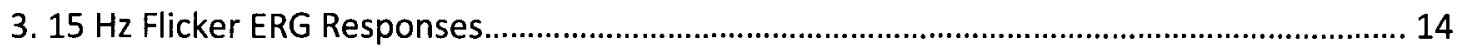

4. Grm6 Signal Cascade in Depolarizing Bipolar Cells.................................................................. 23

5. Nyctalopin Structure, Function and Expression in the Retina............................................... 26

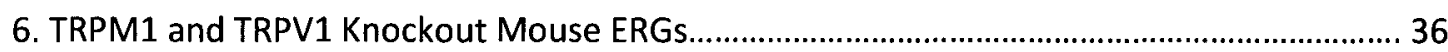

7. TRPM1 Splice Variants from Retina CDNA

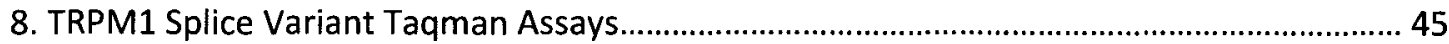

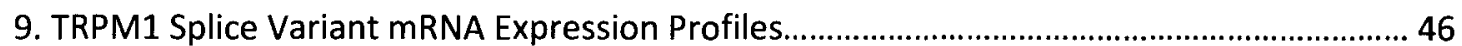

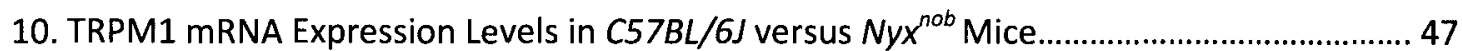

11. Immunohistochemical Markers for the Retinal OPL........................................................... 49

12. Immunohistochemical Analysis of TRPM1 Antibodies in $C 57 B / / 6 J$ Retina............................50

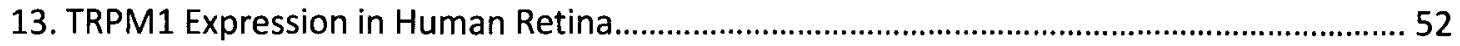

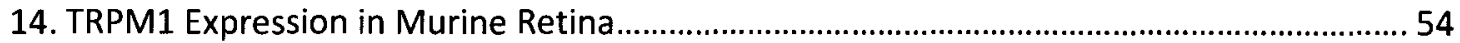

15. The TRPM1 Channel Co-localizes with Nyctalopin in DBC Dendrites...................................56

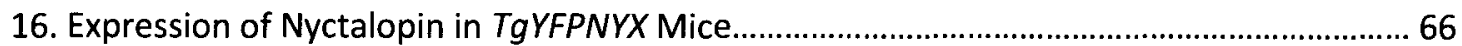



18. Quantitative Analysis of the TRPM1 Fluorescence in $C 57 B / / 6 J$ and $N y x^{n o b}$ Mice............... 69

19. Nyctalopin Expression in the Absence of TRPM1 


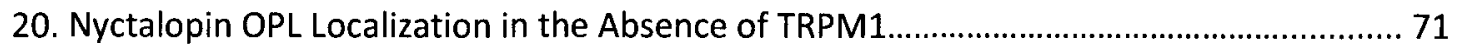



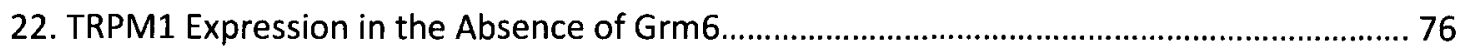

23. Immunohistochemistry of TRPM1 in Grm6 Mutant Mice.................................................. 78

24. Western Blot for YFP-nyctalopin from Retinal Membrane Preparations............................... 87

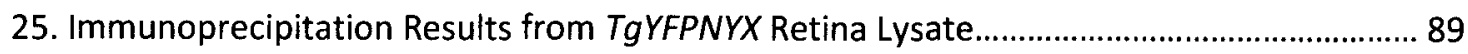

26. Western Blot of Protein Fractions from GFP Immunoprecipitation.....................................90

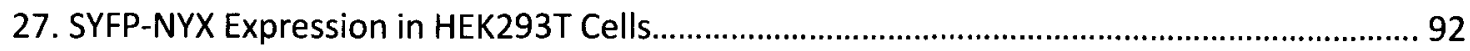

28. Nyctalopin Purification from Transiently Transfected HEK293T Cells.................................... 94

29. Western Blot from In Vitro Binding Study of SYFP-NYX and Grm6

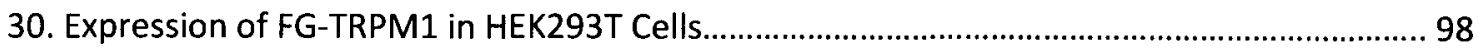

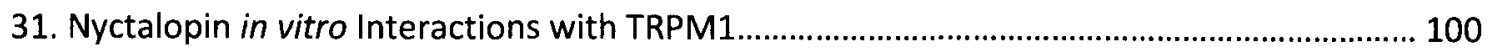

32. Whole Cell Patch Clamp Recordings from HEK293T Cells................................................... 103

33. Summary of Interdependence Experiments of DBC Membrane Proteins............................. 122

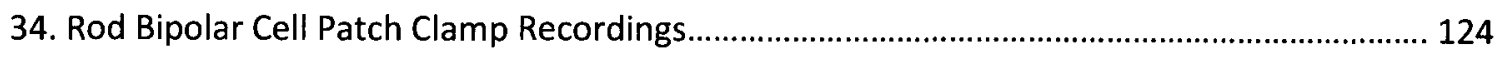

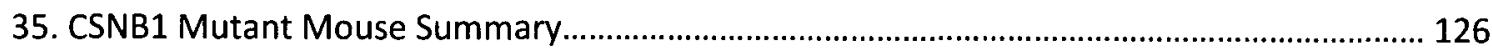

36. Models for nyctalopin and TRPM1 interactions in DBCs................................................ 129

37. Immunohistochemistry of in vivo Electroporated into $\mathrm{Ny} \mathrm{x}^{\text {nob }}$ Mice..................................... 155

38. Normalized ERG from YFP-nyctalopin in vivo Electroporated into Nyx nob Mice.................. 157

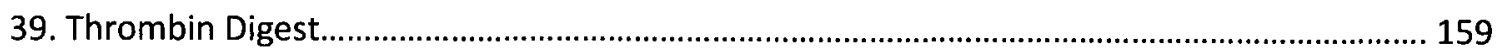




\section{LIST OF TABLES}

TABLES

PAGE

1. PCR Primer Sets for TRPM1 and TRPV1 in Murine Retina..................................................... 40

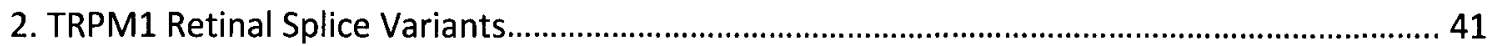

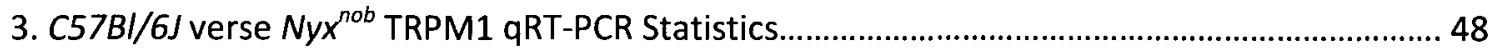

4. Commercial TRPM1 antibodies Screened for Expression in the Human and Murine Retina... 48

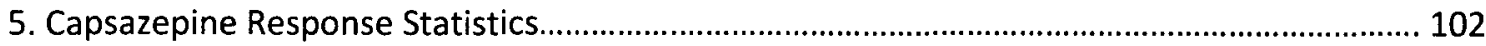

6. qRT-PCR Taqman Primers and Probes for TRPM1 Splice Variants in Murine Retina............... 111

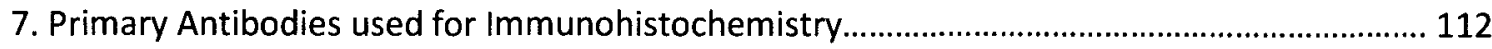

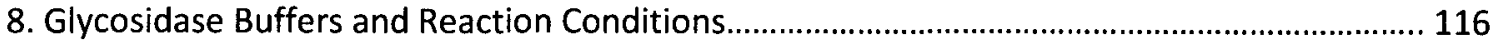

9. Primary Antibodies used for Immunoprecipitation and Western Blot..................................... 117

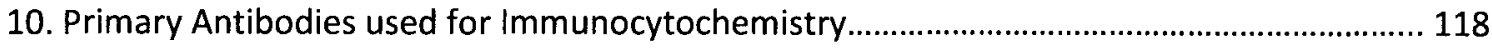

11. Expression of PR and DBC Proteins in the OPL of CSNB1 Mutant Mice................................. 122 


\section{CHAPTER I. INTRODUCTION}

\section{Retina Organization and Function}

The retina is a part of the central nervous system and is designed to processes complex visual signals. One way the retina processes visual information is via multiple types of retinal neurons and their connections (Dowling \& Boycott, 1966; Boycott \& Dowling, 1969; Wässle \& Boycott 1991; MacNeil \& Masland, 1998). In 1892, Santiago Ramon y Cajal published the first anatomical description of retinal cells using a Golgi silver nitrate stained retina (Cajal, 1892; Figure 1).

The retina is divided into three nuclear layers, that contain cell bodies, and two plexiform layers that contain synapses (Cajal, 1892). The outer retina is composed of the photoreceptor outer segments (OS) and inner segments (IS), their somas comprise the outer nuclear layer (ONL) and their axon terminals extend into the outer plexiform layer (OPL). The inner retina is divided into the inner nuclear layer (INL), inner plexiform layer (IPL), ganglion cell layer (GCL) and inner limiting membrane (ILM) (Figure 1). The terminals of the photoreceptors (PRs) form synapses in the OPL with bipolar cells (BC) and horizontal cells ( $\mathrm{HC}$ ). There are two types of PRs, the cones and rods (Figure 1A, B). The INL contains the BCs, HCs and amacrine cells (ACs) (Figure 1B, C, D). BCs send their axons into the IPL to synapse with $\mathrm{AC}$ and/or ganglion cell (RGC) dendrites. The innermost nuclear layer, the $G C L$, contains RGC and displaced $A C$ somas. The RGCs are the output cells of the retina, whose axons form the optic nerve and transmit the visual signal to rest of the brain. In most mammalian retinas, the optic nerve also contains incoming blood vessels that vascularize the retinal layers. In addition, the retina contains radial 
Müller glial cells that span the retina (Figure 1F). Müller cells send out processes that surround every neuronal cell body as well as form the OLM and ILM that serve as a barrier between the retina and the rest of the eye.

The visual signal initiated in the photoreceptors travels through the retina via vertical excitatory pathways, from the PRs to BCs then to RGCs. This pathway employs glutamate as its primary neurotransmitter and is divided into two parallel, ON and OFF pathways. ON and OFF responses are created by the type of glutamate postsynaptic receptors expressed on $\mathrm{BCS}$ dendrites. ON BCs express metabotropic glutamate receptor $6, \mathrm{Grm} 6$, and depolarize to light increments. These will be referred to as depolarizing bipolar cells (DBCs). OFF BCs express ionotropic glutamate receptors, AMPA/Kainate, and depolarize in response to light decrements. These will be referred to as hyperpolarizing bipolar cells (HBCs). The ON and OFF pathways are conserved at the next synapse in the retina where BCs synapse with ACs and RGCs. The IPL is divided into two functionally discrete sublaminae. This functional segregation is mirrored by a structured difference in the IPL. In sublaminae A, the most distal part of the IPL, OFF BCs axon terminals form synapses with OFF RGC dendrites (Figure 1G). In sublaminae B, the proximal part of the IPL, ON BCs axon terminals form synapses with ON RGC dendrites (Figure $1 \mathrm{H}$ ). RGCs that stratify in both sublaminae they are called bi-stratified (Figure 11). 


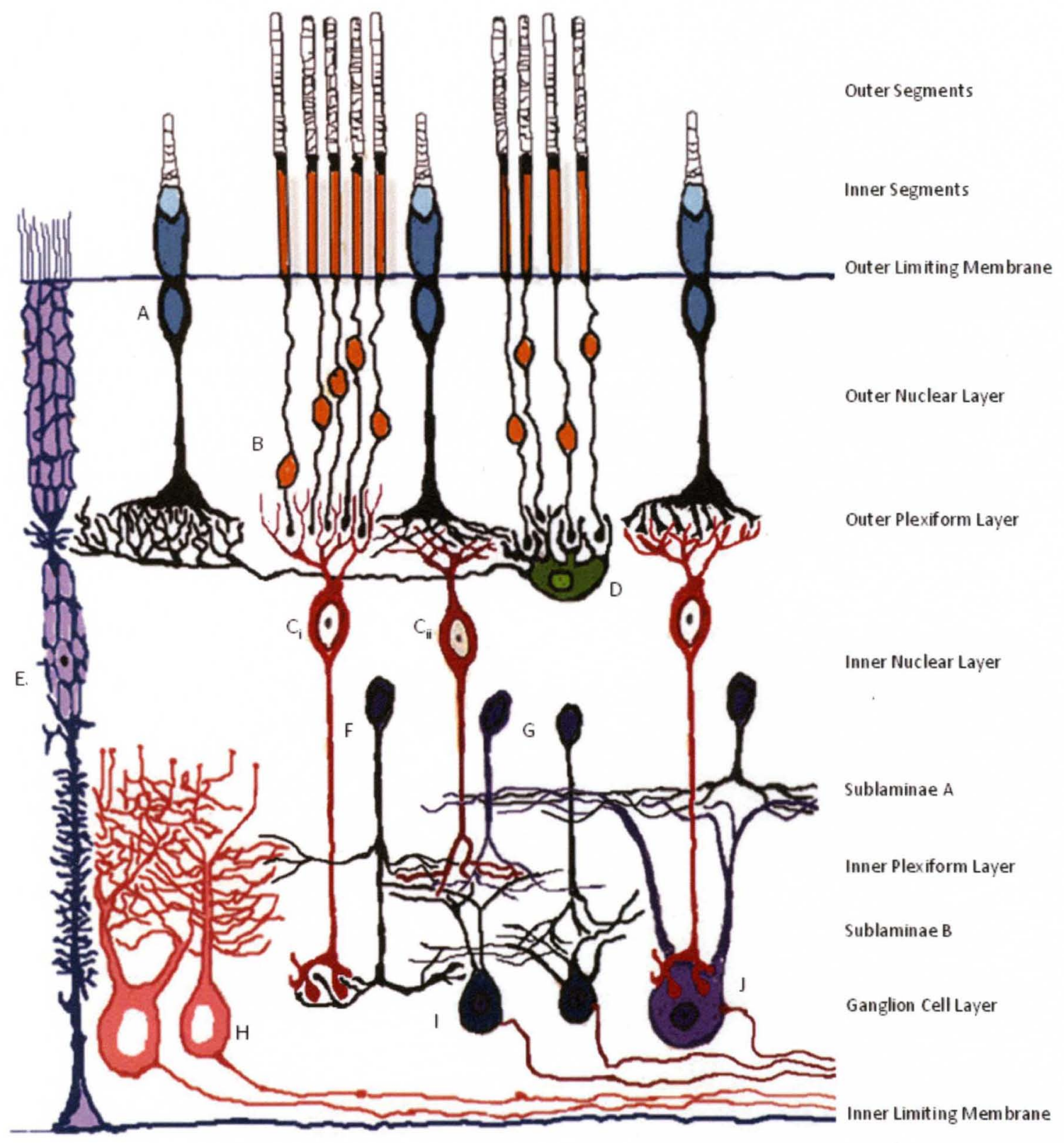

Figure 1: Schematic of the mammalian retina. $(A)$ Cone Photoreceptor $(B)$ Rod Photoreceptor $\left(C_{i}\right)$ Rod Bipolar Cell ( $C_{i i}$ ) Cone Bipolar Cell (D) Horizontal Cell (E) Muller Glial Cells (F) All Amacrine Cell (G) Amacrine Cell $(\mathrm{H})$ Bi-stratified RGC (I) ON-center RGC (J) OFF-center RGC. The laminated nomenclature is depicted on the right side of the figure. Modified from "Structure of the Mammalian Retina" c.1900 By Santiago Ramon y Cajal. 
Each synapse in this vertical pathway is modulated by lateral inhibitory pathways. In the outer retina, the inhibitory neurons are HCs (Mills \& Massey, 1994) and are electrically coupled to each other and provide negative feedback to the PRs and negative feed forward to BCs (Dowling, 1970; Baylor et al., 1971; Mangel, 1991; Vigh \& Witkovsky, 1999). Glutamate release from PRs binds AMPA/Kainate receptors on HCs to elicit a depolarization (Lasater \& Dowling, 1982; Tachibana, 1985). Whether the HC employs a neurotransmitter or utilizes hemi-channel gap junctions to feedback onto PRs or feed forward to BCs remains unclear. The inner retina contains ACs that form inhibitory synapses with BC axon terminals and RGC dendrites. In mammals, there are as many as 25 types of ACs, which are classified based on their dendritic morphology, stratification in the IPL, and the neurotransmitter they use, primarily GABA or Glycine.

Converging vertical and lateral inputs generate receptive fields (RFs), which are characterized by classic center/surround organization. Center/surround organization is fundamental to encoding contrast between images and their background and spatial activity. In the OPL, PR input is believed to form the center response of the $B C$ (Werblin \& Dowling, 1969). If this signal was directly transmitted to the RGCs, without information from the surround, it would be blurry. However, the electrically coupled HCs with wide receptive fields add an inhibitory surround component to the $\mathrm{BC}$ signal. The surround response could reflect the negative feedback pathway from HCs to PRs and/or direct inhibitory input from the HCs to BCs. Importantly, the $\mathrm{HC}$ contribution is thought to depict the background intensity and help to fine tune the $\mathrm{BC}$ response.

Visual processing begins with the conversion of light into an electrical signal in the PRs. PRs consist of an outer segment filled with stacks of membranes containing a visual pigment, opsin, an inner segment containing a connection cilium, mitochondria and endoplasmic 
reticulum, a cell body containing the nucleus and a synaptic terminal where neurotransmission to second order neurons occurs. Humans have three types of cones, which are responsible for color vision and operate under normal daylight levels. There is only one type of rod PRs that functions in dim light. Cone PRs are distinguished by the type of opsin they express. L-cones are known to be maximally sensitive to wavelengths peaking at $564 \mathrm{~nm}$ (red), M-cones at $533 \mathrm{~nm}$ (green) and S-cones at $437 \mathrm{~nm}$ (blue) (Gouras, 1984). Rod PRs contain only one type of visual pigment, called rhodopsin, with peak sensitivity at $500 \mathrm{~nm}$ (blue-green).

In the primate as well as some mammals, rod and cone photoreceptors are differentially distributed across the retina. In the fovea cone PRs are concentrated at maximum density, with exclusion of the rods (Yamada, 1969; Curcio et al., 1987). The fovea is responsible for high acuity central vision. In the parafoveal region, the rods break up the dense hexagonal packing of the cones but still maintain an organized architecture with evenly spaced cones surrounded by rings of rods. The peripheral retina is rod-dominated, with exclusion of cones.

Phototransduction begins when light stimulates a retinal chromophore, which is derived from vitamin A and covalently bound to the transmembrane protein rhodopsin (Koutalos \& Ebrey, 1986). Rhodopsin is a G-protein coupled receptor that is linked to the heterotrimeric Gprotein, transducin. Transducin contains an $\alpha-, \beta$-, and $\psi$-subunit. Upon absorption of a photon of light, the retinal chromophore isomerizes from the 11-cis form to an all-trans form that changes the conformation of rhodopsin to metarhodopsin II, the active form of rhodopsin. Metarhodopsin II exchanges GDP for GTP on transducin. The active GTP bound form of transducin causes the $G \alpha_{t}$ subunit to dissociate from the $G \beta \gamma$ subunits. The $G \alpha_{t}$ subunit then activates a cGMP-phosphodiesterase (PDE). PDE is a heterotetramer consisting for two catalytic subunits ( $\alpha \beta$ in rod, $\alpha \alpha$ in cones) and two identical regulatory $\gamma$ subunits (Beavo, 1995). Activation of PDE occurs by binding of $G a_{t}-G T P$ to the PDEY subunit, which induces a structural 
change and activation of the catalytic subunit. Activated PDE then hydrolyzes CGMP to 5'-GMP, reducing cytoplasmic levels of CGMP (Dizhoor, 2000). The decrease in cGMP results in closure of a cGMP-gated cation (CNG) channel, preventing influx of sodium that results in PR hyperpolarization (Haynes et al., 1986). The hyperpolarization results in closure of an L-type voltage-dependent calcium channels (L-VDCCS) at the PR axon terminals. As the local calcium concentration in the axon terminals decreases, the glutamate released by the PR into the synaptic cleft also decreases.

The VDCCs in PRS are regulated by graded changes in membrane potential, which transmits information over a wide range of light intensities. One way this dynamic range is achieved is by using a unique chemical synapse at the PR terminal called the synaptic ribbon (Dowling, 1987). The ribbon is part of a specialized type of presynaptic active zone. It is composed of electron-dense processes that extends from the site of release into the cytoplasm of the axon terminal and is covered with hundreds of synaptic vesicles (Rao-Mirotznik et al., 1995; von Gersdorff, 2001). The ribbon synapse is found in the CNS at synapses where there is continuous release of neurotransmitter. It is thought to be designed to sustain high, long-lasting rates of neurotransmitter release that are modulated by small changes in membrane potential (DeVries \& Baylor, 1993; Rieke \& Schwartz, 1996; von Gersdorff et al., 1996; Parsons \& Sterling, 2003; Heidelberger et al., 2002). In the retina, this specialized synapse is also found in the second order neurons, the BCs (von Gersdorff et al., 1996).

Two types of bipolar cells, the DBC and HBC, monitor the change in synaptic glutamate released by PRs and are named based on their response to a light increment (Nawy \& Jahr, 1991; Masu et al., 1995; Saito \& Kaneko, 1983; Attwell et al., 1987). DBCs express metabotropic glutamate receptors, Grm6, at their dendrites (Nakajima et al., 1993). The DBC synapse is signinverting, so light induced hyperpolarization of PRs decreases glutamate release and causes DBC 
depolarization and creates an excitatory response correlated with light onset. HBCs express ionotropic glutamate receptors of the AMPA/Kainate family at their dendrites (Gilbertson et al., 1991; DeVries \& Schwartz, 1999; DeVries, 2000). The HBC synapse is sign-conserving, so light induced hyperpolarization of PRs leads to decreased glutamate release and causes HBC hyperpolarization. At light offset, the increased glutamate concentration binds to AMPA/Kainate receptors and depolarizes the $\mathrm{HBCs}$ and creates an excitatory response correlated with light offset. The opposing DBC and HBC responses establish the ON and OFF parallel pathways, respectively in the retina.

There are 8-12 types of cone BCs in the vertebrate retina and 6-8 are DBCs (Boycott \& Wässle, 1991; Euler \& Wässle, 1995; Ghosh et al., 2004). There is only a single morphological type of rod DBC (Dacheux \& Raviola, 1986). DBC dendrites make invaginating synapses with cone and rod PRs (Kolb, 1970). In the dark, glutamate concentration in the OPL is high and the ligand binding site of the Grm6 receptor is predominately occupied. This keeps a G-protein cascade active, a non-specific cation channel closed, and the DBC hyperpolarized (Slaughter \& Miller, 1981). Recently the non-selective cation channel in the DBCs was identified as the transient receptor potential subfamily M member 1 (TRPM1) (Bellone et al., 2008; Shen et al., 2009; Morgans et al., 2009; Koike et al., 2009). When illumination increases, PRs hyperpolarize decreasing glutamate release, which in turn decreases the activity of the DBC Grm6 receptor and its G-protein cascade opening the TRPM1 channel. Influx of calcium and sodium through TRPM1 depolarizes the DBC.

In addition to invaginating synapses, $\mathrm{HBC}$ also form basal junctions with cone PRs. When glutamate binds AMPA/Kainate receptors on HBCs, they open directly to cause depolarization. This response is transient because the AMPA/Kainate receptor rapidly moves into a desensitized, poorly conducting state (Jonas et al., 1993; Paternain et al., 1998). Therefore, in 
prolonged darkness when synaptic glutamate remains high the AMPA/Kainate receptors on HBCs are desensitized. When light increases, synaptic glutamate decreases and the AMPA/Kainate receptors recover from desensitization. This system enables the HBCs to signal decrements in illumination, but respond poorly to an increase in light intensity.

The ON and OFF parallel pathways developed in the cone BCs are transmitted to ACs and RGCs in the IPL. The synapse between BCs and RGCs in the IPL is sign conserving so the primary signal transmitted to the RGC is determined by the type of $\mathrm{BC}$ providing the input (Raviola \& Raviola, 1982). The RGC response is made up of the light evoked activity from the excitatory parallel pathways shaped by the integrative inhibitory networks. RGC responses are sent to the brain via action potentials that propagate down axons that form the optic nerve, optic chiasm, and optic tract.

\section{Rod Pathways}

The human visual system has the ability to detect a single quantum of light (Hecht et al., 1942). This high level of performance is accomplished using the rod PRs, which transmit signals to the inner retina by three known pathways (Figure 2). The primary rod pathway is used under the dimmest light conditions and utilizes rod DBCs (Field \& Rieke, 2002). In this pathway rod DBCs do not synapse directly onto RGCs, but instead the excitatory signal is transmitted via a narrow-field bistratified AC, the All (Kolb, 1977; Strettoi et al., 1990, 1992). All ACs form electrical synapses with cone DBCs and inhibitory synapses with HBCs (Pourcho \& Goebel, 1985). Release of glutamate from rod BCs activates AMPA/Kainate receptors on the All ACs causing depolarization (Dacheux \& Raviola, 1986). The All AC depolarization is transferred through the sign conserving gap junction causing the cone DBCs to depolarize. The depolarization also causes the All AC to release glycine that inhibits HBCs (Figure 2, Sassoè-Pognetto et al., 1994, Wässle et al., 2009). All ACs receive input from many rod DBCs that convergence before synaptic 
output to RGCs. This provides pooling and amplification of the rod signal so our visual system is very sensitive.

The secondary rod pathway uses electrical coupling between rod and cone PRs (Deans et al., 2002; Zhang \& Wu, 2005). The rod signal is then transmitted through the cone pathway (Figure 2, Nelson, 1977). A single rod spherule contains between three and five gap junctions, which originate from either M- or L-cones (Ahnelt et al., 1990). Rod-cone coupling functions under mesopic conditions when rod DBC signals begin to saturate, so are transmitted to the cones via rod-cone gap junctions (Smith et al., 1986). This pathway is faster than the primary rod pathway (Sharpe et al., 1989; Stockman et al., 1995).

The tertiary rod pathway involves synaptic connections between rods and a subset of HBCs (Figure 2, West, 1978; Hack et al., 1999; Tsukamoto et al., 2001). When light increases, rods hyperpolarize causing a subset of HBCs to hyperpolarize due to decreased glutamate bound to the AMPA/Kainate receptors. When light decreases and rods depolarize, the HBCs then depolarize. Therefore, the synapse between a rod and the HBCs can respond to light-off in a mammalian retina. Though the function of this pathway remains unclear, it was recently shown to be the fastest rod pathway and respond to decreased illumination (Li et al., 2010). The presence of multiple rod pathways optimize signaling under a broad range of low light intensities and expands the range of rod mediated vision. 


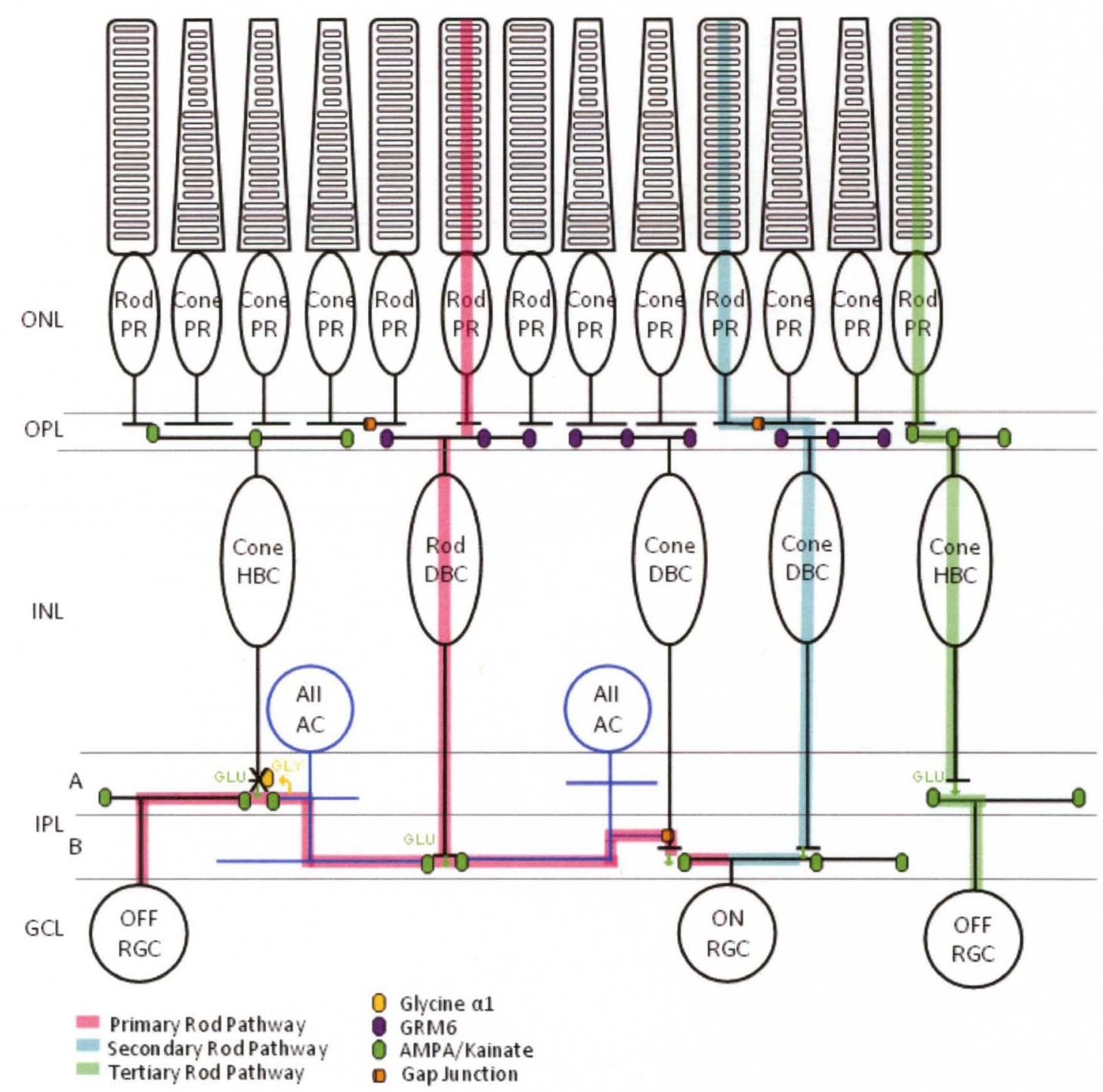

Figure 2: Rod signaling in the mammalian retina travels via three routes. The primary rod pathway (pink, rod $\rightarrow D B C \rightarrow A l l A C \rightarrow H B C$ ). During light onset rods stop releasing glutamate onto rod $D B C$, which provide an excitatory input to All amacrine cells, which stimulate ON RGC through cone DBCs and inhibit OFF RGCs through HBCs. Signaling in this pathway depends on the Grm6 signaling at the DBC. The secondary rod pathway (blue, rod $\rightarrow$ cone $\rightarrow B C$ ). The electrical coupling between rods and cones sends the rod signals to into the cones, signaling both cone DBCs and HBCs and their respective RGCs. The tertiary rod pathway (green, rod $\rightarrow$ HBCs) provides a direct glutamatergic input via AMPA/Kainate receptors to a subset of cone HBCs and consequently OFF RGCs. 


\section{Congenital Stationary Night Blindness}

Congenital stationary night blindness (CSNB) is a group of genetic retinal disorders that cause profound loss of rod-mediated function without evidence of retinal degeneration (Ripps, 1982). Individuals with CSNB can be diagnosed using an electroretinogram (ERG). The ERG is a non-invasive, gross potential recording from the corneal surface that reflects light evoked retinal activity. A flash ERG records the gross potential from the retina to a single flash of light. Upon a flash of light the retina first responds with a cornea-negative ERG a-wave, reflecting the lightinduced closure of CNG channels along the PR outer segment membrane (Lamb, 1996; Robson \& Frishman, 1998; Xu et al., 2003). This is followed by a cornea-positive ERG b-wave, reflecting the subsequent depolarization of the BCs (Robson \& Frishman, 1998; Sharma et al., 2005). The flash ERG can be used to discriminate the two major forms of CSNB; Schubert-Bornschein and Riggs. Schubert and Bornschein (1952) described the ERG phenotype of one form of CSNB in which there was a normal ERG a-wave, while the ERG b-wave amplitude was greatly decreased. The normal amplitude and kinetics of the ERG a-wave with abnormal ERG b-wave suggests that while the PR response is normal there is a failure of synaptic transmission to the DBCs. The second type of CSNB was described by Riggs (1954) in which both the ERG a-wave and b-wave amplitudes were drastically reduced suggesting abnormalities in the PR signal transduction cascade.

X-linked, autosomal recessive and dominant pedigrees of Schubert-Bornschein CSNB have been identified (Carr et al., 1966; Miyake et al., 1986; Kabanarou et al., 2004; Abramowicz et al., 2005). The X-linked form of the Schubert-Bornschein type CSNB has been divided into two separate types, complete and incomplete (Miyake et al., 1986). The complete form of CSNB is characterized by no recordable ERG b-wave, while patients with incomplete CSNB have a recordable ERG b-wave although it is subnormal in amplitude. The complete and incomplete 
forms are now referred to as CSNB1 and CSNB2, respectively. Molecular cloning has identified the underlying genes in both X-linked CSNB1 and CSNB2. CSNB1 is caused by mutations in the NYX gene encoding the novel protein nyctalopin (Bech-Hansen et al., 2000; Pusch et al., 2000). In humans, more than 30 mutations have been mapped in the NYX gene (Bech-Hansen et al., 2000; Pusch et al., 2000; Jacobi et al., 2002; Zeitz et al., 2005b). CSNB2 is caused by mutations in the CACNA1F gene, encoding an $\alpha 1$ subunit of voltage-dependent Ca1.4 channel (L-VDCC) (BechHansen et al., 1998; Strom et al., 1998). More than 40 mutations have been identified in CACNA1F pedigrees (Bech-Hansen et al., 1998; Strom et al., 1998; Wutz et al., 2002; Jacobi et al., 2003; Zeitz et al., 2005b).

Autosomal recessive Schubert-Bornschein CSNB (arCSNB) have also been classified into the complete (arCSNB1) and incomplete (arCSNB2) forms depending on the severity of the ERG b-wave reduction. Patients with arCSNB1 have no recordable ERG b-wave, where as individuals with arCSNB2 have a very small ERG b-wave. arCSNB1 is caused by mutations in the GRM6 gene encoding the metabotropic glutamate receptor 6 (Grm6) (Dryja et al., 2005; Zeitz et al., 2005a) and in the TRPM1 gene encoding the transient receptor potential channel subfamily M1 (TRPM1) (Li et al., 2009; van Genderen et al., 2009; Audo et al., 2009; Nakamura et al., 2010). Both genes are integral members of the DBC transduction cascade. Individuals with arCSNB2 have mutations in the CABP4 gene encoding the calcium binding protein 4. CABP4 is localized in the PR synaptic terminals and modulates calcium influx through the L-VDCC (Zeitz et al., 2006).

As the mutations underlying the CSNB1 and CSNB2 were identified, it became clear that where the genes are located within the PR-BC synapse is important in understanding the phenotype. CSNB1 involves mutations in genes expressed post-synaptically, in the DBCS dendrites that are essential to the transduction cascade. CSNB2 is caused by mutations in genes expressed pre-synaptically, in the PR terminals that control glutamate release. 
Autosomal dominant mutations of CSNB (adCSNB) in human patients have a no ERG awave phenotype. Mutations have been identified in the RHO gene encoding rhodopsin, the GNAT gene encoding rod transducin, and the PDE6B gene encoding rod cGMP phosphodiesterase beta-subunit (Dryja et al., 1993; Rao et al., 1994; Gal et al., 1994; Dryja et al., 1996; al Jandal et al., 1999; Zeitz et al., 2008). Many mutations in RHO lead to retinitis pigmentosa (RP), which initially resembles CSNB but gradually leads to death of rods in the periphery of the retina. However, four mutations in the RHO gene cause non-progressive adCSNB. These mutations are believed to cause constitutive activation of rhodopsin and its downstream target, transducin, which causes a loss of the ERG a-wave and consequently a loss of the b-wave (Dryja et al., 1993; Rao et al., 1994; al Jandal et al., 1999; Zeitz et al., 2008).

CSNB1 individuals with mutations in the different genes expressed in the DBCs can be further distinguished using a $15-\mathrm{Hz}$ flicker ERG, which discriminate the slow and fast rod pathways. At low scotopic luminance levels, the $15-\mathrm{Hz}$ flicker is dominated by the slow, sensitive rod pathway (rod $\rightarrow$ rod DBC $\rightarrow$ All AC) and at high scotopic levels by the faster (rod $\rightarrow$ cone $\rightarrow$ HBC) pathway. These rod pathways are separated by a delay of approximately $33 \mathrm{~ms}$, or half of the $15-\mathrm{Hz}$ period. When they are used equally by the retina, at about $-0.56 \log s \operatorname{sot} t d \cdot s$, there is destructive interference between the two signals causing a null ERG (Sharpe et al., 1989; Sharpe et al., 1993). The 15- $\mathrm{Hz}$ flicker ERG in normal individuals starts with a measurable amplitude at low light. The ERG amplitude decreases as luminance increases, reaches a null at about $-0.56 \mathrm{log}$ $s \operatorname{sot} t d \cdot s$, before increasing in amplitude at higher luminances (Figure $3 \mathrm{~A}$ ).

Patients with X-linked CSNB1 have no slow rod signals and the faster rod signals have reduced amplitudes, which is consistent with an altered ON pathway (Scholl et al., 2001; Figure 3C). The $15-\mathrm{Hz}$ ERG amplitude is reduced at light levels below $-0.56 \log s \cot t d \cdot s$, but is normal at light levels above $-0.56 \log s c o t \mathrm{td} \cdot \mathrm{s}$. This $15-\mathrm{Hz}$ phenotype was also identified in arCSNB 
patients with TRPM1 mutations (Figure 3D, van Genderen et al., 2009). In contrast, arCSNB

patients with mutations in GRM6 have a complicated profile with normal responses at low and

high scotopic levels and very large responses in between these light levels (Figure 3B, Zeitz et al.,

2005a; van Genderen et al., 2009). This distinctive GRM6 ERG phenotype is suggested to be due

to the interaction between the secondary and the tertiary rod pathway (rod $\rightarrow$ OFF-HBCs). The

15- $\mathrm{Hz}$ flicker ERG can be used to distinguish patients with mutations in GRM6 from Nyx or

TRPM1 (Zeitz et al., 2005a; van Genderen et al., 2009).
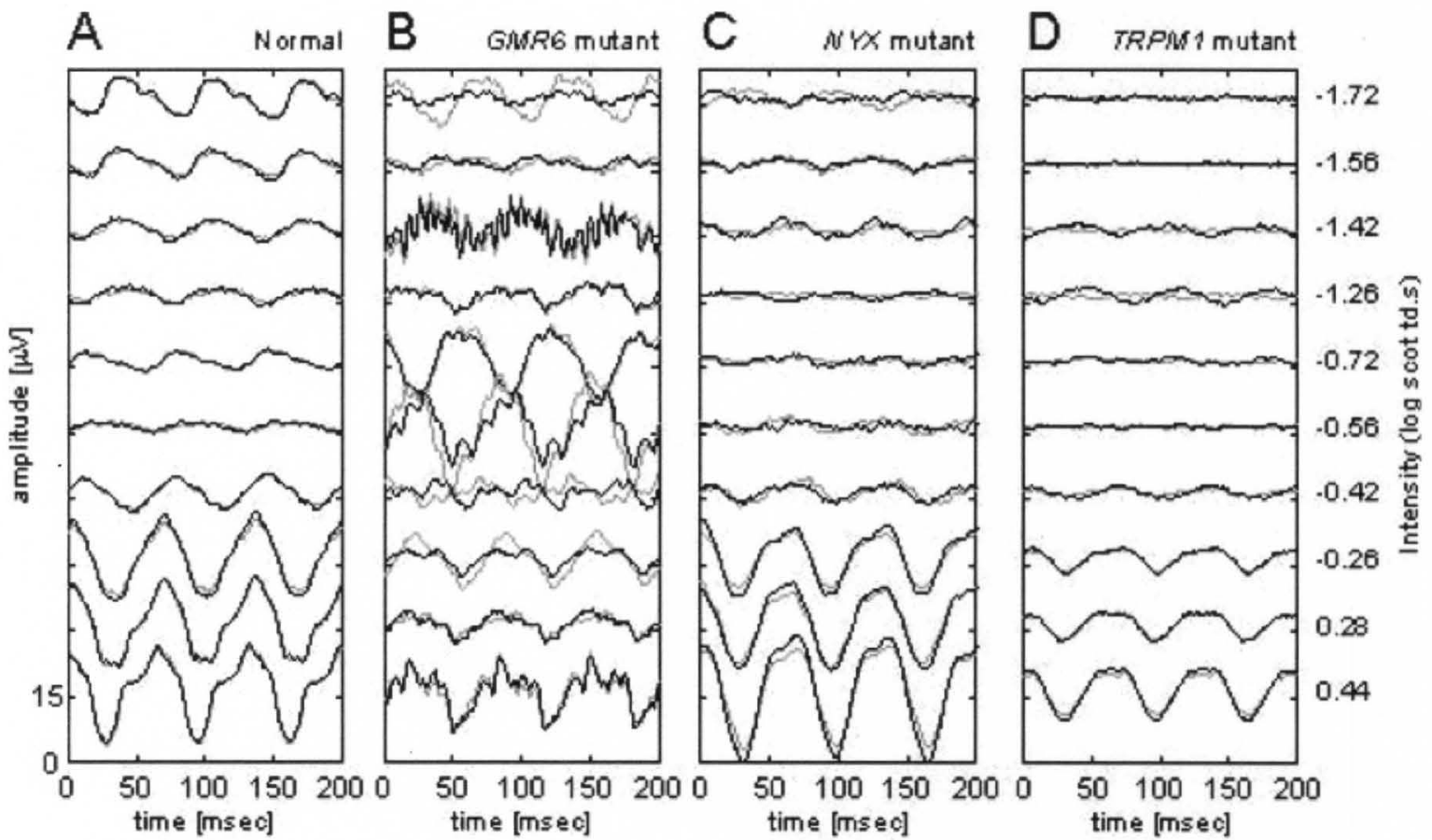

Figure 3: $15 \mathrm{~Hz}$ flicker ERG responses from representative (A) normal healthy subjects and probands with (B) GRM6, (C) NYX and (D) TRPM1 mutations. ERG responses are from the right eye (Grey) and the left eye (Black). Scotopic $15 \mathrm{~Hz}$ flicker ERG responses to a series of 11 stimuli of increasing intensities starting at $-1.72 \log s c o t$ td.s (top trace) to $0.44 \log s c o t$ td.s (bottom trace). Between -1.72 and $-0.56 \log$ scot td.s rod signals in the normal retina uses the primary (rod $\rightarrow$ ON bipolar $\rightarrow$ All amacrine cell) pathway. At mesopic levels ( $-0.56 \log \operatorname{scot} t d \cdot s$ and up) the secondary (rod $\rightarrow$ cone $\rightarrow$ bipolar) pathway is used. At approximately $-0.56 \log s \operatorname{sot} t d \cdot s$, the signals transferred through the primary and secondary pathways are equal in amplitude, but lag one another by half a phase, causing destructive interference and a null response, see normal subject (A). In probands with NYX (C) or TRPM1 (D) mutations, ERG responses from the primary rod pathway are not present, where as responses from the secondary rod pathway are within normal limits, causing reduced amplitudes. In the proband with GRM6 mutation (B), ERG responses could be measured at all intensities, but the responses had abnormal phase behavior. Modified from van Genderen et al., 2009. 


\section{Mouse Models of CSNB}

In the past decade, several mouse models for CSNB have been identified or generated and these mutations provide both functional and molecular comparisons to the human disease. Mouse-based studies have provided insights into the underlying mechanism of the CSNB group of retinal disorders and are generally classified into two main groups: pre-synaptic mutations (CSNB2) and post-synaptic mutations (CSNB1).

\section{CSNB2 Mouse Models}

Mouse models of CSNB2, like the human counterparts, have mutations in genes expressed on PR terminals. These mutations disrupt glutamate release to the second order neurons. Glutamate from PR terminals is initiated by calcium influx through L-VDCC. Cone PRs express L-VDCCs that are comprised of $\alpha 1 F$ and $\alpha 1 D$ subunits, while rod PRs contain L-VDCCs with only the a1F subunit (Morgans et al., 2001; Morgans et al., 2005).

To study the contribution of the L-VDCC $\alpha 1 \mathrm{~F}$ subunit to PR signal transmission a CACNA1F knockout mouse, CACNA1 $F^{\text {tm1Tbh }}$, was generated (Mansergh et al., 2005). In addition, a spontaneous mouse mutant was identified, $C A C N A 1 F^{\text {nob2 }}$ (Chang et al., 2006). Retina morphology in the two CACNA1F mutant mice is very similar. The synapses between PRs and BCs in both mutants are disrupted, resulting in an abnormally thin OPL. In addition, dendrites of both the DBCs and HCs extend into and across the ONL (Mansergh et al., 2005; Chang et al., 2006). Functionally, both CACNA1F mutant mice display a normal ERG a-wave with significantly reduced ERG b-wave at both light and dark adapted conditions (Mansergh et al., 2005; Chang et al., 2006). However, though the ERG phenotype is similar, the underlying visual responses were drastically different. The $C A C N A 1 F^{t m 1 T b h}$ mouse has no visually evoked responses in the superior colliculus, implying absence of activity through RGCs (Mansergh et al., 2005). The RGCs in CACNA1F $F^{\text {nob2 }}$ mice, however, retain near normal RF organization and sensitivity to light under 
photopic conditions and have relatively normal visual performance, as measured by the optokinetic response, when compared to wildtype (Chang et al., 2006; Doering et al., 2008).

The conflicting phenotypes between the two CACNA1F mutant mice led to further analysis of their gene expression by Doering and colleagues (2008). The CACNA1 ${ }^{\text {nob2 }}$ mice contain a transposable element (Mus musculus transposon ETn) inserted in exon 2 of the CACNA1F gene. Analysis of the CACNA1F mRNA identified alternative splicing in which the ETn element was removed in approximately $10 \%$ of the transcripts (Doering et al., 2008). This alternately spliced transcript produces a functional L-VDCC protein that differs from wild type LVDCC protein by 22 amino acids at the $\mathrm{N}$-terminal (Doering et al., 2008). The altered $\mathrm{N}$-terminus in CACNA1F ${ }^{\text {nob2 }}$ cannot interact with filamin (Doering et al., 2008). Filamin is a protein that binds the actin cytoskeleton and participates in anchoring membrane proteins, such as L-VDCCs. This suggests that the mutant L-VDCC in CACNA1F $F^{n o b 2}$ mice might not be properly anchored in the PR terminals. The additional residues at the $\mathrm{N}$-terminus in the $C A C N A 1 F^{\text {nob2 }}$ mice did not alter the function of the L-VDCC, which could explain the relatively normal visual responses measured in CACNA1F $F^{\text {nob2 }}$ mice.

Mice with the targeted deletion of the $\alpha 1 D$ subunit, which is shown to be expressed in

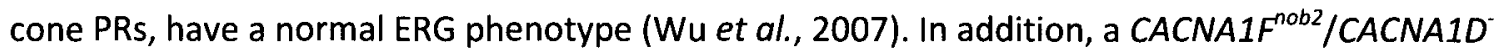
1- double knockout had a similar dark adapted ERG as the CACNA1F nob2 mouse (McCall et al., 2008). This suggests that though the cone terminals express both the $\alpha 1 \mathrm{~F}$ and $\alpha 1 \mathrm{D}$ subunits of $L-$ VDCC the $\alpha 1 D$ subunit is not a major contributor to the ERG $b$-wave. What the $\alpha 1 D$ subunit of the L-VDCC is doing in the cone PR terminals remains to be determined.

Mutations in five other genes involved in release of neurotransmitter from PRs have been reported to cause decreased ERG b-waves. These pre-synaptic mutations disrupt calcium signaling and prevent neurotransmitter release, which is consistent with the reduced b-wave 
phenotypes. Mutations in two auxiliary subunits to the L-VDCC, $\beta 2$ and $\alpha 2 \delta 4$, result in abnormal or absent ribbon synapses in rod PRs and thinning of the OPL (Ball et al., 2002; Ruether et al., 2000; Wycisk et al., 2006a). Mutations in bassoon, a component of the ribbon synapse (Dick et al., 2003), CABP4, which encodes calcium binding protein 4 (Haeseleer et al., 2004) and dystrophin, a component of the extracellular matrix (Pillers et al., 1999), all have reduced ERG $b$ waves and abnormal $\mathrm{BC}$ and $\mathrm{HC}$ dendritic morphology.

The functional and morphological abnormalities in the CSNB2 mutants all produce alterations in calcium entry or glutamate release. In addition, these mutants have disrupted OPL architecture, which indicates the underlying genes may be involved in synapse formation between PR and BCs. Calcium influx can activate intracellular pathways directly at the synapse or mediate changes in nuclear gene transcription (Rosen et al., 1994; Ghosh \& Greenberg, 1995; Curtis \& Finkbeiner, 1999). These intracellular processes have the potential to alter cell surface molecules that may be critical to normal synapse formation. In vitro studies have described links between L-VDCCs and PR development. Blocking the influx of ions through the VDCCs inhibited axon outgrowth, varicosity formation and vesicle protein synthesis in developing rods (Zhang \& Townes-Anderson, 2002; Nachman-Clewner et al., 1999). These results suggest that the presence or activity of L-VDCCS is required for normal development and synapse formation in the outer retina. Defects in calcium entry may explain the altered synaptic architecture of the OPL in pre-synaptic mutant mice. However, glutamate release may also be a key component guiding $B C$ and $H C$ neurites to synapse with PR terminals. Glutamate has been shown to play a role in synaptic motility (Engert \& Bonhoeffer, 1999; Maletic-Savatic et al., 1999; Wong et al., 2000; Wong \& Wong, 2001). In the IPL, glutamate release from BCs regulates the formation of synapses between BCs and RGCs (Kerschensteiner et al., 2009). Though the mechanism remains 
unknown, pre-synaptic signals either regulate synaptogenesis or help maintain synaptic structures.

\section{CSNB1 Mouse Models}

Mutations in seven genes expressed by the DBCs have helped identify proteins critical to DBC signal transduction. In some cases, mice were identified by their ERG no b-wave phenotype and positional cloning subsequently identified mutations in the underlying genes ( $\mathrm{Ny} x^{\text {nob }}$, Pardue et al., 2003; Grm6 $6^{\text {nob3 }}$, Maddox et al., 2008; Grm6 $6^{\text {nob4 }}$, Pinto et al., 2007). Other mice were produced by gene targeting of genes known to be expressed in BCs (Grm6 ${ }^{\text {tm1Nak }}$, Masu et al., 1995; Gnao1 ${ }^{\text {tm1Lib }}$, Dhingra et al., 2000; Gnb5 ${ }^{\text {tmck }}$, Rao et al., 2007; TRPM1\% ${ }^{\%}$ Koike et al., 2009) or identified in knockout mice generated by Lexicon Genetics (TRPM1 ${ }^{\text {tm1lex }}$, Shen et al., 2009). There are three alleles that disrupt the Grm6 gene; Grm6 $6^{\text {Tm1Nak }}$ (Masu et al., 1995); Grm6 $6^{\text {nob3 }}$ (Maddox et al., 2008); Grm6 ${ }^{\text {nob4 }}$ (Pinto et al., 2007), two alleles that disrupt G-proteins in the Grm6 cascade, $\mathrm{Ga}_{\alpha}$ and $\mathrm{G} \beta 5$ (Gnao1 ${ }^{\text {tm1Lbi }}$ and $G n b 5^{\text {tmck }}$ ) (Dhingra et al., 2000; Dhingra et al., 2002; Rao et al., 2007), one allele that disrupts nyctalopin, $N y x^{\text {nob }}$ (Pardue et al., 1998, Gregg et al., 2003) and two alleles that disrupt the TRPM1 gene, TRPM1 $1^{\text {Imlex }}$ and TRPM1 ${ }^{\%}$ (Shen et al., 2009; Koike et al., 2009). With the exception of $G n b 5^{t m c k}$ mice whose OPL thickness is abnormal, the gross morphology of the retina in these mutants is normal (Tagawa et al., 1999; Pinto et al., 2007; Dhingra et al., 2000; Pardue et al., 1998; Morgans et al., 2009). The decrease in OPL thickness could be explained by the existence of two Gnb5 retinal splice variants. A short form of Gnb5 (Gnb5-S) is expressed in the BCs and a long form of Gnb5 (Gnb5-L) is expressed in PRs. Thus, the pre-synaptic mutation of the Gnb5-L isoform would be consistent with other presynaptic mutations with altered OPL morphology. To determine the postsynaptic contribution of Gnb5-S alone a Gnb5- $L$ transgene was introduced into the rods of $G n b 5^{\text {tmck }}$ mice (Rao et al., 2007). Transgenic expression of Gnb5-L failed to rescue the b-wave or the OPL defects 
suggesting Gnb5-S is involved in OPL synaptic development (Rao et al., 2007). The synaptic connections between PRs and BCs are formed between P10 to P14 (Takada et al., 2004). Gnb5-S is present during this critical period, so could be playing a developmental role (Rao et al., 2007). Immunohistochemical and electron microscopic studies of $N y x^{\text {nob }}$ and Gnao $1^{\text {tm1lbi }}$ mice show that the OPL architecture is normal (Pardue et al., 2001; Dhingra et al., 2000). However, electron microscopic studies of $G r m 6^{T m I N a k}$ mice found a subset of DBC that contained ectopic ribbon synapses in their dendrites (Ishii et al., 2009). In Grm6-deficient mice the ectopic dendritic ribbons might be due to alteration in DBC activity that increases intracellular trafficking of synaptic proteins resulting in an overload and mislocalization of synaptic proteins (Ishii et al., 2009).

The functional impact of the deficits in DBC signaling on RGC function has been reported for several CSNB1 mutants. RGC responses of $N y x^{\text {nob }}$ and $\mathrm{Grm} 6$ mutant mice are the best characterized and indicate that mutations in the same cascade can produce drastically different outcomes on downstream cellular function. In Grm6 mutants the number of ON-responsive RGCs is reduced, while the OFF-center RGCs are relatively normal compared to wildtype mice. When an ON RGC response was elicited the response was delayed and the firing rate reduced compared to wildtype (Pinto et al., 2007; Renteria et al., 2006; Maddox et al., 2008). These delayed ON responses are initiated by the OFF pathway (Renteria et al,. 2006). Taken together these results indicate that the absence of signaling through the Grm6 receptor results in an abnormal ON pathway. Interestingly, $\mathrm{Ny} x^{\text {nob }}$ mice lack RF organization in both ON and OFF RGCS (Demas et al., 2006, Gregg et al., 2007; Heflin et al., 2010). This suggests that the Grm6 mutation is restricted to the ON pathway, where as the lack of nyctalopin expression causes global RGC abnormalities. This variation may result from differences in the state of the TRPM1 channel, which will affect the DBC resting state and subsequent downstream visual processing. Patch 
clamp recordings from DBCs in $N y x^{n o b}$ mice lack holding currents compared to an average inward holding current of 40pA in wildtype DBCs (Gregg et al., 2007). Further, variance analysis and determination of open probability suggests that the TRPM1 channel is absent or closed in Nyx ${ }^{\text {nob }}$ DBCs (Gregg et al., 2007). Supporting this conclusion are studies of RGC response properties from TRPM $1^{\text {tm1lex }}$ mice, which exhibit a RGC phenotype similar to Nyx ${ }^{\text {nob }}$ mice (Heflin et al., 2010). While the status of the TRPM1 channel in the Grm6 mutants is unknown, the difference in RGC phenotypes in Ny $x^{\text {nob }}$ and TRPM1 ${ }^{\text {tm1lex }}$ verse Grm6 mutants suggests the state of the DBCs is different.

\section{Grm6 Cascade in Depolarizing Bipolar Cell}

Synaptic transmission from PRs to $B C$ s initiates the ON and OFF signaling pathways in the retina. This divergence is due to the receptor composition expressed on the dendrites of BCs. The OFF pathway is mediated through the HBCs ionotropic AMPA/Kainate glutamate receptors, while the ON pathway is mediated through the DBCs metabotropic glutamate receptor, Grm6 (Nawy \& Jahr, 1990; Shiells \& Falk, 1990; Nakajima et al., 1993; Nomura et al., 1994; Vardi et al 1998, 2000, 2002).

Grm6 is coupled to the heterotrimeric G-proteins $G \alpha_{0}, G \beta 5$ and $G \gamma$, which were originally identified in the cascade by physiology and gene expression profiling of DBCs (Nawy, 1999; Dhingra et al., 2000, 2002; Watson et al., 1996, Nakajima et al., 2009). The TRPM1 channel is the transduction channel in the DBCs and when signaled to open, depolarizes the DBC. The G-protein second messengers are believed to act directly or indirectly to regulate the TRPM1 channel (Snellman et al., 2008). Although aspects of the transduction cascade remain elusive, significant progress has been made in characterizing many of the modulatory proteins.

A schematic representation of the DBC G-protein cascade is depicted in Figure 4. The DBC cascade is broken down into four key steps required for the cell's normal response to 
increases or decreases in glutamate. First, glutamate released into the synaptic cleft binds to Grm6 transforming it from an inactive to an active state (Nawy \& Jahr, 1990; Nakajima et al., 1993). This initiates signal transduction by exchanging a GDP for a GTP on $\mathrm{Ga}_{0}$. Activated $\mathrm{Ga}_{0}-$ GTP is released from the GBY complex. GTP exchange is believed to be critical to initiate the second stage in DBC signaling cascade, closure of the TRPM1 channel. It is unknown whether $\mathrm{Ga}_{\mathrm{o}}$ and/or G $\beta \gamma$ directly act to close the TRPM1 channel or whether they act through another pathway (Snellman et al., 2008). When luminance increases, glutamate decreases and Grm6 inactivates. Inactive Grm6 stops the exchange of GDP for GTP on $G \alpha_{o}$ and the intrinsic GTPase activity of $\mathrm{Ga}_{0}$ hydrolyzes GTP to GDP. Inactivation of the Grm6 cascade leads to opening of the TRPM1 and the DBC depolarizes. The molecular mechanism regulating this process remains enigmatic. The cascade is initiated again when the luminance decreases and initiates the Grm6 cascade to close TRPM1 and hyperpolarize the DBC until the next light-evoked response. Each step of the DBC transduction cascade and the proteins regulating the kinetics of the response are discussed in more detail below.

Grm6 is a G-protein coupled receptor (GPCRs) that is predicted to have seven transmembrane spanning $\alpha$-helices (Filipek et al., 2003) and a large extracellular ligand binding domain that binds glutamate (O'Hara et al., 1993; Kunishima et al., 2000). The glutamate signal is transmitted across the membrane to a heterotrimeric $\mathrm{G}$ protein coupled on the cytoplasmic surface. The activation and inactivation of Grm6 has been modeled after the crystal structure of rhodopsin (Palczewski et al., 2000; Park et al., 2008). The transmembrane domains of GPCRs are held in the basal state by non-covalent interactions between their side chains (Schoneberg et al., 1995; Kobilka et al., 1998). Upon agonist binding intramolecular interactions are altered to favor an active conformation (Balmforth et al., 1997; Groblewski et al., 1997). Agonist binding is a complex process involving kinetically distinct stages resulting in activation, exchange of GDP 
for GTP on $G \alpha_{0}$, and release of the heterotrimeric G-protein (Swaminath et al., 2004, 2005; Liapakis et al., 2004, Yao et al., 2006; Weis \& Kobilka, 2008).

Glutamate binding activates Grm6, which propagates the signal by exchanging GDP for GTP on $\mathrm{Ga}_{0}$. The GTP exchange is facilitated by a retina variant of Purkinje cell protein-2 (RetPCP2), which was identified as a guanine exchange factor (GEF) in DBCs (Xu et al., 2008). RetPCP2 contains a GoLoco motif that interacts with $G \alpha_{o}$ and accelerates the exchange of GTP for GDP (Luo \& Denker, 1999; Zhang et al., 2002; Xu et al., 2008). Interestingly, Ret-PCP2 facilitates TRPM1 closure in DBCs not by directly acting on the channel, but by altering the G-protein kinetics.

The termination phase of the Grm6 cascade is also assisted by a regulator of G-protein signaling (RGS) protein. Though $\mathrm{G} \alpha_{0}$ has intrinsic GTPase activity, its hydrolysis is facilitated by a RGS protein. RGS11 and RGS7, members of the R7 family of RGCs proteins, are expressed in DBCs (Song et al., 2007; Morgans et al., 2007; Rao et al., 2007; Mojumder et al., 2009) and accelerate GTP hydrolysis of $\mathrm{G} \alpha_{0}$ (Hooks \& Harben, 2004). Recently, RGS7 and RGS11 knockout mice were shown to have a delayed ERG b-wave, which reflect slower inactivation of the Gproteins and subsequent opening of TRPM1 in DBCs (Mojumder et al., 2009; Chen et al., 2009; Zhang et al., 2010). Interestingly, in RGS7/RGS11 double knockout mice the b-wave delay was even greater suggesting a parallel involvement for RGS7 and RGS11 in regulating the shut off phase of the DBC response (Mojumder et al., 2009; Chen et al., 2009; Zhang et al., 2010). The time course of the ERG b-wave in RGS7/RGS11 knockout mice is still faster than intrinsic GTP turnover on $\mathrm{Ga}_{0}$, so it is likely that another protein is serving as the RGS for $\mathrm{Ga}_{\circ}$ (Bourne \& Stryer, 1992; Mojumder et al., 2009; Chen et al., 2009; Zhang et al., 2010).

Termination of the G-protein signaling cascade results in the depolarization of the DBC. In 2009, Shen et al identified TRPM1 as the transduction channel responsible for the 
depolarizing light response in DBCs. Identifying the TRPM1 channel as the nonselective cation channel signified a major breakthrough. The key question remaining is how TRPM1 is gated by the DBC signaling cascade.

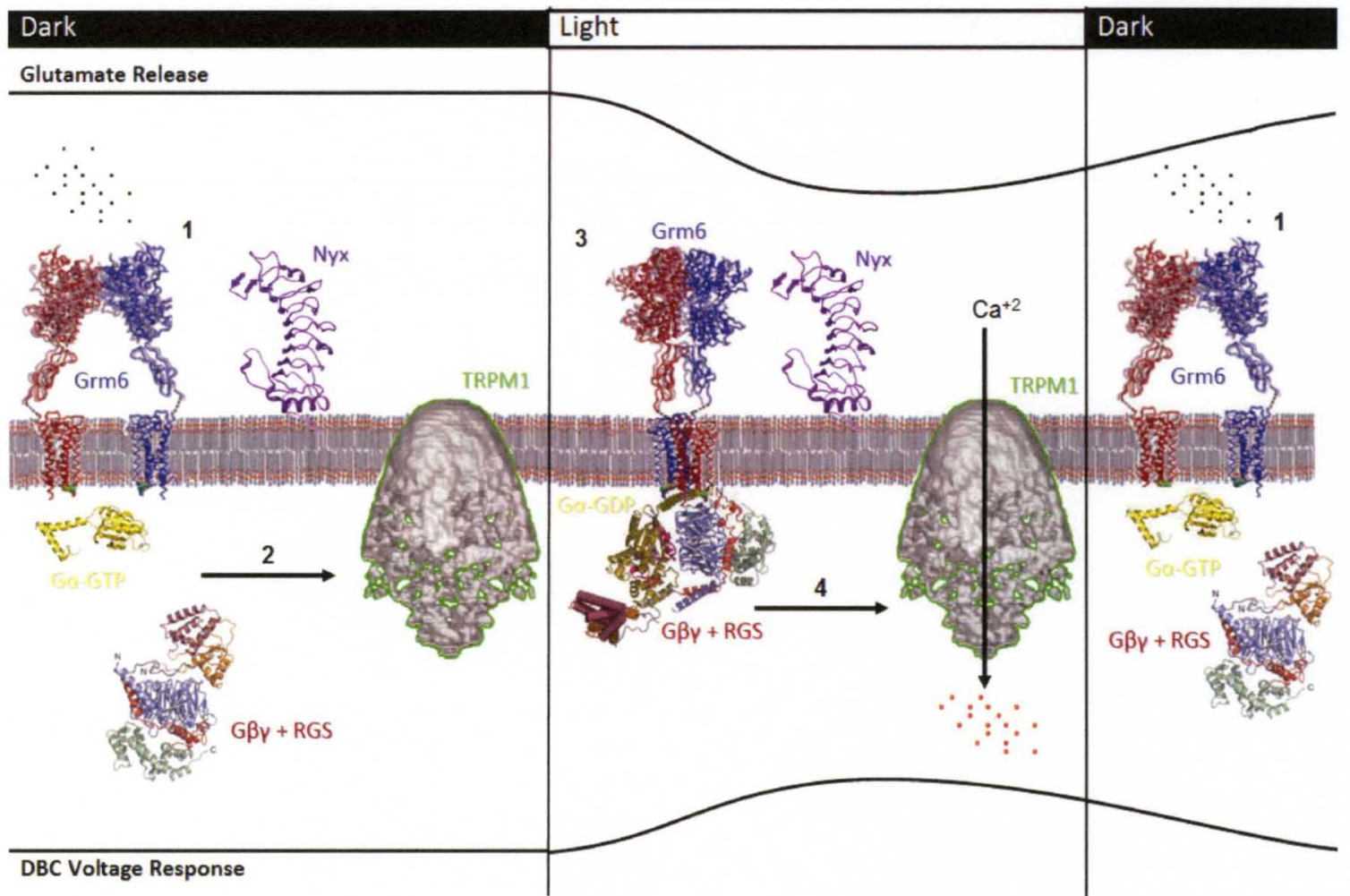

Figure 4: Schematic representation of the Grm6 signaling cascade in depolarizing bipolar cells (DBC). The top trace represents glutamate release over time, and the bottom trace represents voltage response of the DBC. Step 1: In darkness, continuous release of glutamate from PRs activates Grm6 receptors located at the dendritic tips of the DBC. Step 2: Activation of Grm6 leads to an exchange of GDP with GTP on $G \alpha_{0}$, which is facilitated by a GEF, and dissociation of G $\beta \gamma$ from G $\alpha$-GTP. As a result, G $\alpha$-GTP and GBY are in their active forms, either of which could lead to closure of TRPM1 and membrane hyperpolarization. Step 3: When light intensity increases, glutamate release decreases, and GAP activity of $G \alpha_{0}$ hydrolyzes G $\alpha$-GTP to G $\alpha$-GDP. This hydrolysis in DBCs is likely facilitated by an RGS protein. Step 4: G $\alpha$-GDP rebinds $G \beta \gamma$ forming the inactive heterotrimer. Thus, TRPM1 channels are opened and calcium influx depolarizes the membrane. Step 1: When the luminance decreases, glutamate release increases again and Grm6 is activated, reinitiating the cascade. Protein structures were adapted from Cheever et al., 2008; Maruyama et al., 2007; Muto et al., 2007. 


\section{Nyctalopin}

Biochemical and genetic approaches have identified many regulatory proteins of the Grm6 signaling cascade. One such protein, nyctalopin, was identified by positional cloning of the gene responsible for X-linked CSNB1 in humans and mice (Candille et al., 1999; Gregg et al., 2003; Bech-Hansen et al., 2000; Pusch et al., 2000). Nyctalopin is a member of the leucine rich repeat protein (LRR) superfamily (Bech-Hansen et al., 2000; Pusch et al., 2000). These proteins

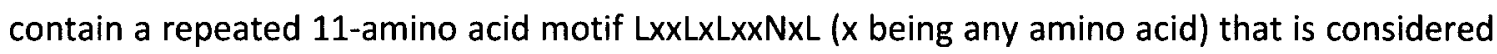
an excellent motif for protein-protein interactions. The first crystal structure of a LRR protein, porcine ribonuclease inhibitor (RNI), helped define the prototypic LRR fold that is characterized by a right-handed solenoid-like morphology (Kobe \& Deisenhofer 1993). Each LRR contributes one $\beta$-strand to the parallel $\beta$-sheet on the inner face of the solenoid. A molecular structure of nyctalopin using the Modbase Modeller System is depicted in Figure 5A. Nyctalopin contains a core 11 leucine-rich repeat region that is flanked by cysteine rich "cap" regions suggesting it is a member of the small leucine-rich repeat proteoglycans (SLRP) (Bech-Hansen et al., 2000; Pusch et al., 2000; Gregg et al., 2003). However, nyctalopin has distinct N-and C-terminal cap motifs. The $\mathrm{N}$-terminal cap contains extra cysteine residues, which are predicted to form an additional disulfide bond. The C-terminal capping motif is not the prototypical ear-repeat as seen in other SLRP proteins, but contains two disulfide bonds that adopt a similar structure as the broader class of LRR proteins. These unique structures have caused speculation that nyctalopin does not belong in the SLRP family (Bella et al., 2006). Another unique feature downstream of the LRR domain is an evolutionarily conserved region with unknown function. In addition, nyctalopin is predicted to be attached to the membrane with the $\mathrm{N}$-terminus extending into the extracellular matrix (Morgans et al., 2005; Zeitz et al., 2005; O'Conner et al., 2005). In humans, nyctalopin is 
attached to the membrane by a GPI anchor, while the mouse protein is predicted to have a single transmembrane domain (Gregg et al., 2003; O'Conner et al., 2005).

The function of nyctalopin in the retina remains enigmatic. In $N y x^{\text {nob }}$ mice, absence of the b-wave in the ERG places the functional deficits in transmission of signals between PRs and DBCs (Pardue et al., 1998). Patch clamp recordings from $\mathrm{Ny} x^{\text {nob }}$ mice show DBCs do not respond to glutamate suggesting nyctalopin is involved in generating the DBC response (Gregg et al., 2007). However, conflicting reports have shown nyctalopin expression in the murine retina at the GCL, DBC and INL, which has caused some debate as to the potential function of nyctalopin (Pesch et al., 2003; Gregg et al., 2003; Morgans et al., 2003). To address this question, Gregg et al (2007) generated a transgenic mouse that expressed an YFP-nyctalopin fusion protein. Immunohistochemistry for the YFP-nyctalopin fusion protein showed robust expression on the dendrites of DBCs in the OPL (Figure 5B). Localization with Grm6 and not Ribeye suggests nyctalopin is postsynaptic (Figure 5B). Most importantly, crossing the TgYFPNYX mouse line with the $N y x^{n o b}$ mice rescued the no b-wave ERG phenotype of the $\mathrm{Nyx}{ }^{n o b}$ mice (Figure $5 \mathrm{C}$ ). Therefore, nyctalopin expression only on DBC dendrites is required for normal function of murine DBCs. However, the mechanism of action of nyctalopin remains to be determined. 
A.

C.
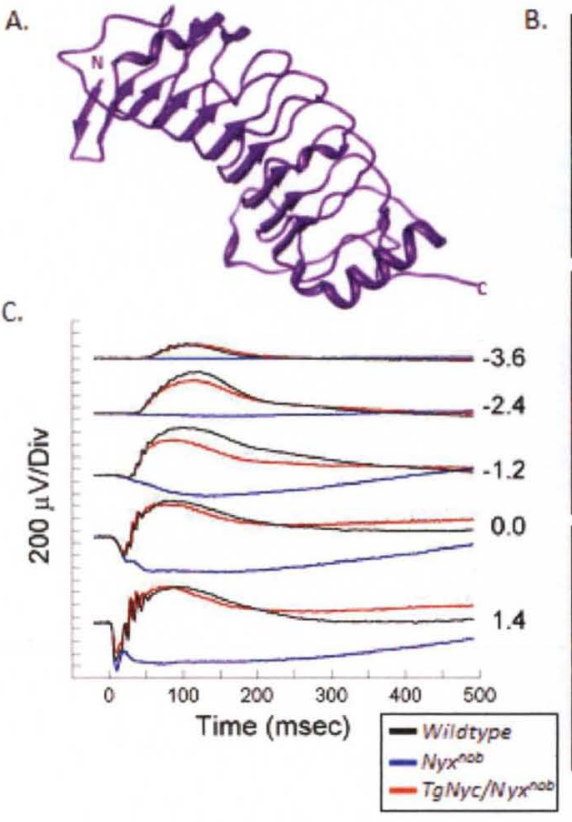

B.
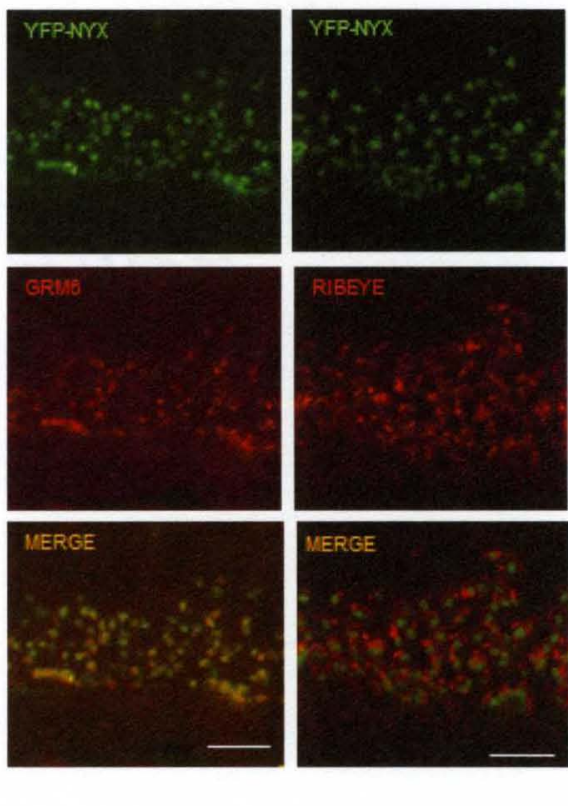

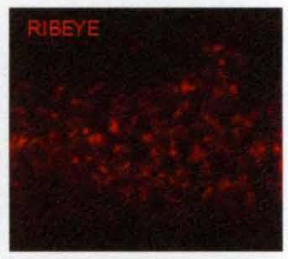

MERGE

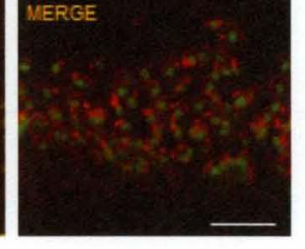

Figure 5: Nyctalopin structure, function and expression in the retina. A. Molecular structure of nyctalopin using the Modbase Modeller System. Nyctalopin is predicted to have a curved structure with $\beta$-sheets (arrows) line the concave side, with helices on the convex side. B. YFP-nyctalopin is localized to the postsynaptic membrane of bipolar dendrites. Staining of TgYFPNyx retinal sections for YFP-nyctalopin (green) and Grm6 (red) or ribeye (red). The merged images show that YFP-nyctalopin is only co-localized with Grm6, but is closely aligned with the presynaptic ribbon maker, ribeye. Scale bar is $5 \mu \mathrm{m}$. C. Transgenic expression of YFP-nyctalopin to BCs restores the ERG b-wave in $\mathrm{Nyx}^{\text {nob }}$ mice. Representative dark-adapted ERGs from control (black traces), $N y x^{\text {nob }}$ (blue traces) and TgYFPNYX 


\section{TRPM1 in Depolarizing Bipolar Cells}

Significant progress had been made in characterizing the properties of the nonselective cation channel in the DBCs. Measurements of the current-voltage relation associated with lightevoked responses showed that glutamate closed a DBC channel with a reversal potential near OmV and had an inwardly rectifying current (Shiells et al., 1981; Nawy \& Copenhagen, 1987; Nawy \& Jahr, 1990). Furthermore, addition of intracellular GTP into DBCs demonstrated that the DBC channel response was mediated by a G protein-coupled mechanism (Nawy \& Jahr, 1990). Key features of the DBC channel are that it is calcium permeable and the influx of calcium triggers a negative-feedback pathway that desensitizes the channel (Sheills \& Falk, 2000; Nawy et al., 2000). The calcium-triggered negative-feedback pathway is activated during PR adaptation to steady illumination when synaptic glutamate levels fall and the number of open DBC channels increases. Calcium entry through the channel closes the DBC channel, which restores the membrane potential and input resistance of DBCs (Shiells \& Falk, 1994; Nawy et al., 2000). This regulation by calcium provides the DBCs with the ability to adapt to changes in ambient light. Though the DBC channel was shown to be potentiated by GTP and depressed by calcium, the channel identity remained elusive until recently.

In 2008, Kim et al screened for retina DBC genes using microarray analysis from a single DBC and identified TRPM1 channel mRNA. In situ hybridization, showed TRPM1 mRNA was expressed in BCs and its developmental expression pattern correlated with other $\mathrm{BC}$ specific genes (Kim et al., 2008). The first report that the TRPM1 channel may be involved in regulating PR to DBC responses came from studies of night blind appaloosa horses. Appaloosa horses carry the leopard complex $(L P)$ gene, which is responsible for distinct coat color patterns (Sandmeyer et al., 2007). Horses homozygous for the LP gene have an ERG consistent with CSNB1 (Sandmeyer et al., 2007). Mapping LP localized the gene to a $6 \mathrm{cM}$ region on equine 
chromosome 1 (ECA1), which contains the TRPM1 gene (Terry et al., 2004). Quantitative RT-PCR analysis of mRNA from the retina of appaloosa horses homozygous for $L P$ had an 1800 fold decrease in TRPM1 expression compared to controls (Bellone et al., 2008). This suggested that TRPM1 is involved in DBC signal transduction. The requirement for TRPM1 in generating the bwave of the ERG was confirmed in TRPM1 knockout mice (Shen et al., 2009). Further studies in mice and non-human primates localized TRPM1 expression to the DBCs (Morgans et al., 2009; Koike et al., 2009; van Genderen et al., 2009). Patch clamp recordings showed the absence of normal light responses in DBCS from TRPM1 $\%$ further suggesting TRPM1 is the DBC cation channel (Koike et al., 2009). When expressed in a heterologous system along with Grm6 and $\mathrm{Ga}_{0}$, TRPM1 activity could be negatively regulated by glutamate (Koike et al., 2009). Collectively, these studies demonstrate that TRPM1 is a required component of the nonselective cation channel critical for DBC function.

The TRPM1 channel is a member of a large family known as transient receptor potential (TRP) channels, which have diverse roles including sensory cell function, thermosensation and cellular redox. There are 7 related families of TRP channels (TRPM, TRPV, TRPC, TRPML, TRPA, TRPN and TRPP) all of which contain a conserved TRP domain. They contain six transmembrane domains and can function as homo- or hetero-tetramers (see review by Venkatachalam \& Montell, 2007). TRPM1 was the initial member of the melastatin TRP family and was identified in a differential display screen analyzing mRNA in malignant verse benign melanocytes (Duncan et al., 1998; Hunter et al., 1998). TRPM1 mRNA is absent in malignant melanoma cells, so has become a prognostic marker for patients with localized melanoma (Duncan et al., 2001). TRPM1 mRNA is present in all stages of embryonal development and is restricted to the skin, brain, ear and eye in adults (Duncan et al., 1998; Takumida et al., 2008; Harteneck et al., 2005). Primary human neonatal epidermal melanocytes have a splice variant for TRPM1, which contains only 
the first 500aa. The short variant does not form an ion channel, but is reported to interact directly with and suppress calcium entry though full-length TRPM1 (Xu et al., 2001). In addition, four $\mathrm{N}$-terminal splice variants of TRPM1 were identified in human melanocytes, brain, and retina (Oancea et al., 2009). The TRPM1 variants were named based on the number of amino acids found at the N-terminal that were different from full length TRPM1, which are 17-TRPM1, 70+TRPM1, 92+TRPM1 and 109+TRPM1. All of the variants are predicted to be ion-channel forming isoforms and two (92+TRPM1 and 109+TRPM1) generated ionic currents similar to endogenous TRPM1 when expressed in human melanoma cells. Interestingly, the five splice variants are differentially expressed in primary and transformed melanocytes, suggesting they may have separate biological functions (Oancea et al., 2009).

The physiology of the TRPM1 channel has been described in melanoma cells, DBCs and heterologous systems. Endogenous TRPM1 currents recorded from mouse melanoma cells display an inward rectifying current with a reversal potential of $0 \mathrm{mV}$ (Oancea et al., 2009), which is consistent with the DBC cation channel currents (Shen et al., 2009). In HEK293T cells, fulllength TRPM1 mediates calcium entry that can be blocked by lanthanum (Xu et al., 2001). Though TRPM1 was localized to the plasma membrane it is predominantly intracellularly localized when expressed in HEK293T cell lines (Xu et al., 2001; Oancea et al., 2009). Additionally, TRPM1 currents in melanoma cells are small, which raises the possibility that their primary function in these cells is intracellular or restricted to specific regions of the plasma membrane. Intracellular vesicles containing TRPM1 could be stimulated to insert more TRPM1 into the plasma membrane in response to particular signals, as shown for TRPM7 and TRPC5 ion channels (Oancea et al., 2006; Bezzerides et al., 2004). Aspects of TRPM1 ion channel trafficking, properties and cellular function remain to be explored. 


\section{Protein Trafficking}

Cells communicate with their surroundings through integral membrane proteins. Expression of membrane proteins on distinct cellular regions is important for cellular function. This is especially evident in neurons, which require very different compositions of membrane receptors and/or channels on their dendrites and axons.

Eukaryotes process membrane proteins through the ER and Golgi apparatus before vesicular translocation to the membrane. Translational targeting of membrane proteins to the eukaryotic endoplasmic reticulum (ER) is mediated by a ribonucleoprotein complex, the signal recognition particle (SRP), and its membrane-associated receptor, the translocon. Secretory or membrane proteins contain a signal sequence, which is recognized by the SRP and used to target the protein to the ER. A typical signal sequence consists of a 9-12 hydrophobic residues at the N-terminus of the protein (Valent et al., 1995; Lee \& Bernstein, 2001; Adams et al., 2002). Proteins that lack a signal sequence can also be transported into the ER by the SRP. They contain a polypeptide with the critical level of hydrophobicity that is flanked by basic residues (Lee et al., 2002; Peterson et al., 2003). For integral membrane proteins any one of their transmembrane domains can serve this function.

SRP-mediated translocation begins as the nascent polypeptide exits the ribosome and the signal sequence is recognized by and binds the SRP, halting translational elongation. The SRP bound to ribosome and nascent polypeptide is transported to the ER membrane. Once at the membrane the SRP forms a complex with the translocon. Following docking with the translocon, the signal sequence is released from the SRP and inserted through the aqueous pore of the translocon into the lumen of the ER. The signal sequence is either cleaved or can remain as a transmembrane region of the protein. After docking of the ribosome onto the translocon, the SRP is released from the translocon in a GTP-dependent manner and free SRP is recycled for 
subsequent rounds of protein targeting (Bourne, 1986; Freymann et al., 1999). When elongation of the peptide resumes, the newly synthesized protein is inserted through the translocon into the lumen of the ER. If a protein contains a highly hydrophobic region, such as an internal transmembrane domain, translational elongation is halted. The hydrophobic transmembrane domain is transferred into the lipid bilayer by a lateral gating mechanism in the translocon where it remains as a single $\alpha$-helical membrane spanning segment. Once inserted into the ER membrane, translational elongation resumes. This stop and transfer mechanism will occur for each transmembrane domain in the polypeptide. Once the entire polypeptide has been translated the ribosome is released from the translocon.

Post-translational glycosylation of proteins is believed to participate in many biological functions such as protein folding, intracellular targeting, immune response, cell adhesion and protease resistance. Glycosylation is initiated in the ER when a precursor oligosaccharide, composed of 14 sugars, is transferred to the amino group on asparagines of newly translated proteins. This action is performed by the membrane-bound enzyme oligosaccharyl transferase. Three glucoses and one mannose are removed from the precursor oligosaccharide while still in the ER. Continued trimming and modifying of the $\mathrm{N}$-linked oligosaccharide occurs in the Golgi apparatus.

The biosynthetic-secretory pathway is involved in the delivery of newly synthesized proteins to the plasma membrane. This pathway utilizes exocytosis and endocytosis of vesicles to deliver secretory and membrane proteins from the ER to the Glogi and on to secretory vesicles that are targeted to the cells exterior. After translation at the ER, proteins are transported to the Golgi apparatus. The Golgi apparatus is a major site of carbohydrate synthesis and is involved in sorting the proteins from the ER. It consists of an ordered series of compartments from the cis Golgi, the site of entry, through the medial Golgi to the trans Golgi, 
the exit site. The exact mechanism of transport between each Golgi compartment is unknown, but is thought to be either by vesicle transport or maturation of the compartments. What is known is that oligosaccharide processing is organized into separate compartments. Oligosaccharides that are linked to the hydroxyl group of serine, threonine or hydroxylysine are formed in the Golgi apparatus by unknown mechanisms. Another important process of the Golgi apparatus is to separate plasma membrane proteins from the membrane proteins with functions in the ER and Golgi apparatus. The lipid bilayer of the plasma membrane is thicker because it is rich in cholesterol. The rigid sterol ring of cholesterol forces neighboring hydrocarbon chains into more extended conformations, increasing membrane thickness (GarciaSaez et al., 2007). Membrane proteins destined for the plasma membrane have longer transmembrane segments (20-25aa) to span the thickness, while proteins with shorter transmembrane segments (15aa) are excluded. Once membrane proteins are packaged into vesicles they are transported to the membrane through constitutive or regulated mechanisms.

In the plasma membrane it is imperative that certain proteins be restricted to specific regions of the cell. In neurons that have polarized morphologies this is taken to an extreme. In order for electrical signals to be correctly processed by the neuron they must be received by its dendritic processes, relayed to the cell body where the output is then conveyed to the next cell via the axon terminal. Functional specializations require a high degree of molecular compartmentalization. To establish and maintain polarity, membrane proteins are sorted into distinct vesicles for proper transport. Most studies of vesicle packaging come from the apical and basolateral division of epithelial cells (Folsch, 2008). Proteins in polarized epithelial cells contain sorting signals in their cytoplasmic tails, which ensures they are packaged into vesicles destined for either the apical or basolateral plasma membrane (Ang et al., 2004; Bonifacino \& Traub, 2003; Luton et al., 2009). In neurons, a few channels, for example the shaker Kv1 voltage- 
gated potassium channel, have been shown to contain amino acid motifs that direct their axonal or dendritic targeting; however, the mechanism is unknown (Gu et al., 2003; Rivera et al., 2003; Kanaani et al., 2002). Though neuronal sorting of proteins remains enigmatic, the molecular trafficking of vesicles between axons and dendrites displays distinct mechanisms.

In axons and dendrites microtubules serve as scaffolds along which vesicles and macromolecular complexes can be transported. Microtubules are highly dynamic, polar cylinders made from a polymer of $\alpha$ - and $\beta$-tubulins. In axons and distal dendrites the positive end of the microtubule points away from the cell body, while proximal dendrites contain microtubules of mixed polarity (Burton \& Paige, 1981; Bass et al., 1988). Microtubule-associated proteins (MAPs) associate with microtubules to control their dynamics. Axons and dendrites express different MAPs, which determine the spacing between microtubules (Chen et al., 1982). Axonal microtubules express the MAP, tau, and are typically $20 \mathrm{~nm}$ apart, where as dendritic microtubules express MAP2 and are typically $65 \mathrm{~nm}$ apart (Chen et al., 1992; Kanai \& Hirokawa, 1995). Stable microtubules undergo detyrosination of the C-terminal tyrosine of $\alpha$-tubulin, which is removed through the action of tubulin carboxypeptidase (Ersfeld et al., 1993). Microtubules in the axon are highly stable and tend to be detyrosinated, while dendritic microtubules are more dynamic and remain tyrosinated (Khawaja et al., 1988; Witte et al., 2008; Konishi et al., 2009).

Vesicle trafficking is mediated by motor proteins, which distinguish different microtubules and leads to transport to either the axonal or dendritic compartment (Burack et al., 2000; Setou et al., 2000; Hirokawa \& Takemura, 2005). Certain kinesin motors (KIF17 and KIFC2) interact specifically with dendritic proteins and are confined to the dendrites (Setou et al., 2000; Saito et al., 1997). However, the motor domains of these kinesins do not appear to have a preference for dendritic versus axonal microtubules, suggesting that vesicular targeting 
may depend on additional cues (Nakata \& Hirokawa 2003). Recent evidence suggests that this cue is the cytoskeletal structure of the axon which acts as a molecular sieve. A network of polarized actin filaments in the initial segment of the axon prevents dendritic vesicles from passing into axonal areas (Song et al., 2009; Lewis et al., 2009). Dendritic vesicles associate with both Kinesin and Myosin Va motors. The Kinesin motors move vesicles along microtubules; however, when Myosin Va interacts with the actin filaments in the proximal axon the vesicle is retargeted back to the cell body for proper trafficking to the dendrite. Vesicles targeted to the axon do not contain Myosin Va. In addition, the ability of a vesicle to pass through the axon filter depends on which kinesin isoform is propelling it along with the identity of the protein (Song et al., 2009). This new hypothesis of an axonal filter has advanced our understanding of neuronal transport of vesicles; however, many questions regarding vesicle packaging remain. 


\section{CHAPTER II. DETERMINE THE EXPRESSION PROFILE OF TRPM1 IN THE RETINA}

Hypothesis The TRPM1 channel is expressed at the tips of the dendrites of DBCs and co-localizes with nyctalopin.

\section{Introduction}

Synaptic transmission from photoreceptors (PRs) to depolarizing bipolar cells (DBC) is mediated by the metabotropic glutamate receptor 6, Grm6 (Nakajima S., 1993; Nomura et al., 1994). Tonic activation of Grm6 in the dark results in closure of a non-specific cation channel, which keeps DBCs hyperpolarized (Shiells et al., 1981; Slaughter \& Miller, 1981). There is a sign reversal at the PR to DBC synapse, which inverts light-activated hyperpolarization of PRs into a depolarization in DBCs.

The Grm6 receptor is a member of the group III family of metabotropic glutamate receptors, which include: Grm4, Grm7 and Grm8 (Conn \& Pinn, 1997). In general these group III receptors are coupled to the inhibition of adenylyl cyclase and decrease in cAMP (Okamoto et al., 1994). The mechanism by which Grm6 modulates the non-selective cation channel in DBCs remains unknown (see review by Snellman et al., 2008).

Until recently, the molecular identity of the DBC cation channel was unknown. Two general candidates were postulated: a cyclic-nucleotide-gated (CNG) channel or a transient receptor potential (TRP) channel (Nawy \& Jahr, 1990; Snellman \& Nawy, 2004). A CNG channel was proposed because exogenous cGMP potentiated the Grm6 responses to glutamate and light 
(Nawy \& Jahr, 1990; Shiells \& Falk, 1990, 2002; de la Villa et al., 1995). This is a similar cascade to phototransduction, so the theory gained rapid acceptance in the literature. Though CNG channel mRNA was detected in goldfish DBCs by single cell RT-PCR and in situ hybridization, immunocytochemical identification in mammalian DBCs was negative (Wässle et al., 1992; Henry et al., 2003). Additionally, dialysis of DBCs with poorly hydrolyzed analogs of cGMP did not alter the glutamate response and addition of cGMP in the presence of glutamate had no effect on the cells holding current (Nawy, 1999; Snellman \& Nawy, 2004). These results indicate that cGMP alone cannot gate the channel. The lack of evidence for a CNG channel turned the focus to the TRP channels.

TRP channels are a large family of nonselective cation channels. TRP channels were proposed to be the transduction channel in DBCs because they are desensitized by calcium, can be modulated by G-protein signaling and the transduction currents had a similar pharmacological profile to several classes of TRP channels (Snellman et al., 2008). Initial candidates from the TRP channel super-family were TRPV1 and TRPM1. TRPV1 is expressed predominantly in the peripheral nervous system and mediates heat sensation. It was selected as a candidate because it had several properties in common with the DBC channel, including permeability to and desensitization by calcium (Liu \& Simon, 1996; Caterina et al., 1997; Koplas et al., 1997; Nawy, 2000; Shiells \& Falk, 1999; Berntson et al., 2004; Nawy, 2004). In addition, the DBC transduction channel could be activated by both capsaicin and anandamide, compounds known to activate TRPV1 (Shen et al., 2009; Caterina et al., 1997; Piper et al., 1999). Interest in TRPM1 as a candidate arose when TRPM1 mRNA was identified in a subset of DBCs (Kim et al., 2008). Additionally, decreased expression of TRPM1 mRNA was proposed as the cause of congenital stationary night blindness (CSNB) in Appaloosa horses (Bellone et al., 2008). 
TRPV1 and TRPM1 knockout mice were examined to determine if inactivation of these genes impacted DBC function (Shen et al., 2009). The DBC transduction channel is required for proper signal transmission and its loss would be expected to result in a no b-wave ERG phenotype. TRPV1 $\%$ mice had a normal ERG suggesting they retained the DBC transduction current (Shen et al., 2009). In contrast, TRPM1 ${ }^{-}$mice lacked an ERG b-wave indicating the TRPM1 channel is required for normal function of DBCs (Figure 6A and B, Shen et al., 2009). The ERG recordings suggested TRPM1, not TRPV1, is playing a critical role in DBC signaling.
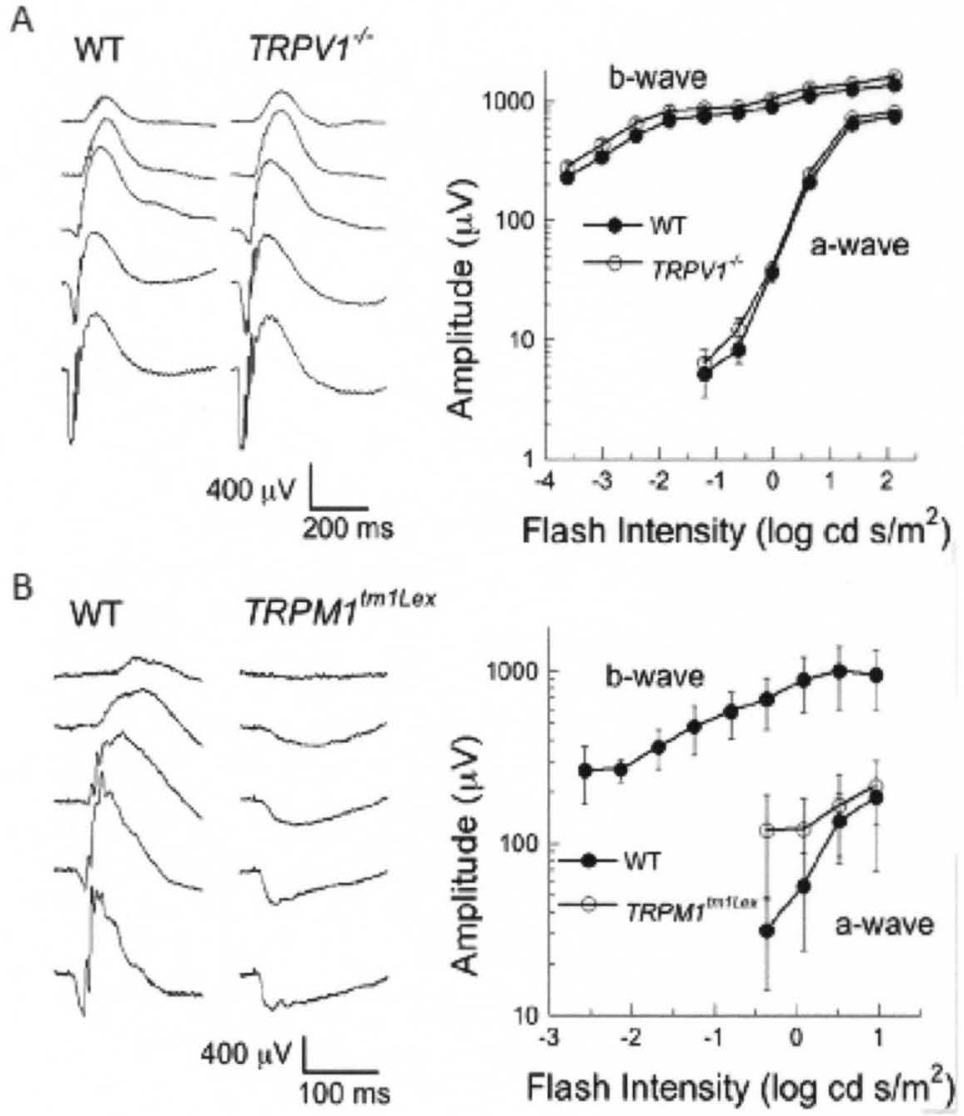

Figure 6: Analysis of TRPM1 and TRPV1 knockout mouse ERGs. (A) Left, dark-adapted ERGs from a wildtype (WT) and a TRPV1 $\%$ mouse. Flash intensities are -3.6, -2.4, -1.2, 0.0 and $1.4 \log \mathrm{cd} \mathrm{s} / \mathrm{m}^{2}$. Right, summary of the b-wave and a-wave amplitudes as a function of light intensity in WT $(n=8)$ and TRPV1 $1^{-}$ $(n=6)$ mice. (B) Left, dark-adapted ERGs from a WT and a TRPM1 $1^{--}$mouse. Flash intensities are -2.6, -1.7, $0.8,0.1$ and $1.0 \log \mathrm{cd} \mathrm{s} / \mathrm{m}^{2}$. Right, summary of the b-wave and a-wave amplitudes as a function of light intensity in WT $(n=3)$ and TRPM1 ${ }^{-/}(n=4)$ mice. For TRPM1 ${ }^{-}$, only the a-wave data are plotted, as there was no measurable positive-going response. Adapted from Shen et al., 2009. 
Individuals with CSNB1 have a defect in signaling from PR to DBCs, which is characterized by an absent b-wave and normal a-wave in the ERG. CSNB1 is caused by postsynaptic defects in DBC signaling. Mutations in NYX and GRMG have been shown to be causative of CSNB1 in many individuals (Bech-Hansen et al., 2000; Pusch et al., 2000; Jacobi et al., 2002; Zeitz et al., 2005a, 2005b; Dryja et al., 2005). However, several CSNB1 individuals did not have mutations in these genes. Three groups simultaneously investigated if mutations in TRPM1 might be responsible. The majority ( 50\%) of autosomal recessive CSNB1 (arCSNB1) probands contain mutations in TRPM1 (van Genderen et al., 2009; Ando et al., 2009; Li et al., 2009). Patch clamp recordings from $T R P M 1^{--}$mice also show the lack of a light-evoked response, confirming the defect is in DBC signaling (Koike et al., 2009). Additionally, immunohistochemistry localized TRPM1 to the DBC dendritic tips in human, mouse and monkey retina sections (van Genderen et al., 2009; Morgans et al., 2009; Koike et al., 2009). Collectively, these studies established that the TRPM1 channel is required for the transduction current in DBCs.

The current murine Ensembl database (www.ensembl.org) contains twenty splice variants of TRPM1, with thirteen predicted to be protein coding. The high number of TRPM1 splice variants raises questions about their expression profile and function. TRP channels are known to form hetero-tetramers, so alternative isoforms could come together to form novel channels. Additionally, studies in HEK293T cells have shown that a short TRPM1 splice variant, containing no transmembrane domains, suppresses function of the long channel forming variant (Xu et al., 2002). Whether a single or multiple TRPM1 splice variants are required for DBC signaling is currently unknown.

The idea that $D B C$ isoform specific gene expression occurs is expected. $G \alpha_{0}$ is part of the heterotrimeric G-protein complex that is activated by glutamate binding to the Grm6 receptor 
(Dhingra et al., 2000). The $G \alpha_{0}$ subunit has two splice variants in the retina, referred to as $G \alpha_{0} 1$ and $G \alpha_{0} 2$. Targeted deletion of $G \alpha_{0} 1$ causes a no b-wave ERG phenotype, while $G \alpha_{0} 2$ knockout mice have a normal ERG response. Therefore, only $\mathrm{G} \alpha_{0} 1$ is believed to be involved in signal transduction from Grm6 in the DBCs (Dhingra et al., 2002). One goal of my studies was to characterize the TRPM1 isoforms expressed in the retina.

Retinal genes have a well-defined temporal expression profile during development that correlates with cell differentiation and the formation of synapses (Cepko et al., 1996). In the vertebrate retina there are five major neuronal cell classes and one glial cell type that develop in an overlapping and well-defined temporal pattern (Cepko et al., 1996). In mice, ganglion cells, cone PRs, ACs, and HCs are specified embryonically, and BCs and Müller glia specified postnatally (Sidman 1961; Young 1985a, 1985b). Rod PRs, the most abundant cell type in the retina, are born both pre- and postnatally, with the peak coinciding with the day of birth (Cepko et al., 1996). Birthdating studies have given rise to the competence model of retinal development, in which the ability of a retinal progenitor cell to produce a particular cell type changes through out development (Cepko et al., 1996). Retinal progenitors have the potential to produce all the retinal cell types during retinal development. However, they only produce a single cell, or a subset of cells, at any one time. This has provided a means to correlate developmental gene expression with individual cell type expression.

When I joined the Gregg lab the identity of the DBC cation channel remained unknown. As my dissertation progressed TRPM1 was identified as the non-selective cation channel. This chapter describes my work to characterize TRPM1 expression in the retina. 


\section{Results}

\section{Molecular Cloning of TRPM1 from Retina}

To determine if TRPM1 and/or TRPV1 was expressed in the retina I used reverse transcriptase PCR (RT-PCR) to amplify cDNAs from C57BL/6J retina RNA. The Ensembl database indicated that full-length mouse TRPM1 has a 4,869 base pair (bp) open reading frame (ORF) and a 142bp 5' untranslated region (UTR) (TRPM1; ENSMUST00000085222). The TRPV1 cDNA has 3,252bp ORF with a 109bp 5'UTR (TRPV1; ENSMUST00000102526). Primers were designed that would amplify the full length cDNA representing each gene's ORF (Table 1). RT-PCR for fulllength TRPM1 from retinal cDNA yielded fragments of approximately 4,800bp (Figure 7A). Multiple primer sets were tested for TRPV1 (Table 1), but I was unable to obtain any evidence it was expressed in murine retina. Molecular cloning of TRPM1 from retinal CDNA identified several splice variants. I characterized the mRNA expression profile of these TRPM1 splice variants in the retina to determine which variant(s) would likely be essential to DBC function.

Table 1. PCR primer sets for TRPM1 and TRPV1 in murine retina

\begin{tabular}{lll}
\hline PCR Name & Forward Primer* & Reverse Primer* \\
\hline TRPM1 & CCCATGGGGTCCATGAGGAA & TCAGCACTCAGTTTCCGCGC \\
TRPM1-005 & GAGGAGGCTGAAGGAGGCTA & GCGCGGAAACTGAGTGCTGA \\
TRPM1-007 & GAGGAGGCTGAAGGAGGCTA & TCAGGGCAGAGGTGGGGTGA \\
TRPM1-008 & GAGGAGGCTGAAGGAGGCTA & GTGTGTTCACTTCAATGTAG \\
FL-TRPV1 & CCCATGGAGAAATGGGCTAGCTTAG & TCATTCTCCCCTGGGGCCATGGAAT \\
5'-TRPV1 & CCCATGGAGAAATGGGCTAGCTTAG & GTGTCACTACGGCTGTGGAA \\
3'-TRPV1 $^{\prime}$ TGTACTTCAGCCATCGCAAG & TCATTCTCCCCTGGGGCCATGGAAT \\
\hline
\end{tabular}

'All primers are listed from 5' to 3'. 
PCR products from two RT-PCR reactions were cloned into the PCR-Blunt II-TOPO plasmid. 9 and 13 clones from the two cloning reactions, respectively, were analyzed further. Sequence analysis of the 22 clones identified four splice variants: TRPM1, TRPM1-LF, TRPM1-N and TRPM1-SV, shown schematically in Figure 7B. Table 2 summarizes the retinal variants and describes their insertions or deletions compared to the full length TRPM1 (Accession, NM_018752). TRPM1-SS and TRPM1-LF matched variants reported in the mouse Ensembl database. However, TRPM1-SV and TRPM1-N are novel variants.

A full length TRPM1 variant, TRPM1-N, was cloned from a $\mathrm{CH} 3 / \mathrm{HeJ}$ strain of mouse. This variant was identified during a screen to determine the gene responsible for a spontaneous mutant $\mathrm{CH} 3 / \mathrm{HeJ}$ mouse with a no b-wave ERG phenotype, now called nob5. Whether the TRPM1-N variant is expressed in $C 57 B L / 6 J$ retina remains to be determined.

In addition to full length TRPM1 isoforms, short forms lacking the transmembrane domains have been reported and are represented in the mouse Ensembl database. Most of the short variants of TRPM1 contain a different 5' untranslated region (UTR) and alternative downstream start sites. Primers also were generated to amplify these variants. Only one short isoform, TRPM1-007, was amplified from the retinal cDNA (Figure 7A, ENSMUST00000107516, Table 2).

Table 2: TRPM1 retinal splice variants.

\begin{tabular}{llcccl}
\hline Name & Ensembl & BP & ORF & AA & Insertions/Deletions \\
\hline TRPM1-SS & TRPM1-003 & 5013 & 4869 & 1622 & None \\
TRPM1-LF & TRPM1-001 & 5076 & 4887 & 1628 & RKTRIH inserted at 1065aa between Exon 23 \& 24 \\
TRPM1-SV & NONE & 4481 & 4737 & 1578 & Deleted Exon 20 \\
TRPM1-N & NONE & 5040 & 4896 & 1631 & VSIPGGRVE inserted at 549aa between Ex on 13 \& 14 \\
TRPM1-007 & TRPM1-007 & 2765 & 1629 & 547 & $\begin{array}{l}\text { 5'UTR missing Exon 1, but contains Exon 2. ATG start site in Exon } 4 \\
\text { VELSRHTVSCASOSNMWFLDVLOKPTCAECNSSPHLSOTDITPPLP. } \\
\end{array}$ \\
& & & & inserted at 611aa between Exon 15 \& 16
\end{tabular}




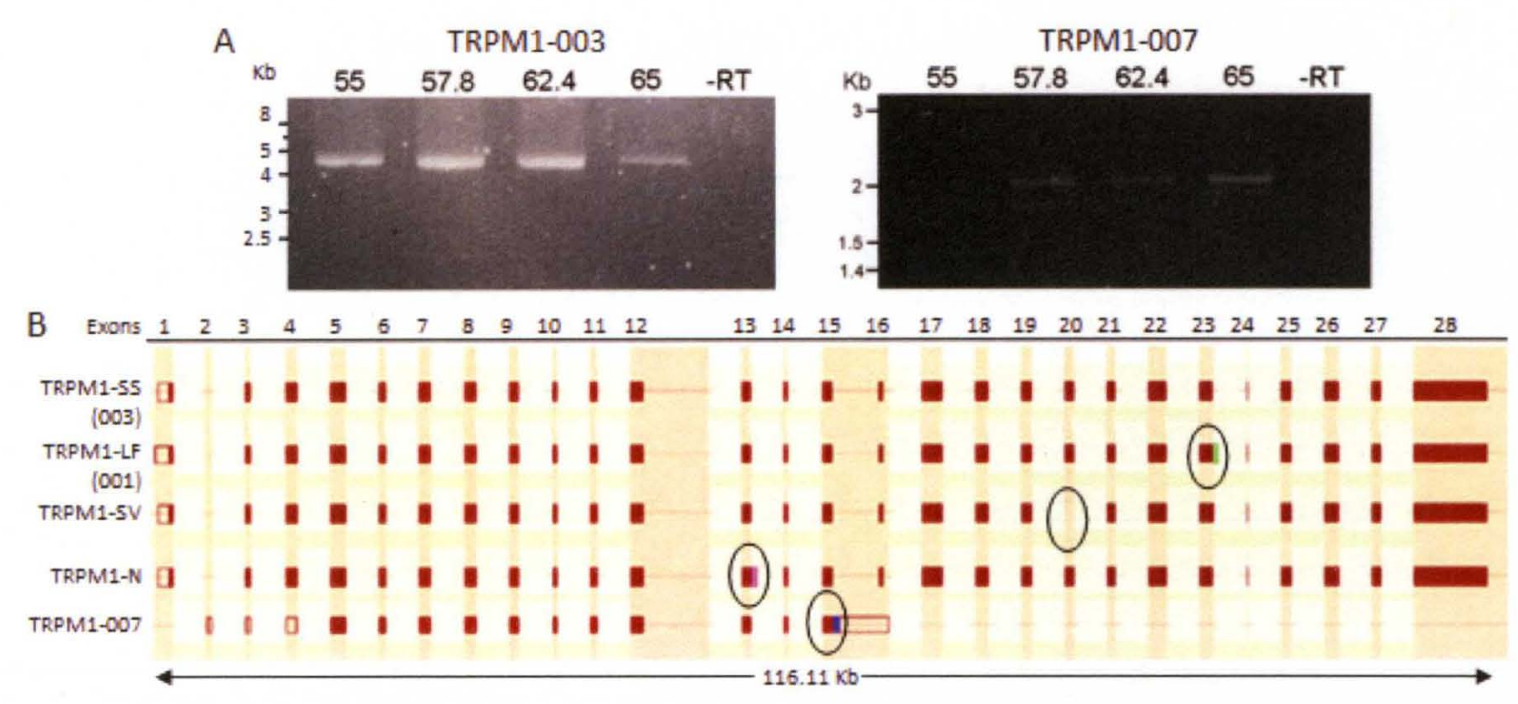

Figure 7: Analysis of TRPM1 in retinal cDNA. (A) Cloning for full length TRPM1-003 and TRPM1-007 from retinal cDNA library. (B) Schematic of the exons, depicted with red boxes, used for each retinal TRPM1 splice variant. Intronic additions are shown for TRPM1-LF in green, TRPM1-N in pink and TRPM1-007 in purple.

\section{Multiple TRPM1 Splice Variants Expressed in Murine Retina}

I identified five retinal TRPM1 splice variants that all could produce a functional TRPM1 channel. To determine which isoform(s) are likely to form a functional channel in the DBC dendrites I examined the expression profile of each during development. Ideally this would also be corroborated using isoform specific antibodies to show protein distribution; however, the only available antibodies recognize all full length variants (Morgans et al., 2009; Koike et al., 2009).

Many genes expressed in BCs have been shown to exhibit a specific expression profile during development (Kim et al., 2008). This BC profile shows low expression levels at P0 with expression gradually increasing and peaking at P13-21, after which expression declines (Kim et al., 2008). This developmental expression pattern correlates with the terminal differentiation of $\mathrm{BC}$ s and synaptogenesis between PR axons and $\mathrm{BC}$ dendrites in the $\mathrm{OPL}$. The $\mathrm{BC}$ specific genes used to generate the expression profile were from different $B C$ cell subtypes, so specific $B C$ types (even differences between DBCs and HBCs) cannot be identified. However, determining 
gene expression can be used to differentiate BC-enriched genes from genes enriched in the PRs, ACs or GCs.

The temporal expression pattern of TRPM1 was first described after microarray analyses of retina cDNA (Kim et al., 2008). Its expression follows a BC developmental pattern and in situ hybridization confirmed its expression was restricted to BCs (Kim et al., 2008). The isoform analyzed in these studies was not identified. To determine the temporal expression pattern of TRPM1 isoforms during development I used isoform specific quantitative real-time PCR (qRTPCR).

Specific Taqman assays were designed to distinguish the different TRPM1 splice variants that are predicted to contain transmembrane domains, TRPM1-SS, TRPM1-LF, TRPM1-N and TRPM1-SV. To quantify total TRPM1 expression I designed a Taqman assay, TRPM1-T, targeting the exon 3 and 4 junction, which is present in all isoforms. Taqman primers to discriminate TRPM1-LF and TRPM1-N could not be designed within the small intronic insertions. I designed Taqman assays specific to TRPM1-SV and the TRPM1-SS, TRPM1-LF and TRPM1-N variants (TRPM1-LF). The TRPM1-SV assay flanks the exon 19-21 junction that is present only in TRPM1-SV. The TRPM1-LF Taqman assay was designed to flank the exon 20-21 junction, which is absent in TRPM1-SV and present in TRPM1-SS, TRPM1-SV and TRPM1-N. The location of these Taqman assays is shown schematically on the TRPM1 transcript in Figure 8A.

Initial experiments were performed to check the specificity of the Taqman assays for their respective TRPM1 transcripts. To quantify the absolute amount of each transcript I generated a standard curve using known concentrations of plasmid DNA representing each isoform. Plasmids containing cDNA clones of TRPM1-LF or TRPM1-SV were serially diluted over a 9-log range. To increase the complexity of the cDNA pool I added 50ng of cDNA synthesized from HEK293T cells, which do not express TRPM1. Figure 8B shows the standard curves for each 
Taqman assay, plotted as $\log$ of the concentration of each isoform $(\log [\mathrm{pg}])$ verse cycle number (Ct). TRPM1-T assay identified both the TOPO-TRPM1-LF (red, Figure 8A) and TOPO-TRPM1-SV (blue, Figure 8A) plasmids with similar efficiency, as indicated by the overlap between the templates at each concentration. The TRPM1-LF assay detected TOPO-TRPM1-SV plasmid, but 64 fold less efficiently. The TRPM1-SV assay amplified products from TOPO-TRPM1-LF plasmid, but only at concentrations close to $1 \mathrm{ng}$, which is not biologically relevant (Figure $8 \mathrm{~B}$ ). These data show that the TRPM1 Taqman assays can be used to distinguish TRPM1 variants; TRPM-SS, TRPM1-LF and TRPM1-N from TRPM1-SV. Once I established the utility of these assays I used them to determine the developmental profile of the TRPM1 splice variants in the retina. In addition, the total amount of TRPM1 CDNA and the amount of each variant in the retina was quantified using calculated standard curves. 


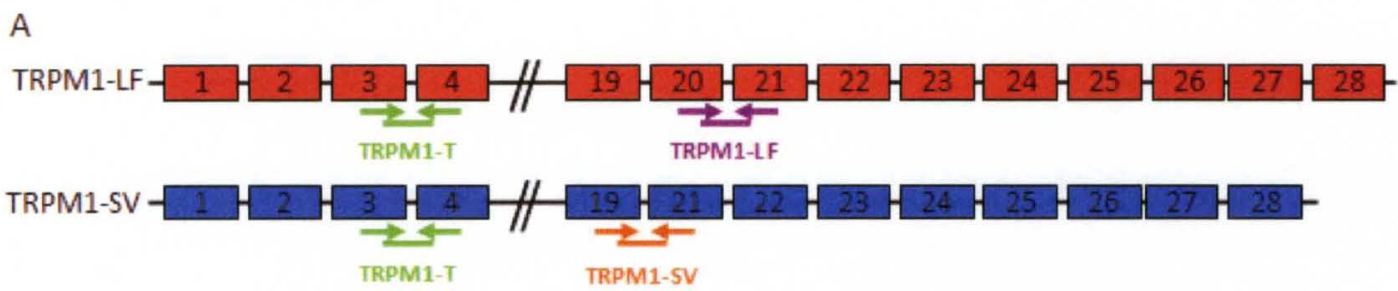

B

TOPO-TRPM1-LF

TOPO-TRPM1-SV

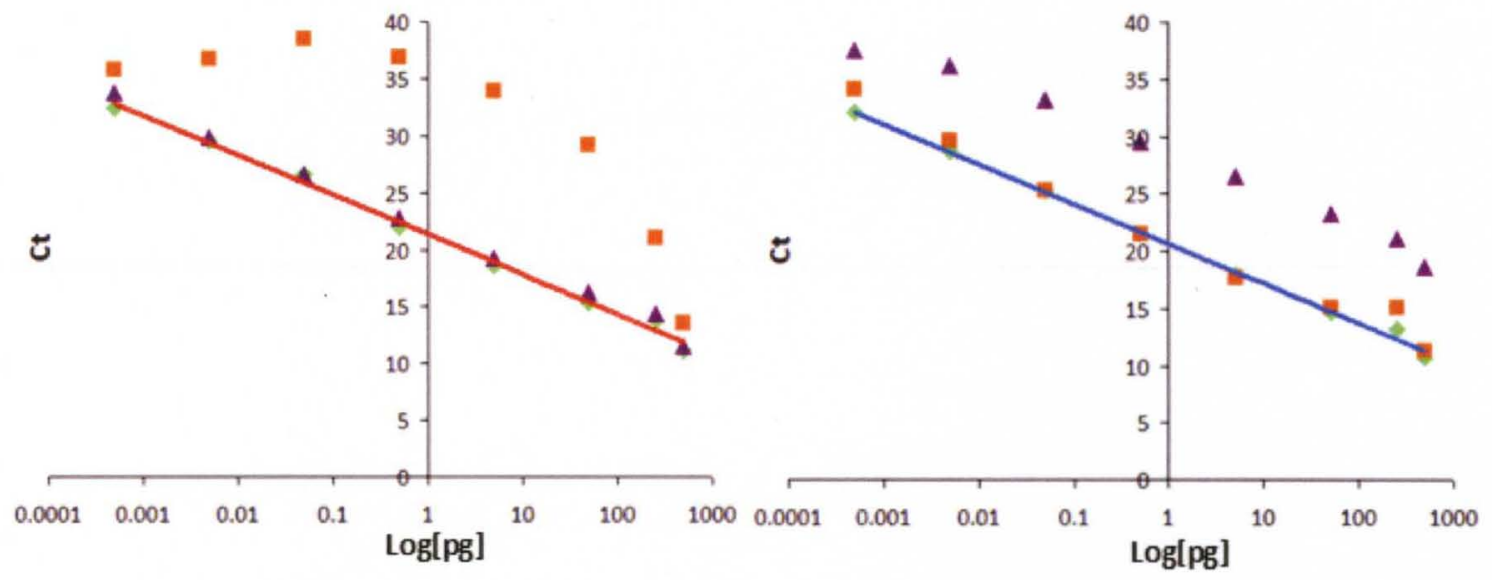

Figure 8: Analysis of TRPM1 Taqman assays. (A) Schematic showing the location of TRPM1 Taqman assays on TRPM1-LF and TRPM1-SV templates. Total TRPM1 (green, TRPM1-T), long forms of TRPM1 (purple, TRPM1-LF) and the splice variant of TRPM1 (orange, TRPM1-SV). (B) Standard curves generated for each the Taqman assays over a 9-log dilution series of a TOPO plasmid containing either TRPM1-LF (red) or TRPM1-SV (blue). The TRPM1-T assay was able to detect both plasmids with similar accuracy. TRPM1-SV assay was able to reliably detect TOPO-TRPM1-SV at low concentrations, but not TOPO-TRPM1-LF. TRPM1-LF assay was only able to detect TOPO-TRPM1-LF in the normal range. 
The expression level of the TRPM1 variants was quantified at seven developmental time points (P1, P7, P13, P21, P26 and 3 Months). To correct for variation in mRNA isolation and cDNA synthesis all samples were normalized to $18 \mathrm{~S}$ and expressed as $\mathrm{pg} /$ input RNA.

The results show that TRPM1-LF (red) has a similar developmental expression profile as TRPM1-T (green). Both have low expression between P1 and P7 then peak during synaptogenesis between P13-21 before declining in adults, 3 months (Figure 9). This temporal expression profile is distinctive to BC genes (Kim et al., 2008). The TRPM1-SV expression was lower than TRPM1-T or TRPM1-LF had and reached a plateau by P21. This temporal profile is similar to that seen for PR specific genes (Rho, Gnat1, and Guca1a; Kim et al., 2008). The temporal profile of the long forms of TRPM1 (TRPM1-SS, TRPM1-LF, and TRPM1-N) mRNA expression is consistent with the expression established for BC specific genes.



Figure 9: TRPM1 splice variant mRNA expression profile in the developing $C 57 B L / 6 J$ retina. Total TRPM1 (green, TRPM1-T) has a BC expression profile with peak expression between P13 and P26. Long form TRPM1 variants, which contain all six transmembrane domains, also follow a BC profile (red, TRPM1-LF). TRPM1 variants lacking exon 20 have a late expressing PR profile (blue. TRPM1-SV). 
TRPM1 Expression Profile in Wildtype vs. Nyx ${ }^{\text {nob }}$ Retina

In $\mathrm{Nyx}^{\text {nob }}$ mice, electrophysiological recordings from DBCs are consistent with the absence the TRPM1 channel or its presence in a constitutively closed state (Gregg et al. 2007). To determine the consequences of the absence of nyctalopin on TRPM1 transcription I compared the developmental mRNA expression pattern of TRPM1 in C57BL/6J and $N y x^{n o b}$ retina.

I used the TRPM1-T Taqman assay to quantify total transcript levels at six time points (P1, P7, P13, P21, P26 and 3 months). Expression of TRPM1 in the C57BI/6J wildtype mice follows a BC developmental pattern that peaks at P13-21 (grey, Figure 10). In contrast the TRPM1 transcript levels are reduced in the $N y x^{\text {nob }}$ mutant at late stages of development as well as in adult retinas (black, Figure 9). TRPM1-T qRT-PCR results between $\mathrm{C56BI/6J}$ and $\mathrm{Nyx}{ }^{\text {nob }}$ were analyzed by a Student t Test and showed no significant differences between any developmental time point (Table 3).

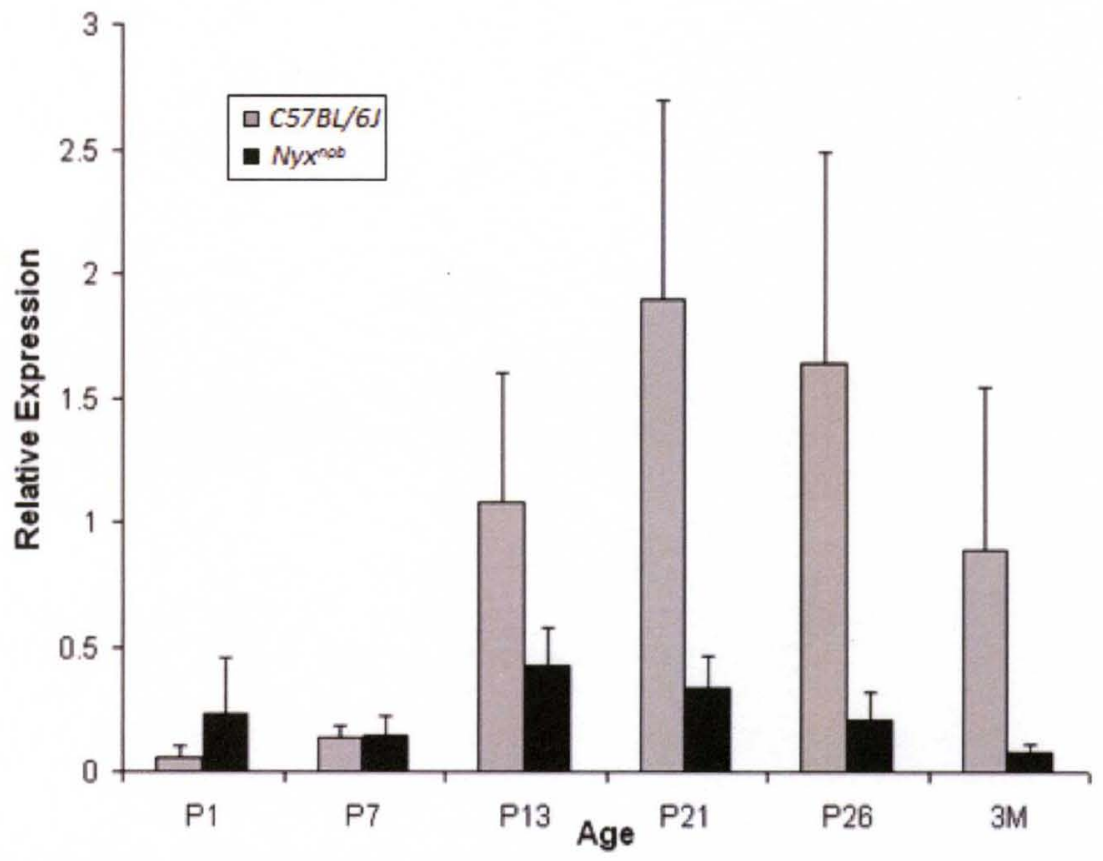

Figure 10: Total TRPM1 mRNA expression levels in C57BI/6J versus Nyxnob mice. In C57BI/6J mice TRPM1-T expression follows a BC expression profile, with peak expression at P21 (grey). In Nyx nob mice, the TRPM1-T mRNA levels are significantly reduced (black). Scale bars represent standard errors from four biological replicates per developmental time point. 
Table 5: $C 57 B 1 / 6 J$ verse $N y x^{\text {nob }}$ TRPM1-T qRT-PCR Statistics

\begin{tabular}{ccc}
\hline Age & T Value & T Probabiity \\
\hline P1 & -1.013 & 0.350 \\
P7 & -1.640 & 0.152 \\
P13 & -1.421 & 0.204 \\
P21 & -0.784 & 0.462 \\
P26 & 0.844 & 0.430 \\
3M & 0.612 & 0.562 \\
\hline
\end{tabular}

\section{Screening TRPM1 Antibodies}

To localize expression of the TRPM1 channel in the retina I tested six commercial TRPM1 antibodies (Table 4). These antibodies were made against human or mouse peptides. Each antibody was screened at three antibody dilutions (1:300, 1:1000 and 1:3000) on normal human and $C 57 B L / 6 J$ or $T R P M 1^{-}$mouse transverse retinal sections.

Table 4: Commercial TRPM1 antibodies screened for expression in the human and murine retina

\begin{tabular}{clcl}
\hline Lab \# & Company & Species & Antigen \\
\hline 84 & Santa Cruz (SC-16999) & Goat & Synthetic peptide from N-Terminus of human TRPM1 \\
85 & Novus (NB110-75005) & Sheep & Synthetic peptide from rat, mouse, dog and monkey TRPM1 (1066-1071aa) \\
86 & Novus (NB110-74963) & Rabbit & Synthetic peptide from mouse TRPM1(189-1078aa) \\
88 & Novus (NB110-74925) & Rabbit & Synthetic peptide from rat, mouse, dog and monkey TRPM1 (1066-1071aa) \\
89 & Novus (NB110-74972) & Sheep & Synthetic peptide from mouse TRPM1 (189-1078aa) \\
92 & Sigma (HPA014785) & Rabbit & Synthetic peptide from C-terminal of human TRPM1 \\
\hline
\end{tabular}

Along with TRPM1, retinal sections were stained with the OPL marker peanut agglutinin (PNA). PNA is a lectin with high affinity for galactose-galactosamine disaccharide residues. In the vertebrate retina, PNA labels cone PRs, specifically labeling their inner and outer segments and synaptic pedicles (Blanks \& Johnson, 1983, 1984). Cone synaptic pedicles make synapses with several hundred postsynaptic dendrites from BCs and HCs, so staining of the cone pedicle with PNA provides a marker for these synapses (Missotten, 1965; Chun et al., 1996; Haverkamp et al., 2000, Figure 11). Postsynaptic DBC proteins, such as Grm6 and nyctalopin, co-localize with PNA in the OPL at the light microscope level. Therefore, I expected TRPM1 to be expressed in DBCs and be restricted to the DBC dendritic tips and co-localize with the PNA marker. 

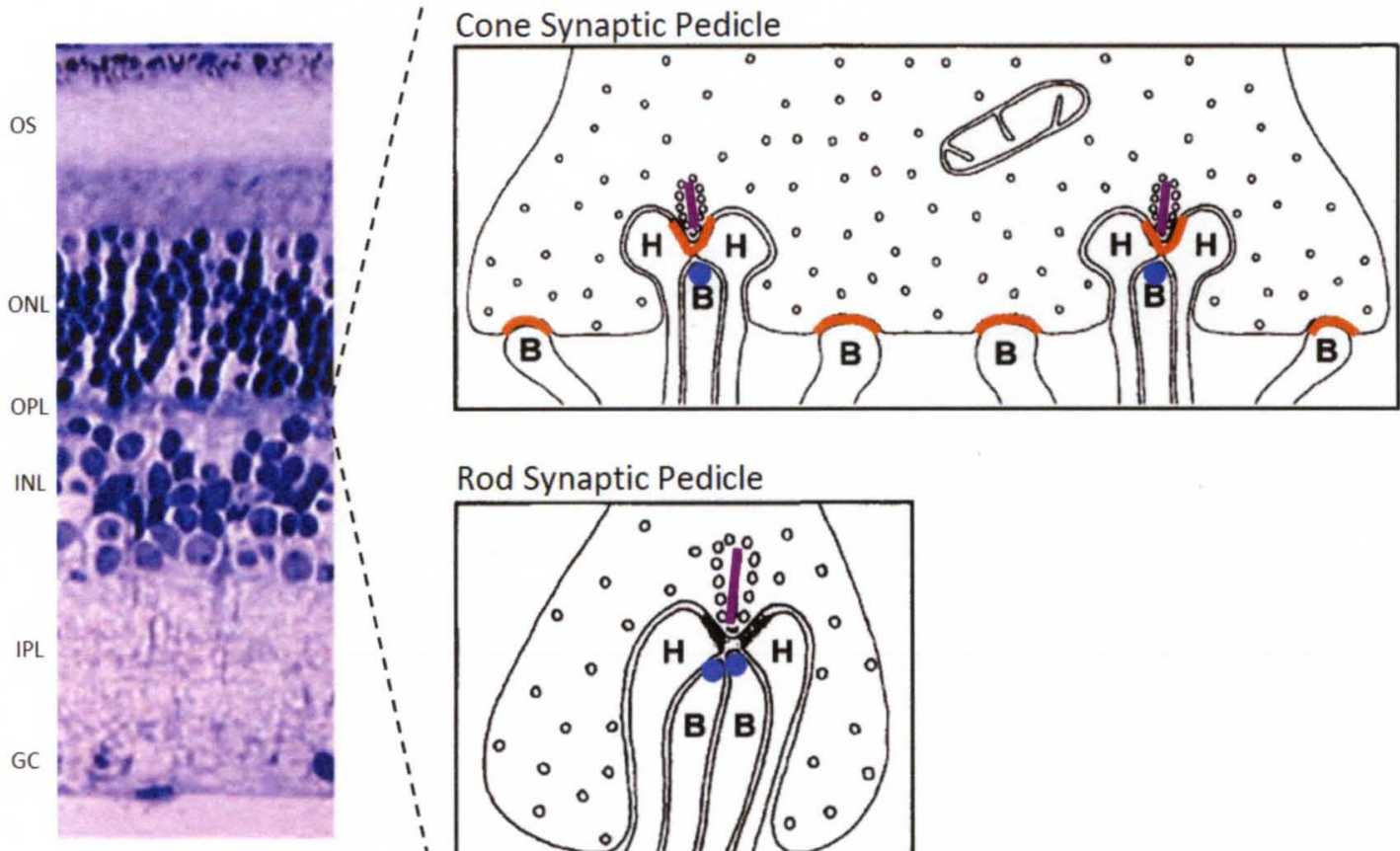

Rod Synaptic Pedicle

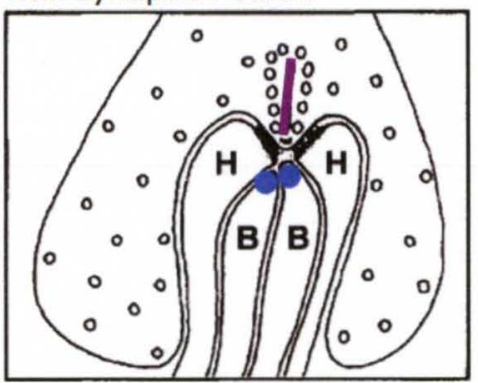

Figure 11: Immunohistochemical markers used to identify outer plexiform layer (OPL) of the retina. Vertical section of a creysl violet stained retina shows lamination of nuclear and plexiform layers. Enlarged cartoon image of the OPL where cone and rod PRs contact Horizontal (H) and Bipolar (B) cell dendrites. The PNA lectin, orange, labels the synaptic cleft in cone synaptic pedicles. RIBEYE antibodies, purple, label the ribbon synapse found in all PR axon terminals. Grm6 antibodies, blue, label the dendritic terminals of DBCs.

A representative image of each TRPM1 antibody tested in a C57BL/6J mouse retina at a 1:1000 dilution is shown in Figure 12A. Only the 92-TRPM1 antibody co-localized with PNA in the human and C57BL/6J mouse retinal sections. In addition, staining was absent in the TRPM1 ${ }^{-1}$ retina suggesting it is specific for TRPM1 (Figure 12B). The 92-TRPM1 antibody is made to a peptide from the C-terminus of the human TRPM1. Alignment of the human and murine amino acid sequences from this region shows the human peptide has $57.5 \%$ homology $(69 / 120$ conserved residues) with the mouse TRPM1 (conserved residues shown in red, Figure 12C). Though the antibody labeled TRPM1 in mouse retina I also evaluated whether this antibody could be used on western blots. Extracts from C57BL/6J and TRPM1\% mouse retina were isolated and analyzed. I was unable detect a specific band at the predicted size (180Kd). This antibody will be used for further immunohistochemical analysis of TRPM1 in the retina. 
A
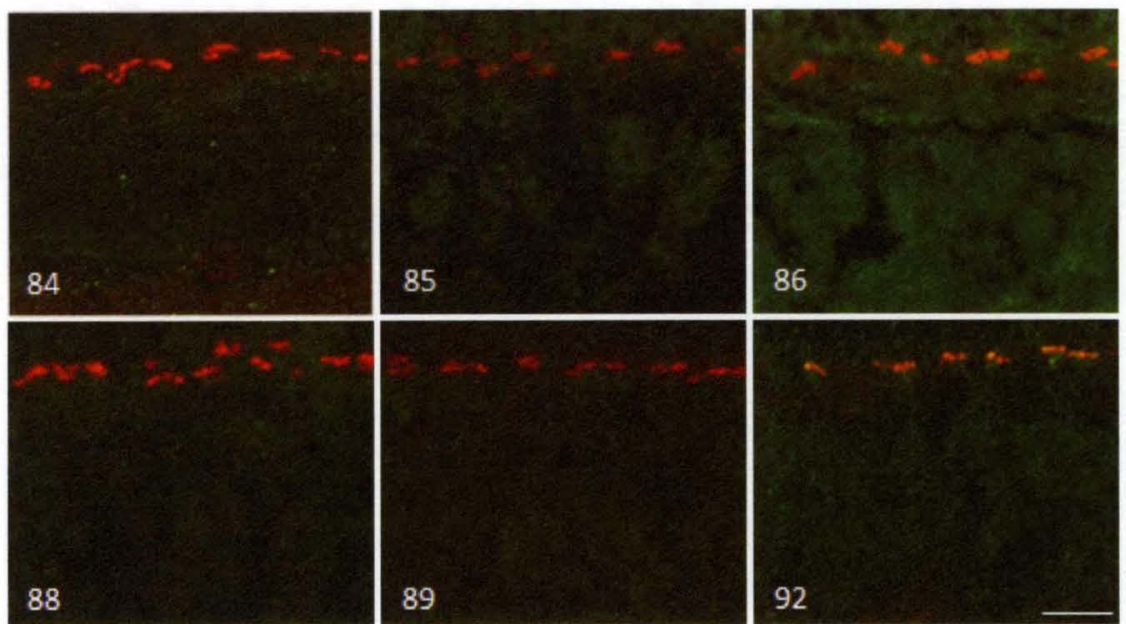

B $\quad 57 B \mid / 6 J$
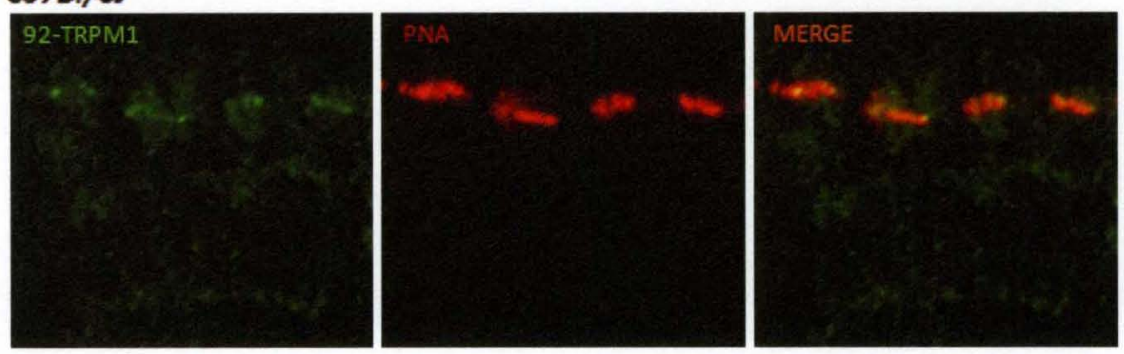

TRPM1\%
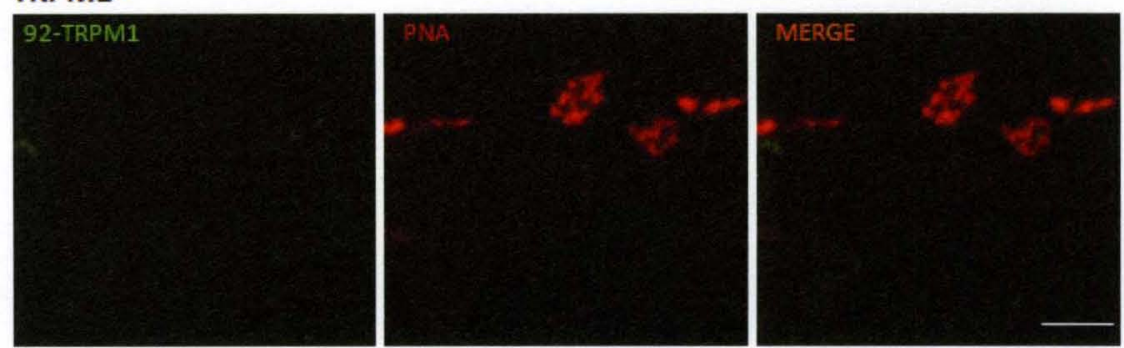

C

\begin{tabular}{l|l|l}
\hline & \\
\hline hTrpM1_pr_Q724N2 & VGGVN-QDVEYSSITDQQL-TTEWQCQVQKITRSHSTD IPYIVSEAAVQAE \\
mTrpm1_pr_Q2 TV84 & VRGLSNUSAEYSS IMDQA UNATEWRCQVQRITRSRSTD IPYIVSEAASQDE
\end{tabular}

HKEQF AD MQDE HHV AE A IPR I PRLSL TI TD RNGMENL LSVKP DQTL GF PSLRSKSL HGHP RNVKS IQGKL -- ---LEDEHRGSLLDPQISRS AL TVSDRPEKENLLSVKPHQTLGF PCLRSRSLHGRPRSAEP APSKL

Figure 12: Immunohistochemical analysis of TRPM1 antibodies in C57BI/6J retina. (A) Representative images for each TRPM1 antibody (84-86, 88, 89, 92, green) performed on C57BI/6J transverse retinal sections. The cone terminals in the OPL were localized using PNA (red). Antibodies were used at 1:1000 dilution of. Each image is a maximum projection of a $9 \mu \mathrm{m}$ z-stack. Scale bar is $10 \mu \mathrm{m}$. (B) Immunohistochemistry on $\mathrm{C} 57 \mathrm{BI} / 6 \mathrm{~J}$ and $T R P M 1^{-\%}$ retinal sections. 92-TRPM1 shows staining in the OPL of sections from $\mathrm{C} 57 \mathrm{BI} / 6 \mathrm{~J}$. There is no staining in sections from $\mathrm{TRPM}^{-1}$ mice. Scale bar is $5 \mu \mathrm{m}$. (C) Alignment of the human and mouse TRPM1 protein sequences in the region used to make the 92-TRPM1 antibody. Red are conserved sequences. 


\section{TRPM1 Expression in Human Retina}

Individuals with mutations in TRPM1 are night blind and have a CSNB1 ERG no b-wave phenotype, indicating disrupted PR to DBC signal transmission (van Genderen et al., 2009; Aldo et al., 2009; Li et al., 2009). In the previous section, I identified a TRPM1 specific antibody that identified expression of TRPM1 in the BC somas and the OPL. To further refine the expression pattern of TRPM1 I reacted transverse sections of normal human retina with antibodies to TRPM1 and either PNA or RIBEYE (Figure 13).

Peanut Agglutinin (PNA) stains the cone pedicles in the OPL and is known to co-localize with dendritic DBC proteins, such as Grm6 and nyctalopin (Nomura et al., 1994; Vardi et al., 2000; Gregg et al., 2007). Immunohistochemistry with TRPM1 antibodies and PNA show dense TRPM1 puncta in the OPL of normal human retina (Figure 13A). Large puncta co-localize with PNA and represent staining of the cone pedicles. To assess the co-localization quantitatively fluorescence intensity of green and red pixels was determined across a $2 \mu \mathrm{m}$ line placed at PNApositive puncta. These data show complete co-localization of TRPM1 and PNA (Figure 13C).

DBC dendrites form invaginating synapses with PR axon terminals. RIBEYE is a major component of retina synaptic ribbons found in the PRs axon terminals adjacent to the OPL synapse (Schmitz et al., 2000). Synaptic ribbons are important for the physiological properties of these tonically active synapses and it is thought that RIBEYE acts as a scaffold protein to build the entire ribbon (Magupalli et al., 2008). Staining for TRPM1 and RIBEYE show they are closely associated, but not overlapping, in both cone and rod terminals (Figure 13B). The cone terminals contain 30-40 DBC dendritic tips making them large puncta, while a single DBC dendrite contact one rod terminal so are smaller puncta. The RIBEYE staining surrounds the large and small TRPM1 puncta in the OPL (Figure 13B). The pixel fluorescence intensity for TRPM1 was determined across a $2.5 \mathrm{um}$ line placed at RIBEYE-positive puncta. The distribution of the pixel 
intensities across that line show that TRPM1 fluorescence does not co-localize with RIBEYE fluorescence, but is restricted to the DBC dendrites (Figure 13C).

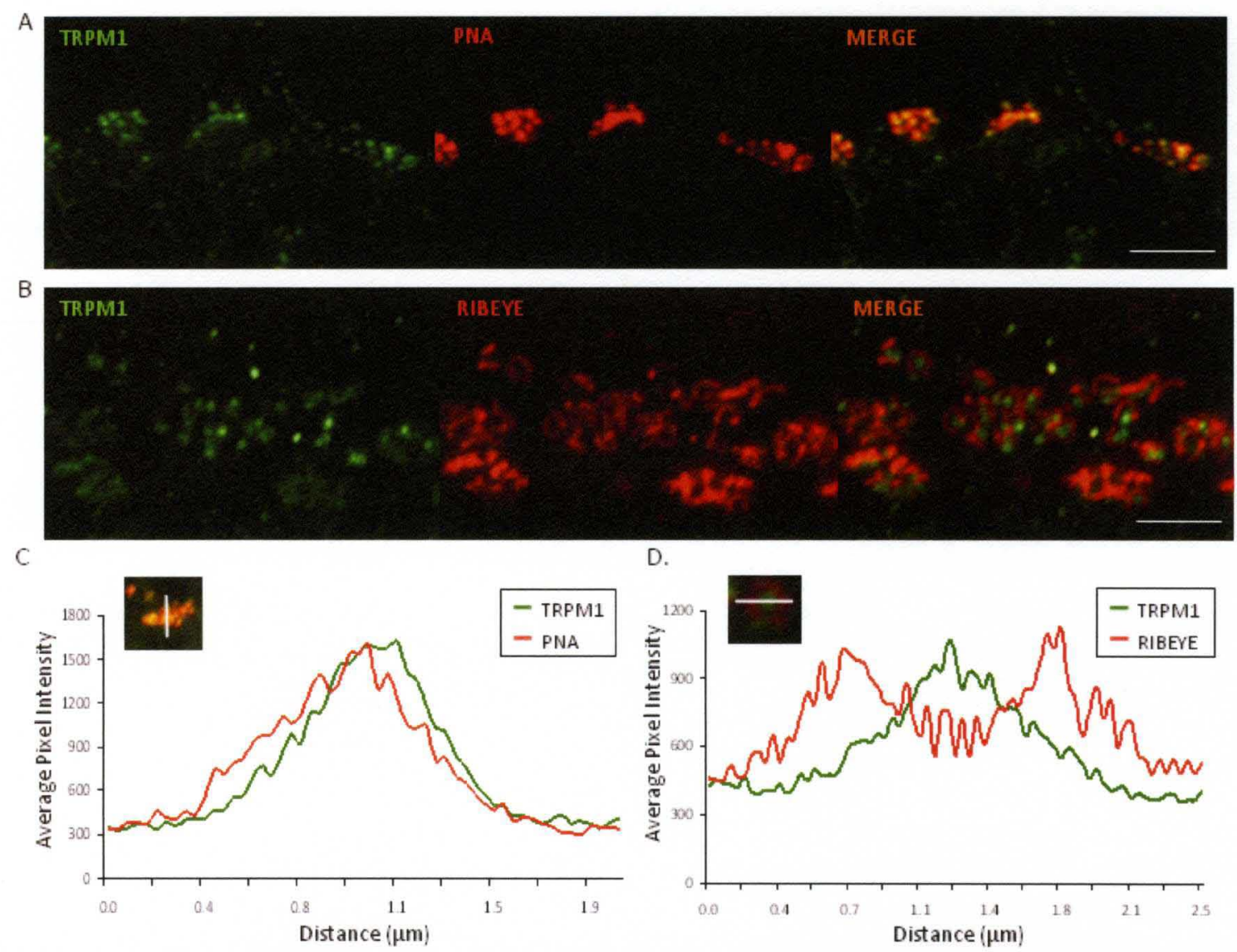

Figure 13: TRPM1 expression in human retina. (A) Immunohistochemical staining of human retinal sections for TRPM1 (green) and PNA (red). The merged images show that TRPM1 is co-localized with PNA. Scale bar is $5 \mu \mathrm{m}$. (B) TRPM1 staining is closely aligned with RIBEYE (red). Scale bar is $5 \mu \mathrm{m}$. (C) Quantitative analysis of pixel intensity for TRPM1 (green) and PNA (red). A line of $2 \mu \mathrm{m}$ (white bar) was drawn through the center of the distinct PNA-positive synapses. The distribution of the fluorescence intensity along this line was determined to generate fluorescent profiles corresponding to PNA (red) fluorescence and TRPM1 (green). (D) For quantification with RIBEYE a $2.5 \mu \mathrm{m}$ line was drawn through the center of the distinct RIBEYEpositive synapses. The distribution of the fluorescence intensity along this line was scanned to generate intensity profiles for TRPM1 (green) and RIBEYE (red). Shown traces are the average of 10 intensity measurements from different puncta within a single image. 


\section{TRPM1 Expression in Murine Retina}

To evaluate whether TRPM1 was similarly expressed in DBC dendrites at PR invaginating synapse, I reacted retinal sections with antibodies to both TRPM1 and PNA or RIBEYE. Figure 14A, shows representative expression patterns of TRPM1 and PNA in transverse retinal sections from $C 57 B L / 6 J$ mice. In the confocal merged image, co-localization of TRPM1 and PNA is evident and the pattern is consistent with alignment of the cone terminal with invaginating $\mathrm{BC}$ dendrites within the OPL. This co-localization as shown for human retinal sections also was quantified and I found that TRPM1 fluorescence overlaps with PNA fluorescence in the murine retina (Figure 14B). TRPM1 expression in the OPL was also examined in a whole mount retinal preparation (Figure $14 \mathrm{C}$ ). For these experiments $4 \mu \mathrm{m}$ confocal stacks were taken of the OPL. PNA positive cone pedicles co-localize with TRPM1 staining. I also examined transverse retinal sections for coexpression of TRPM1 and RIBEYE (Figure 14D). The merged images show that RIBEYE and TRPM1 do not co-localize, rather they are closely associated as in the human synapse. Quantification of the co-localization confirmed that TRPM1 does not co-localize with the RIBEYE-positive ribbon synapse (Figure 14E). TRPM1 expression in murine retina is consistent with human retina at the immunohistochemical level. 
A

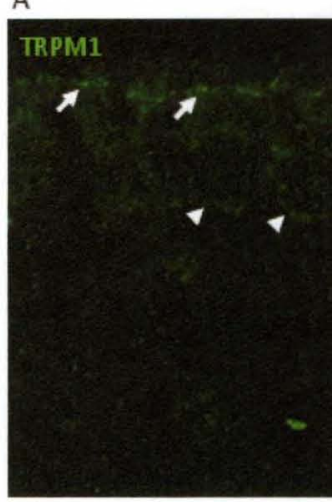

C

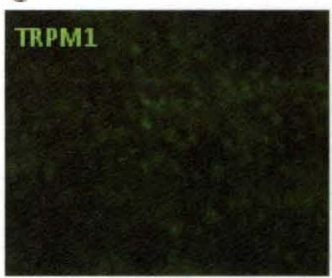

D

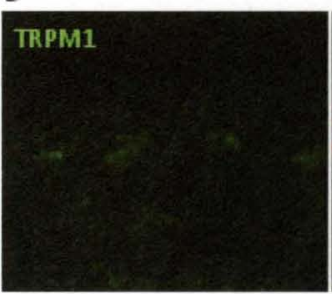

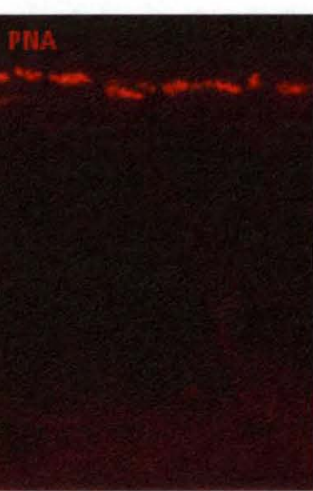
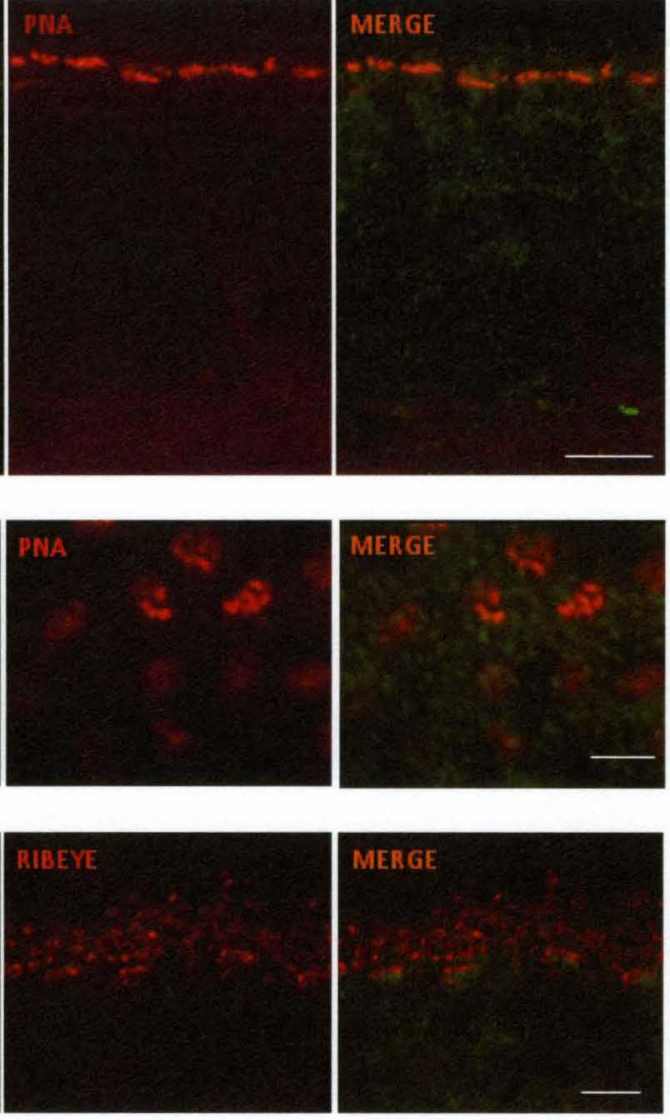

B
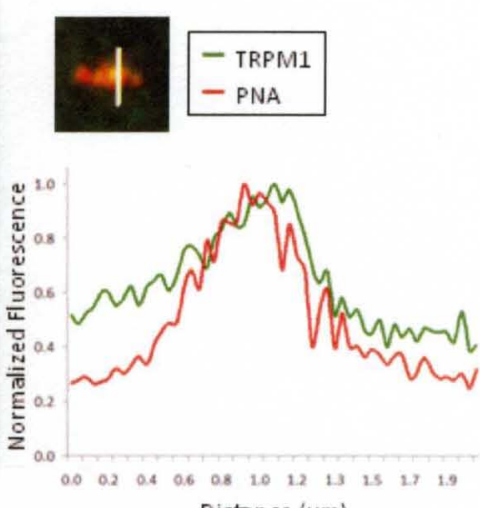

Distance $(\mu \mathrm{m})$
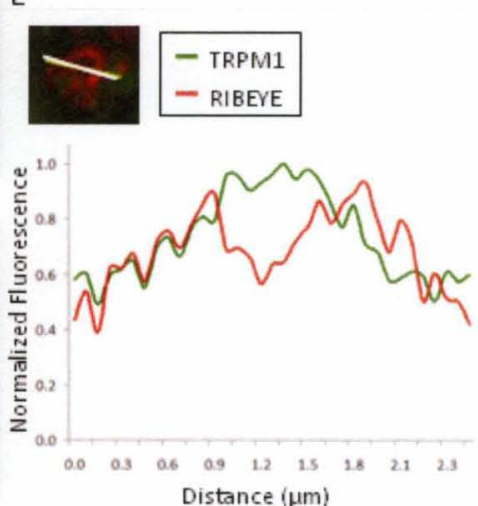

Figure 14: TRPM1 channel expression in murine retina. (A) Immunohistochemistry of transverse retinal sections in wildtype mice using a TRPM1 antibody. Expression is seen in the OPL (arrows) as well as on BC somas (arrowhead). Scale Bar is $20 \mu \mathrm{m}$ (B) A $2 \mu \mathrm{m}$ line (white bar) was drawn across the PNA-positive synapses. Fluorescence intensity for TRPM1 (green) and PNA (red) was determined and normalized to the peak value. (C) Whole mount retina showing co-localization of TRPM1 (green) and PNA (red). Scale bar is $5 \mu \mathrm{m}$. D. TRPM1 (green) expression in the OPL is closely aligned with RIBEYE (red). Scale bar is $5 \mu \mathrm{m}$. (E) A $2.5 \mu \mathrm{m}$ line was drawn through the RIBEYE-positive synapses. Fluorescence intensity for TRPM1 and RIBEYE was analyzed and normalized to the peak. Data show TRPM1 expression in murine retina is postsynaptic. 
Nyctalopin has been shown to be critical for DBC function (Gregg et al., 2003; 2007). However, there is no antibody to nyctalopin in mouse retina. As a consequence 1 used a transgenic mouse line in which a functional YFP-nyctalopin fusion protein was expressed in the DBC dendritic tips (Gregg et al., 2007). GFP antibodies were used to examine the localization of nyctalopin relative to TRPM1. Figure 15 shows the expression of TRPM1 and YFP-nyctalopin. As demonstrated previously, YFP-nyctalopin is restricted to dendritic terminals of DBCs in the OPL in murine retina (Gregg et al., 2007). Figure 15A shows that TRPM1 expression co-localizes with the nyctalopin puncta in the OPL. In addition, TRPM1 also stains BC somas within the ONL (Figure 15A). Higher magnification images of the OPL shows staining of TRPM1 and YFPnyctalopin consistent with the terminals of rods, small puncta, and larger cone DBC dendrites (Figure 15B). In the whole mount preparation, the large clusters of puncta are DBC dendrites on cone terminals and single puncta represent rod terminals (Figure $15 \mathrm{C}$ ). These data suggest that TRPM1 is localized on the dendritic tips of DBCs in close association with YFP-nyctalopin. 
A
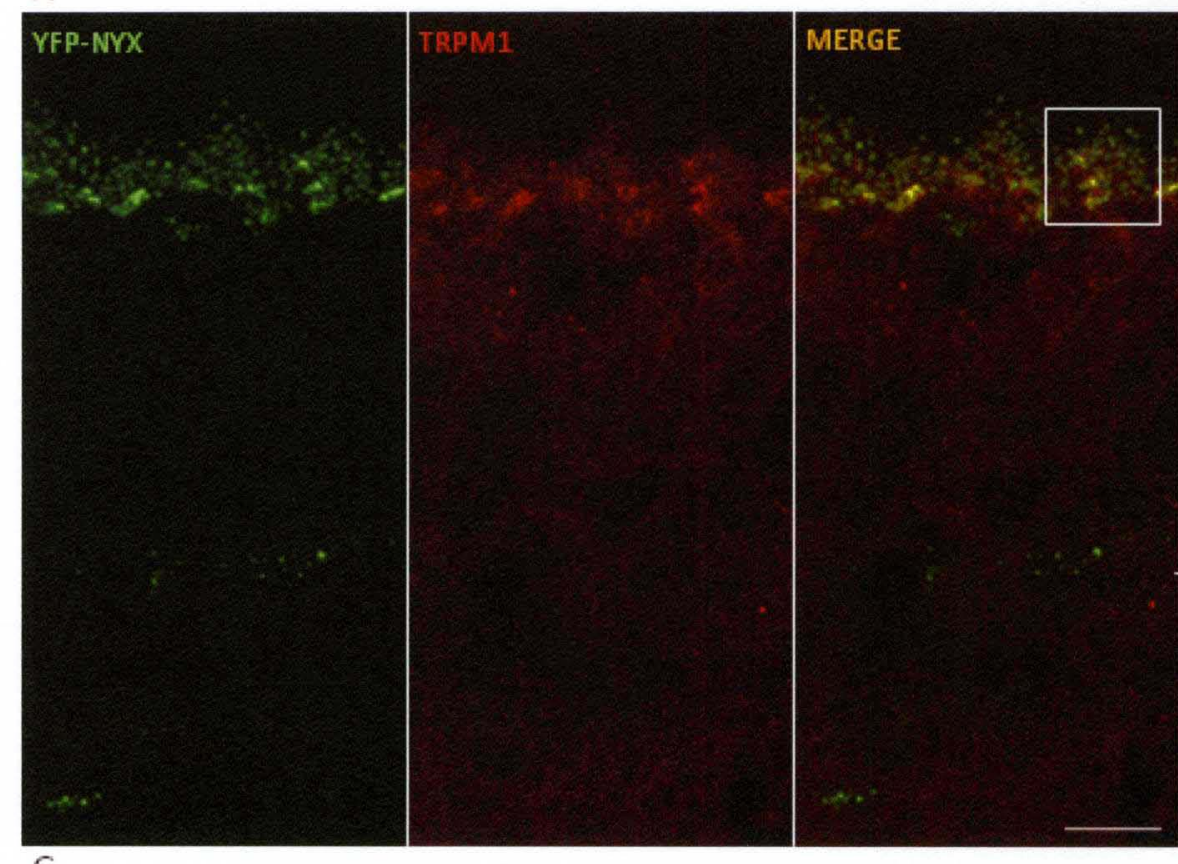

B
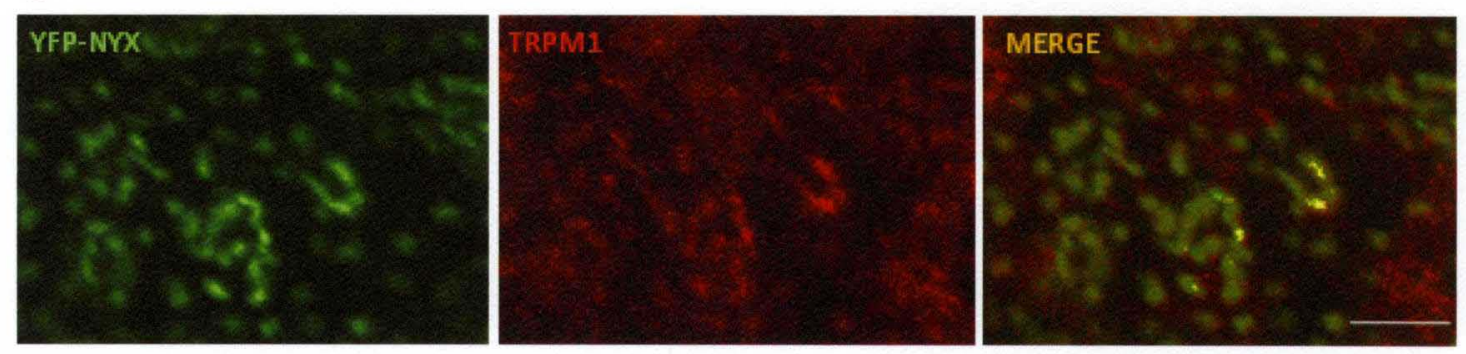

Figure 15: The TRPM1 channel co-localizes with nyctalopin in DBC dendrites. (A) Immunohistochemistry of retinal sections in TgYFPNyx mice using a TRPM1 antibody show expression in the OPL co-localizes with the YFP-nyctalopin fusion protein. Scale Bar is $20 \mu \mathrm{m}$. (B) A selected region of the OPL has been enlarged for closer analysis. (C) Images from whole mount TgYFPNyx retina also show co-localization of TRPM1 and YFP-nyctalopin in the OPL. Scale bar is $5 \mu \mathrm{m}$. This indicates the TRPM1 channel is localized to the DBC dendrites in the retina. 


\section{Discussion}

Research over the last several decades had pieced together important physiological and functional aspects of the DBC transduction channel; however, its identity remained unknown until 2008-2009. My dissertation work played a role in identifying the transduction channel as TRPM1.

I showed that full length TRPM1 could be amplified and cloned from murine retina CDNA. These experiments identified five retinal TRPM1 splice variants in mouse retina. Other TRPM family members also have multiple splice variants, but little is known about the functional roles. Some might act as either alternative isoforms of the channel or function in combination with full-length versions with modified properties. Recently, two novel human TRPM1 splice variants were identified from mRNA of primary human neonatal epidermal melanocytes (Oancea et al., 2009). These novel TRPM1 variants contained all six transmembrane domains and when expressed in SK-Mel19 melanoma cells generated an ionic current similar to endogenous TRPM1 current. These results suggest that the TRPM1 isoforms could form novel channels that conduct current and are biologically relevant.

An interesting aspect of the TPRM1 variants identified in melanoma cells was that each variant had a different expression pattern in melanocytes, retina and brain (Oancea et al., 2009). Expression of specific TRPM1 variants in the retina may be critical to DBC physiology. My studies of the developmental gene expression of TRPM1 isoforms determined that TRPM1, TRPM1-LF and TRPM1-N are likely expressed in DBCs. These variants were amplified by the qRT-PCR TRPM1-LF assay and had an expression profile consistent with those of other genes expressed in BCs (Kim et al., 2008). The TRPM1, TRPM1-LF and TRPM1-N variants contain the characteristic 6 transmembrane domains of TRP channels. On the other hand, the TRPM1-SV assay amplified only the TRPM1-SV variant, which is missing transmembrane domain 2 (S2). This splice variant 
displayed a temporal gene expression profile that is similar to genes expressed in PRs. Overall, the transcript profile of the TRPM1-LF and TRPM1-SV assays was consistent with the hypothesis that only the TRPM1 variants containing all six transmembrane domains would be expressed in the BCs.

Expression of TRPM1, TRPM1-LF and TRPM1-N variants could have a physiological role in BCs. TRPM1 is the full-length version, while the TRPM1-LF and TRPM1-N isoforms contain short intronic insertions (Table 2). Though small, these insertions could have drastic consequences on channel function. For example the TRPM1-LF variant has 6 amino acids (RKTRIH) inserted between transmembrane domains S4 and S5. The addition of 3 positive residues ( 2 arginines and 1 lysine) in the S4-S5 linker region could potentially impact TRPM1 channel function. Positive residues in the $\$ 4$ domain and S4-S5 linker form the gating charge in the TRPM8 channel as well as in Shaker K+ channels (Aggarwal \& MacKinnon, 1996; Seoh et al., 1996; Voet et al., 2007). The gating charge is part of the voltage-sensor. When the channel is closed the gating charge is positioned near the intracellular surface and then moves into the membrane when the channel opens (Jiang et al., 2003). Mutating the positive arginines to neutral alanines in this region resulted in reduced TRPM8 gating charge (Voets et al., 2007). Fulllength TRPM1 does not display voltage dependent properties; however, the additional positive residues in the TRPM1-LF isoform may lead to voltage sensing capabilities.

The TRPM1-N isoform has an additional 9 amino acids (VSIPGGRVE) inserted in the TRPM homology domain (MHD). The MHD is found in all TRPMs members and consists of four regions of sequence homology just downstream of the $\mathrm{N}$-terminus. Though the sequence is similar between the four regions they do not represent any known structural motifs. The MHD is suggested to play a role in multimeric assembly and/or normal surface trafficking of assembled multimeric channels, though the exact function remains to be established (Perraud et al., 2003, 
2004). Insertion of additional residues in the MHD region may affect the multimeric interactions between TRPM1 isoforms and could lead to novel TRPM1 heterotetrameic combinations.

Though sequence analysis of the two $\mathrm{BC}$ splice variants raises many possibilities, the exact function of these TRPM1 isoforms in the retina remains to be determined. Future studies on the TRPM1 variants could include: in situ hybridization analysis of the splice variants in tissue to confirm the expression pattern observed by qRT-PCR, generating antibodies to determine protein expression of the different TRPM1 variants and/or recapitulating each TRPM1 variant in a heterologous system and using patch clamp or calcium imaging to determine if they form a functional cation channel.

Transcriptional regulation and post-transcriptional mRNA processing are hardly predictable from genomic information. Two major aspects have to be considered: First, comparative analysis of the variants can help us understand structure-function relationships of TRPM1 proteins. Second, TRPM1 variants have been shown to be differentially expressed and might serve particular functions in different cell types (Oancea et al., 2009). Thus, to understand the physiological role and the molecular identity of native TRPM1 channels it is necessary to determine the subset of TRPM1 variants expressed in a specific kind of cell. In addition, though I identified some TRPM1 retinal variants there are presumably more; however, whether they form functional channels remains to be determined.

In $N y x^{\text {nob }}$ mice, DBC holding currents have decreased variance suggesting the TRPM1 channel is closed or absent from the membrane (Gregg et al., 2007). Interestingly, TRPM1 mRNA levels are reduced in $N y x^{n o b}$ mice compared to $C 57 B L / 6 J$ mice. This reduction was observed only in late developmental time points; however, was not shown to be statistically significant. Whether the loss of nyctalopin is directly causes a reduction of TRPM1 transcripts remains to be determined. PR genes, such as those encoding calcium channels, also have decreased mRNA 
expression in the $N y x^{n o b}$ mice (Bojang \& Gregg, 2008). These results suggest the altered activity in the $\mathrm{Nyx}^{\text {nob }}$ mice cause global decreases in mRNA transcription. However, the BC gene, Grm6, showed a 4-fold increase in mRNA levels at P13 (Bojang \& Gregg, 2008), so the mRNA reduction of TRPM1 may be a direct consequence of lack of nyctalopin.

Analysis of the temporal mRNA expression of TRPM1 and comparison with other genes expressed in BCs suggests it is expressed in BCs. This result was confirmed by immunohistochemistry using a commercial TRPM1 antibody. TRPM1 expression in human and murine retina is localized to the OPL and to BC somas in the INL. TRPM1 in the OPL was assessed by co-expression with the markers, RIBEYE or PNA, which showed expression was consistent with the DBC dendritic tips. The TRPM1 expression pattern in the retinal DBCs supports the ERG data that shows TRPM1 mutations in both humans and mice cause disrupted signal transmission from PRs to DBCs (Shen et al., 2009; Morgans et al., 2009; van Genderen et al., 2009; Aldo et al., 2009; Li et al., 2009).

In addition, my studies show that at the dendritic tips of DBCs, TRPM1 expression colocalizes with YFP-nyctalopin. Previously, YFP-nyctalopin was reported to co-localize with Grm6 and the absence of nyctalopin specifically prevented glutamate responses in DBCs (Gregg et al., 2007). The molecular function of nyctalopin is yet to be established, so co-localization with TRPM1 is intriguing. In my next chapter I examine whether the membrane proteins, nyctalopin, Grm6, and TRPM1, are required for each others expression at the DBC dendritic tips. This approach helps to elucidate their structural and functional interactions in vivo. 


\section{CHAPTER III: DETERMINE THE INTERDEPENDENCE OF NYCTALOPIN, TRPM1 AND GRM6 EXPRESSION IN DEPOLARIZING BIPOLAR CELLS IN THE MURINE RETINA}

Hypothesis Nyctalopin and TRPM1 expression in DBC dendrites is interdependent, while Grm6 expression is independent of either nyctalopin or TRPM1.

Introduction

The Grm6 signaling cascade is essential for light responses in retinal DBCs. Its disruption results in a loss of DBC mediated signaling, which can be characterized by using the electroretinogram (ERG) (see review by McCall \& Gregg, 2008). Congenital stationary night blindness type 1 (CSNB1) can be caused by mutations in GRM6, TRPM1 or NYX, which result in a no b-wave ERG phenotype and in $N y x^{n o b} B C$ in a lack of glutamate mediated depolarization (Pardue et al., 2003; Maddox et al., 2008; Pinto et al., 2007; Masu et al., 1995; Shen et al., 2009). Grm6 monitors changes in the concentration of synaptic glutamate released from PR terminals and relays that information via an intracellular G-protein cascade to regulate TRPM1 channels. TRPM1 is the nonselective cation channel that generates the DBC voltage response to light onset (Bellone et al., 2008, Shen et al., 2009, Morgans et al., 2009, Koike et al., 2009). Nyctalopin is an extracellular proteoglycan, and how it disrupts DBC signaling remains unknown (Pardue et al., 1998, Gregg et al., 2007).

The $N y x^{\text {nob }}$ mouse contains an $85 b p$ deletion in the $N y x$ gene, which encodes nyctalopin (Gregg et al. 2003). In the absence of nyctalopin, Grm6 and its second messenger, $\mathrm{G}_{0}$, are properly localized to DBCs dendrites (Ball et al., 2003); however, glutamate application to DBC dendrites does not mediate gating of TRPM1 through the Grm6 cascade (Gregg et al., 2007). 
Analysis of $N y x^{n o b}$ DBC activity using patch clamp recordings suggests that the TRPM1 channel is closed or absent (Gregg et al., 2007). These results imply that nyctalopin could be required for expression of TRPM1 in the membrane or for regulation of TRPM1 by the Grm6 cascade. Nyctalopin is predicted to be an extracellular protein, so it should only directly act on membrane-bound protein such as TRPM1, Grm6 or an unknown adaptor protein.

Previous studies were performed to determine how mutations of Grm6 and TRPM1 affect protein expression and localization. Expression of Grm6 CSNB-associated mutants in HEK293T cells leads to retention of the mutant Grm6 protein in the ER (Zeitz et al., 2007). The mutant protein was not trafficked to the plasma membrane, but when isolated and separated on a non-denaturing gel exhibited normal dimerization compared to wildtype (Zeitz et al., 2007). These data suggest that Grm6 CSNB mutations did not affect the overall structure of the protein, but did altered its localization.

Defects in trafficking of TRPM1 CSNB-associated mutants have been reported (Nakamura et al., 2010). In CSNB patients two TRPM1 gene mutations were identified in amino acids that are evolutionarily conserved, R624C (c.1870C >T) and F1075S (c.3224T>C). When these mutant channels were transiently expressed in the retina they failed to be transported to the tips of the DBC dendrites (Nakamura et al., 2010). Since these amino acids are highly conserved it suggests that they are most likely essential to the physiologic functions of TRPM1 channels. Overall, these results show that trafficking of Grm6 and TRPM1 to the plasma membrane is critical to generating normal DBC responses.

Membrane receptors and channels often form large complexes with multiple accessory proteins. These macromolecular complexes can be required for proper trafficking, assembly and stability of the receptor and/or channel. The first evidence for a TRP signaling complex (signalplex) comes from studies of Drosophila photoreceptor cells, called rhabdomeres. TRP, the 
founding member of the TRP family, is required for phototransduction in Drosophila (Cosens \& Manning, 1969). A central player in the TRP signalplex is Inactivation No After Potential D (INAD), a scaffold protein comprised of five PDZ protein interaction domains. The TRP signalplex includes core proteins that are constitutively bound to INAD consisting of TRP, PLC and PKC. The signalplex also contains non-core proteins that are not constitutively bound to INAD and include TRPL, rhodopsin, calmodulin, myosin III and the immunophilin FKBP59 (Chevesich et al. 1997; Xu et al. 1998; Wes et al. 1999). Unlike the core proteins, non-core proteins differ from the concentration of INAD and are believed to interact transiently with INAD. The TRP signalpex is required to retain TRP in the rhabdomeres and mediate rapid termination of the photoresponse (Chevesich et al. 1997; Adamski et al. 1998; Wes et al. 1999). Loss of one member of a macromolecular complex can have a deleterious effect on the entire complex.

In DBCs, there are several proteins that form a signalplex. G-protein cascades depend on several proteins including RGS (regulator of G protein signaling) and RGS9-1 anchor protein (R9AP). These proteins have been extensively studied in PRs. Recent studies have extended to DBCs and suggests that RGS11, GB5S and R9AP form a heterotrimer, which increases the rate of termination of the Grm6 cascade by facilitating GTP hydrolysis to GDP on the Ga $\alpha_{0}$ subunit (Chen et al., 2003; Morgans et al., 2007; Cao et al., 2008, 2009).

RGS proteins, such as RGS11, RGS9 and RGS7, contain three functional domains, the Dishevelled, EGL-10, Pleckstrin/DEP helical extension (DEP/DHEX), G-protein gamma subunit-like (GGL) and RGS, and form complexes with two binding partners R9AP and GB5S. The N-terminal DEP (Disheveled, EGL-10, Pleckstrin) and DHEX (DEP helical extension) domain form a single functional domain that mediates binding to the membrane anchor R9AP (Anderson et al., 2007; Cheever et al., 2008; Martemyanov et al., 2003; Narayanan et al., 2007). The central GGL domain is structurally similar to the conventional $\gamma$-subunits of G-proteins and forms a complex 
with GB5 (Snow et al., 1998; Witherow et al., 2000; Makino et al., 1999; Cabrera et al., 1998). The crystal structure of the RGS9-G 35 complex revealed that their interaction has the same orientation and association as those observed in the G $\beta \gamma$ complex (Cheever et al., 2008). At the C-terminus is the RGS domain that mediates conversion of active Ga-GTP species into inactive Ga-GDP species (Martemyanov \& Arshavsky, 2004). In addition, the crystal structure of the RGS domain of RGS7 shows direct contacts with the switch region of the activated Ga subunit to stabilize it in the transition state of GTP hydrolysis (Slep et al., 2001; Soundararajan et al., 2008).

Though the mechanism used to gate the TRPM1 channel in DBCs remains poorly understood, regulation through $\mathrm{Ga}_{0}$ is required (Dhingra et al., 2000, 2002). Therefore, the kinetics of the $D B C$ response depend on how quickly $G a_{0}$ inactivates. RGS proteins control the timing of this inactivation, so play a critical role in shaping the magnitude and duration of the DBC response. For a complete review on RGS proteins see Anderson et al (2009).

Disrupting one of the proteins in the RGS11, GB5S and R9AP heterotrimer, such as in G65 mice, results in loss of the other two proteins (Cao et al., 2008, 2009). In addition, stability of the RGS11 protein requires association not only with GB5S and R9AP, but also with Grm6. In Grm6 $6^{\text {nob4 }}$ mice, immunohistochemistry showed a lack of expression of RGS11, GB5S and R9AP in the DBC dendrites (Cao et al., 2009). Total protein levels in $\mathrm{Grm} 6^{\text {nob4 }}$ mice were maintained for R9AP and GB5S, though RGS11 levels were significantly reduced. These results suggest G 35 , R9AP and Grm6 interact with RGS11, which protects RGS11 from proteolytic degradation (Cao et al., 2009). Grm6 interaction with RGS11 was confirmed in a heterologous expression system (Cao et al., 2009). These results clearly show how individual components of macromolecular complexes depend on other complex members for proper localization.

In this chapter, I investigate the interdependence of nyctalopin, Grm6 and TRPM1. All three proteins are expressed on the dendritic tips of DBCs (Gregg et al., 2007; Morgans et al., 
2009; Shen et al., 2009). I expect that protein complexes in the DBC dendrites will require protein-protein interactions to maintain stability; therefore, the loss of one member of the complex may lead to mislocalization or proteolytic degradation of the other members. Additionally, the expression requirements for each membrane protein may provide insight into their in vivo interactions and help to determine the function of nyctalopin.

\section{Results}

Confocal microscopy shows that nyctalopin co-localizes with Grm6 and TRPM1 at the tips of the DBC dendrites (Figure 16, Gregg et al., 2007; Morgans et al., 2009; Pearring et al., 2010). TgYFPNYX mice express a YFP-nyctalopin fusion protein in the DBC dendritic tips. Immunohistochemistry with a GFP antibody for YFP-nyctalopin shows it co-localizes with both TRPM1 (Figure 16A) and Grm6 (Figure 16B). In addition, electron microscopy has shown that Grm6 and nyctalopin are restricted to the DBC dendritic tips (Gregg et al., 2007). Since all three of these proteins have a similar expression pattern and are critical to the G-protein transduction cascade in DBCs I expect they will be closely associated and their expression may be interdependent. 

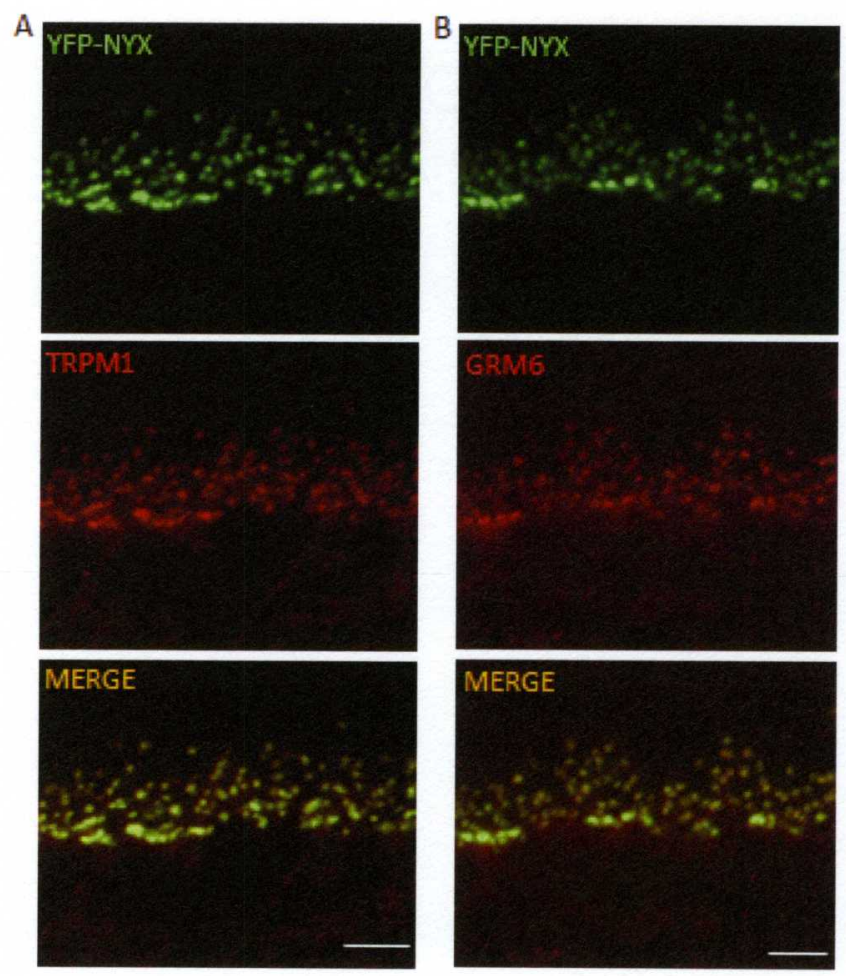

Figure 16: Expression of nyctalopin in TgYFPNYX mice. (A) Immunohistochemistry of YFP-nyctalopin (green) with TRPM1 (red). TgYFPNYX retina shows YFP-nyctalopin co-localizes with the TRPM1 in the OPL (yellow, merge). Scale bar is $5 \mu \mathrm{m}$. (B) YFP-nyctalopin (green) expression shown with double labeling with the postsynaptic marker, Grm6 (red). YFP-nyctalopin is co-localized with Grm6 in DBC dendrites (yellow, merge). Scale bar is $5 \mu \mathrm{m}$.

\section{TRPM1 localization in the absence of nyctalopin}

To examine the interdependence of nyctalopin and TRPM1, I determined if TRPM1 expression is altered in $\mathrm{Nyx}^{\text {nob }}$ retina. TRPM1 expression in the absence of nyctalopin was determined using immunohisochemistry in $\mathrm{Nyx}^{\text {nob }}$ retina transverse sections and in whole mount preparations. In $C 57 B / / 6 J$ retina, TRPM1 is expressed in the dendritic tips of DBCs where it overlaps with the cone pedicle marker, PNA (yellow, arrows in merge, Figure 17A), as well as in the somas of the INL (arrow heads, Figure 17A). In the absence of nyctalopin, TRPM1 is no longer on the DBC dendritic tips (Figure 17A). Reduced intensity of TRPM1 staining in the OPL is obvious when TRPM1 is merged with PNA, which remains red (Figure 17A). In addition, this can 
be seen by an absence of TRPM1 puncta in the OPL of whole mount preparations of Nyx ${ }^{\text {nob }}$ retina (Figure 17B). The decrease in TRPM1 expression in the OPL of $N y x^{\text {nob }}$ retina is similar to images from $T R P M 1 \%$ retina (Figure 17B, Morgans et al., 2009; Koike et al., 2009).

To further examine the reduction in TRPM1 expression in the OPL of $N y x^{\text {nob }}$ retina, I quantified the intensity of TRPM1 pixels to PNA pixels (see Methods for details). These data show that in $\mathrm{C} 57 \mathrm{BI} / 6 \mathrm{~J}$ mice the peak intensity for TRPM1 overlaps with the peak for PNA, though TRPM1 intensity is not equal to PNA (Figure 18A). The difference in peak intensity between the two channels is likely due to the quality of the TRPM1 \#92 antibody. As described in Chapter II, this antibody was made to a human TRPM1 peptide that only has $57.5 \%$ homology to murine TRPM1. In Nyx ${ }^{\text {nob }}$ mice, the TRPM1 intensity was dramatically reduced and no TRPM1 staining was observed within the PNA-positive puncta (Figure 18B). These results suggest that localization of TRPM1 to the DBC dendritic tips requires expression of nyctalopin. 


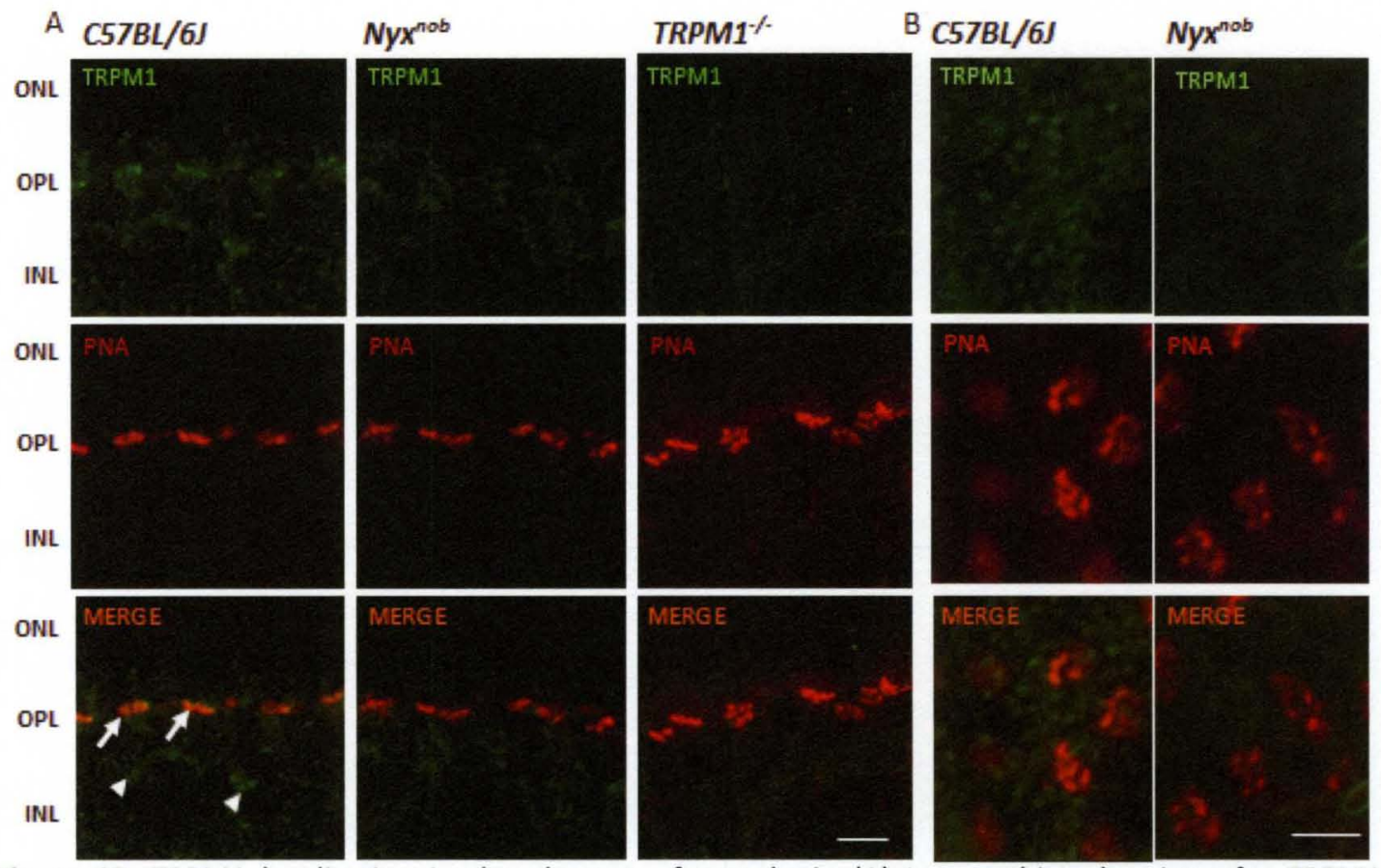

Figure 17. TRPM1 localization in the absence of nyctalopin (A) Immunohistochemistry for TRPM1 (green) in $\mathrm{C} 57 \mathrm{Bl} / 6 \mathrm{6}, \mathrm{Ny} \mathrm{x}^{\mathrm{nob}}$ and TRPM1 ${ }^{\%}$ transverse retinal sections. PNA (red) was used to stain the cone terminals in the OPL that co-localize with TRPM1 in C57BI/6J mice (yellow, arrows Merge). TRPM1 is also present in the INL (arrow heads). TRPM1 channel expression is absent from the DBC terminals in $\mathrm{Nyx}^{\text {nob }}$ mice (red, Merge), which is similar to TRPM1 $1^{-1}$ mice (red, Merge). (B) Horizontal sections show TRPM1 (green) puncta co-localize with PNA (red) staining in the OPL of $C 57 \mathrm{~B} / / 6 \mathrm{~J}$ retina. The TRPM1 expression in the OPL is absent in $N y x^{n o b}$ sections. Scale bars are $5 \mu \mathrm{m}$. 

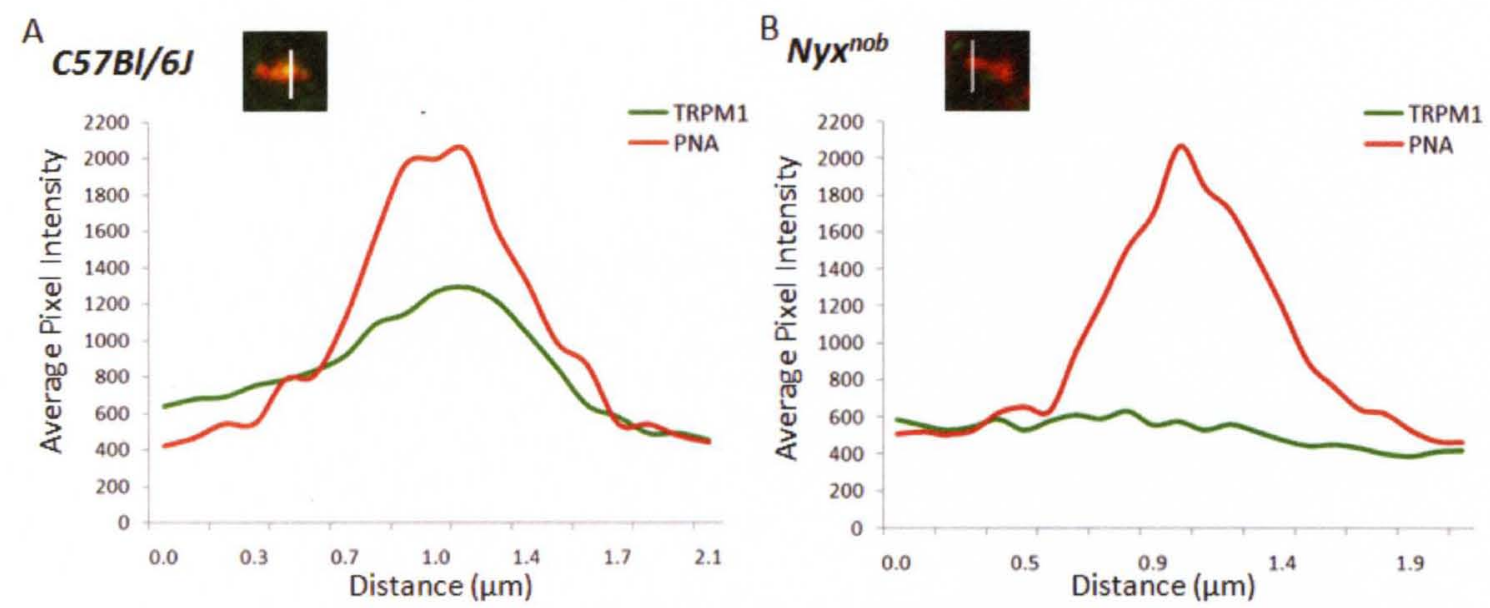

Figure 18. Quantitative analysis of the TRPM1 fluorescence in C57BI/6J and $N y x^{n o b}$ mice. (A) Analysis of TRPM1 fluorescent intensity in the OPL of $C 57 B / / 6 J$ retinal sections. A line of $2 \mu \mathrm{m}$ (white bar) was drawn through the center of distinct PNA-positive synapses. The fluorescence intensity along this line was scanned to generate profiles corresponding to PNA (red) and TRPM1 (green). (B) In Ny nob retinal sections TRPM1 pixel intensity is not above background within the PNA-positive punctate. Shown traces are an average from two images with 10 intensity scans for each image.

\section{Nyctalopin expression in the absence of TRPM1}

TRPM1 requires nyctalopin for expression in the OPL. The next step was to determine if this dependence was interchangeable and I examined nyctalopin expression in the absence of TRPM1 using TRPM1-/- mice.

To detect nyctalopin expression I crossed TgYFPNYX mice onto the TRPM1 ${ }^{-1}$ background to generate TgYFPNYX/TRPM1 ${ }^{-}$mice. Transverse sections and whole mounts were prepared for $\operatorname{TgYFPNYX/TRPM1}{ }^{-}$and control (TgYFPNYX) retinas. Figure 19 shows the expression pattern of YFP-nyctalopin stained with a GFP antibody and TRPM1. YFP-nyctalopin is present as puncta located on the tips of rod $\mathrm{BC}$ dendritic terminals as well as adjacent to cone terminals in control and TRPM $^{\%}$ retinas (Figure 19A). In transverse sections from $\operatorname{Tg}$ YFPNYX/TRPM1 ${ }^{-}$mice, YFPnyctalopin expression in the OPL is indistinguishable from the control sections. This finding is also apparent in the confocal optical sections at the level of the OPL obtained from whole mounts (Figure 19B). 
To further refine the localization of nyctalopin in the absence of TRPM1, I examined nyctalopin's expression relative to PNA, Grm6 and RIBEYE. Grm6 is located at the dendritic tips of DBCs and has been shown to be co-localized with nyctalopin (Masu et al., 1995; Vardi \& Morigiwa, 1997; Gregg et al., 2007). The staining pattern for YFP-nyctalopin expression relative to Grm6 was indistinguishable between $\operatorname{TgYFPNYX}$ and $\operatorname{Tg} Y F P N Y X / T R P M 1^{-1}$ retinal sections (Figure 20A). Grm6 is expressed at the tips of the DBCs were it co-localizes with YFP-nyctalopin (Gregg et al., 2007). The post-synaptic localization of nyctalopin in the OPL was confirmed by expression relative to RIBEYE (red, Figure 20B) or PNA (red, Figure20C). YFP-nyctalopin did not localize with the pre-synaptic marker, RIBEYE, but co-localized with the cone pedicle marker, PNA. These results reveal that nyctalopin expression and localization to the dendritic tips of DBCs is independent of TRPM1 expression.

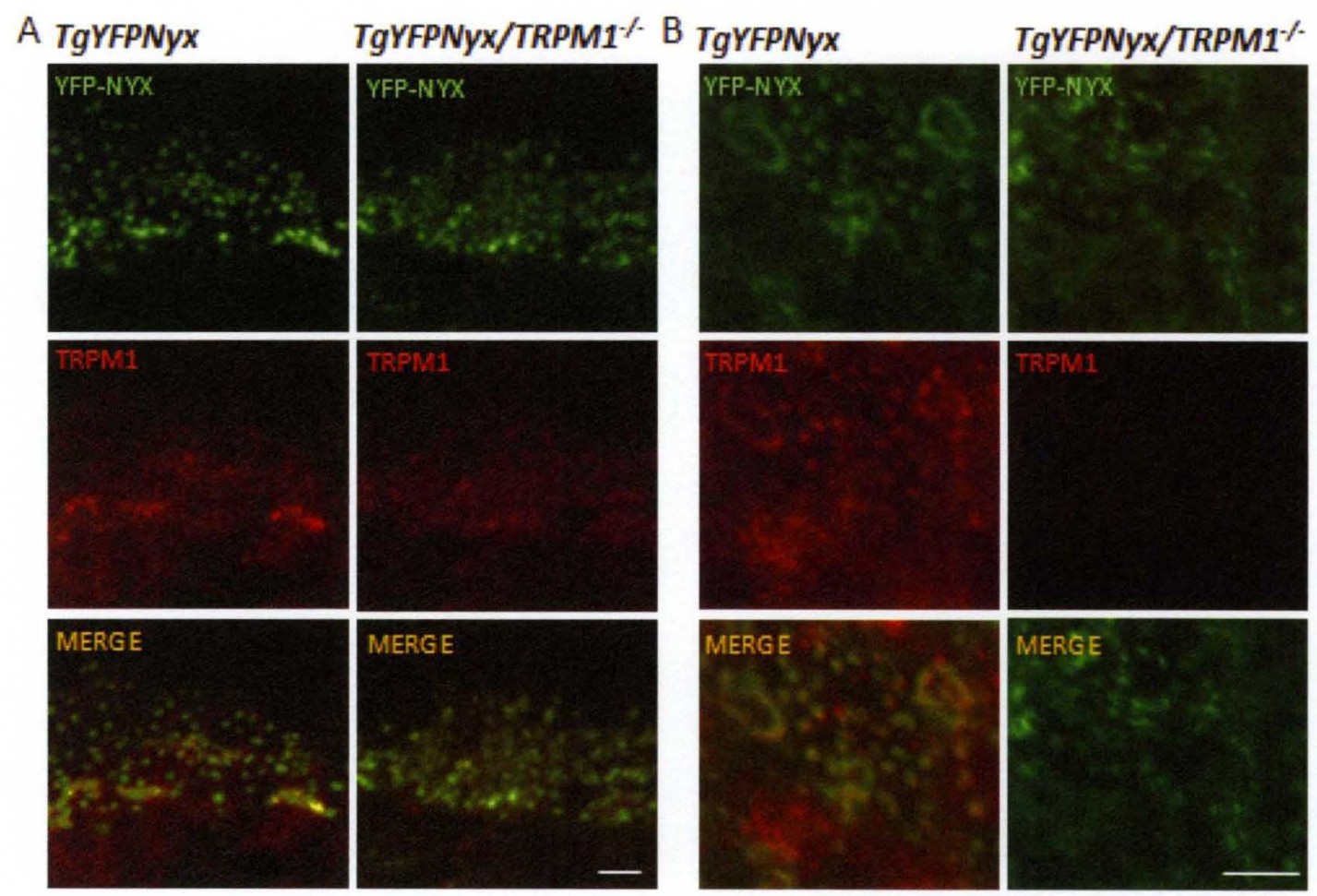

Figure 19: Nyctalopin expression in the absence of TRPM1. (A) In TgYFPNYX mice, YFP-nyctalopin (green) is co-localized with TRPM1 (red) as seen by the yellow in the merged image. In the TgYFPNYX/TRPM $1^{-1}$ mice, there is no staining for TRPM1, but YFP-nyctalopin is normally expressed. Scale bar is $5 \mu \mathrm{m}$. (B) Horizontal optical sections through the OPL from TgYFPNYX and TgYFPNYX/TRPM1 ${ }^{-1}$ whole mount preparations also show YFP-nyctalopin is localized to the OPL in the absence of TRPM1. Scale bar is $5 \mu \mathrm{m}$. 



\section{Nyctalopin expression pattern in the absence of Grm6}

I examined the localization of nyctalopin in the absence of Grm6. The $N y x^{\text {nob }}$ mouse has normal expression pattern of Grm6 at the dendritic tips of the DBCs. This indicates that Grm6 stability and targeting to the membrane is independent of nyctalopin expression (Ball et al., 2003). However, nyctalopin expression in a Grm6 mutant has not been determined.

Nyctalopin expression was examined in the absence of Grm6 expression by crossing TgYFPNYX mice onto the Grm6 $6^{\text {nob4 }}$ genetic background to generate $\operatorname{Tg} Y F P N Y X / G r m 6^{n o b 4}$ mice. Immunohistochemistry for YFP-nyctalopin using a GFP antibody was performed on sections from $\operatorname{TgYFPNYX/Grm6^{nob4}}$ retina. Figure $21 \mathrm{~A}$ shows the expression pattern of YFP-nyctalopin and Grm6 in sections from TgYFPNYX and TgYFPNYX/Grm6 ${ }^{\text {nob4 }}$ mice. In TgYFPNYX retinas, YFPnyctalopin was present at the tips of the DBC dendrites, where it co-localizes with Grm6 (Gregg et al., 2007). In the absence of Grm6, the staining pattern of YFP-nyctalopin appears indistinguishable from controls (Figure 21A).

To further examine the expression pattern of YFP-nyctalopin in Grm6 $6^{\text {nob4 }}$ mice, I double labeled with PNA, RIBEYE and PKC $\alpha$. PNA is a lectin that binds the inner and outer segments as well as the axon terminals of cone PRs. RIBEYE is a ribbon synapse protein that is expressed in the axon terminals of PRs and BCs. PKC $\alpha$ is a protein kinase that is specifically expressed in the cytosol of rod DBCs.

In retina sections from $\mathrm{Grm6}^{-1-}$ mice, YFP-nyctalopin co-localized with the PNA (Figure 21B); however, YFP-nyctalopin did not overlap with RIBEYE (Figure 21C). This staining suggests YFP-nyctalopin expression in the absence of Grm6 remains postsynaptic. PKC $\alpha$ (red) labels rod BC bodies in the INL along with their dendrites that project into the OPL (Haverkamp \& Wässle, 2000). Double labeling for YFP-nyctalopin and PKC $\alpha$ in $T g Y F P N Y X / G r m 6^{\text {nob4 }}$ showed that YFPnyctalopin is localized as puncta at the tips of rod BCs (arrowhead, Figure 21D). Large puncta 
representing staining of YFP-nyctalopin on cone terminals also can be seen at the inner part of the OPL (arrows, Figure 21D). This immunohistochemistry demonstrates that nyctalopin is properly restricted to the DBC dendritic tips in the absence of Grm6. Therefore, nyctalopin localization and stability is independent of Grm6. 
A
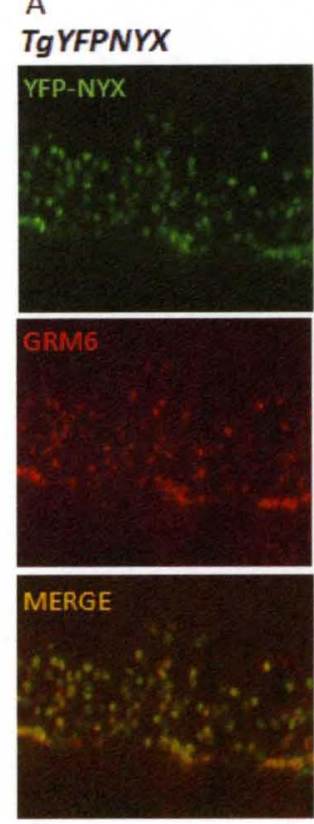

C

TgYFPNYX


B

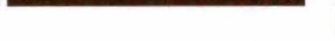

D
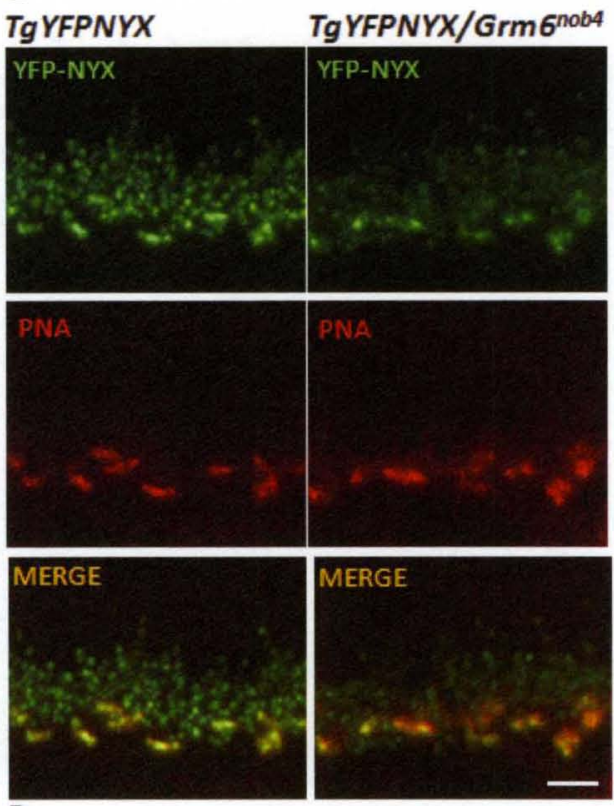

\section{TgYFPNYX}

TgYFPNYX/Grm6 $6^{\text {nob4 }}$
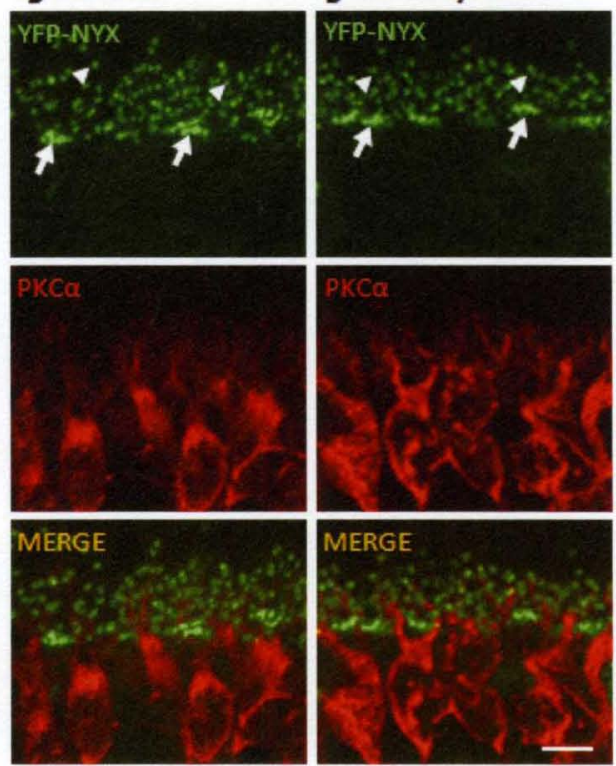

Figure 21: Nyctalopin expression in the absence of Grm6. (A) YFP-nyctalopin (green) staining in control, TgYFPNYX sections, co-localizes with Grm6 (red). In TgYFPNYX/Grm6 $6^{\text {nob4 }}$ sections, Grm6 staining is absent, but YFP-nyctalopin remains expressed. YFP-nyctalopin staining is indistinguishable between TgYFPNYX and TgYFPNYX/Grm $6^{\text {nob4 }}$ mice as shown by double-labeling with PNA (B) and RIBEYE (C). (D) Staining of YFP-nyctalopin (green) and rod BC marker, PKC $\alpha$ (red) shows nyctalopin puncta is localized to the tips of rod BCs (arrowheads). Larger nyctalopin puncta represent localization in cone BCs dendrites (arrows). Scale bar is $5 \mu \mathrm{m}$ for all images. 


\section{TRPM1 Localization in the Absence of Grm6}

Previous reports have shown that Grm6 localization to the DBC dendritic tips of DBCs is independent of TRPM1 (Morgans et al., 2009; Koike et al., 2009). To determine if TRPM1 expression depends on Grm6, I examined TRPM1 expression pattern in a Grm6 mutant mouse. Since the absence of Grm6 expression does not alter nyctalopin expression, YFP-nyctalopin was used as a marker for the DBC dendritic tips in retinal sections from control, TgYFPNYX, and mutant, TgYFPNYX/Grm6 ${ }^{\text {nob4 }}$. Figure 22A shows TRPM1 expression in the OPL and on DBC somas in the INL of TgYFPNYX retinas. The staining pattern in the OPL is punctate, which is typical of staining of DBC dendritic tips. TRPM1 co-localizes with the punctate staining pattern of YFPnyctalopin (yellow in merged Figure 22A). $\ln G r m 6^{\text {nob4 }}$ sections, the punctate staining for TRPM1 is reduced in the OPL although it remains in the INL (Figure 22B). This absence of TRPM1 from the OPL is apparent in the merged image with nyctalopin, where there is no evidence of colocalization (green, arrow Figure 22B). TRPM1 staining in the INL does not appear to be altered

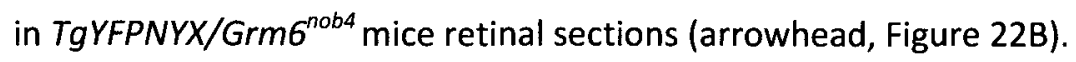

This reduction of TRPM1 expression at the DBC dendritic tips in $\mathrm{Grm} 6^{\text {nob4 }}$ sections was further examined by quantifying the pixel intensity of staining for TRPM1 to YFP-nyctalopin. In TgYFPNYX mice the TRPM1 peak lies within the YFP-nyctalopin peak intensity. In the TgYFPNYX/Grm6 $6^{\text {nob4 }}$ mice, there is no evidence of TRPM1 fluorescence in the average from 30 intensity profiles across nyctalopin-positive puncta (Figure $22 \mathrm{C}$ ). These results suggest TRPM1 expression in the OPL depends on Grm6 expression. 
A

\section{TgYFPNYX}
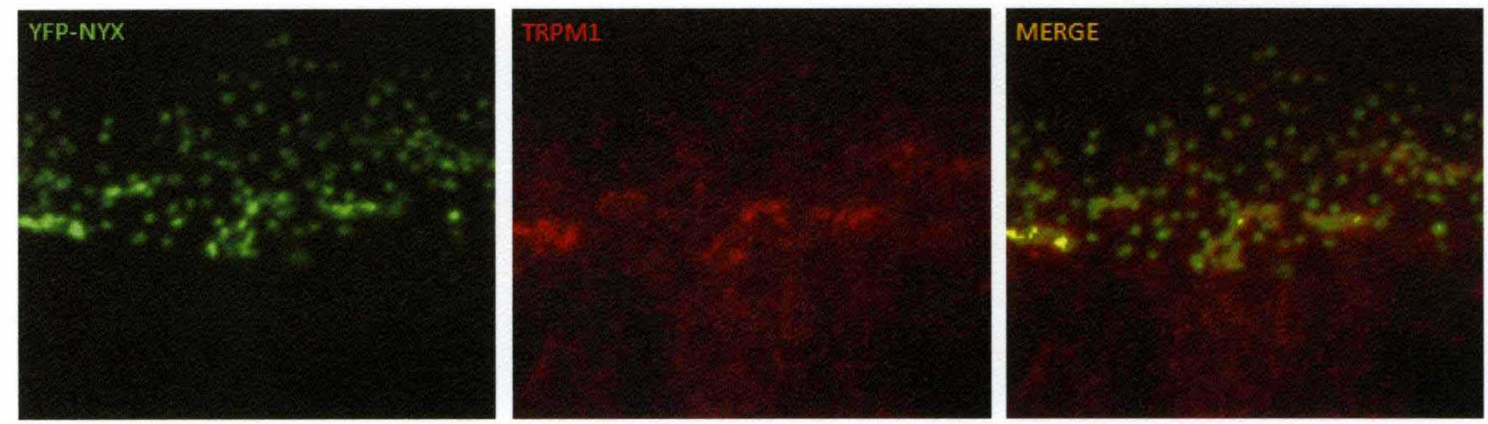

B

TgYFPNYX/Grm6 $6^{\text {nob4 }}$
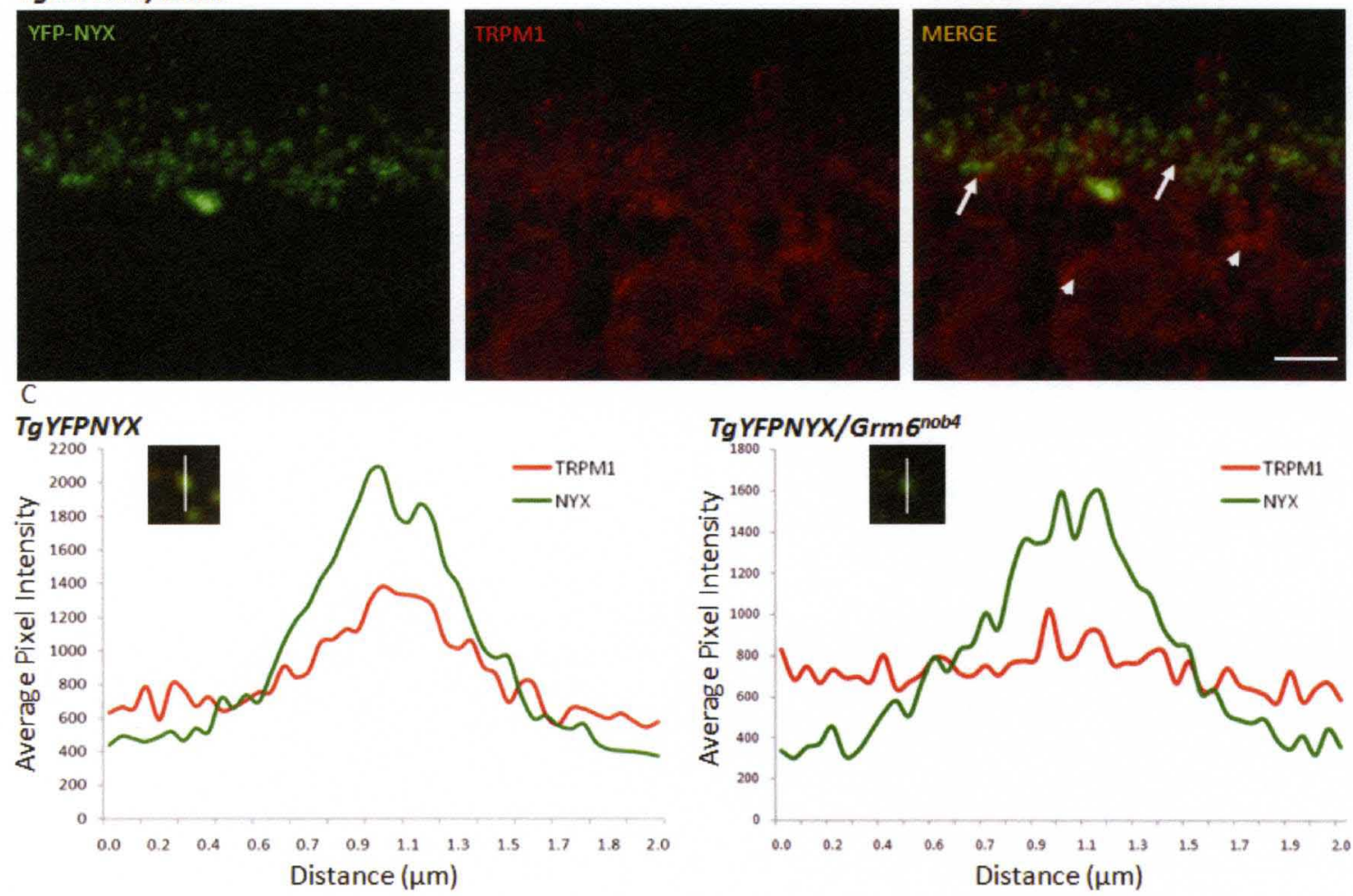

Figure 22: TRPM1 expression in the absence of Grm6. (A) Immunohistochemistry of YFP-nyctalopin (green) and TRPM1 (red) in TgYFPNYX retinal sections. YFP-nyctalopin co-localizes with the TRPM1 in the OPL (merge). (B) In TgYFPNYX/Grm6 ${ }^{\text {nob4 }}$ sections, the expression of nyctalopin is unaffected; however, TRPM1 expression is absent from the OPL (arrow). TRPM1 expressed in the INL is present in the absence of Grm6 (arrowhead). Scale bar is $5 \mu \mathrm{m}$ (C) Quantitative analysis of the TRPM1 and nyctalopin intensity. A line of $2 \mu \mathrm{m}$ (white bar) was drawn through the center of the distinct YFP-positive synapses. In TgYFPNYX, the distribution profiles of YFP-nyctalopin (green) and TRPM1 (red) correspond; however, TRPM1 pixel intensity no longer overlaps with nyctalopin puncta in the absence of Grm6. Shown traces are an average from three images with 10 intensity scans for each image. 
There are three mutant alleles of Grm6: $G r m 6^{n o b 4}, G r m 6^{n o b 3}$ and $G r m 6^{\prime}$. To confirm that TRPM1 is reduced in the DBC dendrites in all of these Grm6 mutant mice, I performed immunohistochemistry for using the TRPM1 antibody on retinal sections from $\mathrm{Grm}^{\mathrm{nob3}}$ and $\mathrm{Grm}^{\prime}{ }^{\prime}$ mice. Figure 23 shows immunohistochemistry for TRPM1 and PNA in C57BI/6J, Grm $6^{\text {nob3 }}$ and $\mathrm{Grm}^{\prime}{ }^{\perp}$ transverse retinal sections. TRPM1 is expressed in the dendritic tips of the DBCs, and co-localizes with PNA in retinas from $C 57 B / / 6 J$ animals (yellow in merge, Figure $23 A$ ). However, in both the Grm6 mutants TRPM1 puncta are absent from the OPL although TRPM1 staining remains in the DBC soma. This is apparent in the merged images, which shows no evidence of co-localization (Figure 23A). The average pixel intensity in the green and red channels was determined by measuring the intensity across PNA-positive puncta (Figure 23B). Intensity peaks for the TRPM1 and PNA puncta overlap in the C57BI/6J OPL, but in the Grm6 mutants TRPM1 lacks a intensity peak within the PNA puncta. These results shows that regardless of the alteration in Grm6 (knockout or spontaneous mutation) that TRPM1 is not properly localized to the dendritic tips of DBCs. 


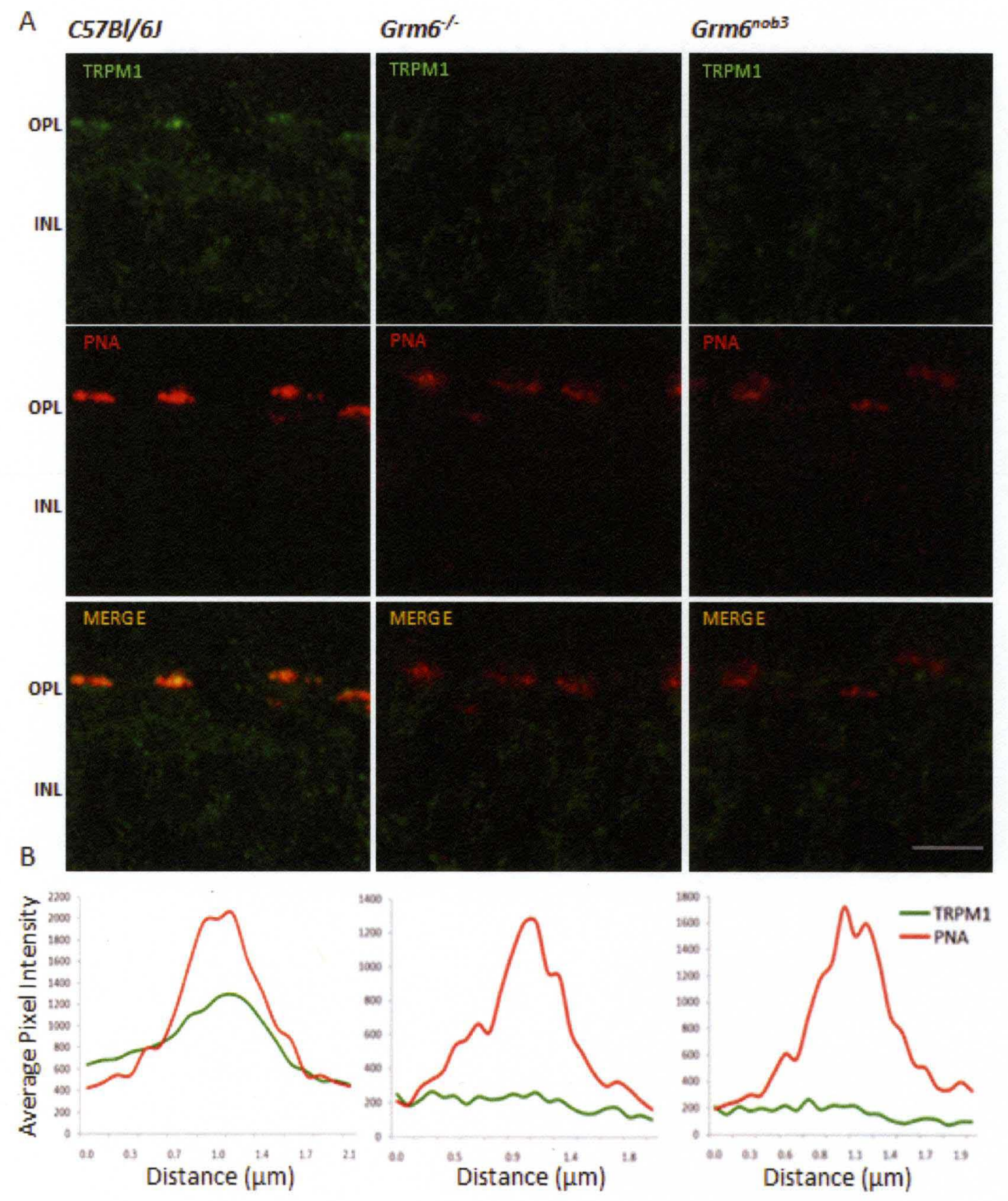

Figure 23: Immunohistochemistry of TRPM1 in Grm6 mutant mice. (A) C57BI/6J transverse retinal sections shows co-localization between TRPM1 (green) and PNA (red) in the OPL (yellow, merge). In $\mathrm{Grm}^{-/}$sections, expression of TRPM1 in the OPL is reduced. TRPM1 is no longer co-localized with PNA. The absence of TRPM1 from postsynaptic structures is also present in the Grm $6^{\text {nob3 }}$ mutants. Scale bar is $5 \mu \mathrm{m}$ (B) Quantification of the intensity of TRPM1 fluorescence across the PNA-positive puncta. TRPM1 intensity is co-localized with PNA in controls, C57BI/6J, but is absent from PNA puncta of Grm6 mutants. 


\section{Discussion}

Receptors and channels are known to form macromolecular complexes in the membrane and disruption of one member can lead to disruption of the entire complex. Grm6, nyctalopin and TRPM1 are three membrane proteins required for the normal DBC response to light. I used several mutant mouse models to determine how loss of one DBC membrane protein affected the expression of the other two proteins. As discussed in the introduction, interdependence of proteins has also been reported for the RGS, Gß5S, Grm6 and R9AP proteins in the DBCs (Cao et al., 2009).

Studies of DBC activity in $N y x^{n o b}$ mice suggest that without nyctalopin the TRPM1 channel is closed or absent (Gregg et al., 2007). My immunohistochemical results show TRPM1 expression is absent from the dendritic tips of DBCs in the $N y x^{\text {nob }}$ retina. Since the synaptic architecture of the OPL is normal in $N y x^{n o b}$ mice (Ball et al; Gregg et al., 2007) the reduction of TRPM1 implies nyctalopin is required for the stability or trafficking of TRPM1 in the DBC dendrites. The exact mechanism involved is unknown, but there are several possibilities.

Nyctalopin could play a role in maintaining TRPM1 integrity. Nyctalopin's LRR domain structure is similar to ankyrin repeats, which folds into two anti-parallel helices followed by a beta-hairpin (Mosavi et al., 2004; Sedgwick \& Smerdon 1999). TRPA, TRPN, TRPV and TRPC subfamilies contain ankyrin repeat domains in their N-terminus (Montell, 2005; Gaudet, 2008). Deletion of the ankyrin domain impairs TRP channel assembly and trafficking to the cell membrane (Jung et al., 2002; Chang et al., 2004; Erler et al., 2004). Nyctalopin could be acting as an auxiliary ankyrin domain for TRPM1 by supporting subunit assembly in the DBCs. When nyctalopin is absent the TRPM1 channel would not assemble and be unable to properly localize to the DBC dendrites. 
Nyctalopin could be playing a role in trafficking the TRPM1 channel to the plasma membrane. In neurons, some channels contain amino acid motifs that direct their axonal or dendritic targeting (Gu et al., 2003; Rivera et al., 2003; Kanaani et al., 2002). Nyctalopin could be acting as a target for TRPM1 channel expression to the membrane. TRPM1 in other cells, such as melanocytes, is predominately localized to the endoplasmic reticulum (Oancea et al., 2009). In fact, many TRP channels play an intracellular role (Oancea et al., 2006; Bezzerides et al., 2004). Perhaps, nyctalopin is required to transport the TRPM1 channel to the membrane, so it is at the plasma membrane only in cells that also express nyctalopin.

Interestingly, my results show that nyctalopin transport and stability is not dependent on either TRPM1 or Grm6 postsynaptic expression. I hypothesized that if nyctalopin formed a complex with these proteins it would be mis-localized or degraded in the absence of its interacting partner. Positive nyctalopin expression suggests it could be interacting with multiple proteins, so that loss of one does not affect the overall stability of all. Therefore, it would be critical to determine if nyctalopin localizes in DBC dendritic tips in the double knockout mouse, $\operatorname{TRPM} 1^{\%} / G r m 6^{n o b 4}$. Additionally, if nyctalopin is acting as an anchor to stabilize membrane proteins in the synaptic site its localization may not depend on expression of its partners. It may be acting solely as the glue that holds the complex together.

In contrast to my hypothesis that TRPM1 would only require nyctalopin for proper localization I found that Grm6 mutants showed reduced intensity of TRPM1 staining in the OPL. Elimination of Grm6 leads to the failure of the DBC to establish synaptic connections with the PR terminals (Cao et al., 2009). In addition, ectopic ribbon synapses in the DBC dendritic tips have been identified in Grm6 mutants (Ishii et al., 2009). These results suggest that signaling through Grm6 is required for proper synaptic connectivity of the DBCs. Therefore, loss of TRPM1 may be due to disruption of the DBC synaptic architecture, not through direct interaction with Grm6. 
Alternatively, TRP channels are reported to have activity dependent trafficking. For example, growth factor stimulation initiates rapid translocation of the TRPC5 channel from vesicles held in reserve near the plasma membrane (Bezzerides et al., 2004). Rapid translocation of TRPM1 channels into the postsynaptic membrane could be regulated by Grm6 activity. To provide for spatio-temporally restricted cation entry through TRPM1 channels, the channels may be sequestered into vesicles and inserted into the plasma membrane upon termination of the Grm6 cascade. The rapid incorporation of TRPM1 maintained in readily available vesicles, followed by Grm6 activated endocytosis would represent a secondary method to control membrane levels of TRPM1, possibly during light adaptation, since it is likely not fast enough to generate the millisecond response to glutamate. Light adaptation is the ability of PRs to respond to a large range of light intensities. Protein translocation has been observed for the G-protein signaling molecules present in PRs and contributes to light adaptation (for a complete review see Calvert et al., 2006).

Nyctalopin's role in the Grm6 cascade has remained elusive since it was first identified in 1998 (Bech-Henson et al., 1998, Pardue et al., 1998). My current results suggest nyctalopin is involved in transport or stability of TRPM1 in the dendritic tips of DBCs. To date, no SLRP protein has been implicated in trafficking or anchoring of a channel and this finding could be a novel mechanism for SLRP proteins. In the next chapter, I will investigate the biochemical interactions between Grm6, nyctalopin and TRPM1, which will shed light on the protein complex present in the DBCs. 


\section{CHAPTER IV. DETERMINE INTERACTIONS BETWEEN NYCTALOPIN, GRM6 AND/OR TRPM1}

Hypothesis Nyctalopin will interact with TRPM1, but not Grm6.

Introduction Nyctalopin is a 476 amino acid member of the small leucine-rich repeat proteoglycan family (SLRP). The SLRP family contains several extracellular matrix proteins that are composed of leucine-rich repeats (LRRs) flanked by two conserved cysteine-rich regions (McEwan et al., 2006). Nyctalopin contains an $\mathrm{N}$-terminal signal sequence, the LRR domain, a highly conserved linker region and a domain that attaches to the membrane. Human nyctalopin is predicated to use a glycosylphosphatidyl-inosital-anchor (GPI anchor) to attach to the membrane, while murine nyctalopin contains a putative transmembrane domain at the Cterminus (Bech-Hansen et al., 2000; Pusch et al., 2000; O'Conner et al., 2005). Nyctalopin is the only member of the SLRP family that is attached to the plasma membrane.

The LRR domain is believed to be the functional region of the SLRP proteins (Buchanan \& Gay, 1996; Kobe \& Kajava, 2001). LRR are tandem repeats that are found across functionally diverse proteins and are involved in a variety of cellular process such as cell adhesion, signal transduction, DNA repair and RNA processing (Kobe and Deisenhofer, 1994). One LRR corresponds to a $20-29$ amino acid sequence ( $\operatorname{xx} L x L x x N x L)$ that form a $\beta$-strand parallel to an $\alpha$ helix connected by loops (Hocking et al., 1998; lozzo, 1999). These tandemly repeated structures are arranged as a horseshoe-shaped molecule with a curved parallel $\beta$-sheet lining the concave side and the $\alpha$-helices forming its convex side (refer to Figure 5A). The LRR domain of nyctalopin is structurally distinct from the other SLRPs. It has 11 core LRRs that are flanked by an N- and C- 
terminal cysteine-rich LRR cap $\left(\mathrm{CX}_{3} \mathrm{CXCX}_{6} \mathrm{C}\right)$. The unique cysteine-rich caps have lead to speculation that nyctalopin may not behave as other members of the SLRP family.

Biological roles for most SLRP proteins remain elusive, though several are reported to be involved in forming the structure of the extracellular matrix, mediating cell adhesion and regulating growth factor activity (Hocking et al., 1998; Winnemoller et al., 1991; Yamaguchi et al., 1990; Hildebrand et al., 1994). Nyx ${ }^{\text {nob }}$ mice lack nyctalopin and have a no b-wave ERG phenotype indicating disrupted signaling between photoreceptors (PR) and depolarizing bipolar cells (DBC) (Pardue et al., 1998). Glutamate puffs in the OPL of $N y x^{\text {nob }}$ mice are unable to elicit a DBC response (Gregg et al., 2007), which suggests nyctalopin is directly involved in the Grm6 cascade that regulates the TRPM1 channel. The $N y x^{\text {nob }}$ mouse has normal retinal morphology and ultrastructure suggesting nyctalopin is not affecting the structure of the extracellular matrix like many SLRP proteins (Pardue et al., 1998, 2001; Ball et al., 2003).

Nyctalopin is predicted to be extracellular, so interactions likely occur in the extracellular matrix. In the DBCs, there are two known proteins with extracellular domains that are involved in generating the light-evoked response, Grm6 and TRPM1. In the previous chapters, I showed that accumulation of nyctalopin in the DBC dendritic tips co-localizes with Grm6 and TRPM1 (Gregg et al., 2007; Pearring et al., 2010). Since all three proteins are closely associated in DBC dendrites and nyctalopin is required for proper signaling of TRPM1 by Grm6, I hypothesize that nyctalopin will interact with Grm6 or TRPM1.

Grm6 receptors form dimers to transmit external glutamate signals to an intracellular Gprotein signaling pathway. The Grm6 protein consists of three distinct regions: the extracellular $(\mathrm{EC})$, transmembrane (TM), and intracellular regions. The large $\mathrm{EC}$ region recognizes glutamate and can be further divided into two parts: the $\mathrm{N}$-terminal ligand binding region (LB) and the cysteine-rich (CR) domain. The large LB region of Grm6 undergoes a ligand-induced 
conformational change when glutamate binds (Muto et al., 2007). The neighboring CR domains in the Grm6 dimer form lateral interactions, which are believed to promote clustering of the receptor to the cell surface. If nyctalopin bound these extracellular regions of Grm6 it could regulate ligand-binding or cluster the receptor to the DBC dendritic tips.

To date no structural information is available for the TRPM1 channel. Electron microscopic reconstruction of three TRP members, TRPC3, TRPM1 and TRPV1 provides a general structure for TRP channels (Maruyama et al., 2007; Mio et al., 2005; Moiseenkova-Bell et al., 2008). TRPM 2 and TRPC3 channels have a bullet-shape with a dense putative transmembrane domain and the majority of the protein occupying the cytosol (see review by Moiseenkova-Bell \& Wensel, 2009). However, the amino acid sequence variety among TRP channels suggests they are likely to be a structurally diverse family.

Based on these TRP channel structures and transmembrane prediction programs, TRPM1 is not predicted to have a large extracellular domain. Interactions with nyctalopin would have to occur with the small extracellular domains or perhaps within the transmembrane region, which could only be possible for the murine version of nyctalopin. Therefore, it is likely that the extracellular domain of nyctalopin interacts with its binding partner. Interdependence experiments reported in Chapter III show that nyctalopin is required for expression of TRPM1 to the dendritic tips of DBCs. An example of TRP channel targeting to the membrane has been described in Drosophila. INAD, Inactivation No After Potential D, regulates the subcellular localization and stability of the TRP channel in the rhabdomere (Tsunoda et al., 1997; Chevesich et al., 1997), which is critical to phototransduction. Interactions between nyctalopin and TRPM1 could play a role in regulation, subunit assembly or trafficking of the TRPM1 channel in the DBC dendritic tips. 
In this chapter, I investigate the role of nyctalopin interactions with the Grm6 receptor and the TRPM1 channel to further elucidate its function.

\section{Results}

\section{Purification of YFP-nyctalopin from TgYFPNYX murine retina}

To examine interacting partners of nyctalopin it is essential to be able to identify and purify nyctalopin complexes from total retinal protein. Many attempts included several different strategies to isolate YFP-nyctalopin from transgenic mouse retinas that expressed the YFPnyctalopin fusion protein (TgYFPNYX). None of these approaches were successful. Retinas were subjected to a variety of methods such as deglycosylation to remove large glycans from the nyctalopin protein (for a complete list, refer to Table 7 in the methods section), membrane fractionation and a range of strong and weak detergents. Regardless of the approach I was unable to detect YFP-nyctalopin on a western blot. Nyctalopin is predicted to be glycosylated, therefore, it is possible its in vivo molecular weight was too large to enter the polyacrylamide gel, or be transferred to the PVDF membrane. In an attempt to decrease the molecular mass of nyctalopin I scanned the protein for protease cleavage sites. YFP-nyctalopin contains two predicted thrombin cleavage sites that upon exposure to thrombin should liberate the YFP-tag. However, these sites were not recognized by thrombin in my control experiments. Thrombin experiments are described in more detail in Appendix I.

Figure 24 shows a representative western blot from control $(C 57 B / / 6 J)$ and TgYFPNYX retina lysate following membrane fractionation. Each fraction, total lysate (L), cytoplasmic (C) and membrane (M) was separated by sodium dodecyl sulfate polyacrylamide gel electrophoresis (SDS-PAGE) then analyzed by immunoblot. Lysates from mock or transiently transfected recombinant Strep-YFP-tagged nyctalopin HEK293T cells were loaded as negative and positive controls, respectively (lanes 1-2, Figure 24A). Complete details of the generation, expression and 
purification of Strep-YFP-nyctalopin (SYFP-NYX) from HEK293T cells are discussed in detail in the next section. Immunoblotting with a GFP antibody identified a band in the lane with recombinant YFP-nyctalopin at the predicted size $90 \mathrm{kDa}$.

The next three lanes contain protein from the membrane fraction isolated from retinas of $C 57 \mathrm{~B} / / 6 \mathrm{~J}$ mice. As expected, blotting with a GFP antibody did not detect any proteins in the C57BI/6J fractions (lane 3-5, Figure 24A). Lanes 6-8 contain membrane fractions from TgYFPNYX retina. Surprisingly, we could not detect a band for YGP-nyctalopin in the TgYFPNYX total lysate or membrane fraction (Figure 24A). To ensure the blot contained protein, it was stripped and reprobed with an antibody to the ribbon synapse protein, RIBEYE. The RIBEYE antibody recognizes two protein bands (110 and $90 \mathrm{kDa}$, Figure 24B) in the mouse retina (tom Dieck \& Brandstätter, 2005). The RIBEYE bands followed the expected pattern for membrane proteins by being present in total lysate and enriched in the membrane fraction, but not present in the cytoplasmic fraction. These results show isolation and enrichment of membrane proteins from the TgYFPNYX murine retina; however, I was unable to detect YFP-nyctalopin. 


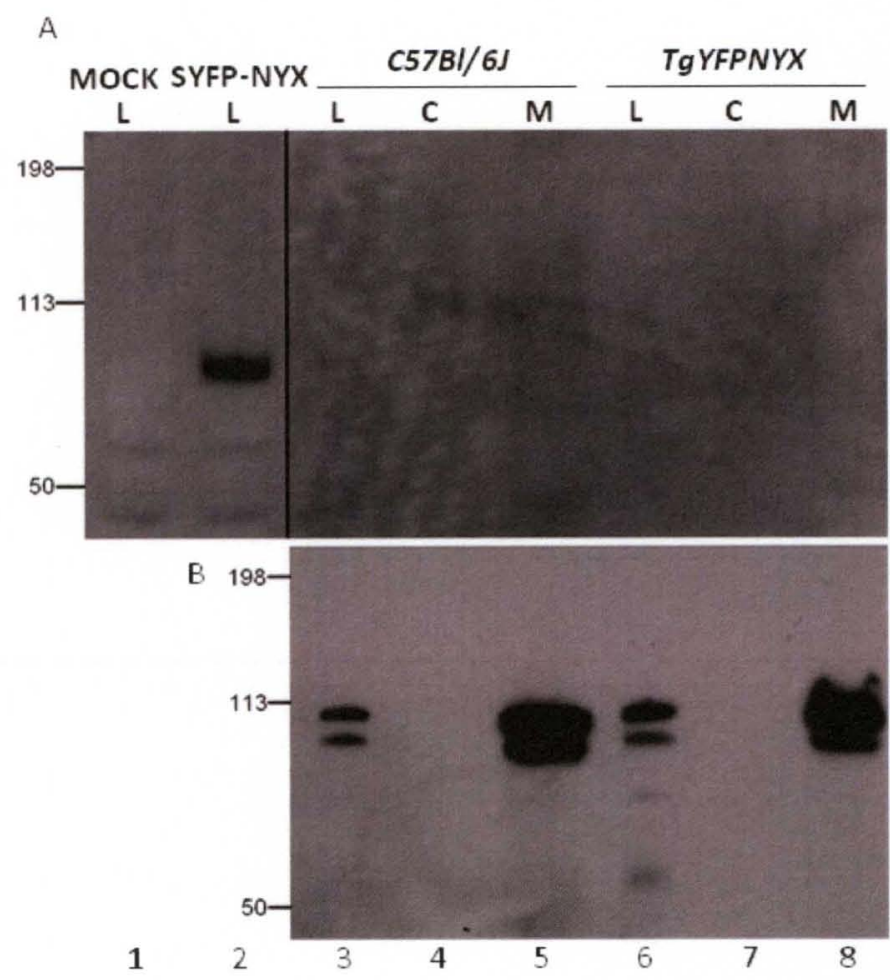

Figure 24: Western blot for YFPnyctalopin. (A) GFP antibody detects recombinant SYFP-NYX expression from HEK293T cells (lane 2), but not in $C 57 \mathrm{Bl} / 6 \mathrm{~J}$ (lanes 3-5) or TgYFPNYX (lanes 6$8)$ retina fractions. (B) To confirm the blot contained protein the same blot was re-probed with an antibody for the synaptic ribbon protein, RIBEYE. Lysate (L), cytosolic fraction (C), and membrane fraction (M)

Immunohistochemistry in TgYFPNYX retinal sections using a GFP antibody shows YFPnyctalopin expression is restricted to DBC dendritic tips in the OPL (refer to Figure 16, Gregg et al., 2007). Therefore, the amount of YFP-nyctalopin expressed may be a small fraction of total protein. I used an immunoprecipitation approach to enrich for YFP-nyctalopin from total TgYFPNYX retinal lysate. Protein-G agarose beads (Roche, Basel, Schweiz) were added to the pre-clear the lysate. This will identify YFP-nyctalopin if it was non-specifically bound to the agarose beads. After the pre-clearing the supernatant, $1 \mu \mathrm{g}$ of GFP antibody was incubated with the TgYFPNYX lysate. Then Protein-G agarose beads were added to precipitate the antibody and any bound proteins. The GFP antibody was incubated with the protein lysate before adding the Protein-G agarose beads to prevent the antigen site from being blocked. Finally, the beads with attached antibody were washed to remove non-interacting proteins. Bound proteins were 
released and denatured by heating to $70^{\circ} \mathrm{C}$ in sample loading buffer before separation by SDSPAGE.

Figure 25 shows a western blot using this immunoprecipitation approach. As a positive control for the GFP antibody, lysate from SYFP-NYX expressing HEK293T cells was loaded in the first lane of the gel and the GFP antibody detects the expected band for SYFP-NYX (lane 1, Figure 25A). In contrast, the GFP antibody did not detect YFP-nyctalopin in any fraction from the TgYFPNYX immunoprecipitation (lane 2-5, Figure 25A). To ensure the protein lysate was not degraded during immunoprecipitation, the membrane was stripped and re-probed with RIBEYE antibody (Figure 25B). The presence of RIBEYE bands confirm protein was loaded onto the polyacrylamide gel. Additionally, RIBEYE did not bind the elution fraction (E) off the GFP antibody.

Expression of YFP-nyctalopin mRNA is highest during synaptogenesis, specifically at post-natal day 13 (P13) (Bojang \& Gregg, 2007). Immunoprecipitation experiments also were performed on retinas collected from TgYFPNYX mice at P13 to optimize for the peak expression of nyctalopin. Even at the peak of nyctalopin mRNA expression this approach produced negative results (data not shown). 




Figure 25: Immunoprecipitation results from TgYFPNYX retina lysate. Total lysates (L), pre-clearing off the Protein$G$ beads (pre), elution from the GFP antibody (E) and flow-through fraction that did not bind antibody (FT) were loaded to track YFP-nyctalopin in the immunoprecipitation experiment. (A) In the first lane, YFP-nyctalopin from HEK293T cells was loaded as a positive control. The GFP antibody detected a band at 90KDa. The GFP antibody did not detect any bands for YFPnyctalopin. (B) Immunoblot with an anti-RIBEYE antibody. Bands for RIBEYE at 100 and $90 \mathrm{KDa}$ were present in the $\mathrm{L}$, Pre and FT fractions. RIBEYE does not interact with the GFP antibody, so is not present in the $E$ fraction. 
membranes (lane 1, Figure 26). No SYFP-NYX band was identified in the flow-through fraction, probably because SYFP-NYX lysate was diluted into the retinal membrane fraction. The GFP antibody did not pull down all the spiked SYFP-NYX and did not detect any bands in any of the fractions from the immunoprecipitation experiment on TgYFPNYX membranes (lane 5-7, Figure 26). Recombinant SYFP-NYX was not completely purified from the retina, which could be due to SYFP-NYX binding retinal proteins that prevent interactions with the GFP antibody. These data clearly show that my procedures could pull down at least a fraction of YFP-nyctalopin. The reason I am unable to enrich YFP-nyctalopin from the TgYFPNYX retina lysates remains unclear.

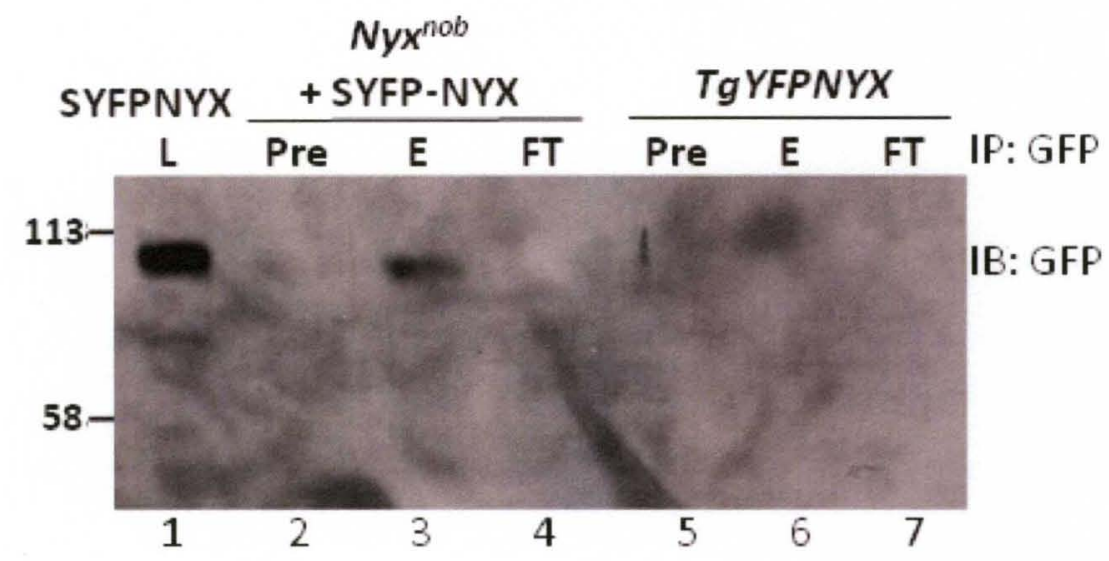

Figure 26: Western blot of protein fractions from GFP immunoprecipitation. Pre-clearing off Protein-G beads (Pre), elution off GFP antibody (E), flow-through fraction not bound to antibody (FT). Lane 1, total recombinant SYFP-NYX lysate (L). Lane $2-4$, Recombinant SYFP-NYX spiked into $N y x^{\text {nob }}$ retina lysate. GFP antibody detected SYFP-NYX in elution fraction (E). Lane 5-7, GFP antibody did not detect any bands for YFP-nyctalopin in the immunoprecipitation from TgYFPNYX membrane fractions.

\section{Strep-YFP-Nyctalopin Expression}

To overcome the problems of purifying nyctalopin from retinal tissue, I constructed a recombinant nyctalopin to be expressed in HEK293T cells. A Strep-tag II (WSHPQFEK) was added to YFP-nyctalopin to provide an additional purification option. The complete construct contains, from $\mathrm{N}$ - to C-terminus, nyctalopin's signal sequence, Strep-tag II, YFP tag and full-length nyctalopin with its LRR domain, conserved domain and transmembrane domain (Figure 26A). 
This construct will be referred to as SYFP-NYX. SYFP-NYX was transfected into HEK293T cells and its expression pattern examined by immunocytochemistry. Staining of the transfected cells with a GFP antibody shows robust expression of SYFP-NYX (Figure 26). Double labeling experiments were performed to examine sub-cellular localization of SYFP-NYX. The nucleus of the HEK293T cells was stained with a mono-nucleic acid stain, ToPro3-iodide, their plasma membranes with an antibody to $\mathrm{Na} / \mathrm{K}$-ATPase and their endoplasmic reticuli (ER) with an antibody to calnexin. Figure 27B and $27 \mathrm{C}$ show the co-localization of these subcellular markers with GFP. The merged images at the bottom show that SYFP-NYX does not co-localize with the nuclear marker, ToPro3 or with the plasma membrane marker, NA/K-ATPase. However, SYFP-NYX and calnexin display strong co-localization as shown in the merged image (Figure 27D). These data suggest that the majority of SYFP-NYX is restricted to the ER in HEK293T cells. 




Leucine-Rich Repeats

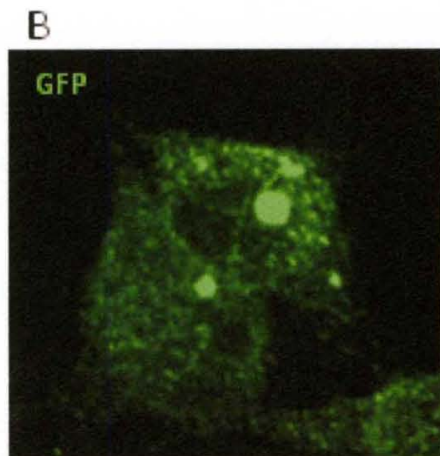

C

D


Figure 27. SYFP-NYX expression in HEK293T cells. (A) Schematic of the SYFP-NYX construct transiently expressed in HEK293T cells. Downstream of the signal sequence (purple) the Strep (blue) and YFP (yellow) tags were added to the $\mathrm{N}$-terminus of murine nyctalopin. The LRR regions (red) are flanked by cysteine rich repeats (dark blue). The conserved region (pink) and transmembrane domain (green) are at the C-terminus. (B-D) Immunocytochemistry for SYFP-NYX was performed using a GFP antibody (green) and double labeled with a nuclear dye, ToPro3 (red, B) and antibodies to the plasma membrane, Na-K ATPase (red, C) or the ER marker calnexin (red, D). SYFP-NYX co-localizes with calnexin staining in the ER, shown by yellow in the merged image in (D) Scale bar is $10 \mu \mathrm{m}$ 


\section{Purification of Strep-YFP-Nyctalopin}

Isolation of recombinant SYFP-NYX from total HEK293T cell lysate is required to perform interaction studies. SYFP-NYX can be purified by the Step-tag II or by the YFP tag, both methods were tested.

Purification of the SYFP-NYX protein from HEK293T cell lysate can be achieved using strep-tactin conjugated magnetic beads. The strep-tactin magnetic beads have a very high affinity for the Strep-tag II tag $\left(K_{d}=1 \mu \mathrm{M}\right)$, which is nearly 100 times higher than to streptavidin (Strep-tagged Protein Purification Handbook, Qiagen, Valencia, CA). Proteins can be eluted by addition of a buffer containing $10 \mathrm{mM}$ biotin. An western blot from a strep-tactin pull down from a lysate prepared from SYFP-NYX transfected HEK293T cells is shown in Figure 28A. Immunoblotting was done using a GFP antibody, which detects a band for SYFP-NYX at $90 \mathrm{kDa}$ as expected (lane 1). The elution fraction containing proteins bound to the strep-tactin beads and is enriched with SYFP-NYX (lane 2, Figure 28A). The flow-through represents proteins that did not bind to the strep-tactin beads and contains a small amount of SYFP-NYX (lane 3, Figure 28A). This latter fraction contained SYFP-NYX because the strep-tactin beads were saturated. In addition to the band at $90 \mathrm{kDa}$, the GFP antibody detected a band at $60 \mathrm{kDa}$ in the elution fraction. This band is also present in the elution fraction from mock transfected HEK293T cell lysates and represents non-specific labeling by the GFP antibody.

An alternative method to isolate SYFP-NYX protein from HEK293T cell lysate is to use a GFP antibody. Complexes bound to the GFP antibody are precipitated using Protein-G agarose beads. Figure 28B shows the immunoprecipitation fractions from mock transfected HEK293T cells (mock, lanes 1-4) and HEK293T cells transfected with SYFP-NYX (lanes 5-8). Immunoblotting shows the GFP antibody does not detect any bands in the mock transfected fractions (Figure 28B). However, a $90 \mathrm{kDa}$ band, representing SYFP-NYX, is present in the total lysate from the 
transfected cells (L, lane 5, Figure 28B). Immunoprecipitation of SYFP-NYX with the monoclonal GFP antibody shows enrichment for SYFP-NYX in the eluted fraction (E, lane 6, Figure 28B). The pre-clearing fraction (Pre) contains proteins that are bound non-specifically to the Protein-G agarose beads (lane 7, Figure 28B) and this fraction contains a small amount of SYFP-NYX. This shows that SYFP-NYX interacts non-specifically with the Protein-G beads, though the GFP antibody does enrich for SYFP-NYX (E). The flow through (FT) fraction represents proteins that did not bind the GFP antibody or Protein-G beads. A small amount of SYFP-NYX is present in this fraction suggesting the GFP antibody was saturated with SYFP-NYX.

The above results demonstrate that recombinant SYFP-NYX protein expression in HEK293T cells can be purified using strep-tactin magnetic beads or by immunoprecipitation with a GFP antibody.
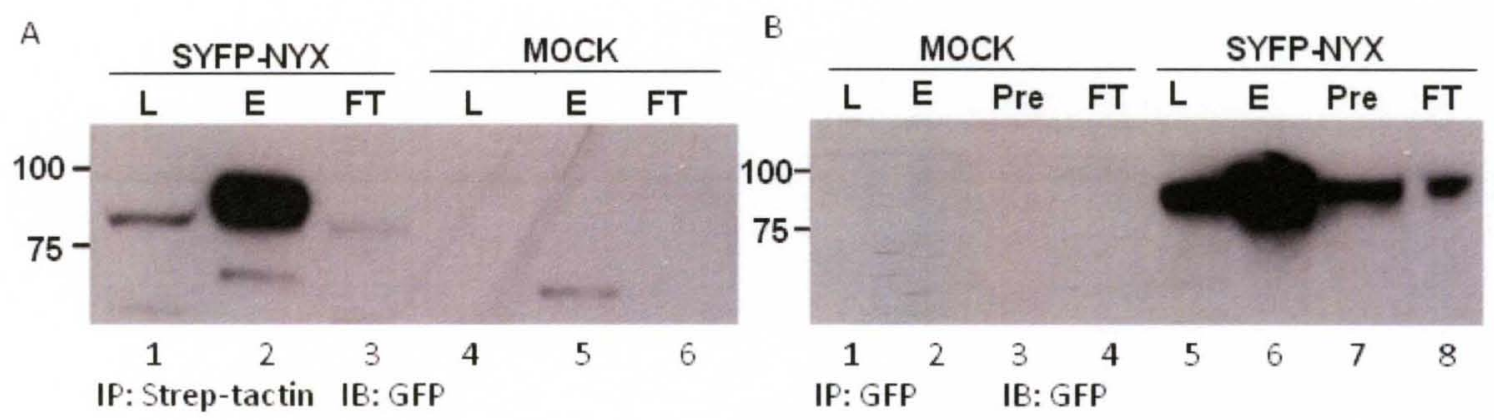

Figure 28. Nyctalopin purification from transiently transfected HEK293T cells. (A) Strep-tactin pull down. Immunoblott using a GFP antibody shows specific expression of SYFP-NYX in the lysates (L) at 90KD, lane 1. SYFP-NYX is enriched when eluted off strep-tactin beads (lane 2, E) though a small fraction remains in the flow-through (lane 3, FT). Mock transfected control is present in lane 4-6 and shows a nonspecific band at 60KDa (B) Immunoprecipitation of SYFP-NYX using a monoclonal GFP antibody (MBL). Negative control is loaded in lanes 1-4. SYFP-NYX is highly expressed in the lysate (lane 5, L), but enriched by the immunoprecipitation (lane 6, E). A small fraction of SYFP-NYX nonspecifically interacts with the pre-clearing fraction (lane 7, pre) as well as remains in the flow-through (lane 8, FT). 


\section{Interactions between Nyctalopin and Grm6}

Nyctalopin is required for the signal transduction between Grm6 and the TRPM1 channel in DBCs. To examine whether nyctalopin interacts with Grm6 a recombinant SYFP-NYX column was made. To generate the SYFP-NYX column, total lysate from HEK293T cells transfected with SYFP-NYX was harvested and immunoprecipitated with a monoclonal anti-GFP antibody. Several washes were performed to remove unbound proteins from the Protein-GSYFP-NYX column before adding rabbit retina lysate to determine if Grm6 could bind SYFP-NYX. Rabbit retina was used because the antibody to detect Grm6 was specific for rabbit Grm6. Western blot shows a positive Grm6 band from total rabbit retina lysate (lane1, Figure 29A). There is no band detected by the GFP antibody in the rabbit retina lysate (lane 1, Figure 29B).

Protein fractions from generating the SYFP-NYX column and eluting off the column were collected and analyzed by western blot. A GFP (Figure 29B) and rabbit-specific Grm6 (Figure 28A) antibody were used to follow the purification of SYFP-NYX and whether it interacted with Grm6. Figure 28A shows the Grm6 antibody only detected a band for Grm6 in the flow through fraction from the rabbit retina lysates (Rab FT, lane 4). The GFP antibody detected SYFP-NYX in the elution (E) fraction from the column (lane 5, Figure 29B). Detection of SYFP-NYX in the elution (E) fraction indicates enrichment for SYFP-NYX on the column. Additionally, a small fraction of SYFP-NYX was bound to the pre-clearing fraction, as described in the previous section. These results show that I was unable to obtain any evidence for interactions between Grm6 and SYFP-NYX in this in vitro system. This in vitro interaction study was attempted using three different buffers with similar results. Buffers are described in the methods section (data not shown). 


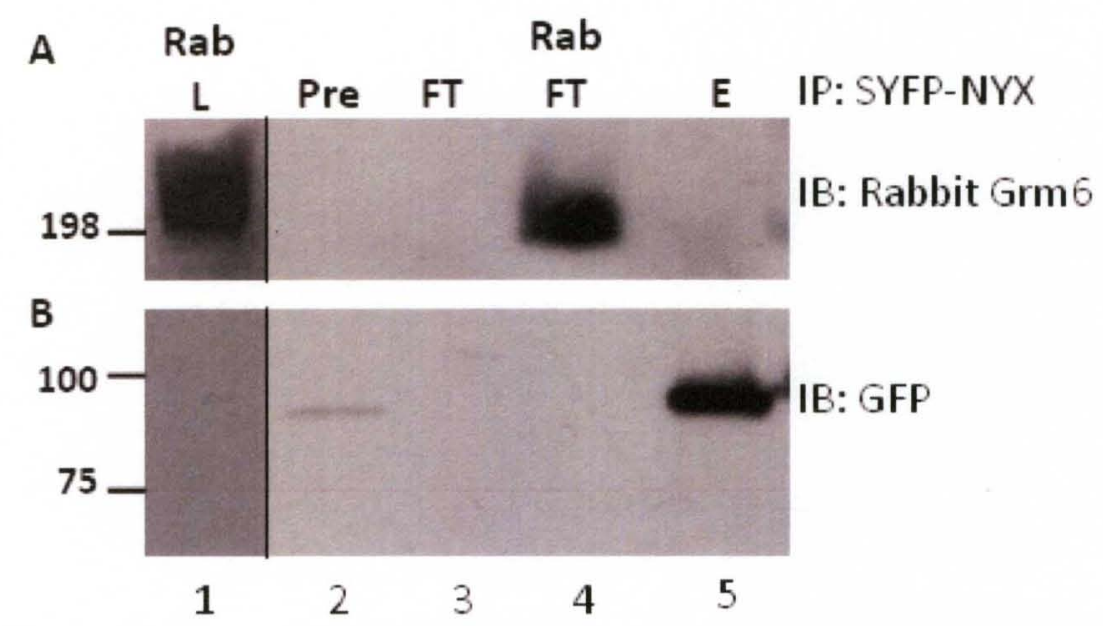

Figure 29: Western blot of in vitro binding study of SYFP-NYX and Grm6. SYFP-NYX column was generated by immunoprecipitation with a GFP antibody. Upper panel is immunoblot with a GFP antibody. SYFP-NYX is detected in pre-clearing fraction (pre) and enriched in the fraction eluted off GFP antibody (E). Lower panel is an immunoblot with a rabbit Grm6 antibody. Grm6 is present in rabbit retina lysate (Rab $\mathrm{L}$ ) as well as the flow-through from the rabbit retinal lysate (Rab FT), containing unbound proteins. Rabbit Grm6 does not interact with the SYFP-NYX bound protein.

\section{Interactions between Nyctalopin and TRPM1}

The TRPM1 channel in $\mathrm{Nyx}^{\text {nob }}$ mice is predicted to be absent or closed (Gregg et al., 2007) and nyctalopin is required for TRPM1 expression in the DBC dendritic tips (results from Chapter III). Therefore, I examined whether nyctalopin interacted with TRPM1 by doing coimmunoprecipitation experiments.

To perform interaction studies between TRPM1 and nyctalopin I used a heterologous expression system. A flag and GFP tag were added to the N-terminus of TRPM1-SS. The complete construct contains, from N- to C-terminus, the flag and GFP tag linked to full-length TRPM1 with its TRPM homology region, six transmembrane domains (including the predicted pore), TRP domain, coiled-coil domain and the C-terminus unique region (Figure 30A). This construct will be referred to as FG-TRPM1. HEK293T, CHO, and NG108-15 cell lines were tested for expression of FG-TRPM1 by immunocytochemistry, but all produced similar results so only the data from HEK293T cells will be discussed in detail. 
FG-TRPM1 was transiently transfected into HEK293T cells and expression was determined by immunocytochemistry using a GFP antibody (Figure 30). FG-TRPM1 was excluded from the nucleus, which was labeled with ToPro3-iodide (Figure 30B). FG-TRPM1 also did not colocalize with the plasma membrane marker, Na-K ATPase (Figure $30 \mathrm{C}$ ). This result suggests that the majority of FG-TRPM1 is most likely located in the ER, although, if only a few channels are expressed at the cell surface it may be difficult to detect. Double labeling for the ER marker calnexin confirmed that FG-TRPM1 is predominantly expressed in the ER (yellow in merged image, Figure 30D). These results are similar to the expression of SYFP-NYX in HEK293T cells (refer to Figure 27). Localization of both FG-TRPM1 and SYFP-NYX in the ER of HEK293T cells suggests that DBC proteins are not being properly trafficked to the plasma membrane in this heterologous system. Over expression of the protein by the CMV promoter may cause a bottleneck effect in the ER, prevent proper folding the proteins or HEK293T cells might not contain the proper trafficking machinery to deliver TRPM1 to the plasma membrane. While the intracellular localization of the two proteins was not as expected it is still possible they interact. 
A

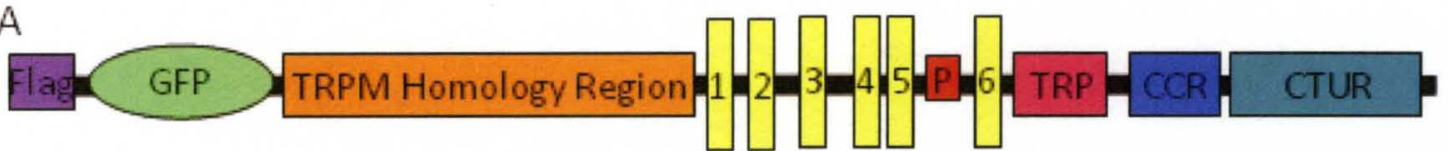

B

C

D
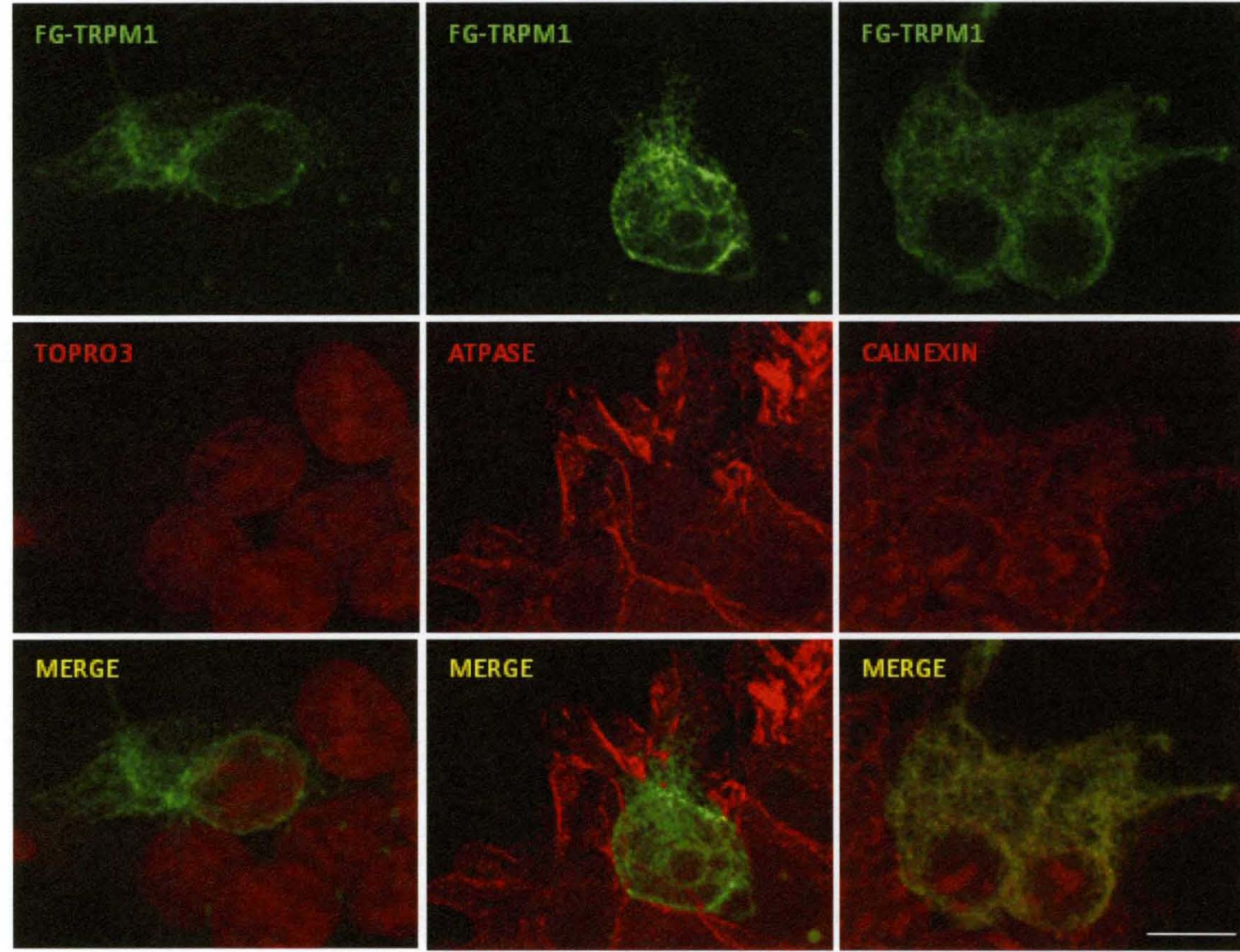

\section{MERGE}

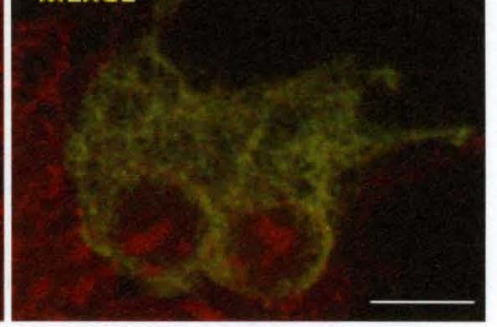

Figure 30. Expression of FG-TRPM1 in HEK293T cells. (A) Representation of the FG-TRPM1 construct. flag (purple) and GFP (green) were added to N-terminus of TRPM1 followed by the TRPM homology region (orange), 6 transmembrane domains (yellow) that contain the putative pore (red), TRP domain (pink), coiled-coil domain (blue) and C-terminal unique region (aqua). (B-D) Immunocytochemistry for FG-TRPM1 was performed using a GFP antibody (green) and co-labeled with antibodies to the nucleus, ToPro3 (red, B); the plasma membrane, Na/K-ATPase (red, C), or the ER, calnexin (red, D). FG-TRPM1 only co-localizes with calnexin staining in the ER, shown by yellow in merged image (D) Scale bar is $10 \mu \mathrm{m}$ 
To determine if FG-TRPM1 and SYFP-NYX do interact, I transiently transfected HEK293T cells, prepared lysates and performed pull-downs with step-tactin magnetic beads as described previously. Four separate transfections were performed to include all the necessary controls. HEK293T cells were mock transfected (lanes 1-2), transfected with either SYFP-NYX (lanes 3-5) or FG-TRPM1 (lanes 6-8) or co-transfected with both SYFP-NYX and FG-TRPM1 (lanes 9-11, Figure 31).

For each transfection I prepared fractions containing the total lysate $(L)$, strep-tactin bound proteins (E) and unbound proteins (FT) and analyzed then by western blot. Figure 31 shows the results of the western blot using a flag antibody for FG-TRPM1 (Figure 31A) and a GFP antibody for SYFP-NYX (Figure 31B). To confirm the bound fractions were purified from the total lysate the polyacrylamide gel was stained with coomassie blue (Figure 31C). Total HEK293T lysate contains hundreds of bands, which are no longer present in the elution fractions.

In the mock lysate the flag antibody did not detect any bands. A non-specific band at 60 kDa can be seen with the GFP antibody in the bound fraction (lane 2, Figure 31B). This nonspecific band is in every fraction eluted from the strep-tactin beads and its identity is unknown. The SYFP-NYX lysate contains a $90 \mathrm{kDa}$ band representing SYFP-NYX (lane 3, Figure 31B). This SYFP-NYX is greatly enriched in the elution fraction (E) as can be seen by the increase in band intensity (lane 4, Figure 31B) and corresponding decrease in total protein as shown by coomassie staining (Figure 31C). No bands are detected in the SYFP-NYX lysate with the flag antibody (lane 3-5, Figure 31A). The FG-TRPM1 lysate (L) contains a $200 \mathrm{kDa}$ band for FG-TRPM1 that is also present in the unbound fraction (lanes 6-8, Figure 31A). When FG-TRPM1 and SYFPNYX are co-transfected a fraction of FG-TRPM1 is detected in the bound fraction by the flag antibody (lane 10, Figure 31A). This bound fraction is enriched with SYFP-NYX that is pulling 
down the FG-TRPM1 (lane 10, Figure 31B). These results suggest that FG-TRPM1 is interacting with SYFP-NYX when purified from total lysate by the strep-tactin system.

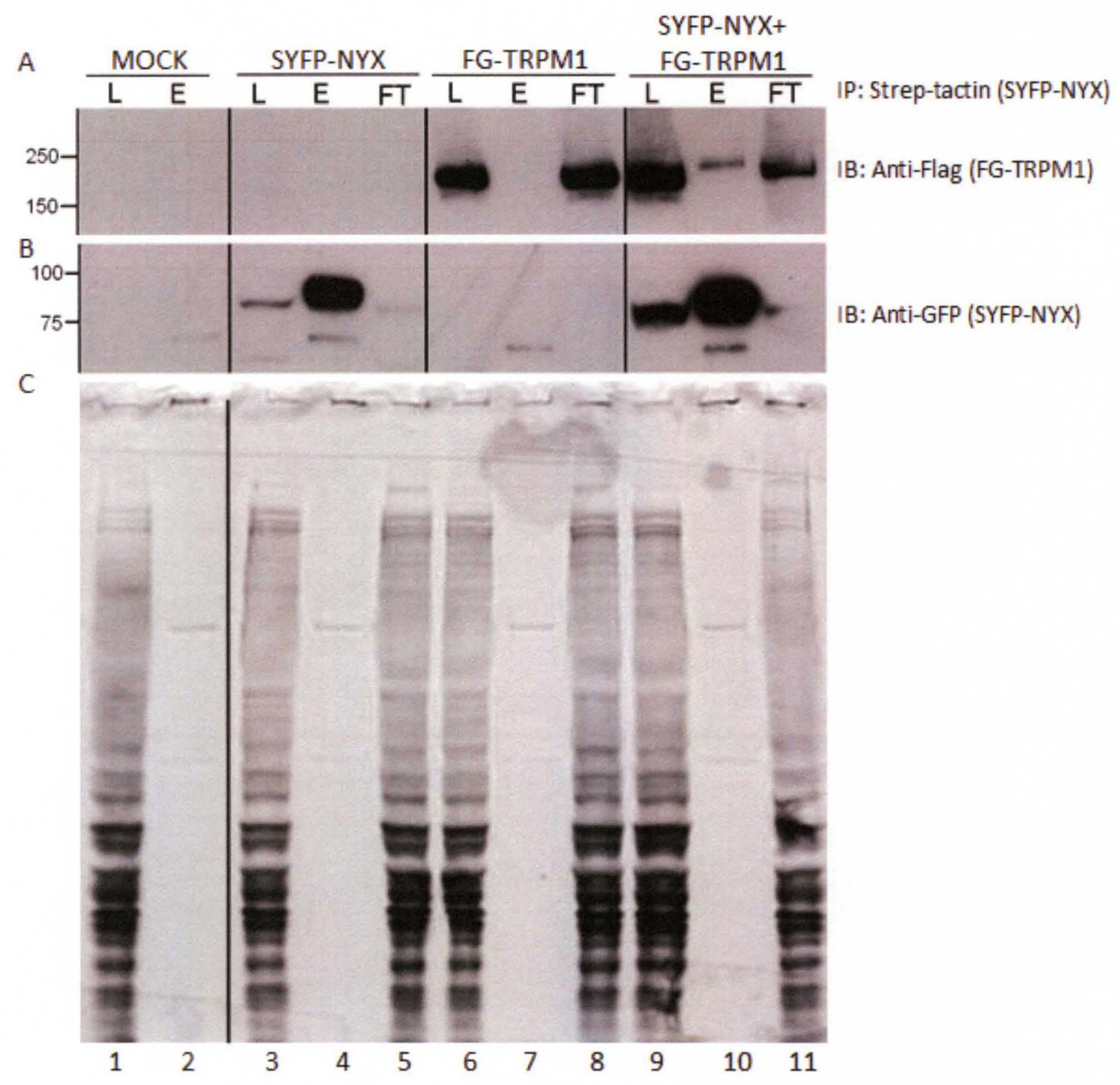

Figure 31: Nyctalopin in vitro interactions with TRPM1. (A) Western blot using an anti-flag antibody shows FG-TRPM1 is not bound to the strep-tactin magnetic beads (E) when expressed alone. However, a small fraction of FG-TRPM1 does bind when co-transfected with SYFP-NYX. (B) Western blot with a GFP antibody to identify SYFP-NYX elution off the strep-tactin magnetic beads (E). (C) Coomassie stained gel shows protein isolation from strep-tactin magnetic bead elution. Total Lysate (L), elution (E) and flow through (FT) from strep-tactin magnetic beads. 


\section{Functional Interactions of Nyctalopin and TRPM1}

In the previous section I showed when nyctalopin and TRPM1 are expressed in HEK293T cells they interact. To examine if this interaction contributes to the function of the TRPM1 channel I performed patch clamp recordings of HEK293T cells. A stable cell line expressing HATRPM1 was produced to reduce the variability of TRPM1 expression levels observed in transient transfection studies. The HA tag is 9aa (YPYDVPDYA) and should not interfere with normal channel function.

The TRPM1 channel in DBCs is opened by capsaicin and closed by capsazepine (Shen et al., 2009). I used this agonist and antagonist to directly modulate the TRPM1 channel in the HATRPM1 stable cell lines. In rod BCs, recording of the TRPM1 channel were done by holding the cells at positive voltages (Snellman et al., 2008; Shen et al., 2009). When the channel opens and outward current is generated. Therefore, whole-cell recordings from HEK293T cells were performed at a holding potential of $+50 \mathrm{mV}$.

Initial patch clamp experiments were performed in mock transfected HEK293T cells to confirm that capsaicin and capsazepine did not produce a current from an endogenous channel (Figure 32A). Representative current recordings from a HEK293T cell exposed to a $3 \mathrm{sec}$ puff of $20 \mu \mathrm{M}$ capsaicin (blue) and 20 $\mu \mathrm{M}$ capsazepine (red) are shown in Figure 32A. Neither drug elicited a current confirming HEK293T cells do not contain channels that respond to capsaicin or capsazepine $(n=8)$.

Whole-cell recordings from HA-TRPM1 cells show that the capsaicin agonist did not generate a current (blue trace, Figure 32B). However, application of the TRPM1 antagonist, capsazepine, elicited a small inward current in HA-TRPM1 expressing cells (red trace Figure 32B). For each condition 8 HEK239T control cells, 15 HA-TRPM1 stable cells and 15 HA-TRPM1 expressing YFP-nyctalopin cells were tested. The mean and standard error of the peak response, 
time to peak and rise time are plotted in Figure 32D. The TRPM1 channel in HEK293T cells does not respond to agonist, but responds with inward current to its antagonist. Inward currents when holding at a positive potential suggests the antagonist is closing the channel. These results imply that the TRPM1 channel is constitutively active in HEK293T cells.

To determine if nyctalopin affected TRPM1 currents SYFP-NYX was transfected into the HATRPM1 stable cells. SYFP-NYX expressing cells were identified by YFP fluorescence before wholecell recordings. Recordings from 13 cells were obtained and Figure $32 \mathrm{C}$ shows a representative capsazepine response from SYFP-NYX transfected HA-TRPM1 stable cell. The capsazepine current in the SYFP-NYX transfected HA-TRPM1 cells was indistinguishable from HA-TRPM1 alone. No significant difference was detected between peak responses, time to peak or rise time between HA-TRPM1 and HA-TRPM1 with SYFP-NYX current (Figure 32D, E, F, respectively). This suggests that either nyctalopin does not modulate TRPM1 channel currents or is not involved in inserting TRPM1 into the plasma membrane in this heterologous system. T values for peak response, time to peak and rise time are shown in Table 5.

Table 5: Capsazepine Response Statistics

\begin{tabular}{lcc}
\hline Protocol & T Value & T Probability \\
\hline Peak Response & 0.53 & 0.60 \\
Time to Peak & 0.62 & 0.54 \\
Rise Time & 0.01 & 0.99 \\
\hline
\end{tabular}


А HEK293T
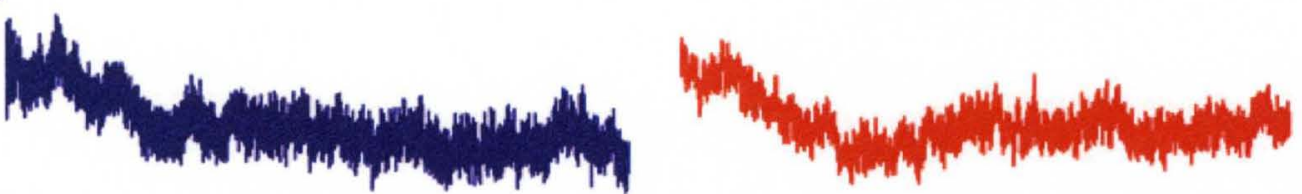

B HA-TRPM1

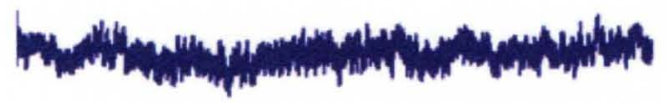

C HATRPM1+SYFP-NYX
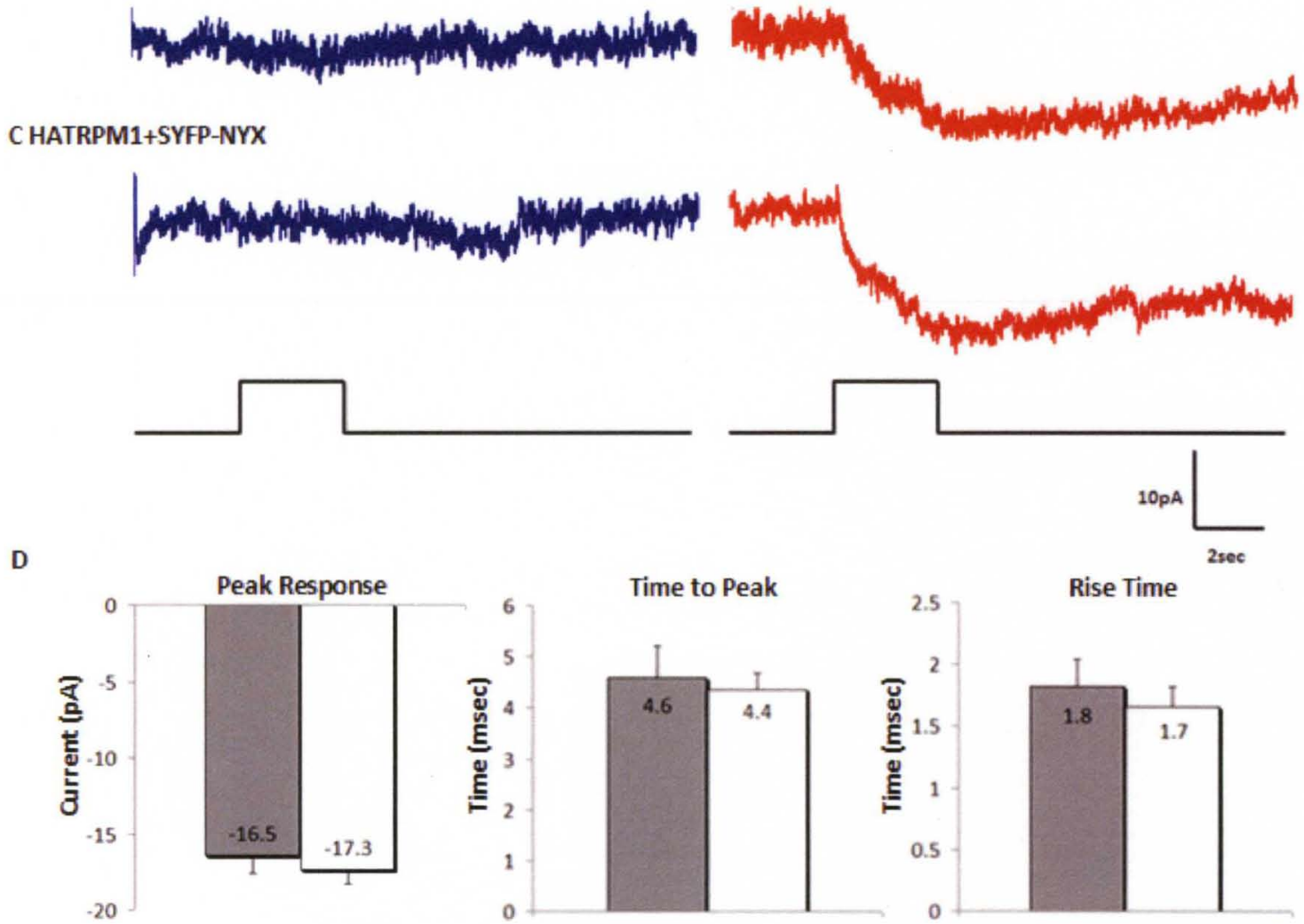

Figure 32: Whole cell patch clamp recordings from HEK293T cells. Representative current response of capsaicin (blue) and capsazepine (red) from a HEK293T cells $(n=8)(A)$, HA-TRPM1 stable cells (B) and HATRPM1 stable cells transiently transfected with SYFP-NYX (C). TRPM1 agonist, capsaicin, or antagonist, capsazepine, were applied for a 3 second puff and currents recorded for 60 seconds. Traces represent an average of 7 trials. HA-TRPM1 and HA-TRPM1+SYFPNYX average current responses to capsazepine were analyzed (D). Average peak response, average time to peak or average time to rise from baseline to peak for capsazepine responses were not significantly different between HA-TRPM1 $(n=15)$ and HATRPM1+SYFP-NYX $(n=13)$ cells. 


\section{Discussion}

In DBCs, the Grm6 receptor uses an intracellular G-protein cascade to regulate the TRPM1 channel. Nyctalopin is critical to maintain transduction of this signal, though it is an extracellular protein. Structure-forming proteins bring receptors and channels into close vicinity to help mediate signaling. The LRR domain of nyctalopin is primed for protein interactions, so it could be involved in forming a macromolecular complex in the DBC dendritic tips. I tested nyctalopin interactions to membrane bound proteins, Grm6 and TRPM1.

Purification of YFP-nyctalopin from TgYFPNYX retinas proved to be difficult. I attempted to enrich for YFP-nyctalopin by isolating membrane fractions as well as performing immunoprecipitation experiments. However, though the GFP antibody identified recombinant SYFP-NYX, it was unable to detect YFP-nyctalopin from retina. Either further enrichment is needed or YFP-nyctalopin is being bound by the extracellular matrix. Over expression of recombinant SYFP-NYX in HEK293T cells, which do not contain extracellular matrix, may not contain all the post-translational modifications added to nyctalopin in the DBCs. Nyctalopin has been shown to be heavily glycosylated (O'Conner et al., 2005). Computer-based predictions show human nyctalopin contains six putative $\mathrm{N}$-glycosylation sites and four O-glycosylation sites (Pesch et al., 2003; Zeitz et al., 2003). These added modifications could lead to increased molecular weight. Though I attempted to de-glycosylate YFP-nyctalopin using a variety of deglycosylation enzymes, the lack of an internal control prevented me from determining if the deglycosylation was working on retina lysate.

Patch clamp recordings from $N y x^{\text {nob }}$ DBCs show that Grm6 signaling to the TRPM1 channel is disrupted. Nyctalopin could be directly interacting with Grm6 to participate in signal transduction. Interactions between rabbit Grm6 and a SYFP-NYX column could not be detected. However, if the rabbit Grm6 is already bound to endogenous rabbit nyctalopin it may be unable 
to bind the recombinant SYFP-NYX column. In addition, the species differences between the rabbit Grm6 and murine nyctalopin may prevent interaction between the two proteins. Despite these issues, the result agrees with yeast-two hybrid analysis performed between full-length nyctalopin and Grm6 (Bojang et al., 2009). Therefore, it appears nyctalopin does not participate in the $\mathrm{DBC}$ response by binding the Grm6 receptor.

On the other hand, nyctalopin could regulate channel opening by directly binding to the TRPM1 channel. Then in the absence of nyctalopin, TRPM1 would be constitutively closed as suggested by the patch clamp recordings (Gregg et al., 2007). In vitro binding studies performed resulted in interactions between nyctalopin and TRPM1. These results suggest that nyctalopin interactions with TRPM1 and could be regulating channel function or localization. Recombinant forms of TRPM1 and nyctalopin did not localize to the plasma membrane in HEK293T cells. Therefore, interactions between the two proteins are likely to occur in the ER.

Nyctalopin interactions with the TRPM1 channel were functionally investigated in a heterologous system. The TRPM1 antagonist, capsazepine, elicited small inward currents, while no current was observed during application of the TRPM1 agonist, capsaicin. These specific TRPM1 currents suggest that TRPM1 is constitutively active in HEK293T cells, which is consistent with a previous report that showed transient transfection of TRPM1 into HEK293T cells produced higher basal level of calcium (Xu et al., 2001). Surprisingly, the addition of nyctalopin in the TRPM1 stable cells did not alter TRPM1 capsazepine currents. Therefore, nyctalopin does not modulate the TRPM1 channel in this heterologous system.

Interdependence experiments from Chapter III suggest nyctalopin is required for proper expression of TRPM1. From these results I hypothesized nyctalopin might be involved in trafficking TRPM1 to the plasma membrane. Therefore, I expected nyctalopin to increase TRPM1 membrane expression resulting in larger capsazepine currents. Nyctalopin and TRPM1 are not 
properly localized in HEK293T cells. Both have high expression in the ER suggesting that the machinery to traffic DBC proteins is absent from this heterologous system. On the other hand, if nyctalopin expression did cause trafficking of the TRPM1 channel to the membrane and the channel is constitutively open in this system the cells could be dying from calcium overload. Though this is a possibility, nyctalopin positive cells were identified and shown to have normal TRPM1 currents, so at least a population of cells does not cause cell death.

It is important to note that activation and regulation of TRP channels is likely a complex process. For example, the TRP system in Drosophila requires a huge complex of proteins ranging from kinases to G-proteins to maintain normal TRP channel responses (Popescu et al., 2006; reviewed by Montell, 2005). These macromolecular complexes are difficult to study in heterologous systems in which critical components are missing or exist in an abnormal stoichiometry. Therefore, determining functional interactions in the in vivo system would provide the proper architecture to determine if nyctalopin affects TRPM1 currents. Appendix II describes initial in vivo electroporation experiments to transiently express YFP-nyctalopin in the retina. Using this approach, TRPM1 and nyctalopin could be manipulated in their native environment. In addition, mutant mice are available and would provide a means to study the proteins in a null background.

Collectively, my results suggest that nyctalopin and TRPM1 interact and that this interaction is required for proper expression of TRPM1 to the dendritic tips of DBCs. 


\section{CHAPTER V: METHODS}

Animals: All experiments were performed using protocols approved by the Animal Care and Use Committee at each institution and the guidelines of the National Institutes of Health. Mice were housed in a 12:12 light:dark cycle. For all experimental manipulations, mice were killed by anesthetic overdose or carbon dioxide exposure.

The $N y x^{\text {nob }}$ mutation, originally discovered on the BALBC/ByJ strain (Pardue et al. 1998), has been moved to a $C 57 B / / 6 J$ background by more than seven generations of backcrossing. C57BH/6J mice were provided by The Jackson Laboratory, Bar Harbor, ME. The Grm6 ${ }^{\text {nob4 }}$ mice were obtained from Neuromice (www.neuromice.org). TRPM1 ${ }^{-}$mice were generated by Lexicon Genetics (TRPM $1^{\text {tm1lex }}$ ) and obtained from the European Mouse Mutant Archive. Molecular details of the targeted allele are available at http://www.emmanet.org/.

The $T g(\text { Gabrr1:EYFP-Nyx })^{R g g 1}$ transgene was constructed using PCR based strategies as previously described in Gregg et al., (2007). A promoter fragment, consisting of $8 \mathrm{~Kb}$ of the murine GABACp1 gene (Gabrr1, Accession NM_008075), was used to drive expression of the fusion gene in BCs. The open reading frame (ORF) of EYFP was inserted in-frame after the predicted nyctalopin signal sequence (amino acid 19, Gregg et al. 2003) followed by the ORF of a cDNA representing the murine Nyx gene (Accession NM_173415). To improve expression, a Kozak sequence (Kozak 1991) was added to the start codon and a rabbit $\beta$-globin fragment containing exon 2-intron 2-exon 3 and a polyadenylation signal (Brinster et al., 1985) were placed downstream of the EYFP-Nyx fusion construct. The final construct was verified by 
sequencing before transgenic mice were generated by standard pronuclear injection procedures. Tg(Gabrr1:EYFP-Nyx $)^{R g g 1}$ will be referred to as TgYFPNYX mice.

Murine Genotyping: Genotypes were identified by PCR of DNA isolated from tail biopsies. All primers are listed from the $5^{\prime}-$ to $3^{\prime}$-end. PCR to identify the YFP-nyctalopin transgene was performed with the following primers, XFP1: CACATGAAGCAGCACGACTTCT and XFP2: GGGTGTTCTGCTGGTAGTGGTC Nyx ${ }^{\text {nob }}$ PCR primers were as follows, Nob3: CGGCCTTCGACAATCTCTTCC and m-Nyx31: TTACTACAGGCAGTTACTCA- TGGCAGTCAA. TRPM1 ${ }^{\prime-}$ PCR primers were as follows, neo-3A: GCAGCGCATCGCCTTCTATC, Trp-081: GCATAGTCCATGGACCTAGC, Trp-082: TGCAGGTTTGATTCACATCAT. Grm6 ${ }^{\text {nob4 }}$ PCR primers were as follows, Grm6Rev: AGAGGCCAGTGTAGACACGTAATT and Grm6Bio: 5'-bio/AGT GAC TCC ACA CGC TAT GAC TT. The PCR products were then analyzed using the sequencing primer Grm6Seq: GGAGGCACCACTCGG on a Biotage, Uppsala, Sweden pyro-sequencer following manufacturer's protocol.

cDNA Preparation: Retinas were collected at six separate developmental ages (P1, P7, P13, P21, P26 and 3 Months) from $C 57 B L / 6 J$ and $N y x^{\text {nob }}$ mice. For most experiments retinas from 2-3 animals were pooled for each age point. Tissue collection was repeated 4 times to provide biological replicates at each developmental stage. RNA was extracted using the Ribo-pure kit according to the manufacturer's protocol (Ambion, Foster City, CA). RNA was normalized to 50 $\mathrm{ng} / \mu \mathrm{l}$ by measuring the absorbance at $260 \mathrm{~nm}$ using a Nanodrop Spectrophotometer (Thermo Scientific, Waltham, MA). cDNA was then synthesized from total RNA using Superscript III (Invitrogen, Carlsbad, CA) reverse transcriptase with $2.5 \mathrm{ng} / \mu \mathrm{l}$ random primers per manufacturer's protocol. 
PCR Conditions: Full-length CDNAs representing the TRPM1 were amplified from retina isolated from adult $C 57 B / / 6$ J mice using Superscript III (Invitrogen, Carlsbad, CA). PCR reactions were performed using Accuprime DNA Taq polymerase (Invitrogen, Carlsbad, CA). A $20 \mu$ PCR reaction volume contained $1 \mathrm{X}$ Accuprime Buffer, $0.5 \mu \mathrm{l}$ of adult $C 57 \mathrm{BV} / 6 \mathrm{~J}$ retinal $\mathrm{CDNA}$ at a $1: 10$ dilution as template, $200 \mu \mathrm{M}$ of the each primer (refer to Table 1 in Chapter II) and $0.2 \mu \mathrm{I}$ Accuprime Taq was run under the following conditions: denature at $94^{\circ} \mathrm{C}$ for $30 \mathrm{sec}$, anneal at $55-65^{\circ} \mathrm{C}$ gradient for $30 \mathrm{sec}$, extend at $68^{\circ} \mathrm{C}$ for 4 minutes for 32 cycles. $10 \mu$ of the PCR reactions were loaded on a $1 \%$ Agarose Gel and run at 90 Volts for 2 hours before Ethidium Bromide (EtBr) staining. PCR bands were visualized under UV light.

Plasmid Constructs: PCR products were cloned into the pCR-Blunt II-TOPO plasmid following manufacturer's protocol (Invitrogen, Carlsbad; EA), transformed into One Shot TOP10 chemically competent cells (Invitrogen, Carlsbad, CA) and plated onto Circlegrow (MP Biomedicals, Solon, $\mathrm{OH}$ ) agar plates containing $50 \mu \mathrm{g} / \mu \mathrm{l}$ Kanamycin (Sigma Aldrich, Saint Louis, $\mathrm{MO}$ ). After overnight incubation at $37^{\circ} \mathrm{C}$, clones were selected and grown in $2 \mathrm{ml}$ liquid Circlegrow with $50 \mu \mathrm{g} / \mu \mathrm{l}$ Kanamycin overnight at $37^{\circ} \mathrm{C}$ while shaking. Plasmid DNA was isolated using a Wizard MiniPrep Kit (Promega, Madison, WI) per manufacturer's instructions and restriction digested to identify positive insertion and digested with Xhol (Promega, Madison, WI). Digested products were separated on an agarose gel (1\% agarose in TBE) at $90 \mathrm{~V}$ for 1.5 hours. Clones containing inserts of the expected size were sequenced in both directions using the following primers (shown $5^{\prime}-3^{\prime}$ ): M13F: GTAAAACGACGGCCAG; M13R: CAGGAAACAGCTATGAC; mTRPM1-005: TGCAAGGCTCACAATGTAGG, mTRPM1-006: CTTTGAGATACTCCAGGACGA, mTRPM1-007: TGCAGAAGATCAACACAAGG, mTRPM1-008: ATGCTGGATGCTCTTGTCCT, mTRPM1-009: TCGGGATGGTGAGGAAATGC， mTRPM1-010: 
CGAGAGCGAGCTGGTGGATGA， mTRPM1-011: CTGCTCATCGTGCTTGTAAG， mTRPM1-012:

TCTACGCCATGGAAATCAACC, mTRPM1-013: TCAACAGTGCCGACGGCTAC, mTRPM1-014: AGACTTGGTGCAGCTTCCGT, mTRPM1-020: CTTGGGCTACTTGCTGCTGT, mTRPM1-026: TGGCCCGAAACATCTTCTAC and mTRPM1-027: AGCCTCTTGCCTTCCTCATC. Sequences were aligned to murine Ensembl TRPM1 sequence (TRPM1; NM_018752) and analyzed using Seqman software (DNAStar).

Strep-tag II (5'-TGGAGCCACCCGCAGTTCGAAAAG-3') tag inserted between the nyctalopin signal sequence and YFP-nyctalopin. For mammilian expression, strep-XFP-nyctatopin (SYFP-NYX)-was inserted into pcDNA3.1+ vector (Invitrogen, Carlsbad, CA) at the EcoR1 site using infusion cloning techniques (Clonetech, Mountain View, CA). For expression of TRPM1 into HEK293T cells a flag-probe (5'-GACTACAAGGACGACGAC-3') was fused to the $5^{\prime}$ end of full length TRPMI CDNA. For stable expression of TRPM1 into HEK293T cells a HA-probe (5'TACCCATACGATGTTCCAGATTACGCT-3') was fused to the $5^{\prime}$ end of full length TRPM1. Final constructs were verified by sequencing.

Quantitative Real-Time PCR: Taqman primers and probes were designed to identify three forms of TRPM1 (Table-6). All isoforms of FRPM1 contain exon 3 and exon 4 , so total TRPM1 (TRPM1T) primers were generated to the 3 and 4 exon junction. The assay for the long forms of TRPM1 (TRPM1-LF) were designed to the 20 and 21 exon junction. The assay for the TRPM1-SV isoform was designed to the 19 and 21 exon junction. Each sample represents an average of three qRTPCR reactions. qRT-PCR was performed in a $10 \mu \mathrm{l}$ reaction volume with $0.5 \mu \mathrm{l}$ of cDNA and the Taqman Master Mix 2.0 according to manufacturer's protocol (Applied Biosystems, Foster City, CA). Thermal cycling and fluorescent detection was performed on a 7900HT Fast Real-Time PCR System (Applied Biosystems, Foster City, CA). 
Table 6. QRT-PCR Taqman primers and probes for TRPM1 splice variants in murine retina

\begin{tabular}{llll}
\hline PCR & Forward Primer & Probe & Reverse Primer \\
\hline TRPM1-T & CCTTCAAGCGTGGTTCAATCA & AGCTCCACATCAGgG TCCCAGAAGG & TGTCTTCTCTATCCATGCTTCTGA \\
TRPM1-LF & CATTGGAACAAAG ATCTGTGAATTCT & CGCCCATTGTCAAGTTCTGGTTITACACA & ACAGCAGCAAGTAGCCCAAGTAG \\
TRPM1-SV & GATGACTCTCCTATCAAACATCCAA & AAGATG GCAAAGAAAAGGAAGAAGAGAATGTGATIT & ACAGCAGCAAG TAGCCCAAG TAG \\
\hline
\end{tabular}

The concentration of TRPM1 variant in each sample was determined using a standard curve generated by serial dilutions of TOPO-TRPM1-LF and TOPO-TRPM1-SV plasmid. TRPM1-T slope of the curve was calculated as $y=-3.5288 x+21.311$, TRPM1-LF was $y=-3.2997 x+22.37$ and TRPM1-S $\forall$ was $y=-3.6381 x+21.796$ where $y$ is the eycle number $(C t)$-and $x$ is the log of the pg of RNA (log[pg]). The concentration for each variant was normalized to adjust for input cDNA using a Taqman assay for 18S RNA (Applied Biosystems, Foster City, CA).

Retinal Preparation: Normal human eyes were obtained from Kentucky Lions Eye Bank. For transverse retina sections: freshly dissected retinas were fixed for 10 minutes in $4 \%(w / v)$ paraformaldehyde (Sigma Aldrich, Saint Louis, MO) in $0.1 \mathrm{M}$ phosphate buffer (PB). They were then washed in $0.1 \mathrm{M}$ PB before being cryoprotected through a graded sucrose series $(5 \%, 10 \%$,


cut on a cryostat (Leica Microsystems, Bannockburn, IL), and mounted onto superfrost glass slides (Fisher, Waltham, MA). For whole mount retinas: freshly dissected retinas were fixed for 5 minutes in $4 \%(\mathrm{w} / \mathrm{v})$ paraformaldehyde in $0.1 \mathrm{M}$ phosphate buffer $(\mathrm{PB})$. They were then washed in 0.1M PB before being cut into quarters. Retinal quarters were mounted photoreceptor side down on nitrocellulose membrane. 
Immunohistochemistry: Frozen sections were brought to room temperature (RT), washed in PBS for $5 \mathrm{~min}, 0.5 \%(\mathrm{v} / \mathrm{v})$ Triton $\mathrm{X}-100$ in PBS for 5 minutes and then incubated in blocking solution containing: $5 \%(\mathrm{v} / \mathrm{v})$ normal goat serum, $0.5 \%(\mathrm{v} / \mathrm{v})$ Triton $\mathrm{X}-100$ in PBS for $1 \mathrm{~h}$. Primary antibodies were diluted in blocking solution and incubated at RT overnight. Antibodies and their dilutions are listed in Table 7. After incubation with the primary antibody, sections were washed in PBS $3 X$ for 5 minutes each, sections then were incubated in fluorescent secondary antibodies (1:1000 in blocking solution) at RT for $1 \mathrm{~h}$. Secondary antibodies were Alexa 488 goat anti-rabbit; Alexa 555 goat anti-mouse; Alexa 568 donkey anti-rabbit (Invitrogen, Carlsbad, CA). Alexa-643labeled PNA (Invitrogen, Carlsbad, CA, at 1:1000) was added with secondary antibodies. Slides were then washed 3X in PBS and coverslipped with Immunomount (ThermoShandon, Waltham, MA). Immunostained sections were imaged on an Olympus FV300 confocal microscope, using a $60 x$ oil objective (1.45 NA). Images shown are maximum projections of confocal stacks, adjusted for contrast and brightness with Fluoview software.

Table 7. Primary antibodies used for immunohistochemistry.

\begin{tabular}{cclll}
\hline Antibody & Dilution & Company & Species & Antigen \\
\hline TRPM1 & $1: 100$ & Sigma (HPAC1 4785) & Rabbit & Synthetic peptide from C-terminus of human TRPM1 (1449-1567aa) \\
TRPM1 & $1: 100$ & Osaka Bioscience institute & Rabbit & Synthetic peptide from C-terminus of mouse TRPM1 (1554-1622aa) \\
RiBEYE & $1: 1000$ & BD Biosciences (612044) & Mouse & Synthetic peptide from mouse CtBP2 (361-445aa) \\
GOa & $1: 1000$ & Chemicon (MAB3073) & Mouse & Purified bovine brain GOa \\
Grm6 & $1: 1000$ & Neuromics (RA13105) & Rabbit & Synthetic peptide from C-terminus of rat mGluR6 (AAPPQNENAEDAK) \\
GFP & $1: 1000$ & Imvitrogen (A11120) & Mouse & Full length GFP \\
GFP & $1: 1000$ & Irwitrogen (A21311) & Rabbit & Full length GFP antibody conjugated to Alexa-488 \\
\hline
\end{tabular}

Quantitative analysis of immunohisochemistry from confocal images was performed using the Fluoview software package-(Olympus, Center Valley, PA). TRPM1 images co-tabeled 
with RIBEYE, PNA, or YFP-nyctalopin were selected for further analysis. The region of interest, the OPL, was determined by the positive staining for the marker protein and used as a reference for the normalization of the recorded fluorescence intensity in the channel containing protein being measured. The fluorescence intensity within synaptic puncta was analyzed using line-scan mode. A $2 \mu \mathrm{m}$ long line was placed across individual punctum using PNA as a reference or $2.5 \mu \mathrm{m}$-long line was placed across the ribbon using RIBEYE as a reference. The distribution of the maximal intensities along this line was determined in both channels and averaged across 10 individual lines. In human retina, the fluorescence intensity between the two channels was equal, so reported as pixel versus distance. In mouse retina, the TRPM1 intensity was decreased compared to the markers, so the fluorescence was normalized to the peak and reported as normalized versus distance. The intensity distribution in each channel was plotted and analyzed using Microsoft Excel software.

Cell Culture: HEK293T (human embryonic kidney cells; ATCC, Manassas, VA) cells were grown in high glucose DMEM supplemented with $10 \%$ fetal bovine serum, L-glutamine ( $2 \mathrm{mM}$ ), penicillin $(50 \mathrm{lU} / \mathrm{ml})$ and streptomycin $(50 \mu \mathrm{g} / \mathrm{ml})$. Transient transfections were performed using CalPhos Mammalian Transfection Kit (Clonetech, Mountain View, CA) according to the manufacturer's protocol.

The PCDNA3.1:HA-TRPM1 vector was linearized with $M f e l$ and purified using the QIAQuick PCR Purification Kit (Qiagen, Valencia, CA). Stable cell lines expressing HA-TRPM1 were produced by transfecting cells using the CalPhos Mammalian Transfection Kit (Clonetech, Mountain View, CA) according to the manufacturer's protocol. Next day, HA-TRPM1 transfected HEK293T cells were diluted 1:100 and grown under $\mathrm{G} 418(1 \mathrm{mg} / \mathrm{ml})$ selection for four weeks. Single colonies were 
picked, grown to confluence and then screened for TRPM1 activity by whole-cell patch clamp recordings.

Strep-tactin Magnetic Bead Pull Down: Two $10 \mathrm{~cm}$ plates of transiently transfected HEK293T cells were lysed by sonicating in $400 \mu \mathrm{lN}-\mathrm{T}$ Lysis buffer (NaH2PO4, NaCl, Tween-20, pH to 8.0 with $1 \mathrm{M} \mathrm{NaOH}$ ). The homogenate was cleared by centrifuging at $15,000 \times g$ for 10 minutes at $4^{\circ} \mathrm{C} .40 \mu \mathrm{l}$ of total lysate was saved, while the remaining was incubated with $200 \mu \mathrm{l}$ of streptactin magnetic beads (Qiagen, Valencia, CA) on a rocker at $4^{\circ} \mathrm{C}$ for $2-3 \mathrm{hrs} .40 \mu \mathrm{l}$ from the flow through fraction was collected and saved. After three washes with the NP-T lysis buffer, the proteins were eluted from the beads with $10 \mathrm{mM}$ D-Biotin (Sigma Aldrich, Saint Louis, MO), in the same NP-T lysis buffer. SDS sample buffer (62 mM Tris, $10 \%$ glycerol, $2 \%$ SDS, and $5 \% \beta$ mercaptoethanol) was added and samples were incubated for 10 minutes at $70^{\circ} \mathrm{C}$ before loading the $4-12 \%$ NuPAGE gel (Invitrogen, Carlsbad, CA).

Membrane Fractionation: Three $10 \mathrm{~cm}$ plates HEK293T cells or retinas from 4 mice $\left(\mathrm{Ny} \mathrm{x}^{\text {nob }}\right.$ and TgYFPNYX) were collected and washed in $1 X$ PBS before resuspending in hypotonic swelling buffer (10 mM Tris, $15 \mathrm{mM} \mathrm{KCl,} 1 \mathrm{mM} \mathrm{MgCl}$, pH7.5 with $1 \times$ Complete Protease Inhibitor, Roche, Basel, Schweiz). The homogenate was incubated on ice for 15 minutes before adjusting the buffer to contain $250 \mathrm{mM}$ Mannitol and $1 \mathrm{mM}$ EDTA. Cells were homogenized with a glass pestle and dounce before clearing by centrifugation at $600 \times g$ for 10 minutes. Membrane fractions were collected at $100,000 \times g$ for 20 minutes at $4^{\circ} \mathrm{C}$. The fraction of the supernatant was saved as the cytosolic fraction. The pelleted membranes were then resuspended in $100 \mu$ l Protein-G buffer $(250 \mathrm{mM}$ Tris- $\mathrm{HCl}, 150 \mathrm{mM} \mathrm{NaCl}, 1 \%$ Non-indet P-40, $0.5 \%$ sodium deoxycholate, $\mathrm{pH} 7.5)$. 
GFP Antibody Immunoprecipitation: For immunoprecipitation retinas from 1 rabbit or at least 3 mice were homogenized in $500 \mu \mathrm{l}$ extraction buffer. Three separate buffers were tested. Protein-G buffer $(50 \mathrm{mM}$ Tris- $\mathrm{HCl}, \mathrm{pH} 7.5,150 \mathrm{mM} \mathrm{NaCl}, 0.1 \%$ Nonidet $\mathrm{P}-40,0.05 \%$ sodium deoxycholate, $1 X$ complete protease inhibitor, Roche, Basel, Schweiz); Brandstätter Buffer (50 $\mathrm{mM}$ Tris- $\mathrm{HCl}, \mathrm{pH} 7.5,150 \mathrm{mM} \mathrm{NaCl}, 1 \%$ Triton $\mathrm{X}-100,0.5 \%$ sodium deoxycholate, $1 \mathrm{X}$ complete protease inhibitor; tom Dieck et al, 2005); Morgans Buffer ( $1 \%$ NP-40, $0.5 \%$ sodium deoxycholate, 0.1\% SDS, $1 \mathrm{mM}$ EDTA, $150 \mathrm{mM} \mathrm{NaCl}$, 50-mM Tris; pH 8.0, 1X complete protease inhibitor; Morgans et al., 2005). The homogenates were subjected to centrifugation at $14,000 \mathrm{X}$ $g$ for 10 minutes at $4^{\circ} \mathrm{C}$ to remove insoluble material.

For co-immunoprecipitation experiments from transfected cells, HEK293T cells from two $10 \mathrm{~cm}$ plates were lysed by sonicating in $400 \mu$ l Protein-G Buffer $(50 \mathrm{mM}$ Tris- $\mathrm{HCl}, \mathrm{pH} 7.5,150$ $\mathrm{mM} \mathrm{NaCl}, 0.1 \%$ Nonidet $\mathrm{P}-40,0.05 \%$ sodium deoxycholate, $1 \mathrm{X}$ complete protease inhibitor, Roche, Basel, Schweiz). The homogenate was centrifuged at $14,000 \times g$ for 10 minutes at $4^{\circ} \mathrm{C}$.

Total lysate $(40 \mu \mathrm{l})$ from either retina or HEK293T cells was saved, while the remaining was incubated with $1 \mu \mathrm{g}$ GFP antibody (MBL International, Woburn, MA) rotating at $4^{\circ} \mathrm{C}$ overnight. Next day, $40 \mu$ l Protein-G agarose beads (Roche, Basel, Schweiz) were added and incubated for 2 hours at $4^{\circ} \mathrm{C} .40 \mu \mathrm{l}$ from the flow through fraction was collected and saved. Beads were washed $2 X$ in wash buffer $2(50 \mathrm{mM}$ Tris- $\mathrm{HCl}, \mathrm{pH} 7.5,500 \mathrm{mM} \mathrm{NaCl}, 0.1 \%$ Nonidet $\mathrm{P}$ 40 and $0.05 \%$ sodium deoxycholate) and $2 \mathrm{X}$ in wash buffer $3(10 \mathrm{mM}$ Tris- $\mathrm{HCl}, \mathrm{pH} 7.5,0.1 \%$ Nonidet P-40 and $0.05 \%$ sodium deoxycholate). Elution off the GFP antibody was preformed in $40 \mu$ Protein-G buffer with $5 \times$ SDS sample buffer at $70^{\circ} \mathrm{C}$ for $10 \mathrm{~min}$. 
Deglycosylation: Retina protein was extracted into $4 \mathrm{M}$ guanidine $\mathrm{HCL}, 10 \mathrm{mM}$ Sodium Acetate and $10 \mathrm{mM}$ EDTA. Lysates were then concentrated using Centricon YM-3 filters and diluted into the appropriate glycoslyation buffer (Table 8). After the reaction, protein was TCA precipitated, resuspended in SDS sample buffer and loaded onto an SDS-PAGE gel. 10 $\mathrm{gg}$ Bovine Fetuin (Sigma, Saint Louis, MO) was used as a control in Neurominidase experiments. Fetuin control samples were resolved on SDS-PAGE and visualized by coomassie blue staining.

Table 8. Glycosidase buffers and reaction conditions

\begin{tabular}{|c|c|c|c|}
\hline Ghycosidase & Company & Buffer/Protocol & Reaction \\
\hline O-Glycosidase & Simp (G 1163) & 5X Reaction Buffer -250mM Sodium Phosphate, pH 5.0 & 3 hours at $37^{\circ} \mathrm{C}$ \\
\hline Neurominidase & Sigma (N3786) & 50 mM Sodium Phosphate, $\mathrm{pH} 60$ & 3 hours at $37^{\circ} \mathrm{C}$ \\
\hline Chondrotinase ABC & Sigma (C 2905) & $250 \mathrm{mM}$ Tris, pH8.0, $300 \mathrm{mM}$ Sodium Acetate, $0.5 \%$ BSA & 3 hours at $37^{\circ} \mathrm{C}$ \\
\hline Hyalur onidase & $\begin{array}{l}\text { Atlanta } \\
\text { Biochemicals }\end{array}$ & $500 \mathrm{mM}$ Sodium Phosphate with $150 \mathrm{mM}$ Sodium Chioride, pH 5.3 & 3 hours at $37^{\circ} \mathrm{C}$ \\
\hline Collagenase Type 2 & $\begin{array}{l}\text { Worthington } \\
\text { Biochemical }\end{array}$ & $0.05 \mathrm{M}$ TES with $0.36 \mathrm{mM}$ Calcium Chloride, $\mathrm{pH} 7.5$ & 5 hours at $37^{\circ} \mathrm{C}$ \\
\hline PNGase F & Sigma (P 7367) & $50 \mathrm{mM}$ Sodium Phosphate, $\mathrm{pH} 7.5$ & 3 hours at $37^{\circ} \mathrm{C}$ \\
\hline Endoghrosidase F1 & Sigma (E 9762) & $250 \mathrm{mM}$ Sodium Phosphate, pH 5.5 & 1 hour at $37^{\circ} \mathrm{C}$ \\
\hline Endoghrcosidase $F 2$ & Sigma (E O639) & $250 \mathrm{mM}$ Sodium Acetate, pH 4.5 & 1 hour at $37^{\circ} \mathrm{C}$ \\
\hline Endoghrosidase F3 & Sigma (E 2264) & $250 \mathrm{mM}$ Sodium Acetate, $\mathrm{pH} 4.5$ & 1 hour at $37^{\circ} \mathrm{C}$ \\
\hline
\end{tabular}


Western Blotting: Protein fractions were analyzed on 4-12\% NuPAGE gels (Invitrogen, Carlsbad, CA) following the manufacturers protocol. Protein bands were transferred onto PVDF membranes (GE Healthcare, Piscataway, NJ). Immunoblot analysis was performed using primary antibodies at the specific dilutions as listed in Table 9. Anti-mouse (Sigma Aldrich, Saint Louis, MO) and anti-Rabbit (Pierce, Rockford, IL) HRP-conjugated secondary antibodies were used to detect bands with the advanced ECL (Pierce, Rockford, IL) detection system.

Table 9. Primary antibodies used for immunoprecipitation and western blot.

\begin{tabular}{llclll}
\hline Antbody & Company & Dilution & Species & Method & Antigen \\
\hline HA-probe F-7 & Sigma (sc-7392) & $1: 500$ & Rabbit & WB & Hemaggiutinin amino acids 90-115 \\
Flag & Sigma (F3165) & $1: 2000$ & Mouse & WB & Flag M2 (DKKDDDDK) \\
GFP & MBL (M048-3) & 1 1.g & Mouse & iP & Full length GFP protein \\
GFP & Cell Signaling (2555) & $1: 2000$ & Rabbit & WB & Synthetic GFP peptide coupled to KLH \\
Grm6 & Gift from Dr. Massey & $1: 1000$ & Rabbit & WB & Synthetic peptide from C-Terminus of Rabbit Grm6 \\
\hline
\end{tabular}

Immunocytochemistry: HEK293T cells $\left(4 \times 10^{5}\right.$ cells) were plated onto poly-lysine coated coverslips. The next day cells were transiently transfected using the CalPhos transfection system per the manufacturer's protocol (Clonetech, Mountain View, CA) with $1 \mu \mathrm{g}$ of pcDNA3.1:SYFPNYX, pcDNA3.1:F-Grm6, or pcDNA3.1:FG-TRPM1 per coverslip. After 24 hours, cells were fixed for 5 minutes in $100 \%$ Methanol at $-20^{\circ} \mathrm{C}$ then for 5 minutes in $1: 1$ Methanol/Acetone at $-20^{\circ} \mathrm{C}$ followed by three washes in $1 \times$ PBS. Fixed cells were then incubated with primary antibody diluted to $1: 500$ in a $3 \%$ BSA blocking solution in 1XPBST (0.1\% Tween-20 in 1 X PBS) for 1 hour at room temperature. A list of primary antibodies is shown in Table 10. The coverslips were washed in 1 X PBST before incubating with secondary Alexa-488 goat anti-rabbit, Alexa- 555 goat 
anti-mouse, Alexa-568 donkey anti-rabbit (Invitrogen, Carlsbad, CA) at a 1:500 dilution in blocking solution (3\% BSA blocking in 1XPBST) for 1 hour at room temperature. Coverslips were washed with $1 \times$ PBST and mounted onto permafrost slides using Immunomount (ThermoShandon, Waltham, MA) then sealed with nail polish. Immunostained cells were imaged on an Olympus FV300 confocal microscope, using a $60 \mathrm{X}$ oil objective. Images shown are maximum projections of ten $0.5 \mu \mathrm{m}$ confocal stacks, adjusted for contrast and brightness with Fluoview software.

Table 10. Primary antibodies used for immunocytochemistry.

\begin{tabular}{llll}
\hline Antibody & Company & Species & Antigen - \\
\hline Na/K ATPase & Sigma & Mouse & Synthetic peptide from 646-652aa of Sheep kadney a subunit \\
Calnexin & BO Biosciences & Mouse & Synthetic peptide from 116-301aa of Human Calnexin \\
Flag & Sigma & Mouse & Synthetic peptide OKKDOODKC \\
GFP & Imvitrogen (A21311) & Rabbit & GFP antibody conjugated to Alexa-488 \\
\hline
\end{tabular}

Patch Clamp Recordings: For whole-cell recordings, HEK293T or HA-TRPM1 stable cells were grown on coverslips then transferred to standard external solution. Patch pipettes were fabricated from borosilicate glass (WPI, Sarasota, FI) and filled with a $\mathrm{K}^{+}$-based solution with 5.5 mM EGTA, $10 \mathrm{mM}$ HEPES and $4 \mathrm{mM}$ ATP (pH 7.25 by KOH). The TRP channel agonist Capsaicin (20 $\mu \mathrm{M}$, Sigma Aldrich, Saint Louis, MO) and antagonist Capsazepine (20 $\mu \mathrm{M}$, Sigma Aldrich, Saint Louis, MOH were delivered to the cells from a pipette using positive pressure (2-4 PSI) with a computer-controlled solenoid valve (Picospritzer, General Valve Corp). The holding potential for all recordings was $+50 \mathrm{mV}$. 
Recording and analysis: Whole-cell recordings were obtained with an Axopatch 1D amplifier (Molecular Devices, Sunnyvale, CA). Currents were acquired at a sampling rate of $2 \mathrm{kHz}$ with Axograph X software and an Apple G5 computer, low pass filtered at $50 \mathrm{~Hz}$ (Frequency Devices, Ottawa, IL) and-digitized with an ITC-18 interface (Heka, BeHmore, NY). Holding potentials were corrected for the liquid junction potential, which was measured to be $10 \mathrm{mV}$ with the standard $\mathrm{K}^{+}$gluconate pipette solution. Recordings were discarded if the series resistance exceeded 20

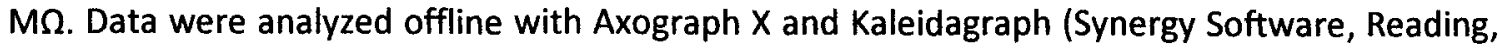
PA). The student $t$-Test was used to analyze the data. 


\section{CHAPTER VI: DISCUSSION}

My data show that the TRPM1 channel is expressed in the DBCs where it co-localizes with nyctalopin at the dendritic tips. Proper localization of TRPM1 to the dendritic tips requires expression of nyctalopin and Grm6. In addition, using a heterologous expression system I found that TRPM1 interacts with nyctalopin. I interpret these results to indicate that nyctalopin plays a role in localizing the TRPM1 channel in the dendritic tips of the DBCs. Whether nyctalopin is required for trafficking to the membrane, stabilizing or clustering the channel in the membrane or assembling channel subunits remains to be determined.

\section{Why are there Multiple TRPM1 Splice Variants in the Retina?}

Alternative splicing occurs in all eukaryotes, and in humans over $80 \%$ of genes are believed to be alternatively spliced, thus greatly increasing the diversity of proteins encoded by the genome (Matlin et al., 2005). I identified five splice variants of TRPM1 from retina CDNA.

In the retina, splice variants can have functions in the same cell or be expressed in different cells. The splice variants of the G-protein heterotrimer subunits $G \alpha_{0}: G \alpha_{01}$ and $G \alpha_{02}$ (Goldsmith et al., 1988; Strathmann et al., 1990), are present in the DBCs (Dhingra et al., 2000, 2002). The highly abundant $\mathrm{Ga}_{\mathrm{O} 1}$ variant is required for the light-evoked activity of the $\mathrm{DBCS}$ (Dhingra et al., 2002); however, a recent report also showed that $G \alpha_{02}$ plays a role in generating the $\mathrm{DBC}$ response though 10-fold less sensitive than $\mathrm{Ga}_{01}$ (Pahlberg et al., 2009). The $\mathrm{Ga}_{\mathrm{o}}$ splice variants work together to improve the light sensitivity in the DBCs.

The $\beta$-subunit of the trimeric G-protein involved in Grm6 function also exists as two splice variants, G $\beta 5-L$ and $G \beta 5-S$, which are found in two different cell types within the retina. 
Both splice variants interact with and stabilize the RGS proteins: RGS6, RGS7, RGS9, and RGS11. The photoreceptor-specific long form, GB5-L, interacts with RGS9 and R9AP to confer intracellular stability, membrane affinity, and outer segment targeting modality to the G $\beta 5-L$, RGS9, R9AP complex (Chen et al., 2003; Martemyanov et al., 2003; Keresztes et al., 2004). This complex regulates the intrinsic GTPase activity of transducin, $\mathrm{G} \alpha_{\mathrm{t}}$ (He et al., 1998; Makino et al., 1999). The short form, GB5-S, is found in the DBCs where it co-localizes with RGS7 and RGS11 and is required for proper DBC responses (Morgans et al., 2007; Rao et al., 2007). The G $\beta 5$ splice variants are involved in terminating the G-protein cascade in both photoreceptors and DBCs (Rao et al., 2007).

My analyses of the TRPM1 splice variant expression profile during development suggests the variants with all six transmembrane domains are expressed in bipolar cells. Morphological classification of DBCs have identified 6-8 cell types in the mammalian retina (Boycott \& Wässle, 1991; Euler \& Wässle, 1995; Ghosh et al., 2004); however, little is known about the functional difference between each type. Each DBCs type could be expressing a different TRPM1 variant that might contribute to different kinetics in their light-evoked responses. Furthermore, my analysis showed that the TRPM1 variant lacking transmembrane domain 2 (TRPM1-SV) had a temporal pattern during development more similar to PR expression. Therefore this variant could have a function in the PRs.

Apart from each TRPM1 isoform being expressed to a specific DBC type they could work in combination in all DBCs. Endogenous TRPM1 expressed in B16-F10 epidermal melanocytes or human melanoma cells have high outward rectification (Oancea et al., 2009). In comparison the current-voltage relationship in DBCs exhibits a moderate outward rectification (Shen et al., 2009). The difference in the biophysical properties of the TRPM 1 channel between these separate systems may reflect differences in the TRPM1 splice variant composition. Furthermore, 
TRP channels are known to be hetero-tetrameric channels with other TRP members. The moderate outwardly rectifying current seen in DBCs could also reflect a heterotetrameric channel between TRPM1 and another TRP channel.

Further analysis of the retinal TRPM1 splice variants could be determined in a heterologous system. Recombinant expression of each variant followed by calcium imaging or patch clamp recordings could be used to determine if they form a functional cation channel. Studies were performed for TRPM1 variants identified in human melanocytes (Oancea et al., 2009). Of the four N-terminal TRPM1 variants they identified, all contained six transmembrane domains, yet only two of the variants displayed currents similar to full-length TRPM 1 when expressed in a heterologous system. This suggests that some of the retinal splice variants may not even form a functional cation channel.

\section{TRPM1 Instability in the DBC Dendritic Tips}

The first TRP channel identified was found in Drosophila and is required for phototransduction (Montell \& Rubin 1989). This finding has served as a standard model of macromolecular organization of TRP channels. The Drosophila TRP channel associates with other signaling proteins to form a large macromolecular complex, the signalplex. INAD (Inactivation No After Potential D) is a PDZ scaffold protein that is a central player in the signalplex. Interactions between TRP and INAD are required to target the complex to the rhabdomere membrane (Chevesich et al. 1997). To determine if a similar signaling complex was formed in DBCs, I investigated the interdependence of TRPM1, Grm6 and nyctalopin expression. A summary of the immunohistochemical results from wildtype and three knockout mice, TRPM ${ }^{\prime-}$, $G r m 6^{n o b 4}$ and $N y x^{n o b}$ are displayed in Table 11 and Figure 33. 
Table 11. Expression of PR and DBC proteins in the OPL of CSNB1 mutant mice Genotype

\begin{tabular}{|c|c|c|c|c|}
\hline Protein & C57BI/6J & $N y x^{n o b}$ & TRPM1\% & Grm6 nabs \\
\hline Nyctalopin & $t^{\circ}$ & $*$ & + & + \\
\hline TRPM1 & $t^{b}$ & - & $-^{b}$ & - \\
\hline Grm6 & $t^{c}$ & $t^{\circ}$ & $t^{b}$ & - \\
\hline
\end{tabular}

(a) Gregg et al., 2003; (b) Morgans et al., 2009; (c) Pinto et al., 2007

- No antibody for nyctalopin

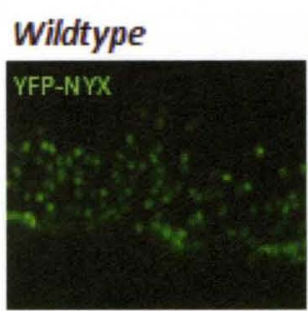

$N y x^{n o b}$
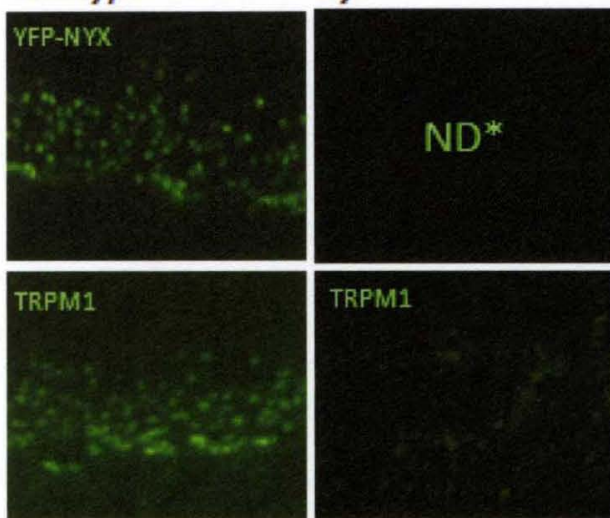

GRM6
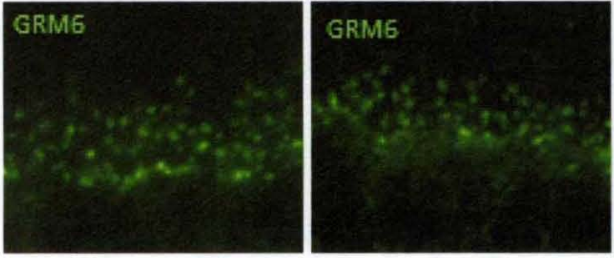

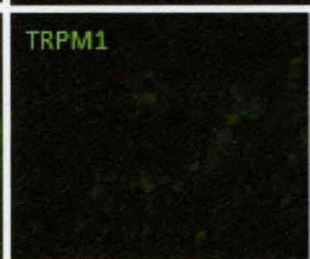

GRMG
TRPM1 $^{\%}$

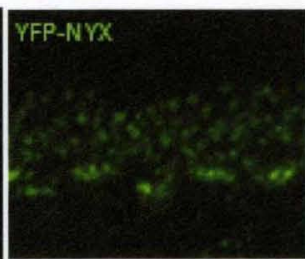

TRPM1

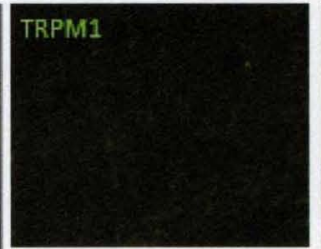

GRM6

5.

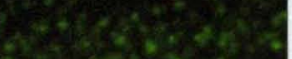

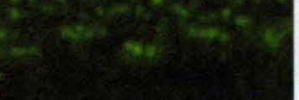

Grm6 nob4
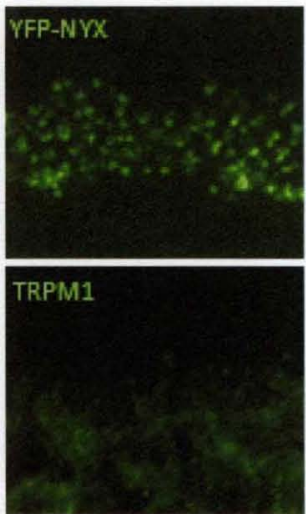

\section{GRM6}

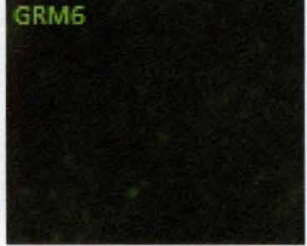

Figure 33. Summary of immunohistochemical staining of DBC membrane proteins from three CSNB1 mutant mice compared to wildtype. Antigen stained is indicated in each panel. *Not determined antibody for nyctalopin.

In the Nyx ${ }^{\text {nob }}$ mice TRPM1 is absent from the OPL; however, TRPM1 remains in the cell bodies of the DBCs in the INL. This suggests that TRPM1 is still expressed but is not being properly transported or clustered to the tips of the dendrites. To further determine if TRPM1 is in the membrane our collaborator, Dr. Nawy, performed patch clamp recordings from $N y x^{\text {nob }}$ rod BCs. 
Figure 34 shows the responses from wildtype and $N y x^{\text {nob }}$ rod $B C s$ to application of agonists. LY341495 inhibits Grm6 and generates an outward current (Figure 34A, Snellman et al., 2008). The application of LY341495 does not elicit a current in the $N y x^{\text {nob }}$ mice (Figure 34A). To directly stimulate TRPM1 channels in the membrane the TRPM1 agonist, capsaicin, was puffed onto rod BCs. In wildtype rod BCs capsaicin elicits an outward current similar to LY341495. However, capsaicin was unable to elicit a current in Nyx ${ }^{\text {nob }}$ rod BCs (Figure 34A). These data support that the TRPM1 channel is not present in the membrane of rod BCs in the absence of nyctalopin. I postulate that nyctalopin is likely involved in trafficking or inserting the TRPM1 channel in the DBC dendrites or assembling the tetrameric TRPM1 channel.

A

$$
\text { Wildtype }
$$
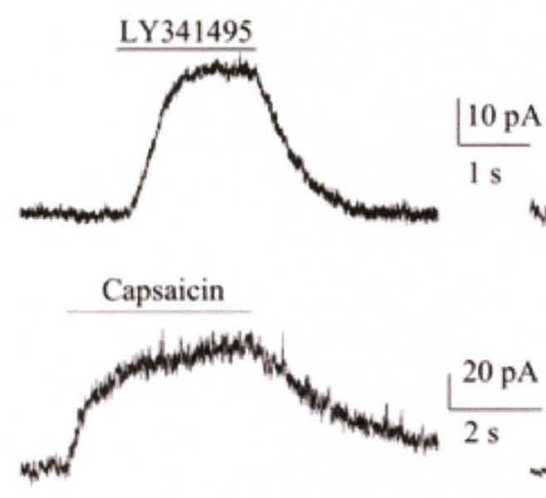

Nyx $x^{\text {nob }}$
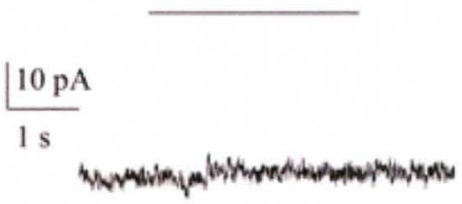

B

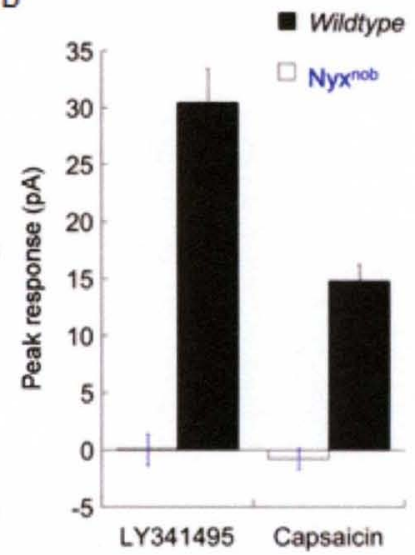

Figure 34. Rod BC patch clamp recordings. (A) Wildtype responds to the Grm6 receptor antagonist, LY341495, with an outward current. This outward current is also elicited by direct stimulation of TRPM1, using the agonist, Capsaicin. Nyx ${ }^{\text {nob }}$ rod BCs do not respond to either LY341495 or capsaicin. The Grm6 cascade is unable to act on TRPM1 because TRPM1 is not in the membrane. (B) Histogram of the peak response for each condition. Patch clamp recordings from Dr. Nawy unpublished data.

In $\mathrm{TRPM}^{-}$retina nyctalopin remains expressed in the dendritic tips of the DBCs, suggesting that nyctalopin localization and stability is not dependent on TRPM1 expression. One possibility for nyctalopin's stability is if it is acting as a scaffold protein. Then nyctalopin could be independently transported to the DBC dendrites where it interacts with extracellular matrix proteins to maintain stability. 
In both TRPM1 and nyctalopin mutants, the Grm6 receptor is properly expressed in the DBC dendrites. These results suggest that Grm6 has an independent means of transport or stabilization in the DBC dendritic membrane.

The Grm6 mutants have nyctalopin present in the DBC dendrites; however, TRPM1 expression in the DBC dendrites decreases though it is expressed in cell bodies in the INL. Interestingly, patch clamp recordings suggest that the TRPM1 channel is constitutively active in the absence of Grm6. Though the DBCs have no response to the TRPM1 channel agonist, capsaicin, the TRPM1 antagonist, capsazepine, is able to produce a small inward current (Figure 36). This suggests the TRPM1 channel is still in the membrane, but at very low levels and this may explain why it is not detected by Immunohistochemistry.

Presumably in the absence of Grm6 the TRPM1 channel is no longer closed by the activation of the G-protein cascade. The unregulated influx of cations through the channel could cause morphological abnormalities, which have been observed in $\mathrm{Grm}^{\text {nob4 }}$ and $\mathrm{Grm}^{/ /}$mice (Cao et al., 2009; Ishii et al., 2009). The loss of TRPM1 from the dendrites of the Grm6 mutants might be a secondary mechanism to help prevent cell death.

In contrast, YFP-nyctalopin is normally expressed in the Grm6 mutants at the light microscopy level. Therefore, nyctalopin maybe expressed in the abnormal dendrites, while TRPM1 is not. These results can be interpreted two ways. First, nyctalopin may require an intermediary protein that is part of the Grm6 cascade to direct insertion of TRPM1 into the membrane. Therefore, in the absence of Grm6 signaling, nyctalopin is trafficked to the membrane but not bringing TRPM1. Alternatively, TRPM1 may interact directly with Grm6 and require complex interactions between both nyctalopin and Grm6 to maintain stability. Initial yeast-two hybrid experiments to examine interactions between TRPM1 and Grm6 were negative 
(Pearring et al., 2009); however, further analysis of protein interactions in an alternative system will be required to confirm this result.

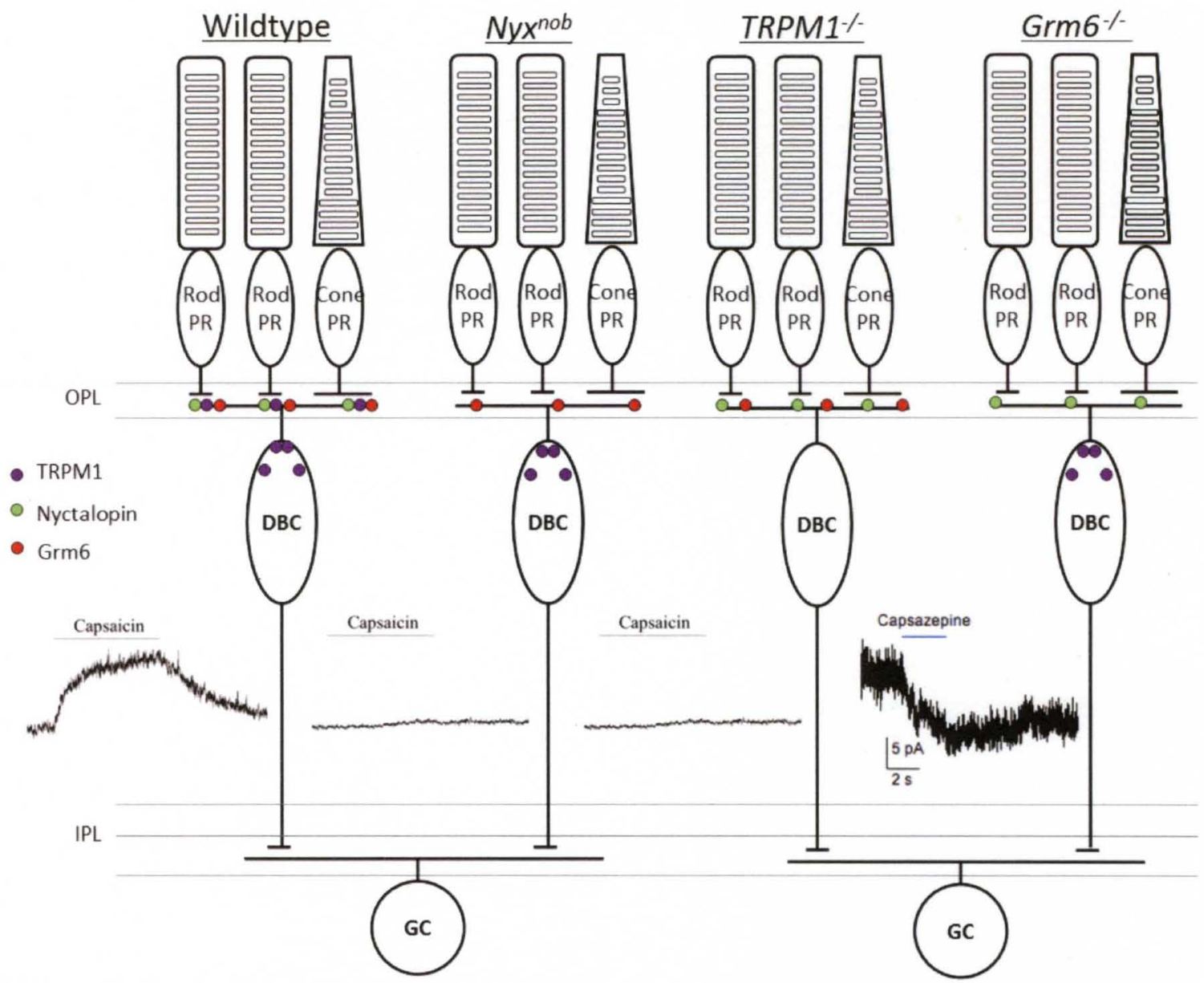

Figure 35. TRPM1 (purple), Nyctalopin (green), and Grm6 (red) protein localization and TRPM1 channel response properties in CSNB1 mouse models: C57BI/6J (wildtype), Nyx ${ }^{\text {nob }}$, TRPM1 $^{-1}$ and Grm6 ${ }^{\prime}$. Currents are from Dr. Nawy unpublished data.

Collectively, these data show that TRPM1 is dependent on both Grm6 and nyctalopin for proper localization. However, nyctalopin and Grm6 expression are not dependent on each other or on TRPM1 (summarized in Figure 35). These results suggest that the three proteins are not forming one large interdependent membrane complex. Furthermore, the instability of TRPM1 in nyctalopin and Grm6 mutants suggests that its expression in the membrane may be activity dependent. Signal-induced translocation to the plasma membrane is a common property of TRP channels (Van Buren et al., 2005; Zhang et al., 2005b; Mehta et al., 2003; Bezzerides et al., 2004; 
Odell et al., 2005; Smyth et al., 2006). Therefore, perhaps signaling through the Grm6 cascade is required for TRPM1 insertion into the membrane. This insertion is not likely to be the gating mechanism for the channel, but could be acting as a secondary means of regulating the amount of TRPM1 in the membrane. Further analysis of TRPM1 expression in the DBC dendrites of other no b-wave mutant mice, such as $G \alpha_{0} 1^{\%}$ or $G 65-S^{\%}$, or CACNA1 $F^{\text {nob2 }}$, would help determine if disrupting Grm6 activity or specific downstream members of the cascade lead to loss of TRPM1 from the DBC dendrites.

\section{Nyctalopin maintains TRPM1 Channel Integrity in the Membrane}

A recent study provided new evidence that TRPM1 may function as an intracellular TRP channel in melanocytes (Oancea et al., 2009). Similar to my own results, localization studies of GFP-tagged TRPM1 isoforms showed they were all localized to intracellular structures (Oancea et al., 2009). This unexpected intracellular location is consistent with the relatively modest magnitude of the whole-cell currents that I recorded from HA-TRPM1 stable cell lines (Chapter IV) and reported in melanocytes (Oancea et al., 2009). In the DBCs, TRPM1 is expressed in the cell bodies as well as the dendritic tips and patch clamp recordings show higher TRPM1 currents suggesting that more TRPM1 channels are expressed in the membrane of these cells (Nawy, 1999; Gregg et al., 2007; Shen et al., 2009). TRPM1 expression in the plasma membrane might require specialized trafficking mechanisms restricted to DBCs. Nyctalopin could be acting to target the TRPM1 channel to the membrane, since it is not expressed in HEK293T cells or melanocytes but restricted to DBCs dendritic tips (Gregg et al., 2007). Both proteins are processed through the ER, so nyctalopin could bind a fraction of the TRPM1 channels to ensure they are placed into specific vesicles bound for the dendritic tips (see trafficking model in Figure 36). Surprisingly, when I transfected nyctalopin into HEK293T cells that stably express TRPM1 the expression of TRPM1 in the membrane was unaltered (Chapter IV). Likely specific neuronal 
proteins or properties are required for TRPM1 and nyctalopin targeting to the plasma membrane.

Another possible role for nyctalopin would be to assemble the TRPM1 channel into tetramers. Interestingly, the structure of nyctalopin is similar to the structure of the ankyrin repeat domain found in many TRP channels (Phelps et al., 2007, 2008). Both are curved structures with $\beta$-sheets on the concave side and helixes on the convex side (Bech-Hansen et al., 2000; Pusch et al., 2000; Gregg et al., 2003; Mosavi et al., 2004; Sedgwick \& Smerdon 1999). Ankyrin repeats are involved in trafficking and assembly of TRP channels in the membrane (Jung et al., 2002; Chang et al., 2004; Erler et al., 2004). Nyctalopin could be acting as an auxiliary ankyrin domain for the TRPM1 channel to ensure it is properly assembled into its tetrameric structure (see assembling model in Figure 36).

Nyctalopin was shown to remain expressed in the DBC dendrites in the absence of both TRPM1 and Grm6. The stability of nyctalopin suggests it maybe binding other proteins, likely extracellular in nature. Nyctalopin interactions maybe providing the proper structure to hold the TRPM1 channel in the membrane and prevent lysosomal degradation of the channel (see stabilizing model in Figure 36).

I showed that nyctalopin interacts with TRPM1 in a heterologous system. Further analysis of nyctalopin's structure could help determine its function with TRPM1. Nyctalopin is an extracellular protein, so it must interact with an extracellular region of TRPM1. To determine where these proteins are interacting, constructs lacking critical protein domains, such as the LRR or TM domain of nyctalopin or specific extracellular loop regions of TRPM1, could be individually tested in the heterologous binding system. In addition, CSNB mutations for nyctalopin could be tested to determine if they interfere with binding. Identifying the specific interacting regions would help to further elucidate the function of nyctalopin. 
TRAFFICKING

ASSEMBLING/STABILIZING

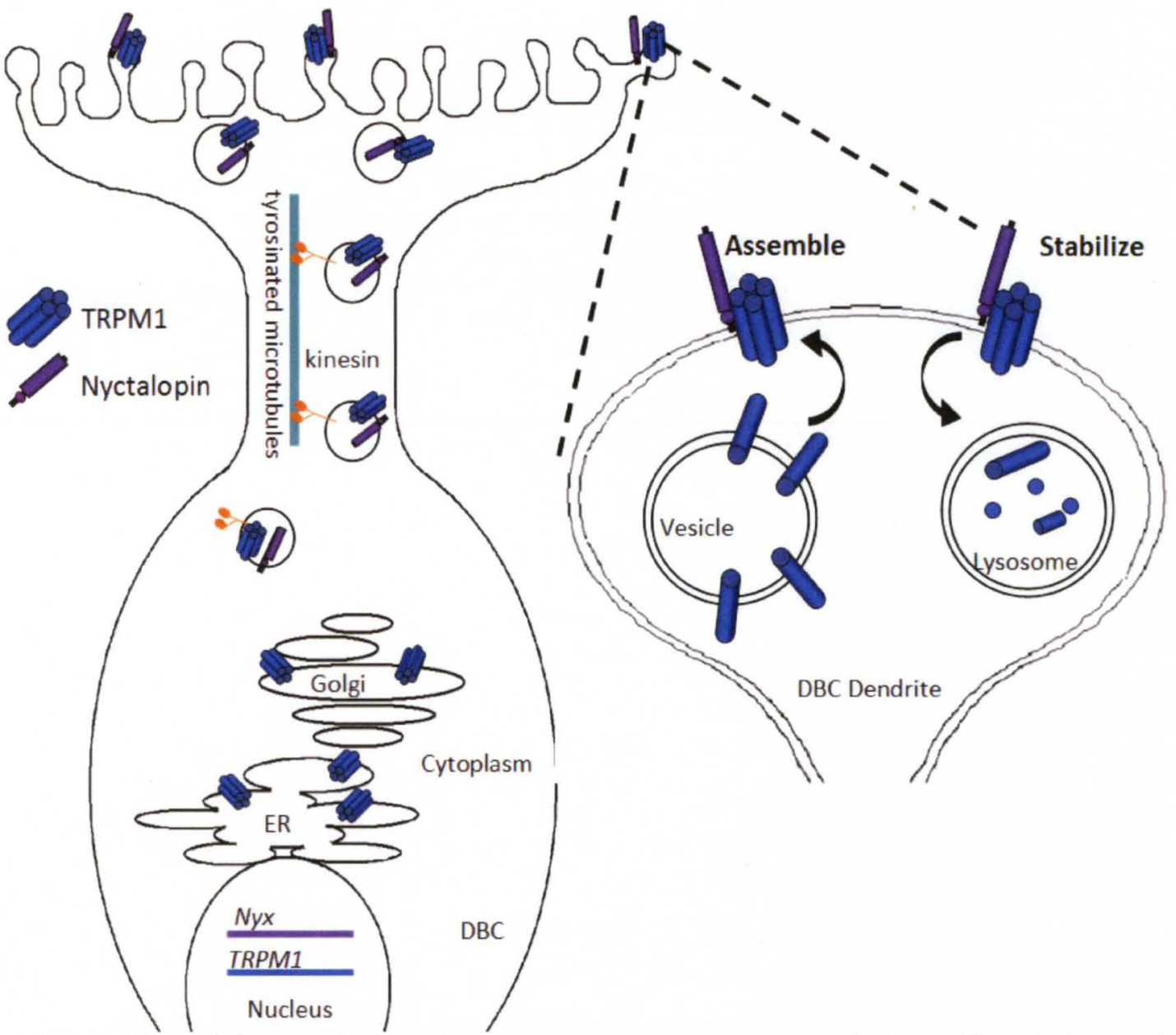

Figure 36. Models for nyctalopin and TRPM1 interactions in DBCs. Nyctalopin could be playing a role in trafficking TRPM1 to the DBC dendritic tips by helping to sequester it into vesicles targeted for dendritic expression. Nyctalopin could be helping maintain TRPM1 stability in the dendritic membrane by being involved in assembly and insertion or by stabilizing it in the membrane to prevent lysosomal degredation.

Since nyctalopin was first identified in 1998, its role in generating the DBC response has remained elusive. My data show that nyctalopin is required for TRPM1 expression in the dendritic tips of DBCs and that it directly interacts with TRPM1. In addition, I characterized how Grm6 impacts TRPM1 localization and provide evidence that nyctalopin is not interacting with Grm6. Collectively my results are the first to reveal the function of nyctalopin in DBCs. 


\section{REFERENCES}

Abramowicz MJ, Ribai P and Cordonnier M. (2005) Congenital stationary night blindness: report of an autosomal recessive family and linkage analysis. Am J Med Genet A 132A:76-79.

Adams H, Scotti PA, Luirink J and Tommassen J. (2002) Defective translocation of a signal sequence mutant in a prlA4 suppressor strain of Escherichia coli. Eur J Biochem 269:55725580.

Adamski FM, Zhu MY, Bahiraei F and Shieh BH. (1998) Interaction of eye protein kinase C and INAD in Drosophila. Localization of binding domains and electrophysiological characterization of a loss of association in transgenic flies. J Bio Chem 273:17713-9.

al Jandal N, Farrar GJ, Kiang AS, Humphries MM, Bannon N, Findlay JB, Humphries $P$ and Kenna PF. (1999) A novel mutation within the rhodopsin gene (Thr-94-lle) causing autosomal dominant congenital stationary night blindness. Hum Mutat 13:75-81.

Ang AL, Taguchi R, Francis S, Folsh H, Murrells $\mathrm{,} \mathrm{Pypaert} \mathrm{M,} \mathrm{Warren} \mathrm{G} \mathrm{and} \mathrm{Mellman} \mathrm{I.} \mathrm{(2004)}$ Recycling endosomes can serve as intermediates during transport from the Golgi to the plasma membrane of MDCK cells. J Cell Biol 167:531-543.

Ahnelt PK, Keri C and Kolb H. (1990) Identification of pedicles of putative blue-sensitive cones in the human retina. J Comp Neurol 293:39-53.

Ahnelt PK, and Kolb H. (1994a) Horizontal cells and cone photoreceptors in primate retina: A Golgi-light microscope study of spectral connectivity. J Comp Neurol 343:387-405.

Ahnelt PK, and Kolb H. (1994b) Horizontal cells and cone photoreceptors in human retina: a Golgi-electron microscopic study of spectral connectivity. J Comp Neurol 343:406-427.

Attwell D, Mobbs P, Tessier-Lavigne M, Wilson M. (1987) Neurotransmitter-induced currents in retinal bipolar cells of the axolotl, Ambrystoma mexicanum. J Physiol 387:125-161.

Audo I, Kohl S, Leroy BP, Munier FL, Guillonneau X, Mohand-Said S, Bujakowska K, Nandrot EF, Lorenz B, Preising M, Kellner U, Renner AB, Bernd A, Antonio A, Moskova-Doumanova V, Lancelot ME, Poloschek Drumare I, Defoort-Dhellemmes S, Wissinger B, Léveillard T, Hamel CP, Schorderet DF, De Baere E, Berger W, Jacobson SG, Zrenner E, Sahel JA, Bhattacharya SS and Zeitz C. (2009) TRPM1 is mutated in patients with autosomal-recessive complete congenital stationary night blindness. Am J Hum Genet. 85:720-729. 
Azzi M, Charest PG, Angers S, Rousseau G, Kohout T, Bouvier M and Pineyro G. (2003) BetaArrestin-mediated activation of MAPK by inverse agonists reveals distinct active conformations for $\mathrm{G}$ protein coupled receptors. Proc Natl Acad Sci 100:11406-11411.

Baas PW, Deitch JS, Black MM and Banker GA. (1988) Polarity orientation of microtubules in hippocampal neurons: uniformity in the axon and nonuniformity in the dendrite. Proc Natl Acad Sci USA 85:8335-8339.

Ball SL, Powers PA, Shin H, Morgans CW, Peachey NS and Gregg RG. (2002) Role of the B2 subunit of voltage-dependent calcium channels in the retinal outer plexiform layer. Invest Ophthalmol Vis Sci 43:1595-1603.

Ball SL, Pardue MT, McCall MA, Gregg RG, and Peachey NS. (2003a) The B2 subunit of voltage dependent calcium channels is required for synaptic transmission in the retinal outer plexiform. Invest Opthalmol Vis Sci 43:1595-1603.

Ball SL, Pardue MT, McCall MA, Gregg RG, and Peachey NS. (2003b) Immunohistochemical analysis of the outer plexiform layer in the nob mouse shows no abnormalities. Vis Neurosci 20:267-272.

Balmforth AJ, Lee AJ, Warburton P, Donnelly D and Ball SG. (1997) The conformational change responsible for AT1 receptor activation is dependent upon two juxtaposed asparagines residues on transmembrane helices III and VII. J Biol Chem 272:4245-4251.

Barthel LK and Raymond PA. (1990) Improved method for obtaining 3-microns cryosections for immunohistochemistry. J Histochem Cytochem 38:1383-1388.

Baylor DA, Fuortes MGF and O'Bryan PM. (1971) Receptive fields of the cones in the retina of the turtle. J. Physiol 214:265-294.

Beavo JA. (1995) Cyclid nucleotide phosphodiesterases: functional implications of multiple isoforms. Physiol Rev 75:725-748.

Bech-Hansen NT, Naylor MJ, Maybaum TA, Pearce WG, Koop B, Fishman GA, Mets M, Musarella MA and Boycott KM. (1998) Loss-of-function mutations in a calcium-channel alpha1-subunit gene in Xp11.23 cause incomplete X-linked congenital stationary night blindness. Nat Genet 19:264-267.

Bech-Hansen NT, Naylor MJ, Maybaum TA, Sparkes RL, Koop B, Birch DG, Bergen AAB, Prinsen CFM, Polomeno RC, Gal A, Drack AV, Musarella MA, Jacobson SG, Young RSL and Welber RG. (2000) Mutations in NYX, encoding the leucine-rich proteoglycan nyctalopin, cause X-linked complete congenital stationary night blindness. Nat Genet 26:319-323.

Bellone RR, Brooks SA, Sandmeyer L, Murphy BA, Forsyth G, Archer S, Bailey E and Grahn B. (2008) Differential gene expression of TRPM1, the potential cause of congenital stationary night blindness (CSNB) and coat spotting patterns (LP) in the appaloosa horse (Equus caballus). Genetics 179:1861-1870. 
Bezzerides VJ, Ramsey IS, Kotecha S, Greka A and Clapham DE. (2004) Rapid vesicular translocation and insertion of TRP channels. Nat Cell Biol 6:709-720.

Blanks JC and Johnson LV. (1983) Selective lectin binding of the developing mouse retina. J Comp Neurol 221:31-41.

Blanks JC and Johnson LV. (1984) Species binding of peanut lectin to a class of retinal photoreceptors. A species comparison. Invest Ophthalmol Vis Sci 25:546-557.

Bojang P and Gregg RG. (2008) Developmental expression of voltage dependent calcium channels (VDCCs) is altered in mice with congenital stationary night blindness $1 \& 2$ (CSNB1\&2). ARVO E-Abstract 1284/A245.

Bojang P, Pearring JN and Gregg RG. (2009) Nyctalopin interacts with transient receptor potential channels in yeast. ARVO E-Abstract 5176.

Bonifacino and Traub. (2003) Signals for sorting of transmembrane proteins to endosomes and lysosomes. Annu Rev Biochem 72:395-447.

Bourne HR. (1986) GTP-binding proteins: One molecular machine can transduce diverse signals. Nature 321:814-816.

Bourne HR and Stryer L. (1992). G proteins: The target sets the tempo. Nature, 358, 541-543.

Boycott BB and Dowling JE. (1969) Retinal ganglion cells: a correlation of anatomical and physiological approaches. UCLA Forum Med Sci 8:145-161.

Buchanan SGSC and Gay NJ. (1996) Structural and functional diversity in the leucine-rich repeat family of proteins. Prog Biophys Mol Biol 65:1-44.

Burack MA, Silverman MA and Banker G. (2000) The role of selective transport in neuronal protein sorting. Neuron 465:472.

Burton PR and Paige JL. (1981) Polarity of axoplasmic microtubules in the olfactory nerve of the frog. Proc Natl Acad Sci 78:3269-3273.

Cajal, S R. (1892) The Structure of the Retina. Madrid. Translated by S.A. Thorpe and M. Glickstein Springfield, II., Thomas, 1972.

Candille SI, Pardue MT, McCall MA, Peachey NS and Gregg RG. (1999) Localization of the mouse nob (no b-wave) gene to the centromeric region of the x-chromosome. IOVS 40:2748-2751.

Cao Y, Song H, Okawa H, Sampath AP, Sokolov M and Martemyanov KA. (2008) Targeting of RGS7/GB5 to the dendritic tips of ON-bipolar cells is independent of its association with membrane anchor R7BP. J Neurosci 28:10443-10449.

Cao Y, Masuho I, Okawa H, Xie K, Asami J, Kammermeier PJ, Maddox DM, Furukawa T, Inoue T, Sampath AP and Martemyanov KA. (2009) Retina-specific GTPase accelerator RGS11/G $\beta$ 
5S/R9AP is a constitutive heterotrimer selectively targeted to mGluR6 in on-bipolar neurons. J Neurosci 29:9301-9313.

Carr RE, Ripps H, Siegel IM and Weale RA. (1966) Visual functions in congenital night blindness. Invest Ophthalmol 5:508-514.

Caterina MJ, Schumacher MA, Tominaga M, Rosen TA, Levine JD and Julius D. (1997) The capsaicin receptor: a heat-activated ion channel in the pain pathway. Nature 389:816-824.

Cepko CL, Austin CP, Yang X, Alexiades M and Ezzeddine D. (1996) Cell fate determination in the vertebrate retina. Proc Natl Acad Sci USA 93:589-595.

Chang B, Heckenlively JR, Bayley PR, Brecha NC, Davisson MT, Hawes NL, Hirano AA, Hurd RE, Ikeda A, Johnson BA, McCall MA, Morgans CW Nusinowitz S, Peachey NS, Rice DS, Vessey KA and Gregg RG. (2006) The nob2 mouse, a null mutation in Cacna1f: anatomical and functional abnormalities in the outer retina and their consequences on ganglion cell visual responses. Vis Neurosci 23:11-24.

Chang Q, Gyftogianni E, Van De Graff SF, Hoefs S, Weidema FA, Bindels RJ and Hoenderop JG. (2004) Molecular determinants in TRPV5 channel assembly. J Biol Chem 279:54304-54311.

Cheever ML, Snyder JT, Gershburg S, Siderovski DP, Harden TK and Sondek J. (2008) Crystal structure of the multifunctional GB5-RGS9 complex. Nat Struct Mol Bio 15:155-162.

Chen CK, Eversole-Cire P, Zhang H, Mancino V, Chen YJ, He W, Wensel TG and Simon MI. (2003) Instability of GGL domain-containing RGS proteins in mice lacking the $G$ protein $\beta$-subunit Gß5. Proc Natl Acad Sci 100:6604-6609.

Chen FS, Shim H, Morhardt D, Dallman R, Krahn E, McWhinney L, Rao A, Gold SJ and Chen CK. (2009) Functional redundancy of R7 RGS proteins in ON-bipolar cell dendrites. Invest Ophthalmol Vis Sci 51:689-93.

Chen J, Kanai Y, Cowan NJ and Hirokawa N. (1992) Projection domains of MAP2 and tau determine spacing between microtubules in dendrites and axons. Nature 360:674-677.

Chevesich J, Kreuz AJ and Montell C. (1997) Requirement for the PDZ domain protein, INAD for localization of the TRP store-operated channel to a signaling complex. Neuron 18:95-105.

Chun MH, Grünert U, Martin PR and Wässle H. (1996) The synaptic complex of cones in the fovea and periphery of the macaque monkey retina. Vison Res 36: 3383-3395.

Cosens DJ and Manning A. (1969). Abnormal electroretinogram from a Drosophila mutant. Nature 224:285-287.

Conn PJ and Pin JP. (1997) Pharmacology and function of metabotropic glutamate receptors. Ann Rev Pharmacol Toxicol 37:205-237. 
Curcio CA, Sloan KR Jr, Packer O, Hendrickson AE and Kalina RE. (1987) Distribution of cones in human and monkey retina: individual variability and radial asymmetry. Science 236:579-582.

Curtis J and Finkbeiner S. (1999) Sending signals from the synapse to the nucleus: Possible roles for CaMK, RasOERK, and SAPK pathways in the regulation of synaptic plasticity and neuronal growth. J Neurosci Res 58, 88-95.

Deans MR, Volgyi B, Goodenough DA, Bloomfield SA and Paul DL. (2002) Connexin36 is essential for transmission of rod-mediated visual signals in the mammalian retina. Neuron 36:703712.

Demas J, Sagdullaev BT, Green E, Jaubert-Miazza L, McCall MA, Gregg RG, Wong RO and Guido W. (2006) Failure to maintain eye-specific segregation in nob, a mutant with abnormally patterned retinal activity. Neuron 50:247-259.

De Vries L, Zheng B, Fischer T, Elenko E and Farquhar MG. (2000) The regulator of G protein signaling family. Annu Rev Pharmacol Toxicol 40:235-271.

DeVries SH and Baylor DA (1993) Synaptic circuitry of the retina and olfactory bulb. Cell Suppl 72:139-149.

DeVries SH. (2000) Bipolar cells use kainate and AMPA receptors to filter visual information into separate channels. Neuron 28:847-856.

DeVries SH, Schwartz, EA. (1989) Modulation of an electrical synapse between solitary pairs of catfish horizontal cells by dopamine and second messengers. J Physiol 414:351-375.

DeVries SH, Schwartz EA. (1999) Kainate receptors mediate synaptic transmission between cones and 'Off' bipolar cells in a mammalian retina. Nature 397:157-160.

DeVries SH, Li W, Saszik S. (2006) Parallel processing in two transmitter microenvironments at the cone photoreceptor synapse. Neuron 50:735-748.

Dhingra A, Lyubarsky A, Jiang M, Pugh EN, Birnbaumer L, Sterling P and Vardi N. (2000) The light response of ON bipolar neurons requires Gao. J Neurosci 20:9053-9058.

Dhingra A, Jiang $M$, Wang $T$, Lyubarsky A, Savchenko A, Bar-Yehuda $T$, Stering $P$, Birnbaumer $L$ and Vardi N. (2002) Light response of retinal ON bipolar cells requires a specific splice variant of GaO. J Neurosci 22:4878-4884.

Dhingra A, Faurobert E, Dascal N, Sterling P and Vardi N. (2004) A retinal-specific regulator of gprotein signaling interacts with $\mathrm{G} \alpha \mathrm{O}$ and accelerates an expressed metabotropic glutamate receptor 6 cascade. J Neurosci 24:5684-5693.

Dizhoor AM. (2000) Regulation of cGMP synthesis in photoreceptors: role in signal transduction and congenital diseases of the retina. Cell Signal 12:711-719. 
Doering CJ, Rehak R, Bonfield S, Peloquin JB, Stell WK, Mema SC, Sauve Y and McRory JE. (2008) Modified Cav1.4 expression in the Cacnalfnob2 mouse due to alternative splicing of an ETn inserted in Exon 2. PLOS ONE 3:e2538.

Dowling JE and Boycott BB. (1966) Organization of the primate retina: Electron Microscopy. Proc R Soc London Ser B Biol Sci 166:80-111.

Dowling JE. (1970) Organization of vertebrate retinas. Invest Ophthalmol 9 655-680.

Dowling JE. (1987) The retina: An approachable part of the brain. Cambridge, MA: Harvard University Press.

Dryja TP, Berson EL, Rao VR and Oprian DD. (1993) Heterozygous missense mutation in the rhodopsin gene as a cause of congenital stationary night blindness. Nat Genet 4:280-283

Dryja TP, Hahn LB, Reboul T and Arnaud B. (1996) Missense mutation in the gene encoding the alpha subunit of rod transducin in the Nougaret form of congenital stationary night blindness. Nat Genet 13:358-360

Dryja TP, McGee TL, Berson EL, Fishman GA, Sandberg MA, Alexander KR, Derlacki DJ, Rajagopalan AS. (2005) Night blindness and abnormal cone electroretinogram ON responses in patients with mutations in the GRM6 gene encoding mGluR6. Proc Natl Acad Sci 102:4884-4889.

Duncan LM, Deeds J, Huner J, Shao J, Holmgren LM, Woolf EA, Tepper RI and Shyjan AW. (1998) Down-regulation of the novel gene melastatin correlates with potential for melanoma metastasis. Caner Research 58:1515-1520.

Duncan LM, Deeds J, Cronin FE, Donovan M, Sober AJ, Kauffman M and McCarthy JJ. (2001) Melastatin expression and prognosis in cutaneous malignant melanoma. J Clinical Oncology 19:568-576.

Erler I, Hirnet D, Wissenbach U, Flockerzi V and Niemeyer BA. (2004) Ca2+-selective transient receptor potential $\mathrm{V}$ channel architecture and function require a specific ankyrin repeat. J Biol Chem 279:34456-34463.

Ersfeld K, Wehland J, Plessmann U, Dodemont H, Gerke V and Weber K. (1993) Characterization of the tubulin-tyrosine ligase. J Cell Biol 120:725-732.

Field GD and Rieke G. (2002) Nonlinear signal transfer from mouse rods to bipolar cells and implications for visual sensitivity. Neuron 34:773-785.

Filipek S, Teller DC, Palczewski K, Stenkamp R. (2003) The crystallographic model of rhodopsin and its use in studies of other $\mathrm{G}$ protein-coupled receptors. Annu Rev Biophys Biomol Struct 32:375-397. 
Fishman GA, Stone EM, Sheffield VC, Gilbert LD and Kimura AE. (1992) Ocular findings associated with rhodopsin gene codon 17 and codon 182 transition mutations in dominant retinitis pigmentosa. Arch Ophthalmol 110:54-62.

Folsch H. (2008) Regulation of membrane trafficking in polarized epithelial cells. Curr Opin Cell Biol 20:208-213.

Freedman MS, Lucas RJ, Soni B, von Schantz M, Munoz M, David-Gray Z and Foster R. (1999) Regulation of mammalian circadian behavior by non-rod, non-cone ocular photoreceptors. Science 284:502-504.

Freymann DM, Keenan RJ, Stroud RM and Walter P. (1999) Functional changes in the structure of the SRP GTPase on binding GDP and Mg2_GDP. Nat Struct Biol 6:793-801.

Gal A, Orth U, Baehr W, Schwinger E and Rosenberg T. (1994) Heterozygous missense mutation in the rod CGMP phosphodiesterase beta-subunit gene in autosomal dominant stationary night blindness. Nat Genet 7:64-68

Garcia-Saez AJ, Chiantia S and Schwille P. (2007) Effect of line tension on the lateral organization of lipid membranes. J Biol Chem 282:33537-33544.

Gaudet R. (2008) TRP channels entering the structural era. J Physiol 586:3565-3575.

Gilbertson TA, Scobey R and Wilson M. (1991) Permeation of calcium ions through non-NMDA glutamate channels in retinal bipolar cells. Science 251:1613-1615.

Ghosh A and Greenberg ME. (1995) Calcium signaling in neurons: Molecular mechanisms and cellular consequences. Science 268:239-247.

Ghosh KK, Bujan S, Haverkamp S, Feigenspan A and Wässle H. (2004) J Comp Neurology 469:7082.

Goldsmith P, Backlund PS Jr, Rossiter K, Cater A, Milligan G, Unson CG and Spiegel A. (1988) Purification of heterotrimeric GTP-binding proteins from brain: identification of a novel form of Go. Biochemistry 27:7085-7090.

Gouras P. (1984) Color Vision. Prog Ret Res 3:227-261.

Gregg RG, Mukhopadhyaya S, Candille SI, Ball SL, Pardue MT, McCall MA, and Peachey NS. (2003) Identification of the gene and the mutation responsible for the mouse nob phenotype. Invest Opthalmol Vis Sci 44:378-384.

Gregg RG, Kanermans M, Klooster J, Lukasiewicz PD, Peachey NS, Vessey KA, and McCall MA. (2007) Nyctalopin expression in retinal bipolar cells restores visual function in a mouse model of complete X-linked congenital stationary night blindness. J Neurophysiol 98:302333. 
Groblewski T, Maigret B, Larguier R, Lombard C, Bonnafouc JC and Marie J. (1997) Mutation of Asn111 in the third transmembrane domain of the AT1A angiotensin II receptor induces its constitutive activation. J Biol Chem 272:1822-1826

Gu C, Jan YN and Jan LY. (2003) A conserved domain in axonal targeting of Kv1 (shaker) voltagegated potassium channels. Science 301:646-649.

Hack I, PeichI L and Brandstätter JH. (1999) An alternative pathway for rod signals in the rodent retina: rod photoreceptors, cone bipolar cells, and the localization of glutamate receptors. 96: $14130-14135$.

Haeseleer F, Imanishi Y, Maeda T, Possin DE, Maeda A, Lee A, Rieke F and Palczewski K. (2004) Essential role of $\mathrm{Ca2+-binding} \mathrm{protein} \mathrm{4,} \mathrm{a} \mathrm{Cav1.4} \mathrm{channel} \mathrm{regulator,} \mathrm{in} \mathrm{photoreceptor}$ synaptic function. Nat Neurosci 7:1079-1087.

Harteneck C. (2005) Function and Pharmacology of TRPM cation channels. NauynSchmiedeberg's Arch Pharmacol 371:307-314

Hattar S, Lucas RJ, Mrosovsky N, Thompson S, Douglas RH, Hankins MW, Lem J, Biel M, Hofmann F, Foster RG and Yau KW. (2003) Melanopsin and rod-cone photoreceptive systems account for all major accessory visual functions in mice. Nature 424:76-81.

Haverkamp S and Wässle H. (2000) Immunocytochemical analysis of the mouse retina. J Comp Neuro 424:1-23.

Haverkamp S, Grunert U and Wässle H. (2000) The cone pedicle, a complex synapse in the retina. Neuron 27:85-95.

Haynes LW, Kay A and Yau KW. (1986) Single cyclic GMP-activated channel activity in excised patches of rod outer segment membrane. Nature 321:66-70.

He W, Cowan CW and Wensel TG. (1998) RGS9, a GTPase accelerator for phototransduction. Neuron 20:95-102.

Hecht S, Schlaer S and Pirenne MH. (1942) Energy, quanta and vision. J Gen Physiol 25:819-840.

Heflin SJ, Nobles R, Health KL, Sagdullaev B, Vessey K, Guido W, Gregg RG and McCall MA. (2010) Receptive field organization of mouse retinal ganglion cells requires maintained input from On and Off pathways. J Neurophysiol (Submitted)

Heidelberger R, Sterling P and Matthews G. (2002) Roles of ATP in depletion and replenishment of the releasable pool of synaptic vesicles. J Neurophysiol 88:98-106.

Henry D, Burke S, Shishido E and Matthews G. (2003) Retinal bipolar neurons express the cyclic nucleotide-gated channel of cone photoreceptors. J Neurophysiol 89:754-761. 
Hildebrand A, Romaris M, Rasmussen L, Heinegard D, Twardzik D, Borders W and Ruoslathi $E$ (1994) Interactions of the small interstitial proteoglycans, decorin and fibromodulin with transforming growth factor b. Biochem J 302: 527-534.

Hirokawa, N. (1982) Cross-linker system between neurofilaments, microtubules, and membranous organelles in frog axons revealed by the quick-freeze, deep-etching method. $J$ Cell Biol 94:129-142.

Hirokawa $\mathrm{N}$ and Takemura R. (2005) Molecular motors and mechanisms of directional transport in neurons. Nat Rev Neurosci 6:201-214.

Hocking AM, Shinomura T and McQuillan DJ. (1998) Leucine-rich repeat glycoproteins of the extracellular matrix. Matrix Biol 17:1-19.

Hooks SB and Harden TK. (2004) Purification and in vitro functional analysis of R7 subfamily RGS proteins in complex with Gbeta5. Methods Enzymol 390:163-177.

Hope, Cl, Sharp DM, Hemara-Wahanui A, Sissingh JI, Lundon P, Mitchell EA, Maw MA and Clover GM. (2005) Clinical manifestations of a unique X-linked retinal disorder in a large New Zealand family with a novel mutation in CACNA1F, the gene responsible for CSNB2. Clin and Exp Ophthalmol 33:129-136.

Hunter JJ, Shao J, Smutko JS, Dussault BJ, Nagle DL, Woolf EA, Holmgren LM, Moore KJ and Shyjan AW. (1998) Chromosomal localization and genomic characterization of the mouse melastatin gene (Mlsn1). Genomics 54:116-123.

lozzo R. (1999) The biology of the small leucine-rich proteoglycans. J Biol Chem 274:1884318846.

Lasater EM and Dowling JE. (1982) Carp horizontal cells in culture respond selectively to Lglutamate and its agonists. Proc Natl Acad Sci USA 79:936-940.

Lee HC and Bernstein HD. (2001) The targeting pathway of excherichia coli presecretory and integral membrane proteins is specified by the hydrophobicity of the targeting signal. Proc Natl Acad Sci 13:3471-3476.

Lefkowitz RJ and Shenoy SK. (2005) Transduction of receptor signals by b-arrestins. Science 308:512-517

Lewis TL Jr, Mao T, Svodoba K and Arnold DB. (2009) Myosin-dependent targeting of transmembrane proteins to neuronal dendrites. Nat Neurosci 12:568-576.

Li Z, Sergouniotis PI, Michaelides M, Mackay DS, Wright GA, Devery S, Moore AT, Holder GE, Robson AG and Webster AR. (2009) Recessive mutations of the gene TRPM1 abrogate ON bipolar cell function and cause complete congenital stationary night blindness in humans. Am J Hum Genet 85:711-719. 
Li W, Chen S and DeVries SH. (2010) A fast rod photoreceptor signaling pathway in the mammalian retina. Nat Neurosci 13:414-416.

Liapakis G, Chan WC, Papadokostaki M and Javitch JA. (2004) Synergistic contributions of the functional groups of epinephrine to its affinity and efficacy at the b2 adrenergic receptor. Mol Pharmacol 65:1181-1190.

Liu $L$ and Simon SA. (1996) Capsaicin-induced currents with distinct desensitization and Calciumdependence in rat trigeminal ganglion cells. J Neurophysiol 75:1503-1514.

Lou Y and Denker BM. (1999) Interaction of heterotrimeric G protein Galphoao with Purkinje cell protein-2. Evidence for a novel nucleotide exchange factor. J Biol Chem 274:10685-10688.

Lucas RJ, Freedman MS, Munoz M, Garcia-Fernandez JM and Foster RG. (1999) Regulation of the mammalian pineal by non-rod, non-cone ocular photoreceptors. Science 284:505-507.

Luton F, Hexham MJ, Zhang M and Mostov KE. (2009) Identification of a cytoplasmic signal for apical transcytosis. Traffic 10:1128-1142.

Luttrell LM and Lefkowitz RJ. (2002) The role of b-arrestins in the termination and transduction of G-protein-coupled receptor signals. J Cell Sci 115:455-465

Ishii M, Morigiwa K, Takao M, Nakanishi S, Fukuda Y, Mimura O and Tsukamoto Y. (2009) Ectopic synaptic ribbons in dendrites of mouse retinal ON- anf OFF-bipolar cells. Cell Tissue Re 338:355-375.

Jacobi FK, Andreasson S, Langrova H, Meindl A, Zrenner E, Apfelstedt-Sylla E and Pusch CM. (2002) Phenotyic expression of the complete type of X-linked congenital stationary night blindness in patients with different mutations in the NYX gene. Graefes Arch Clin Exp Ophthalmol 240:822-828.

Jalkanen R, Mantyjarvi M, Tobias R, Alitalo T, and Bech-Hansen NT. (2004) X-linked cone-rod dystrophy (COD4) in a Finnish family is caused by mutation in CACNA1F. Am J Human Genet 75:A2593.

Jiang Y, Ruta V, Chen J, Lee A and MacKinnon R. (2003) The principle of gating charge movement in a voltage-dependent $K+$ channel. Nature 423:42-48.

Jonas P, Major G, Sakmann B. (1993) Quantal components of unitary EPSCs at the mossy fibre synapse on CA3 pyramidal cells of rat hippocampus. J Physiol 472:615-663.

Jung J, Lee SY, Hwang SW, Cho H, Shin J, Kang YS, Kim S and Oh U. (2002) Agonist recognition sites in the cytosolic tails of vanilloid receptor 1. J Biol Chem 277: 44448-44454.

Kabanarou SA, Holder GE, Fitzke FW, Bird AC and Webster AR. (2004) Congenital stationary night blindness and a "Schubert-Bornschein" type electrophysiology in a family with dominant inheritance. Br J Ophthalmol 88:1018-1022. 
Kaneko A. (1970) Physiological and morphological identification of horizontal, bipolar and amacrine cells in goldfish retina. J Physiol 207:623-633.

Kanaani J, el Husseini Ael D. Aguilera-Moreno A, Diacovo JM, Bredt DS and Baekkeskov S. (2002) A combination of three distinct trafficking signals mediates axonal targeting and presynatic clustering of GAD65. J Cell Biol 158:1229-1238.

Kanai $Y$ and Hirokawa N. (1995) Sorting mechanisms of tau and MAP2 in neurons: suppressed axonal transit of MAP2 and locally regulated microtubule binding. Neuron 14:421-432.

Keresztes G, Martemyanov KA, Krispel CM, Mutai H, Yoo PJ, Maison SF, Burns ME, Arshavsky VY, Heller S. (2004) Absence of the RGS9.Gbeta5 GTPase-activating complex in photoreceptors of the R9AP knockout mouse. J Biol Chem 279:1581-1584.

Kerschensteiner D, Morgan JL, Parker ED, Lewis RM and Wong RO. (2009) Neurotransmission selectively regulates synapse formation in parallel circuits in vivo. Nature 460:1016-1022.

Kim DS, Ross SE, Trimarchi JM, Aach J, Greenberg ME and Cepko CL. (2008) Identification of molecular markers of bipolar cells in the murine retina. J Comp Neurology 507:1795-1810.

Khawaja S, Gundersen GG and Bulinski JC. (1988) Enhanced stability of microtubules enriched in detyrosinated tubulin is not a direct function of detyrosination level. J Cell Biol 106:141-149.

Kobe B and Kajava AV. (2001) The leucine-rich repeat as a protein recognition motif. Curr Opin Struct Biol 11:725-732.

Kobilka BK, Kobilka TS, Daniel K, Regan JW, Caron MG and Lefkowitz RJ. (1988) Chimeric a2,b2adrenergic receptors: delineation of domains involved in effector coupling and ligand binding specificity. Science 240:1310-1316

Koike C, Obara T, Uriu Y, Numata T, Sanuki R, Miyata K, Koyasu T, Ueno S, Funabiki K, Tani A, Ueda $\mathrm{H}$, Kondo M, Mori Y, Masao T, Furukawa T. (2009) TrpM1 is a component of the retinal ON bipolar cell transduction channel in the mGluR6 cascade. Proc Natl Acad Sci

Kolb $H$. (1970) Organization of the outer plexiform layer of the primate retina: electron microscopy of Golgi-impregnated cells. Phil Trans R Soc B (Lond) 258:261-283.

Kolb H, Mariani A and Gallego A. (1980) A second type of horizontal cell in the monkey retina. J Comp Neurol 189:31-44.

Konishi $Y$ and Setou M. (2009) Tubulin tyrosination navigates the kinesin-1 motor domain to axons. Nat Neurosci 12:559-567.

Koplas PA, Rosenberg RL and Oxford GS. (1997) The role of calcium in the desensitization of capsaicin responses in rat dorsal root ganglion neurons. J Neurosci 17:3525-3537.

Koutalos $Y$ and Ebrey TG. (1986) Recent progress in vertebrate photoreception. Photochem. Photociol. 44:809-817. 
Kunishima N, Shimada Y, Tsuji Y, Sato T, Yamamoto M, Kumasaka T, Nakanishi S, Jingami H and Morikawa K. (2000) Structure basis of glutamate recognition by a dimeric metabotropic glutamate receptor. Nature 407:971-977.

Kwan KM, Fujimoto E, Grabher C, Mangum BD, Hardy ME, Campbell DS, Parant JM, Yost HJ, Kanki JP and Chien CB. (2007) The Tol2kit: A multisite gateway-based construction kid for Tol2 transposon transgenesis constructs. Dev Dynamics 236:3088-3099.

MacNeil MA and Masland RH. (1998) Extreme diversity among amacrine cells: implications for function. 20:971-982.

Maddox DM, Vessey KA, Yarbrough GL, Invergo BM, Cantrell DR, Inayat S, Balannik V, Hicks WL, Hawes NL, Byers S, Smith RS, Hurd R, Howell D, Gregg RG, Chang B, Naggert JK, Troy JB, Pinto LH, Nishina PM and McCall MA. (2008) Allelic variance between GRM6 mutants, $\mathrm{Grm6}^{\text {nob3 }}$ and $\mathrm{Grm} 6^{\text {nob4 }}$ results in differences in retinal ganglion cell visual responses. J Physiol 586:4409-4424.

Makino ER, Handy JW, Ki T and Arshavsky VY. (1999) The GTPase activating factor for transducin in rod photoreceptors is the complex between RGS9 and type $5 \mathrm{G}$ protein beta subunit. Proc Natl Acad Sci USA 96:1947-1952.

Magupalli VG, Schwarz K, Alpadi K, Natarajan S, Seigel GM and Schmitz F. (2008) Multiple RIBEYE-RIBEYE interactions create a dynamic scaffold for the formation of synaptic ribbons. J Neurosci 28:7954-7967.

Mangel SC. (1991) Analysis of the horizontal cell contribution to the receptive field surround of ganglion cells in the rabbit retina. J Physiol 442:211-234.

Mansergh F, Orton NC, Vessey JP, Lalonde MR, Stell WK, Tremblay F, Barnes S, Rancourt DE and Bech-Hansen NT. (2005) Mutation of the calcium channel gene Cacna1f disrupts calcium signaling, synaptic transmission and cellular organization in mouse retina. Hum Mol Genet 14:3035-3046.

Maruyama Y, Ogura T, Mio K, Kiyonaka S, Kato K, Mori Y and Sato C. (2007) Three-dimensional reconstruction using transmission electron microscopy reveals a swollen, bell-shaped structure of transient receptor potential melastatin-type 2 cation channel. J Biol Chem 282:36961-36970.

Martemyanov KA, Lishko PV, Calero N, Keresztes G, Sokolov M, Strissel KJ, Leskov IB, Hopp JA, Kolesnikov AV, Chen CK, Lem J, Heller S, Burns ME and Arshavsky VY. (2003) The DEP domain determines subcellular targeting of the GTPase activating protein RGS9 in vivo. J Neurosci 23:10175-10181.

Masu M, Iwakabe H, Tagawa Y, Miyoshi T, Yamashita J, Fukuda Y, Sasaki H, Hiroi K, Nakamura Y, Shigemoto R, Takada M, Nakamura K, Nakao K, Katsuki M and Nakanishi S (1995). Specific deficit of the ON response in visual transmission by targeted disruption of the mGluR6 gene. Cell 80: 757-765. 
Matlin AJ, Clark F and Smith CW. (2005) Understanding alternative splicing: towards a cellular code. Nat Rev Mol Cell Biol 6:386-398.

Matsushima N, Tachi N, Kuroki Y, Enkhbayar P, Osaki M, Kamiya M and Kretsinger RH. (2005) Structural analysis of leucine-rich repeat variants in proteins associated with human diseases. Cell Mol Life Sci 62:2771-2791.

McCall MA and Gregg RG. (2008) Comparisons of structural and functional abnormalities in mouse b-wave mutants. J Physiol 586:4385-4392.

McCall MA, Peachey NS and Gregg RG. (2008) Neurotransmission in the mouse retina. Eye, Retina, and Visual System of the mouse, ed. MIT Press: 175-188.

McEwan PA, Scott PG, Bishop PN and Bella J. (2006) Structural correlations in the family of small leucine-rich repeat proteins and proteoglycans. J Struct Bio 155:294-305.

Mehta D, Ahmmed GU, Paria BC, Holinstat M, Voyno-Yasenetskaya T, Tiruppathi C, Minsha RD and Malik AB. (2003) RhoA interaction with inositol 1,4,5-triphosphate receptor and transient receptor potential channel-1 regulates calcium entry. Role in signaling increased endothelial permeability. J Biol Chem 278:33492-33500.

Mills SL and Massey SC. (1994) Distribution and coverage of A- and B-type horizontal cells stained with Neurobiotin in the rabbit retina. Vis Neurosci 11:549-60.

Mio K, Ogura T, Kiyonaka S, Hiroaki Y, Tanimura Y, Fujiyoshi Y, Mori $Y$ and Sato C. (2007) The TRPC3 channel has a large internal chamber surrounded by signal sensing antennas. J Mol Biol 367:373-383.

Missotten L. (1965) The ultrastructure of the human retina (Brussels:Editions Arscia S.A.).

Miyake $Y$, Yagasaki K, Horiguchi M, Kawase $Y$ and Kanda T. (1986) Congenital stationary night blindness with negative electroretinogram: A new classification. Arch Ophthalmol 104:10131016.

Moiseenkova-Bell VY, Stanciu LA, Serysheva II, Tobe BJ and Wensel TG. (2008) Structure of TRPV1 channel revealed by electron cryomicroscopy. Proc Natl Acad Sci USA 105:7451-7455.

Moiseenkova-Bell VY and Wensel TG. (2009) Hot on the trail of TRP channel structure. J Gen Physiol 133:239-244.

Mojumder DK, Qian Y and Wensel TG. (2009) Two R7 regulator of G-protein signaling proteins shape retinal bipolar cell signaling. J Neurosci 29:7753:7765.

Montell C and Rubin GM. (1989) Molecular characterization of the Drosophila trp locus: a putative integral membrane protein required for phototransduction. Neuron 2: 1313-23.

Montell, C. (2005) The TRP superfamily of cation channels. Science STKE re3, 1-24. 
Morgans CW, Gaughwin P and Maleszka R. (2001) Expression of the alpha1F calcium channel subunit by photoreceptors in the rat retina. Mol Vis 7:202-209.

Morgans CW, Bayley PR, Oesch NW, Ren G, Akileswaran L, Taylor WR. (2005) Photoreceptor calcium channels: insight from night blindness. Vis Neurosci 22:561-568.

Morgans CW, Liu W, Wensel TG, Brown RL, Perez-Leon JA, Bearnot B and Duvoisin RM. (2007) GB5-RGS complexes co-localize with Grm6 in retinal ON-bipolar cells. European J Neuro 26:2899-2905.

Morgans CW, Puthussery T, Brown RL, Wensel TG, Jeffery B and Duvoisin RM. (2008) Gb5-RGS complexes interact with Grm6 in retinal on-bipolar cells. ARVO Poster 1285/A245.

Mosavi LK, Cammet TJ, Desrosiers DC and Peng ZY. (2004) The ankryin repeat as molecular architecture for protein recognition. Protein Science 13: 1435-1448.

Muto T, Tsuchiya D, Morikawa K and Jingami H. (2007) Structures of the extracellular regions of the group II/III metabotropic glutamate receptors. Proc Natl Acad Sci 104:3759-3764.

Nachman-Clewner M, St. Jules R and Townes-Anderson E. (1999) L-type calcium channels in the photoreceptor ribbon synapse: Localization and role in plasticity. J Comp Neuro 415:1-16.

Nakajima Y, Iwakabe H, Akazawa C, Nawa H, Shigemoto R, Mizuno N and Nakanishi S. (1993) Molecular characterization of a novel retinal metabotropic glutamate receptor mGluR6 with a high agonist selectivity for L-2-amino-4-phosphonobutyrate. J Biol Chem 268:1186811873.

Nakajima Y, Moriyama M, Hattori M, Minato N and Nakanishi S. (2009) Isolation of ON bipolar cell genes via hrGFP-coupled cell enrichment using the mGluR6 promoter. J Biochem 145:811-818.

Nakamura M, Ito S, Piao C-H, Terasaki H and Miyake Y. (2003) Retinal and optic disc atrophy associated with a CACNA1F mutation in a Japanese family. Arch of Ophthalmol 121:10281033.

Nakamura $M$, Sanuki R, Yasuma TR, Onishi A, Nishiguchi KM, Koike $C$, Kadowaki $M$, Kondo $M$, Miyake $Y$ and Furukawa T. (2010) TRPM1 mutations are associated with the complete form of congenital stationary night blindness. Mol Vis 16:425-437.

Nakata T and Hirokawa N. (2003) Microtubules provide directional cues for polarized axonal transport through interaction with kinesin motor head. J Cell Biol 162:1045-1055.

Nawy S and Copenhagen DR. (1987) Multiple classes of glutamate receptor on depolarizing bipolar cells in retina. Nature 325:56-58.

Nawy S and Jahr CE. (1990) Suppression by glutamate of cGMP-activated conductance in retinal bipolar cells. Nature 346,:269-271. 
Nawy S and Jahr CE. (1991) cGMP-gated conductance in retinal bipolar cells is suppressed by the photoreceptor transmitter. Neuron 7:677-683.

Nawy S. (1999) The metabotropic receptor mGluR6 may signal through $G(0)$, but not phosphodiesterase, in retinal bipolar cells. J Neurosci 19:2938-44.

Nawy S. (2000) Regulation of the on bipolar cell mGluR6 pathway by calcium. J Neurosci 20:4471-4479.

Nelson R. (1977) Cat cones have rod input: a comparison of the response properties of cones and horizontal cell bodies in the retina of the cat. J Comp Neurol 172:109-135.

Nomura A, Shigemoto R, Nakamura Y, Okamoto N, Mizuno N and Nakanishi S. (1994) Developmentally regulated postsynaptic localization of a metabotropic glutamate receptor in rat rod bipolar cells. Cell 77:361-369.

Nordquist DT, Kozak CA and Orr HT. (1988) CDNA cloning and characterization of three genes uniquely expressed in cerebellum by Purkinje neurons. J Neurosci 8:4780-4789.

Oancea E, Wolfe JT and Clapham DE. (2006) Functional TRPM7 channels accumulate at the plasma membrane in response to fluid flow. Circ Res 98:245-253.

Oancea E, Vriens J, Brauchi S, Jun J, Splawski I and Clapham DE. (2009) TRPM1 forms ion channels associated with melanin content in melanocytes. Sci Signal 2:ra21.

O'Conner E, Eisenhaber B, Dalley J, Wang T, Missen C, Bulleid N, Bishop PN and Trump D. (2005) Species specific membrane anchoring of nyctalopin, a small leucine-rich repeat protein. Human Mole Genetics 14:1877-1887.

Odell AF, Scott $J$ and Van Helden DF. (2005) Epidermal growth factor induces tyrosine phosphorylation, membrane insertion, and activation of transient receptor potential channel 4. J Biol Chem 280:37974-37987.

O'Hara PJ, Sheppard PO, Thogersen H, Venezia D, Haldeman BA, McGrane V, Houamed KM, Thomsen C, Gilbert TL and Mulvihill ER. (1993) The ligand-binding domain in metabotropic glutamate receptors is related to bacterial periplasmic binding proteins. Neuron 11:41-52.

Okamoto N, Hori S, Akazawa C, Hayashi Y, Shigemoto R, Mizuno N and Nakanishi S. (1994) Molecular characterization of a new metabotropic glutamate receptor mGluR7 coupled to inhibitory cyclic AMP signal transduction. J Biol Chem 269:1231-1236.

Pahlberg J, Okawa H, Birnbaumer L, Rieke F and Sampath AP. (2010) Cooperative control of sensitivity by two splice variants of $\mathrm{G} \alpha \mathrm{O}$ in retinal Rod ON Bipolar Cells. ARVO E-Abstract 4128/D624. 
Palczewski K, Kumasaka T, Hori T, Behnke CA, Motoshima H, Fox BA, Le Trong I, Teller DC, Okada T, Stenkamp RE, Yamamoto $M$ and Miyano M. (2000) Crystal structure of rhodopsin: a $G$ protein-coupled receptor. Science 289:739-745.

Palczewski K and Saari JC. (1997) Activation and inactivation steps in the visual transduction pathway. Curr Opin Neurobiol 7:500-504.

Pan F and Massey SC. (2007) Rod and cone input to horizontal cells in the rabbit retina. J Comp Neurology 500:815-831.

Panda S, Provencio I, Tu DC, Pires SS, Rollag MD, Castrucci AM, Pletcher MT, Sato TK, Wiltshire T, Andahazy M, Kay SA, Van Gelder RN, and Hogenesch JB. (2003) Melanopsin is required for non-image-forming photic responses in blind mice. Science 301: 525-527.

Pardue MT, McCall MA, LaVail, MM, Gregg RG and Peachey NS. (1998) A naturally-occuring mouse model of $X$-linked congenital stationary night blindness. Invest Opthalmol Vis Sci 39:2443-2449.

Park JH, Scheerer P, Hofmann KP, Choe HW and Ernst OP. (2008) Crystal structure of the ligandfree G-protein-coupled receptor opsin. Nature 454:183-187.

Parsons TD, and Sterling P. (2003) Synaptic ribbon: conveyor belt or safety belt? Neuron 37:379382.

Paternain AV, Rodriguez-Moreno A, Villarroel A and Lerma J. (1998) Activation and desensitization properties of native and recombinant kainate receptors. Neuropharmacology 37: 1249-1259.

Peachey NS and Ball SL. (2003) Electrophysiological anyalysis of visual function in mutant mice. Documenta Opthalmologica 107:13-36.

Perraud AL, Schmitz C and Scharenberg AM. (2003) TRPM2 Ca2+ permeable cation channels: from gene to biological function. Cell Calcium 33:519-531.

Perraud AL, Knowles HM and Schmitz C. (2004) Novel aspects of signaling and ion-homeostasis regulation in immunocytes. The TRPM ion channels and their potential role in modulating the immune response. Mol Immunol 41:657-673.

Pesch K, Zeitz C, Fries JE, Munscher S, Pusch CA, Kobler K, Berger W and Wissinger B. (2003) Isolation of the mouse nyctalopin gene Nyx and expression studies in mouse and rat retina. Invest Opthalmol Vis Sci 44:2260-2266.

Peterson JH, Woolhead CA and Bernstein HD. (2003) Basic amino acids in a distinct subset of signal peptides promote interaction with the signal recognition particle. J Biol Chem 14:46155-46162.

Phelps CB, Huang RJ,Wang RR and Gaudet R (2008). Structural analyses of the ankyrin repeat domain of TRPV6 and related TRPV ion channels. Biochemistry 47:2476-2484. 
Phelps CB, Procko E, Lishko PV, Wang RR and Gaudet R (2007). Insights into the roles of conserved and divergent residues in the ankyrin repeats of TRPV ion channels. Channels 1:148-151.

Pearring JN, Bojang P and Gregg RG. (2009) Nyctalopin expression in Grm6 mutant mice and membrane topology determine in yeast. Association for Research in Vision \& Opthalmology E-Abstract 1021.

Pillers DA, Fitzgerald KM, Duncan NM, Rash SM, White RA, Dwinnell SJ, Powell BR, Schnur RE, Ray PN, Cibis GW and Weleber RG. (1999) Duchenne/Becker muscular dystrophy: correlation of phenotype by electroretinography with sites of dystrophin mutations. Hum Genet 105:2-9.

Pinto LH, Vitaterna MH, Shimomura K, Siepka SM, Balannik V, Mcdermon EL, Omura C, Lumayag $S$, Invergo BM, Glawe B, Cantrell DR, Inayat S, Olvera MA, Vessey KA, McCall MA, Maddox D, Morgans CW, Young B, Pletcher MT, Mullins RF, Troy JB and Takahashi JS. (2007) Generation, identification and functional characterization of the nob4 mutation of Grm6 in the mouse. Visual Neurosci 24:111-123.

Piper AS, Yeats JC, Bevan S and Docherty RJ. (1999) A study of the voltage dependence of capsaicin-activated membrane currents in rat sensory neurons before and after acute desensitization. J Physiol 518:721-733.

Popescu DC, Ham AJL and Shieh BH. (2006) Scaffolding protein INAD regulates deactivation of vision by promoting phosphorylation of transient receptor potential by eye protein kinase $c$ in Drosophila. J Neurosci 26:8570-8577.

Pourcho RG and Goebel DJ. (1985) A combined Golgi and autoradiographic study of 3(H)glycineaccumulating amacrine cells in the cat retina. J. Comp. Neurol. 233: 473-480.

Pusch CM, Zeitz C, Brandau O, Pesch K, Achatz H, Feil S, Scharfe C, Maurer J, Jacobi FK, Pinckers A, Andreasson S, Hardcastle A, Wissinger B, Berger W and Meindl A. (2000) The complete form of $X$-linked congenital stationary night blindness is caused by mutations in a gene encoding a leucine-rich repeat protein. Nat Genet 26:324-327.

Rao VR, Cohen GB and Oprian DD. (1994) Rhodopsin mutation G90D and a molecular mechanism for congenital night blindness. Nature 367: 639-642.

Rao A, Dallman R, Henderson S and Chen CK. (2007) GB5 is required for normal light responses and morphology of retinal on-bipolar cells. J Neurosci 27:14199-14204.

Rao-Mirotznik R, Harkins AB, Buchsbaum G and Sterling P. (1995) Mammalian rod terminal: architecture of a binary synapse. Neuron 14:561-569.

Raviola E and Raviola G. (1982) Structure of the synaptic membranes in the inner plexiform layer of the retina: a freeze-fracture study in monkeys and rabbits. J Comp Neurol 209:233-248. 
Ribera JF, Ahmad S, Quick MW, Liman ER and Arnold DB. (2003) An evolutionarily conserved cileucine motif in Shal K+ channels mediates dendritic targeting. Nat Neurosci 6:243-250.

Rieke F and Schwartz EA. (1996) Asynchronous transmitter release: control of exocytosis and endocytosis at the salamander rod synapse. J Physiol (London) 493:1-8.

Robson JG and Frishman L. (1998) Dissecting the dark-adapted electroretinogram. Doc Ophthalmol 95:187-215.

Rosen LB, Ginty DD, Weber MJ and Greenberg ME. (1994) Membrane depolarization and calcium influx stimulate MEK and MAP kinase via activation of Ras. Neuron 12, 1207-1221.

Ruether K, Grosse J, Matthiessen E, Hoffmann K and Hartmann C. (2000) Abnormalities of the photoreceptor-bipolar cell synapse in a substrain of C57BL/10 mice. Invest Ophthalmol Vis Sci 41:4039-4047.

Saito T and Kaneko A. (1983) lonic mechanisms underlying the responses of off-center bipolar cells in the carp retina. Studies on responses evoked by light. J Gen Physiol 81:589-601.

Saito N, Okada Y, Noda Y, Kinoshita Y, Kondo S and Hirokawa N. (1997) KIFC2 is a novel neuronspecific C-terminal type kinesin superfamily motor for dendritic transport of multivesicular body-like organelles. Neuron 18, 425-438.

Sandmeyer LS, Breaux CB, Archer S and Grahn BH. (2007) Clinical and electroretinographic characteristics of congenital stationary night blindness in the Appaloosa and the association with the leopard complex. Veterinary Opthalmol 10:368-375.

Scholl HPN, Langrova H, Pusch CM, Wissinger B, Zrenner E and Apfelstedt-Sylla E. (2001) Slow and Fast rod ERG pathways in patients with $X$-linked complete stationary night blindness carrying mutations in the NYX gene. Invest Ophthalmol Vis Sci 42:2728-2736.

Schoneberg T, Liu J and Wess J. (1995) Plasma membrane localization and functional rescue of truncated forms of a G protein-coupled receptor. J Biol Chem 270:18000-18006.

Schmitz F, Konigstorfer A and Sudhof TC. (2001) RIBEYE, a component of synaptic ribbons: a protein's journey through evolution provides insight into synaptic ribbon function. Neuron 28:857-72.

Scott PG, McEwan PA, Dodd CA, Bergmann EM, Bishop PN and Bella J. (2004) Crystal structure of the dimeric protein core of decorin, the archetypal small leucine-rich repeat protein. Proc Natl Acad Sci 101:15633-15638.

Sedgwick SG and Smerdon SJ. (1999) The ankyrin repeat: a diversity of interactions on a common structural framework. Trends Biochem Sci 24:311-316.

Setou M, Nakagawa T, Seog DH and Hirokawa N. (2000) Kinesin superfamily motor protein KIF17 and $m$ Lin-10 in NMDA receptor-containing vesicle transport. Science 288:1796-1802. 
Shaner NC, Campbell RE, Steinbach PA, Giepmans BNG, Palmer AE and Tsien RY. (2004) Improved monomeric red, orange and yellow fluorescent proteins derived from Discosoma sp. Red fluorescent protein. Nature Biotech 22:1567-1572.

Sharma S, Ball SL and Peachey NS. (2005) Pharmacological studies of the mouse cone electroretinogram. Vis Neurosci 22: 631-636.

Sharpe LT, Stockman A and MacLeod Di. (1989) Rod flicker perception: scotopic duality, phase lags and destructive interference. Vision Res 29:1539-1559.

Sharpe LT, Fach CC and Stockman A. (1993) The spectral properties of the two rod pathways. Vision Res 33:2705-2720.

Shiells RA, Falk G and Naghshineh S. (1981) Action of glutamate and aspartate analogues on rod horizontal and bipolar cells. Nature 294:582-594.

Shiells RA and Falk G. (1994) Responses of rod bipolar cells isolated from dogfish retinal slices to concentration-jumps of glutamate. Vis Neurosci 11:1175-1183.

Shiells RA and Falk G. (2000) Activation of calcium-calmodulin kinase II induces desensitization by background light in dogfish retinal 'on' bipolar cells. J Physiol 528:327-338.

Sidman RL. (1961) Histogenesis of the mouse eye studied with thymidine [3H]. The structure of the eye. New York: Academic Press. 497-506.

Slaughter MM and Miller RF. (1981) 2-amino-4-phosphonobutyric acid: a new pharmacological tool for retina research. Science 211:182-185.

Smyth JT, Lemonnier L, Vazquez G, Bird GS and Putney Jr JW. (2006) Dissociation of regulated trafficking of TRPC3 channels to the plasma membrane from their activation by phospholipase C. J Biol Chem 281:11712-11720.

Snellman J and Nawy S. (2004) cGMP-dependent kinase regulates response sensitivity of the mouse on bipolar cell. J Neurosci 24:6621-6628.

Snellman J, Tejinder K, Shen Y and Nawy S. (2008) Regulation of ON bipolar cell activity. Progress Retinal Eye Res 1-12.

Sondek J and Siderovski DP. (2001) Gy-like (GGL) domains: new frontiers in G-protein signaling and $\beta$-propeller scaffolding. Biochem Parmacol 61:1329-1337.

Song AH, Wang D, Chen G, Li Y, Luo J, Duan S and Poo MM. (2009) A selective filter for cytoplasmic transport at the axon initial segment. Cell 136:1148-1160.

Song $\mathrm{JH}$, Song $\mathrm{H}$, Wensel TG, Sokolov $M$ and Martemyanov KA. (2007) Localization and differential interaction of R7 RGS proteins with their membrane anchors R7BP and R9AP in neurons of vertebrate retina. Mol Cell Neurosci 35:311-319. 
Soucy E, Wang Y, Nirenberg S, Nathans IJ and Meister M. (1998) A novel signaling pathway from rod photoreceptors to ganglion cells in mammalian retina. Neuron 21:481-493.

Sterling P and Demb JB. (2004) Retina. In: Shepherd GM, editor. The synaptic organization of the brain. New York: Oxford University Press. 217-269.

Stockman A, Sharpe LT, Ruther K and Nordby K. (1995) Two signals in the human rod visual system: a model based on electrophysiological data. Vis Neurosci 12:951-970.

Strathmann M, Wilkie TM and Simon MI (1990) Alternative splicing produces transcripts encoding two forms of the alpha subunit of GTP-binding protein Go. Proc Natl Acad Sci USA 87:6477-6481.

Strom TM, Nyakatura G, Apfelstedt-Sylla E, Hellebrand H, Lorenz B, Weber BH, Wutz K, Gutwillinger N, Ruther K, Drescher B, Sauer C, Zrenner E, Meitinger T, Rosenthal A and Meindl A. (1998) An L-type calcium-channel gene mutated in incomplete X-linked congenital stationary night blindness. Nat Genet 19:260-263.

Sullivan L, Makris GS, Dickinson P, Mulhall LE, Forrest S, Cotton RG and Loughnan MS. (1993) A new codon 15 rhodopsin gene mutation in autosomal dominant retinitis pigmentosa is associated with sectorial disease. Arch Ophthalmol 111:1512-1517.

Swaminath G, Xiang Y, Lee TW, Steenhuis J, Parnot C and Kobilka BK. (2004) Sequential binding of agonists to the $b 2$ adrenoceptor: kinetic evidence for intermediate conformational states. J Biol Chem 279:686-691.

Swaminath G, Deupi X, Lee TW, Zhu W, Thian FS, Kobilka TS and Kobilka B. (2005) Probing the b2 adrenoceptor binding site with catechol reveals differences in binding and activation by agonists and partial agonists. J Biol Chem 280: 22165-22171.

Tachibana M. (1985) Permeability changes induced by L-glutamate in solidary retinal horizontal cells isolated from Carassius auratus. J Physiol 358:153-167.

Tagawa Y, Sawai H, Ueda Y, Tauchi M and Nakanishi S. (1999) Immunohistochemical studies of metabotropic glutamate receptor subtype 6-deficient mice show no abnormality of retinal cell organization and ganglion cell maturation. J Neurosci 19:2568-2579.

Takada Y, Fariss RN, Tanikawa A, Zeng Y, Carper D, Bush R and Sieving PA. (2004) A retinal neuronal developmental wave of retinoschisin expression begins in ganglion cells during layer formation. Invest Ophthalmol Vis Sci 45:3302-3312.

Takahiko $M$ and Cepko C. (2003) Electroporation and RNA interference in the rodent retina in vivo and in vitro. Proc Natl Acad Sci 101:16-20.

Takahiko $M$ and Cepko C. (2007) Controlled expression of transgenes introduced by in vivo electroporation. Proc Natl Acad Sci 104:1027-1032. 
Takumida M, Ishibashi T, Hamamoto T, Hirakawa K and Anniko M. (2008) Expression of transient receptor potential channel melastin (TRPM) 1-8 and TRPA1 (ankyrin) in mouse inner ear. Acta Otolaryngol 8:1-11.

Tam BM and Moritz OL. (2009) The role of rhodopsin glycosylation in protein folding, trafficking and light-sensitive retinal degeneration. J Neurosci 29:15145-15154.

Terry RB, Archer S, Brooks S, Bernoco D and Bailey E. (2004) Assignment of the appaloosa coat colour gene (LP) to equine chromosome 1. Anim Genet 35: 134-137.

Tian L and Kammermeier PJ. (2006) G protein coupling profile of mFluR6 and expression of Ga proteins in retinal ON bipolar cells. Vis Neurosci 23:909-916.

tom Dieck S and Brandstätter JH (2006) Ribbon synapses of the retina. Cell Tissue Res 326:339346

Tsukamoto Y, Morigiaw K, Ueda M and Sterling P. (2001) Microcircuits for night vision in mouse retina. J Neurosci 21:8616-8623.

Valent QA, Kendall DA, High S, Kusters R, Oudega B, Luirink J. (1995) Early events in preprotein recognition in E.coli: interaction of SRP and trigger factor with nascent polypeptides. EMBO J 14:5494-5505.

Van Buren JJ, Bhat S, Rotello R, Pauza ME, Premkumar LS. (2005) Sensitization and translocation of TRPV1 by insulin and IGF-I. Mol Pain 1:17.

van Genderen MM, Bijveld MM, Claassen YB, Florijn RJ, Pearring JN, Meire FM, McCall MA, Riemslag FC, Gregg RG, Bergen AA and Kamermans M. (2009) Mutations in TRPM1 cause complete congenital stationary night blindness in human. Am J Hum Genet 85:730-736.

Vandaele S, Nordquist DT, Feddersen RM, Tretjakoff I, Peterson AC and Orr HT. (1991) Purkinje cell protein-2 regulatory regions and transgene expression in cerebellar compartments. Genes Dev 5:1136-1148.

Vardi N. (1998) Alpha subunit of Go localizes in the dendritic tips of ON bipolar cells. J Comp Neurol 395:43-52.

Vardi N, Duvoisin R, Wu G and Sterling P. (2000) Localization of mGluR6 to dendrites of ON bipolar cells in primate retina. J Comp Neurol 423: 402-412.

Vigh J and Witkovsky P. (1999) Sub-millimolar cobalt selectively inhibits the receptive field surround of retinal neurons. Vis Neurosci 16:159-168.

Voets T, Oqsianik G, Janssens A, Talavaera K and Nilius B. (2007) TRPM8 voltage sensor mutants reveal a mechanism for integrating thermal and chemical stimuli. Nat Chem Bio 3:174-182.

von Gersdorff H. (2001) Synaptic ribbons: versatile signal transducers. Neuron 29:7-10. 
von Gersdorff H, Vardi E, Matthews G and Sterling P. (1996) Evidence that vesicles on the synaptic ribbon of retinal bipolar neurons can be rapidly released. Neuron 16:1221-1227.

Wässle $\mathrm{H}$ and Boycott BB. (1991) Functional architecture of the mammalian retina. Physiol Rev 71:447-480.

Wässle H, Grunert U, Cook NJ and Molday RS. (1992) The cGMP-gated channel of rod outer segments is not localized in bipolar cells of the mammalian retina. Neurosci Lett 134:199202.

Watson AJ, Aragay AM, Slepak VZ and Simon MI. (1996) A novel form of the G protein beta subunit Gbeta5 is specifically expressed in the vertebrate retina. J Biol Chem 271:2815428160.

Werblin FS and Dowling JE. (1969) Organization of the retina of the mudpuppy. Necturus maculosus II. Intracellular recording. J Neurophysiol 32:339-355.

Wes PD, Xu XZ, Li HS, Chien F, Doberstein SK and Montell C. (1999) Termination of phototransduction requires binding of the NINAC myosin III and the PDZ protein INAD. Nat Neurosci 2:447-53.

West RW. (1978) Bipolar and horizontal cells of the gray squirrel retina: Golgi morphology and receptor connections. Vision Res 18:129-136.

Winnemoller M, Schmidt $G$ and Kresse $H$. (1991) Influence of decorin on Fibroblast adhesion to Fibronectin. Eur J Cell Biol 54:10-17.

Witte $\mathrm{H}$, Neukirchen D and BradkeF. (2008) Microtubule stabilization specifies initial neuronal polarization. J Cell Biol 180:619-632.

Wutz K, Sauer C, Zrenner E, Lorenz B, Alitalo T, Broghammer M, Hergersberg $M$, de la Chapelle A, Weber $B H$, Wissinger $B$, Meindl A and Pusch CM. (2002) Thirty distinct CACNA1F mutations in 33 families with incomplete type of XLCSNB and Cacna1f expression profiling in mouse retina. Eur J Hum Genet. 10:449-456.

Wycisk KA, Budde B, Feil S, Skosyrski S, Bussi F, Neidhardt J, Glaus E, Nurnberg P, Ruether K and Berger W. (2006a) Structural and functional abnormalities of retinal ribbon synapses due to CACNA2D4 mutation. Invest Ophthalmol Vis Sci 47:3523:3530.

Wycisk KA, Zeitz C, Feil S, Wittmer M, Forster U, Neidhardt J, Wissinger B, Zrenner E, Wilke R, Kohl $S$ and Berger W. (2006b) Mutation in the auxiliary calcium-channel subunit CACNA2D4 causes autosomal recessive cone dystrophy. Am J Hum Genet 79:973-977.

Xu L, Ball SL, Alexander KR and Peachey NS. (2003) Pharmacological analysis of the rat cone electroretinogram. Vis Neurosci 20:297-306.

Xu XZ, Li HS, Guggino WB and Montell C. (1997) Co-assembly of TRP and TRPL produces a distinct store-operated conductance. Cell 89:1155-1164. 
Xu XZ, Chien F, Butler A, Salkoff L and Montell C. (2000) TRPY, a Drosophila TRP-regulated subunit, forms a regulated cation channel with TRPL. Neuron 26:647-657.

Xu XZ, Moebius F, Gill DL and Montell C. (2001) Regulation of melastatin, a TRP-related protein, through interaction with a cytoplasmic isoform. Proc Natl Acad Sci 98:10692-10697.

Xu Y, Sulaiman P, Feddersen RM, Liu J, Smith RG and Vardi N. (2008) Retinal ON bipolar cells express a new PCP2 splice variant that accelerates the light response. J Neurosci 28:88738884.

Yamada, E. (1969) Some structural features of the fovea centralis in the human retina. Arch Ophthal 82:151-159.

Yamaguchi Y, Mann DM and Ruoslahti E. (1990) Negative regulation of transforming growth factor-beta by the proteoglycan decorin. Nature 346:281-284.

Yao X, Parnot C, Deupi X, Ratnala VR, Swaminath G, Farrens D and Kobilka B. (2006) Coupling ligand structure to specific conformational switches in the 32 -adrenoceptor. Nature Chem Biol 2:417-422.

Young RW (1985a) Cell differentiation in the retina of the mouse. Anat Rec 212: 199-205.

Young RW (1985b) Cell proliferation during postnatal development of the retina in the mouse. Brain Res 353: 229-239.

Zeitz C, Scherthan H, Freier S, Feil S, Suchow V, Schweiger S and Berger W. (2003) NYX (nyctalopin on chromosome $\mathrm{X}$ ), the gene mutated in congenital stationary night blindness, encodes a cell surface protein. Invest Opthalmol Vis Sci 44:4184-4191.

Zeitz C, van Genderen M, Neidhardt J, Luhmann UF, Hoeben F, Forster U, Wycisk K, Matyas G, Hoyng CG, Riemslag F, Meire F, Cremers FP and Berger W. (2005a) Mutations in GRM6 cause autosomal recessive congenital stationary night blindness with a distinctive scotopic $15-\mathrm{Hz}$ flicker electroretinogram. Invest Ophthalmol Vis Sci 46:4328-4335.

Zeitz C, Minotii R, Feil S, Matyas G, Cremers FP, Hoyng CB and Berger W. (2005b) Novel mutations in CACNA1F and NYX in Dutch families with X-linked congenital stationary night blindness. Mol Vis 11:179-183.

Zeitz C, Forster U, Neidhardt J, Feil S, Kalin S, Leifert D, Flor P, and Berger W. (2007) Night blindness-associated mutation in the ligand-binding, cysteine-rich, and intracellular domains of the metabotropic glutamate receptor 6 abolish protein trafficking. Human Mutation 28:771-780.

Zeitz C, Gross AK, Leifert D, Kloeckerner-Gruissem B, McAlear SD, Lemke J, Neidhardt J and Berger W. (2008) Identification and functional characterization of a novel rhodopsin mutation associated with autosomal dominant CSNB. Invest Ophthalmol Vis Sci 49:41054114. 
Zhang J, Jeffrey BG, Morgans CW, Burke NS, Haley TL, Duvoisin RM and Brown RL. (2010) RGS7 and -11 complexes accelerate the ON-bipolar cell light response. Invest Ophthalmol Vis Sci 51:1121-1129.

Zhang J and Wu SM. (2005a) Physiological properties of rod photoreceptor electrical coupling in the tiger salamander retina. J Physiol 564:849-862.

Zhang X, Huang J, McNaughton PA. (2005b) NGF rapidly increases membrane expression of TRPV1 heat-gated ion channels. EMBO J 24:4211-4223.

Zhang $\mathrm{N}$ and Townes-Anderson E. (2002) Regulation of structural plasticity by different channel types in rod and cone photoreceptors. J Neurosci 22:7065-7079.

Zhang Q, Xiao X, Li S, Jia X, Yang Z, Huang S, Caruso RC, Guan T, Sergeev Y, Guo X and Hejtmancik JF. (2007) Mutations in NYX of individuals with high myopia, but without night blindness. Mol Vis 13:330-336.

Zhang $\mathrm{X}$, Zhang $\mathrm{H}$ and Oberdick J. (2002) Conservation of the developmentally regulated dendritic localization of a Purkinje cell-specific mRNA that encodes a G-protein modulator: comparison of rodent and human Pcp2(L7) gene structure and expression. Brain Res Mol Brain Res 105:1-10. 


\section{APPENDICES}

\section{Appendix I: Transient expression of YFP-nyctalopin using in vivo Electroporation}

A major limitation in my ability to characterize nyctalopin functional interactions with TRPM1 is that there is no good in vitro model to study these structure function relationships. As described in Chapter IV, nyctalopin and TRPM1 were not properly localized in the plasma membrane when recombinantly expressed in HEK293T cells. Making modified nyctalopin or TRPM1 constructs and generating transgenic mouse lines is a costly and time consuming. To overcome this limitation I begun initial experiments to implement in vivo electroporation of plasmid constructs into the retina at PO of the mouse, as first described by Matsuda and Cepko (2004). A solution of DNA plasmid is directly injected into the subretinal space of neonatal mouse pups (PO), and electric pulses applied by using tweezer-type electrodes. The genes of interest are then transiently expressed in a variable number of cells in a pattern that is dictated mostly by the promoter being used.

Initial in vivo electroporation experiments were performed to determine if I could obtain sufficient expression of YFP-nyctalopin in DBCs of the $N y x^{n o b}$ mice. At post-natal day 30 (P30) an electroretinogram (ERG) were performed to assess visual function and at P45 the expression of YFP-nyctalopin in the retina was determined by immunohistochemical analysis using a GFP antibody.

I tested several promoters to determine which gave the most abundant expression of YFP-nyctalopin in the bipolar cells. The promoters I tested were either ubiquitously expressed, Human ubiquitin-3, or bipolar cell specific, GABAc $\rho 1$ and Calcium Binding Protein 5 (CABP5). 
Examples of the staining patterns are shown in Figure 37A-C. Human ubiquitin-3 promoter drove expression into all cell types, Figure 37A. Immunohistochemistry using a GFP antibody shows the region near the injection site has expression of YFP-nyctalopin, while the remaining retina, shown with the nuclear stain ToPro3, does not have staining for YFP-nyctalopin (Figure 37A). I observed GFP positive puncta in the OPL when YFP-nyctalopin was being driven by either of the two bipolar cell specific promoters, GABAc $\rho 1$ or CABP5 (Figure 37B,C). This OPL puncta represents the expression of YFP-nyctalopin on the dendrites of DBCs, which is consistent with YFP-nyctalopin expression in the transgenic mice. In addition, there is occasionally a cell that is completely stained (Figure $37 \mathrm{C}$ ). I do not know the cause of this but suspect it is because of the transient nature of the expression.

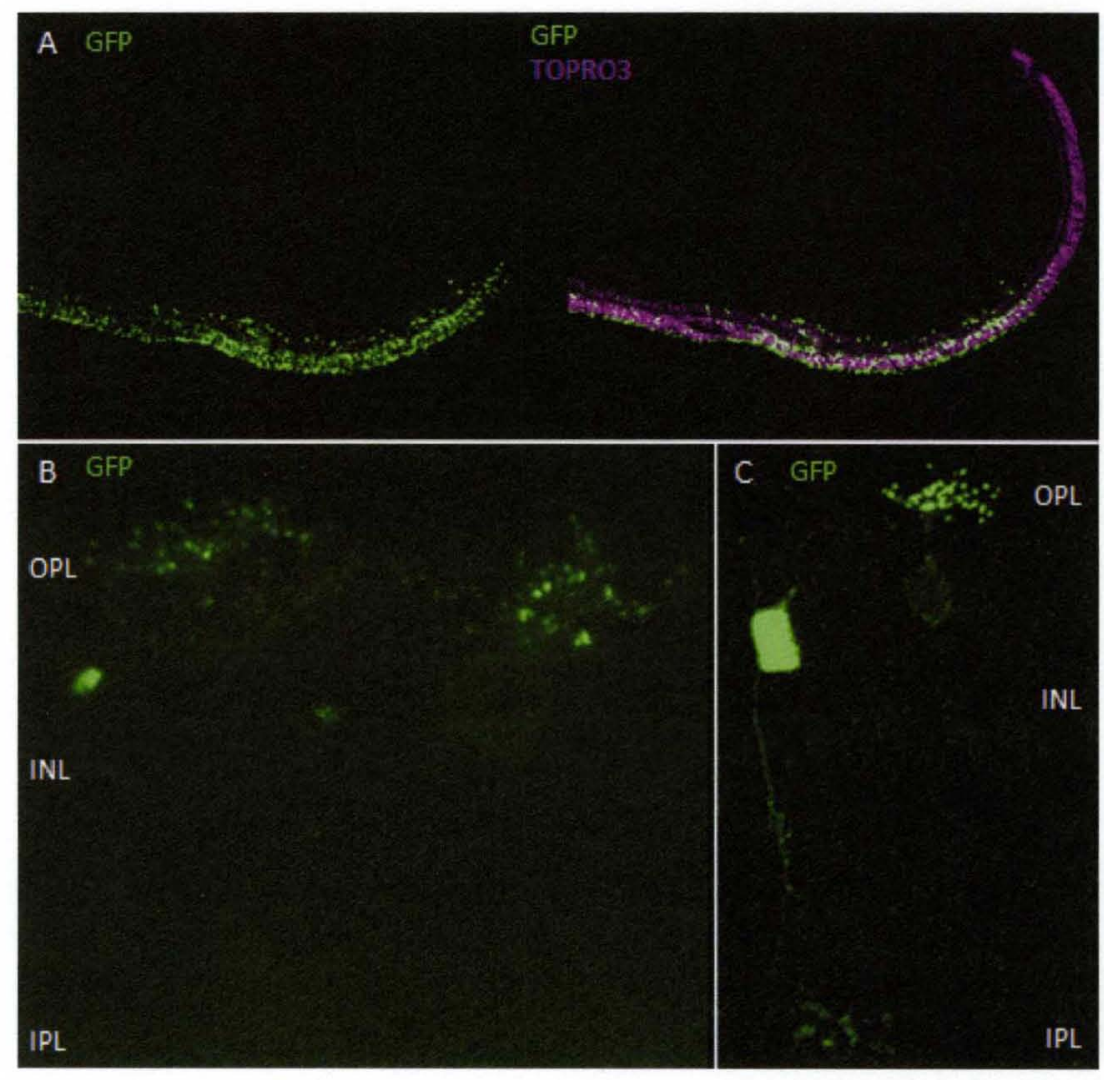

Figure 37. Immunohistochemical analysis of $\mathrm{Nyx}^{\text {nob }}$ retina at post-natal day 45 (P45) after in vivo electroporation of YFP-nyctalopin plasmids at PO. (A) Human-Ubiquitin3 promoter dives expression of YFP-nyctalopin (green) in all cell types. Double labeling with nucleic acid stain, TOPRO3, shows boundaries of YFP-nyctalopin expression in retina. (B) GABAcp1 promoter drives expression in of YFPnyctalopin to the dendritic tips of BCs. (C) CABP5 promoter also shows bipolar cells positively expressing GFP in their dendritic arbors. In addition, some cells do not properly express YFPnyctalopin. 
ERGs were performed at P45 to test whether transient expression of YFP-nyctalopin was sufficient to rescue the no b-wave ERG phenotype of $N y x^{n o b}$ mice. ERGs were normalized to the peak of the a-wave trough in the uninjected eye. Normalization accounts for the difference in amplitude of the a-wave in the injected verses uninjected eyes. A representative ERG from each injected plasmid is shown in Figure 38. Each graph plots the normalized ERG for the non-injected (pink, left) and the injected (blue, right) eyes as well as the difference trace (green, left-right) between the two eyes (Figure 38). In every mouse the uninjected control eye (pink) has a Nyx ${ }^{\text {nob }}$ ERG phenotype, a large a-wave and no b-wave (Figure 38A-D). In the YFP-nyctalopin injected eye, there is a shoulder present at the bottom of the a-wave that was initially thought to be a small b-wave (Figure 38A-C). This difference can be seen when subtracting the control ERG from the injected ERG (difference trace, green). However, when the negative control Hanks Balanced Salt Solution (HBSS) was electroporated the injected ERG showed the same shoulder present in YFP-nyctalopin injections (Figure 38D). The injection is causing a retinal damage that affects the ERG a-wave. This ERG difference is not dependent on YFP-nyctalopin expression.

Collectively, this data showed that transient expression of YFP-nyctalopin can target YFP-nyctalopin to DBC dendrites, but is not abundant enough to restore function to the DBCs. This technique could be used to transiently express nyctalopin or TRPM1 constructs and determine if they have the ability to properly locate to the DBC dendrites. 


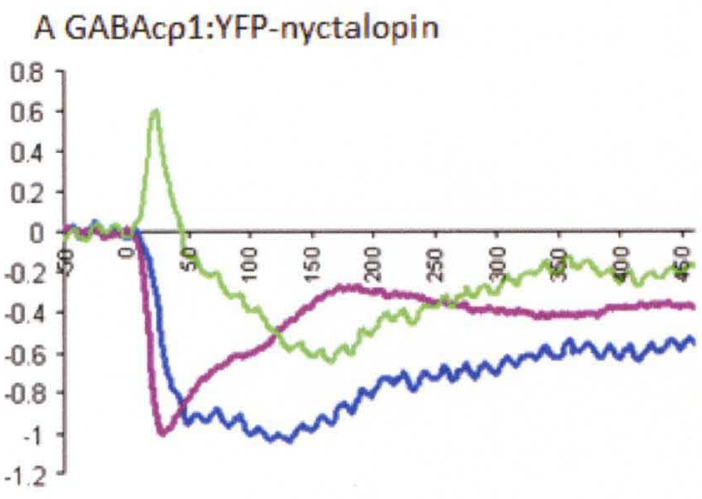

B Ubiquitin3:YFP-nyctalopin

C CABP5:YFP-nyctalopin
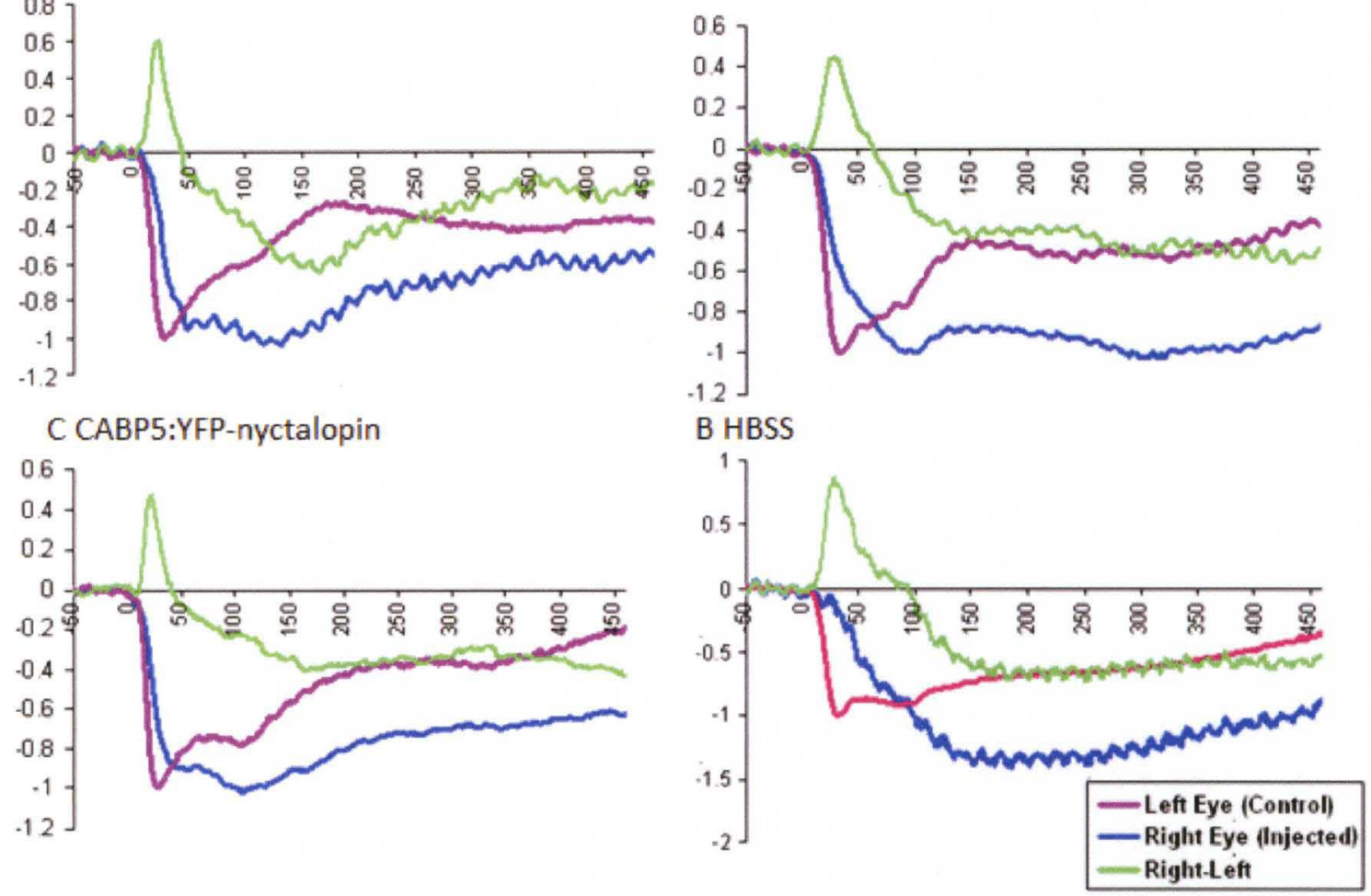

Figure 38. Normalized ERG from YFP-nyctalopin in vivo electroporated into $N y x^{\text {nob }}$ mice. ERG's were performed on the non-injected control eye (pink, left) and injected (blue, right) eye for each mouse. The control ERG was subtracted from the injected ERG and the difference plotted (green) to determine if the injection of YFP-nyctalopin rescued the ERG b-wave. Mice were injected with plasmids driving YFP-nyctalopin expression by a GABAcp1 (A), Ubiquitin3 (B) or CABP5 (C) promoters. Normalized ERGs of injected eye were indistinguishable from the HBSS injected control animals (D).

in vivo Electroporation: Newborn pups are anesthetized on ice, a cut made to open the eyelid and a small incision in the sclera near the cornea made with a 30-gauge needle. $0.5 \mu$ of PBS containing $2.5 \mu \mathrm{g}$ of plasmid DNA will be injected into the subretinal using a Hamilton syringe with a 33-gauge blunt-ended needle. After plasmid injection, tweezer-type electrodes $(7 \mathrm{~mm}$ diameter, Nepa Gene Co, Japan) briefly soaked in PBS were placed so that a current is passed across the head of the animal. Five $90 \mathrm{~V}$ square pulses of $50-\mathrm{ms}$ duration with 950 -ms intervals are applied by using a pulse generator (Nepa Gene Co, Japan). DNA was transfected only into the right eye and the left eye will be used as non-injected control. 


\section{Appendix II: Thrombin Digest of YFP-nyctalopin}

Nyctalopin is predicted to be highly glycosylated, so the exact molecular weight of YFPnyctalopin from retinal tissue remains unknown. In an attempt to reduce the size of YFPnyctalopin, the protein was screened for protease cleavage sites. Thrombin cleaves YFPnyctalopin at two sites, at amino acid 344 and 657 (Figure 39A). This digest is predicted to liberate the YFP tag from nyctalopin, which could be identified by immunoblotting with the GFP antibody. The predicted YFP would have a molecular mass of $36 \mathrm{kDa}$. Though this method will not identify full length YFP-nyctalopin it will help to determine if problems trying to detect YFPnyctalopin are due to glycosylation.

Membrane prepared fractions from $N y x^{n o b}$ and TgYFPNYX retinas were digested with restriction grade thrombin (Novagen) for 0,2 and 4 hours before analysis by western blot. Figure 39B shows that the GFP antibody did not detect a band in either the $N y x^{n o b}$ or $\operatorname{TgYFPNYX}^{\circ}$ fractions before or after thrombin digestion. RIBEYE also contains a thrombin cleavage site, which was used as an internal control for the thrombin digest. The membrane was stripped and re-probed with a RIBEYE antibody, which detects two bands from the retina $(90$ and $110 \mathrm{kDa}$, tom Dieck \& Brandstätter, 2005). Figure 39C shows that after a 2 or 4 hour digestion with thrombin the RIBEYE antibody detected two new bands at half the molecular weight of normal RIBEYE (60 and $50 \mathrm{kDa}$ ). These cleavage products were not present before digesting with thrombin. This data suggests that thrombin is digesting retina membrane proteins; however, a YFP fragment could not be detected.

To determine if the thrombin digest actually cleaved the predicted sites in YFPnyctalopin it was tested on the recombinant SYFP-NYX. HEK293T cell membrane fractions from transfections with mock or SYFP-NYX were subjected to the thrombin digest. Figure 390 shows the results from the western blot using a GFP antibody. A non-specific band at $65 \mathrm{kDa}$ is 
detected by the GFP antibody in every lane. The recombinant SYFP-NYX is detected only in SYFPNYX transfected cells; however, thrombin does not cleave the SYFP-NYX into a smaller $36 \mathrm{kDa}$ band as expected. The thrombin assay does not work on SYFP-NYX, which suggests it is also probably not working on YFP-nyctalopin from retina preparations.



VAGQDLSOVVFERSSDGLCVDDELYFTTSPGPSPEPVATTVSRYSSLLSKLLAPRAPVEEVANTTWELVSVSLEDFRSHAVMVCYKATFLFTSCVL 700

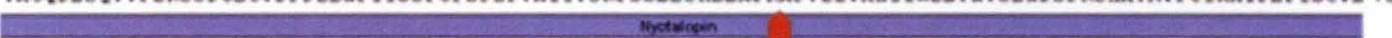

LSACTrVYGLORE

Hectiogen

$\mathrm{B}$

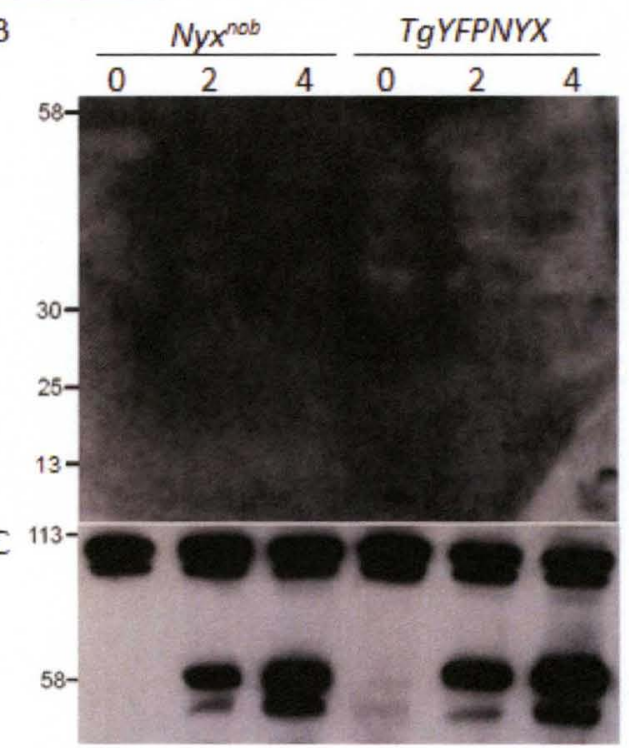

D

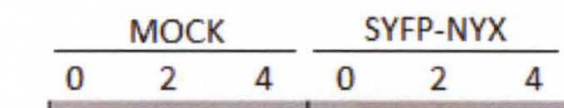

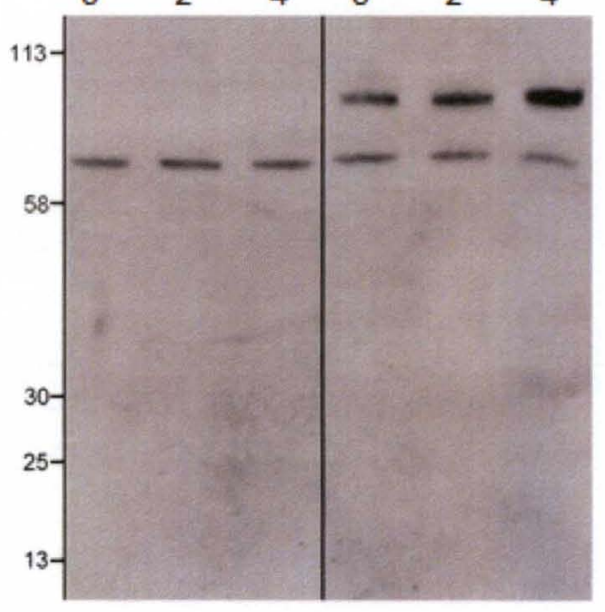

Figure 39: Thrombin digest of YFP-nyctalopin. (A) Schematic diagram of the predicted thrombin cleavage sites on YFP-nyctalopin. (B) Immunoblot with GFP antibody shows no cleavage of the SYFPNYX at 9OKDa from TgYFPNYX membrane preparation. (C) Immunoblot with RIBEYE antibody shows cleavage with thrombin after 2 and 4 hours digestion. (D) Thrombin digests on HEK293T cell lysate from mock or SYFP-NYX transfections. Recombinant SYFP-NYX is not cleaved by thrombin. A nonspecific band is present in every lane at 65KDa. 
Thrombin Digest Protocol: The membrane fraction from HEK293T cell and retinal lysates were used in the thrombin digest. $1 \times$ Cleavage Buffer (Novagen, Gibbstown, NJ) 30 ul Protein, $1 \mu$ of 1:25 dilution of Recombinant Grade Thrombin (Novagen, Gibbstown, NJ) up to final volume of $75 \mu \mathrm{l}$. The reactions were incubated at room temperature for 0,2 or 4 hours. $6 \mu$ l of $5 \times$ Sample Buffer was added to stop the reaction. Samples denatured at $85^{\circ} \mathrm{C}$ for 7 minutes before loading onto a $10 \%$ polyacrylamide gel. 


\section{JILLIAN PEARRING}

\section{CURRICULUM VITAE}

Department of Biochemistry \& Molecular Biology, University of Louisville 580 South Preston St, Louisville, KY 40202

e: jnpear01@louisville.edu / jillpearring@gmail.com

\section{Education}

2010. Doctor of Philosophy, Biochemistry \& Molecular Biology University of Louisville, Louisville, KY

2007. Master of Science, Biochemistry \& Molecular Biology University of Louisville, Louisville, $\mathrm{KY}$

2004. Bachelor of Arts, Biology Boston University, Boston, MA

2000. High School Diploma Manitou Springs High School, Manitou Springs, CO

\section{Dissertation}

Title. Nyctalopin is Required for Localization of the TRPM1 Channel to the Retinal Depolarizing Bipolar Cell Dendrites

Committee. Ronald Gregg (Chair), William Dean, Richard Feldhoff, Maureen McCall, Brian Wattenberg

\section{Publications}

Pearring JN, Bojang P, Shen Y, Nawy S, Gregg RG (In Preparation) A role for a small leucine rich repeat protein in targeting the TRPM1 channel to post-synaptic dendrites.

Van Genderen MM, Bijveld MM, Claassen YB, Florijn RJ, Pearring JN, Meire FM, McCall MA, Riemslag FC, Gregg RG, Bergen AA, Kamermans M (2009) Mutations in TRPM1 are a common cause of complete congenital stationary night blindness. American Journal of Human Genetics 85:730-6.

\section{Paper Presentations}

2009. Bojang P, Pearring JN, Gregg RG Nyctalopin interacts with transient receptor potential channels in yeast. Association for Research in Vision \& Ophthalmology EAbstract 5176.

2008. Pearring JN, Yarbrough GL, McCall MA, Gregg RG. In vivo electroporation of nyctalopin in nob mice. BMB Retreat, University of Louisville.

\section{Poster Presentations}

2010. Pearring JN, Gregg RG. Nyctalopin is required for proper localization of TRPM1 to depolarizing bipolar cell dendrites. FASEB Summer Conference on Retinal Neurobiology \& Visual Processing. Saxtons River, VT. 
2009. Pearring JN, Shen Y, Nawy S, Gregg RG. TRPM1 expression in depolarizing bipolar cells requires nyctalopin. Research! Louisville, University of Louisville Abstract GRD-59.

2009. Pearring JN, Bojang P, Gregg RG. Nyctalopin expression in Grm6 mutant mice and membrane topology determine in yeast. Association for Research in Vision \& Opthalmology E-Abstract 1021.

2005. Pearring JN, Deshler JO, Heflin SJ, Cook PB. Molecular characterization and localization of voltage-gated sodium channel in the tiger salamander retina. Association for Research in Vision \& Opthalmology E-Abstract 1713.

\section{Research Experience}

2005-2010. Graduate Research with Dr. Ronald Gregg, Chairman of Biochemistry \& Molecular Biology, University of Louisville

Molecular cloning, qRT-PCR, ERG, immunohistochemistry, cell culture, patch clamp, in vivo electroporation, and protein biochemistry techniques employed to study function of nyctalopin and TRPM1 channel in depolarizing bipolar cells.

2006. Experimental Tools in Model Systems of Translational Vision Research, Jackson Laboratories

Workshops attended: Basic tools for working with mice (Dr. Nishina \& Dr. Donnelly), Perfusions and retinal dissections (Dr. McCall \& Dr. Gregg), Electrophysiology (Dr. Pinto \& Dr. Chang), Retinal explants and electroporation (Dr. Dyer \& Dr. Donovan)

2004-2005. Research Technician to Dr. Paul Cook, Assistant Professor of Biology, Boston University Localized voltage-gated sodium channel mRNA in ambystoma tigrinum retina by in situ hybridization.

2002-2004. Undergraduate Research Project with Dr. Paul Cook, Assistant Professor of Biology, Boston University

Cloned the voltage-gated sodium channel from ambystoma tigrinum retinal cDNA library. Learned patch clamp techniques in ambystoma tigrinum retinal slice.

1998-1999. High School Research Project with Dr. Ralph Bertrand, Professor of Biology, Colorado College

Performed PCR and gel electrophoresis to study human population genetics.

Teaching Experience

2006. Teaching Assistant for Biochemistry (BIOC 545-645), University of Louisville

Fellowships, Grants and Awards

2010. Travel Grand ( $\$ 900)$ CGeMM, University of Louisville

2009. Travel Grant (\$750) National Eye Institute

2003-2004. Dean's List, Boston University

Professional Activities

2006-2008. Judge Kentucky State Science Fair Entries/Semi-Finals 